\title{
Computational Modelling and Electrical Stimulation for Epilepsy Surgery
}

Jurgen Hebbink

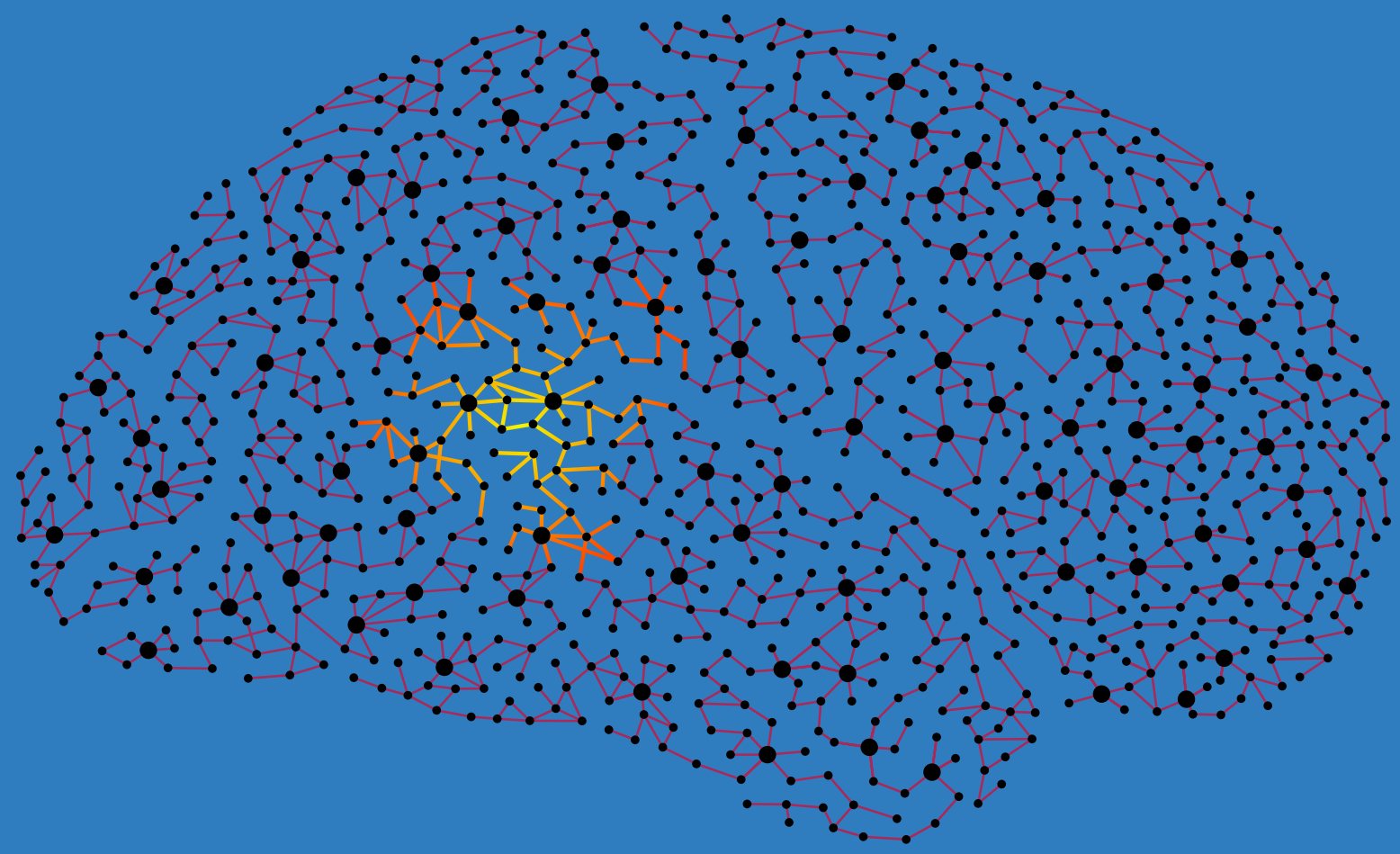




\title{
Computational Modelling and Electrical Stimulation for Epilepsy Surgery
}

\author{
Jurgen Hebbink
}


This work was financially supported by ZonMW/Dutch Epilepsy Foundation translational research grant 95104015 .

ISBN: 978-90-365-4832-8

DOI: $10.3990 / 1.9789036548328$

(C2019, Jurgen Hebbink, Zelhem, The Netherlands. All rights reserved. No parts of this thesis may be reproduced, stored in a retrieval system or transmitted in any form or by any means without permission of the author. Alle rechten voorbehouden. Niets uit deze uitgave mag worden vermenigvuldigd, in enige vorm of op enige wijze, zonder voorafgaande schriftelijke toestemming van de auteur. 


\section{COMPUTATIONAL MODELLING AND ELECTRICAL STIMULATION FOR EPILEPSY SURGERY}

\section{PROEFSCHRIFT}

ter verkrijging van

de graad van doctor aan de Universiteit Twente, op gezag van de rector magnificus,

prof.dr. T.T.M. Palstra,

volgens besluit van het College voor Promoties

in het openbaar te verdedigen

op woensdag 4 september 2019 om 14.45 uur

door

\section{Gerrit Jan Hebbink}

geboren op 4 september 1990

te Zelhem, Nederland 
Dit proefschrift is goedgekeurd door:
de promotor:
prof. dr. S.A. van Gils
de copromotoren:
dr. F.S.S. Leijten
dr. H.G.E. Meijer 


\section{Promotie Commissie}

Voorzitter

prof. dr. J.N. Kok

Promotor

prof. dr. S.A. van Gils (Universiteit Twente)

Copromotoren

dr. F.S.S. Leijten (Universitair Medisch Centrum Utrecht)

dr. H.G.E. Meijer (Universiteit Twente)

\section{Leden}

prof. dr. W. van Drongelen (The University of Chicago)

prof. dr. habil. T.R. Knösche (Max Planck Institute for Human Cognitive and Brain Sciences)

dr. ir. A. Hillebrand (VU Medisch Centrum)

prof. dr. ir. M.J.A.M. van Putten (Universiteit Twente)

prof. dr. N.V. Litvak (Universiteit Twente)

prof. dr. Yu.A. Kuznetsov (Universiteit Twente) 


\section{Contents}

1 Introduction 1

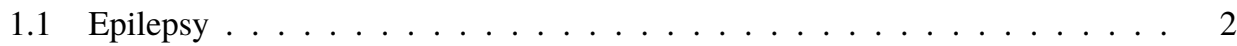

1.2 Epilepsy Surgery . . . . . . . . . . . . . . . . . . . . 3

1.3 Single Pulse Electrical Stimulation . . . . . . . . . . . . . . . . . . 3

1.4 Mathematical Models for Epilepsy . . . . . . . . . . . . . . . . . . . 4

1.5 Aim and Outline . . . . . . . . . . . . . . . . . 6

2 Phenomenological Network Models: Lessons for Epilepsy Surgery 9

2.1 Introduction . . . . . . . . . . . . . . . . . . . . 11

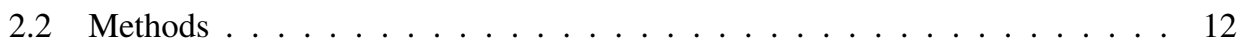

2.3 Results . . . . . . . . . . . . . . . . . . . . . . 14

2.4 Discussion . . . . . . . . . . . . . . . . . . . . . . 14

2.S1 Detailed Model Description . . . . . . . . . . . . . . . . . . 16

2.S2 Selected Seizure Networks . . . . . . . . . . . . . . . . . . 18

3 A Comparison of Evoked and Non-Evoked Functional Networks 35

3.1 Introduction . . . . . . . . . . . . . . . . 37

3.2 Materials and Methods . . . . . . . . . . . . . . . . . 38

3.3 Results . . . . . . . . . . . . . . . . . . . . 41

3.4 Discussion . . . . . . . . . . . . . . . . . . . 47

3.S1 Automatic Detection of ERs . . . . . . . . . . . . . . . 50

3.S2 Detailed Results of Other Patients . . . . . . . . . . . . . . . 52

3. S3 SPES \& Volume Conduction . . . . . . . . . . . . . . . . . . . . . 52

4 Pathological Responses to Single Pulse Electrical Stimuli in Epilepsy: The Role of Feedforward Inhibition

4.1 Introduction . . . . . . . . . . . . . . . . . . . 67

4.2 Materials and Methods . . . . . . . . . . . . . . . . . . 68

4.3 Results . . . . . . . . . . . . . . . . . . . . . . . 74

4.4 Discussion . . . . . . . . . . . . . . . . . . . . 81

4.S1 Model Details \& Additional Bifurcation Analysis . . . . . . . . . . . . . . . 84

4.S2 DR Onset Times in Other Patients . . . . . . . . . . . . . . . . . . 91 
5 On Analysis of Inputs Triggering Large Nonlinear Neural Responses 93

5.1 Introduction . . . . . . . . . . . . . . . . . . . 95

5.2 Material and Methods . . . . . . . . . . . . . . . . . . . 96

5.3 Results . . . . . . . . . . . . . . . . . . . . . . . 99

5.4 Conclusion \& Discussion . . . . . . . . . . . . . . . . . . . . 104

6 General Discussion $\quad 107$

6.1 Advancing Clinical SPES . . . . . . . . . . . . . . . . . . . . . . . . . 108

6.2 Network Models for Epilepsy Surgery . . . . . . . . . . . . . . . . . 111

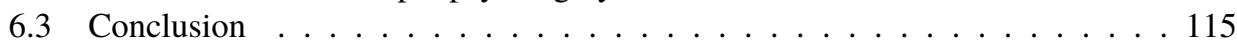

$\begin{array}{ll}\text { Appendices } & 117\end{array}$

List of Abbreviations . . . . . . . . . . . . . . . . . . . 118

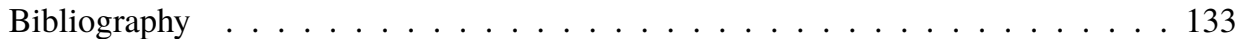

Summary . . . . . . . . . . . . . . . . . . . 134

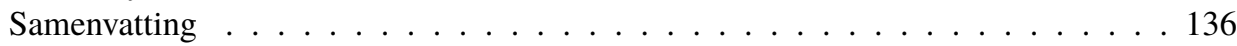

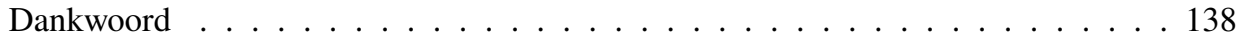



Chapter 1

Introduction 


\subsection{Epilepsy}

Epilepsy is a common neurological disease which actively affects around $0.4-1 \%$ of the word population [1,2]. Epilepsy is characterized by the occurrence of epileptic seizures, i.e. transient periods of abnormal excessive or synchronous neuronal activity [3]. Seizures occur only now and then, while most of the time epilepsy patients exhibit (relatively) normal neuronal activity. Conceptually, epilepsy is defined as an enduring predisposition to generate epileptic seizures [3]. A practical definition for diagnosing epilepsy requires any of the following three conditions to hold: (i) two unprovoked seizures occurring at least 24 hours apart from each other; (ii) the occurrence of an unprovoked seizure and a probability of further seizures similar to the general recurrence risk after two unprovoked seizures, occurring over the next 10 years or (iii) diagnosis of an epilepsy syndrome [4].

Epilepsy can have many different causes including structural and genetic defects [5]. Therefore, the way epilepsy is exposing in patients varies from sporadic, relatively mild seizures to frequent, disabling seizures. Based on their onset, seizures are classified as focal or generalized [6]. Focal seizures have a clear onset location that is limited to one hemisphere [7]. The onset pattern of focal seizures is consistent from one seizure to another. Propagation of focal seizures follows preferential pathways, which might include propagation to the other hemisphere. Generalized seizures start within distributed networks involving both hemispheres, but not necessarily the whole cortex [7]. Although individual generalized seizures may seem to have a localized onset, this onset location is not consistent between seizures. Patients can have different types of seizure including a mixture of focal and generalized seizures [5].

Multiple methods exist to treat epilepsy, e.g. antiepileptic drugs, epilepsy surgery, and electrical stimulation $[8,9]$. Treatment with antiepileptic drugs is usually the first choice and is successful in roughly $70 \%$ of the patients [10]. Different types of epilepsy require different antiepileptic drugs and therefore correct classification of the seizure type is of importance [11]. In around 50\% of the patients the first prescribed antiepileptic drugs is effective [12]. The second and third prescribed antiepileptic drug each control seizures in another $10 \%$ of the patients, while further drugs do not add much to this [12]. According to the current guidelines, a patient is drug-resistant when (s)he is not responding to two adequate trials of appropriately chosen antiepileptic drug schedules [13]. Curiously, the ratio of drug-resistant patients has remained constant for decades despite the increasing number of available antiepileptic drugs $[10,14]$, suggesting that pharmacological treatment alone is not sufficient to suppress all factors leading to seizures.

If treatment with antiepileptic drugs fails, epilepsy surgery might be an option. Epilepsy surgery is described in more detail in the next section. Drug-resistant patients who are not eligible for surgery may be treated with electrical stimulation using an implanted stimulation device. Three different methods exist, i.e. vagus nerve stimulation, deep-brain stimulation, and cortical electrical stimulation [15]. The most common method is stimulation of the vagus nerve[16]. As the vagus nerve has many projections within the central nervous system, it is thought that by stimulation of this nerve the entire brain can be affected, however the precise action mechanism of vagus nerve stimulation is unknown [16]. Long-term seizure freedom is obtained in $5-10 \%$ of the patients only [16], while the median reduction of the seizure rate is around 28\% [17]. Deep-brain stimulation and cortical electrical stimulation are both relatively new methods and target respectively deep-brain structures, e.g. the anterior nucleus 
of the thalamus and neocortical epileptic foci. A median decrease in seizure rate of $40 \%$ for deep brain stimulation in the anterior thalamus and $38 \%$ for cortical electrical stimulation has been reported [17].

\subsection{Epilepsy Surgery}

Epilepsy surgery may provide a cure for patients with focal seizures. The success rate of epilepsy surgery is $40-60 \%$ and is influenced by multiple factors, e.g. location of the epileptic focus and pathology $[18,19]$. The aim of epilepsy surgery is to remove the epileptogenic zone (EZ), defined as the smallest area of cortex whose removal yields seizure freedom [20, 21]. Surgery is only considered an option when the hypothesized EZ is not part of eloquent cortex, e.g. brain tissue involved in speech or motor function. The epileptogenic zone is mostly a theoretical notion, as in practice it can only be concluded that the EZ is completely removed if a patient is seizure-free after surgery. In such a case, the EZ is contained in the resected area (RA).

In clinical practice various methods involving different imaging modalities are used to approximate the EZ, e.g. magnetic resonance imaging (MRI), electroencephalography (EEG), and magnetoencephalography (MEG). Using MRI scans focal lesions can be visualized. Some focal lesions can facilitate epilepsy [22], although in some cases not the lesions itself but the tissue surrounding it is responsible for seizure generation [20]. With EEG and MEG one records neuronal activity based on the electrical and magnetic fields generated by this activity, respectively [23]. Normal EEG, measured with electrodes on the scalp, is widely applied and one of the standard methods in presurgical evaluation for epilepsy surgery. MEG is much more expensive than EEG, but has the advantage that the magnetic field is much less disturbed by the skull and skin compared to the electric field and therefore reconstruction of the neuronal sources from MEG signals is more straightforward than from EEG signals [23].

In complex cases non-invasive methods are not sufficiently accurate to approximate the EZ. In those cases intracranial EEG (iEEG), recorded with either depth electrodes (stereotactic EEG, SEEG) or electrode grids placed directly on the cortex (electrocorticography, ECoG), are needed, as they offer greater precision $[24,25]$. The seizure onset zone (SOZ) determined from unprovoked seizures recorded with ECoG remains the gold standard for localizing the EZ [25], although interictal recordings also contain biomarkers for epileptogenicity such as spikes [21] and especially high frequency oscillations (HFOs) [26, 27].

\subsection{Single Pulse Electrical Stimulation}

Single pulse electrical stimulation (SPES) is an alternative method to probe epileptogenicity during iEEG recordings. SPES does not rely on spontaneous interictal or ictal events, as it evokes brain responses using brief electrical pulses. During SPES, pairs of adjacent electrodes receive a stimulation for a typical duration of $1 \mathrm{~ms}$ and a strength of 4-8 mA, see Figure 1.1. Each electrode pair usually receives ten stimulation trials at a frequency of $0.2 \mathrm{~Hz}$. The stimulation evokes responses called cortico-cortical evoked responses (CCEP), which are recorded at the non-stimulated electrodes. Responses cannot be recorded at the stimulated electrodes, as these become saturated upon the stimulation for some time. Commonly, two types of responses are distinguished, i.e. early responses (ERs) and delayed responses (DRs) 
[28]. Besides these responses also 'stable' and 'repetitive' response are described in literature $[29,30]$.

ERs have an onset starting within $100 \mathrm{~ms}$ after the stimulation. They have a consistent timing and shape across stimulation trials as shown in the example in Figure 1.1B. The waveform of ERs usually consists of two negative deflections, called N1 and N2, although one of these components might be absent [31]. The N1 is a sharp wave reaching its minimum 10-50 ms after stimulation, while the $\mathrm{N} 2$ is a broad slow wave having its maximum amplitude somewhere between 100-200 ms. ERs are normal, non-epileptic responses representing direct cortical propagation [32-34]. Multiple studies have therefore used SPES to investigate connectivity in different brain regions such as the language area and motor cortex; an overview is given in [34]. When SPES is performed systematically over the whole electrode grid, the resulting ERs can be used to construct directed brain networks. These networks partly explain seizure propagation [35, 36]. Moreover, although ERs are non-epileptic responses, network measures calculated from ER networks exhibit differences between nodes inside and outside the SOZ and RA [37, 38].

DRs occur between 100 and $1000 \mathrm{~ms}$ after stimulation [28]. A typical feature of DRs is their stochastic occurrence, i.e. they arise only in a subset of the stimulation trials of the same electrode pair, see Figure 1.1B. The timing and shape of DRs varies across trials. DRs are a biomarker for epileptogenic cortex and have been observed in different brain regions in both adults and children [28, 30, 39, 40]. Time-frequency decomposition of SPES responses allows the assessment of DR activity in three frequency bands, i.e. spike $(10-80 \mathrm{~Hz})$, ripple $(80-250 \mathrm{~Hz})$, and fast-ripple $(250-500 \mathrm{~Hz})$ bands. DR activity in the spike and ripple band shows a high sensitivity toward the SOZ, while fast-ripple activity, occurring less frequently, is very specific for epileptogenic cortex [40].

Stable responses are small spikes or sharp waves emerging mostly on the rising slope of the N2 component of an ER [30]. Like ERs, stable responses have a consistent appearance and timing. The occurrence of stable responses exhibits no clear relationship with the SOZ [30]. Repetitive response emerge exclusively in the frontal lobe [29]. Their waveform consist of at least one repetition of the preceding ER component. Like DRs, the occurrence of stable responses is stochastic, however their latency is consistent [29]. Removal of stimulation sites evoking repetitive responses is associated with a good surgical outcome [29, 30].

\subsection{Mathematical Models for Epilepsy}

In the last half century mathematical models have been used to gain more insight in epilepsy. Computational modelling offers a complementary approach for understanding neuronal dynamics in relation to experimental data recorded from humans, animals or brain tissue cultures. In a computational modelling framework experimental data of various sources can be integrated and the role of variables that cannot be observed experimentally can be investigated [41]. Moreover, mathematical analysis of these models may give insight in the complex non-linear dynamics present in the brain. In epilepsy, models can be used to study both epileptic and non-epileptic brain activity, and to provide mechanistic explanations for seizure generation and propagation.

Computational models for neuronal activity exists for different scales ranging from single neuron to the whole brain $[41,42]$. Detailed models describe the activity of single neurons 
A

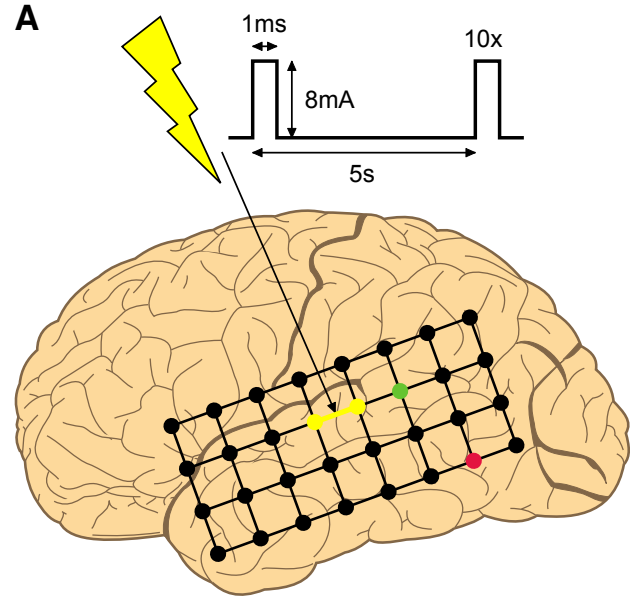

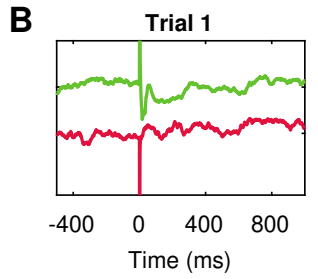
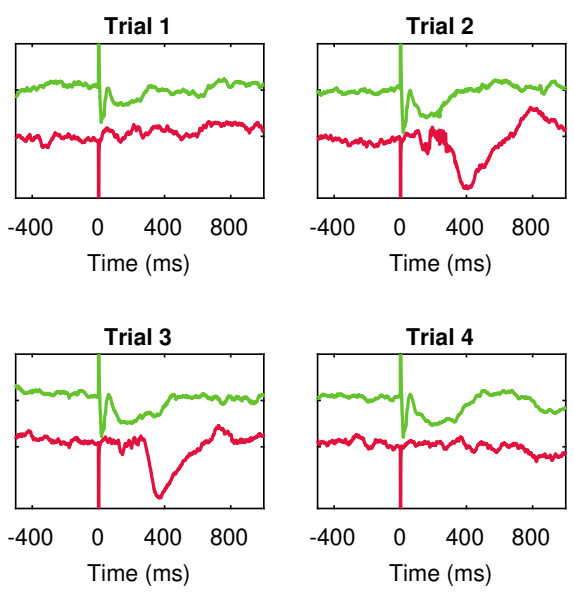

Figure 1.1: Overview of single pulse electrical stimulation (SPES). (A) Two adjacent electrodes from the intracranial electrode grid receive a short electrical stimulation, typically this is repeated ten times. (B) Examples of recorded responses at two non-stimulated electrodes for four stimulation trials of the same electrode pair. The green electrode shows an early responses on all stimulation trials, while the red electrode shows a delayed responses on trials 2 and 3 and no response at trials 1 and 4 .

and model processes on cellular level. Most of these models build on the traditional model by Hodgkin and Huxley [42, 43]. Network activity can be studied using simulations of networks consisting of up to several thousands of neurons. Compared to realistic neuronal networks this number is still small, e.g. the number of neurons in a cortical column is in the order of $10^{4}-10^{8}$ [44], but simulating larger models is computationally too expensive. The activity of larger groups of neurons can be studied using lumped models, e.g. neural mass models (NMMs) and neural field models. NMMs consider the average neuronal activity of a population rather than that of single neurons. The scale on which they describe neuronal dynamics, i.e. a few square millimeters up to a couple square centimeters of cortex, is comparable to the scale on which (intracranial) EEG records neuronal activity, allowing an easy (quantitative) comparison with clinical data. Neural field models take a continuum limit of neurons in space to describe the dynamics of a spatially extended cortical sheet [44]. They are used to study spatial-temporal patterns and traveling waves $[45,46]$. Further, also phenomenological models exist, e.g. the Epileptor [47] and bistable oscillators [48, 49]. These models do not simulate biophysical processes but offer mostly a simple mathematical description capturing some basic properties of neuronal systems to explain phenomena observed in data.

In this thesis we mainly focus on NMMs. The development of these models dates back to the early seventies to investigate the $\alpha$-rhythm in the thalamus [50]. Later on, the Jansen-Rit NMM was proposed as a physiological model for cortical dynamics [51, 52]. This NMM is still used nowadays and forms the basis for more detailed cortical NMMs [53-56]. It models activity of three different neuronal populations, i.e. pyramidal cells and local inhibitory and excitatory cells. The Jansen-Rit neural mass can simulate normal ongoing EEG activity as well as the $\alpha$-rhythm and spike-wave activity. An extension of the Jansen-Rit NMM is the Wendling model [53], which distinguishes fast and slow inhibition. The dynamic repertoire of the Wendling neural mass includes that of the Jansen-Rit neural mass and also comprises of 
low amplitude fast oscillatory activity and poly spike-wave activity [57]. The Wendling model can reproduce seizure dynamics observed in clinical practice by slowly varying parameters [53]. Besides various types of normal and epileptic activity, NMMs also allow to model various types of event-related responses [51, 56, 58, 59].

Where a single neural mass describes the activity of a small piece of cortex, multiple neural masses can be coupled to study the activity of larger brain networks. Coupled neural masses can generate an even larger variety of neuronal activity [60,61]. Networks of coupled neural masses can simulate seizure propagation $[62,63]$ and also propagation of activity due to transient perturbations $[59,64]$. An interesting, recent development is the use of coupled models for neuronal activity, being neural masses or more generally non-linear oscillators, to predict the effect of epilepsy surgery [65-67].

\subsection{Aim and Outline}

Accurate delineation of the epileptogenic zone remains one of the challenges in epilepsy surgery. Although epilepsy surgery nowadays is applied in more complex cases than before, the overall success rate is steady [68], which means there is still room for improvement. Epilepsy surgery mainly focuses on removing pathological cortex in the anatomic sense. Recent developments suggest that epilepsy is also a network disease $[69,70]$, as brain connectivity in epilepsy patients differs from healthy controls [71-73]. Computational models offer a framework to study both the effects of intrinsic epileptogenicity and network interaction. SPES may offer an opportunity to infer both patient-specific connectivity and information about local excitability. Therefore, in this thesis we investigate the added value of combining computational network models and SPES for epilepsy surgery.

We start in chapter 2 by investigating the role of both intrinsic epileptogenicity and network interaction in epilepsy surgery. We study the effect of surgery on the seizure rate in computational network models consisting of four nodes. One of the populations can be made hyperexcitable, modelling a pathological region of cortex. We investigate if removing this hyperexcitable node reduces the seizure rate and we search for the best node to remove.

Early responses evoked during SPES offer a direct way to infer connectivity. A question that arises is to what extent these SPES networks are similar to other, more common methods to infer brain connectivity. This question is investigated in chapter 3 , where we compare networks derived using SPES with two methods inferring connectivity from ongoing neuronal activity, i.e. correlation and Granger causality.

Next, in chapter 4, we focus on DRs evoked during SPES. While the clinical value of DRs is well established, the mechanism underlying DRs and especially their stochastic occurrence remained up till now elusive. Using a data-driven approach we study both early and delayed responses. The neural mass model we propose is able to explain some of the waveforms of the responses and the typical stochastic appearance of DRs.

In chapter 5 we study the mechanism proposed to model DRs in chapter 4 mathematically in more detail. This mechanism is an example of a large non-linear response to a short transient input appearing abruptly while increasing the stimulation strength in an excitable system. We use slow-fast analysis to study the transition from small, more or less linear responses to large non-linear ones.

Finally, the main findings of this thesis will be summarized and discussed in chapter 6 , 
leading to directions for future research. 



\section{Chapter 2}

\section{Phenomenological Network Models: Lessons for Epilepsy Surgery}

Jurgen Hebbink, Hil Meijer, Geertjan Huiskamp, Stephan van Gils, Frans Leijten 


\section{Abstract}

The current opinion in epilepsy surgery is that successful surgery is about removing pathological cortex in the anatomic sense. This contrasts with recent developments in epilepsy research where epilepsy is seen as a network disease. Computational models offer a framework to investigate the influence of networks, as well as local tissue properties, and to explore alternative resection strategies. Here we study, using such a model, the influence of connections on seizures and how this might change our traditional views of epilepsy surgery.

We use a simple network model consisting of four interconnected neuronal populations. One of these populations can be made hyperexcitable, modeling a pathological region of cortex. Using model simulations the effect of surgery on the seizure rate is studied.

We find that removal of the hyperexcitable population is, in most cases, not the best approach to reduce the seizure rate. Removal of normal populations located at a crucial spot in the network, the 'driver', is typically more effective in reducing seizure rate.

This work strengthens the idea that network structure and connections may be more important than localizing the pathological node. This can explain why lesionectomy may not always be sufficient. 


\subsection{Introduction}

Epilepsy surgery has provided a cure for patients with focal epilepsy for over a century now. Success rates remain steady around 40-60\% seizure freedom, even though candidates present more challenges nowadays than in the past [68], when most surgeries were in the mesiotemporal lobe. Many guidelines now stipulate that anyone with focal epilepsy who is resistant to two or more adequately dosed anti-epileptic drugs, should be considered for epilepsy surgery. The ideas why epilepsy surgery might work once seemed straightforward, but have come under scrutiny by new research findings, more sophisticated views of brain function, and the mystery mechanism in view of some unsuccessful cases.

Underlying surgery is the idea that the 'epileptic focus' should be removed. This was elaborated in the classical papers by Hans Lüders and co-workers [20, 74], who coined the term 'epileptogenic zone' (EZ), defined as the smallest area of cortex the removal of which will lead to seizure freedom. Being an abstraction, it is in practice approximated by the socalled 'seizure onset zone', usually situated within the 'irritative zone' of interictally abnormal cortex, showing spikes in the electroencephalography (EEG). These concepts emerged from experience with intracranial EEG recordings.

This way of thought has recently been expanded with the evolution of Magnetic Resonance Imaging (MRI), histological techniques and classification systems, leading to the belief that surgery is about removing pathological cortex in the anatomic sense, be it an evident lesion, or a microscopical deviation from the normal cortical layering [75]. Even in normal neuroimaging, in this view there should be a cortical substrate. Thus, the abstract 'epileptogenic zone' is replaced with the idea of a 'histologically pathogenic zone'. Surgical failure is then explained as incomplete removal of microscopical abnormalities.

In concurrence with these views, signal analysis led to the idea of epilepsy not as a localized, but as a network disease [69], with a collection of 'hyperexcitable nodes' in physiological networks. Concepts have been developed, partly from network theory, to describe and quantify these notions. We now speak of an 'epileptogenic network', network 'recruitment' and the development of 'dual pathology' or 'making the network epileptogenic', to explain disease progression or describe epileptogenesis. Networks are now also fashionable to explain surgical failures, seizure aggravation, deep brain stimulation and cognitive dysfunction that cannot be understood with only static, focal abnormalities in mind. Clinicians involved in epilepsy surgery will have to cope with these different views that challenge the framework that Lúders coined long ago. Still, surgeons operate on epilepsy patients when they see an anatomical abnormality and can pinpoint seizures to a nearby area if this is outside eloquent cortex. Thinking of networks then seems impractical because resection is ultimately focal.

We are now entering an age of advanced computer models that simulate the known physiological and pathological electrochemical properties of neuronal populations. Such neocortical focal epilepsy models mimic an EEG and show interictal spikes and focal seizures in the same unpredictable way as human epilepsy does. These computer models can account for local tissue properties as well as network influences. They can be personalized [6567] and therefore hold promise in counseling the neurosurgeon. At the conceptual level, we think that brain network analyses using such models may offer new ideas and strategies for epilepsy surgery. To illustrate this, we will use a relatively simple model of coupled neocortical 'nodes', showing both normal and seizure-like behavior, that may represent an underlying network model of the conceptual EZ and its surrounding tissue. By systematically 
studying arrangements of four nodes, we will show how network connections influence the seizure rate and how this might change our traditional views of epilepsy surgery, and raise new opportunities in surgical strategy.

\subsection{Methods}

\subsubsection{Model description}

Following others $[48,49,76]$, we consider a phenomenological computational network model consisting of nodes connected via directed edges. Each node models a population of neurons that produces an EEG-like signal. We consider each node in our network to represent a couple of squared centimeters of cortex, so part of a lobe. The dynamics of a node are described by a set of differential equations (see 2.S1). A node can produce two different types of activity, representing interictal and ictal activity. Interictal activity represents 'normal' brain activity and is characterized by noisy low-amplitude fluctuations. Ictal activity is modeled as pronounced $3 \mathrm{~Hz}$ oscillations, mimicking spike-wave discharges.

The simulated activity of nodes 1 and 2 in Figure 2.1A show the intrinsic dynamics of a node. Both nodes show alternating periods of interictal and ictal activity that arise without changing model parameters. The transition from interictal to ictal activity is due to stochastic perturbations in the model. The transition probability is regulated by a parameter representing the excitability of a node. Termination of ictal activity is nearly deterministic and regulated by a slow process (see 2.S1). In Figure 2.1A, node 2 is hyperexcitable, which results in more frequent transitions to ictal activity. In the same figure, the effect of coupling is demonstrated: node 4 receives input from node 3 . Consequently, node 4 shows ictal activity only if node 3 produces ictal activity.

\subsubsection{Study Design}

We investigate the role of hyperexcitable nodes in small networks and study the effect of surgery in those networks. We study all 218 topologically different network structures on four nodes. For each of these network structures we consider five networks: in one network all nodes have normal excitability, in the other four, one node is hyperexcitable. This yields 1090 different networks. To determine the networks that exhibit seizures we simulate 5 hours of activity for all networks. All well-connected networks with on average more than one seizure per hour are selected, where we define a seizure as three or four nodes producing ictal activity simultaneously.

We then try to decrease the seizure rate in the seizure networks by removing one of the nodes in these networks. The removal of a node thus mimics the effect of tissue removal in epilepsy surgery, and, if successful, would define the EZ. We evaluate the effect of removing a node by simulating the remaining network for another 5 hours and count the number of seizures in this new simulation. As the remaining network has three instead of four nodes we define a seizure in this reduced network as having two or three nodes in ictal state at the same time. Removing a node is only considered if the remaining network has some connections left. Using this procedure, we determine the optimal node to remove to reduce the seizure frequency. We compare this with the effect of removing the hyperexcitable node. 

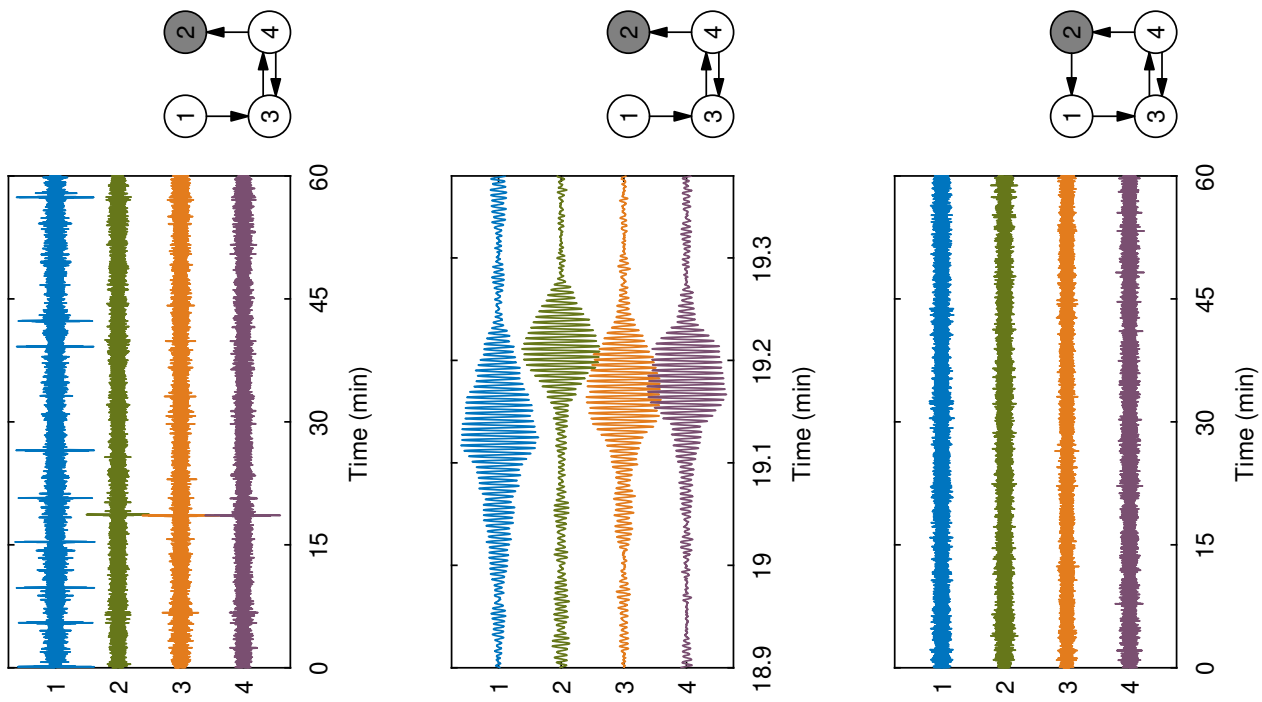

$\boldsymbol{m} \quad$ әроN

อ 2 อN

ᄂ
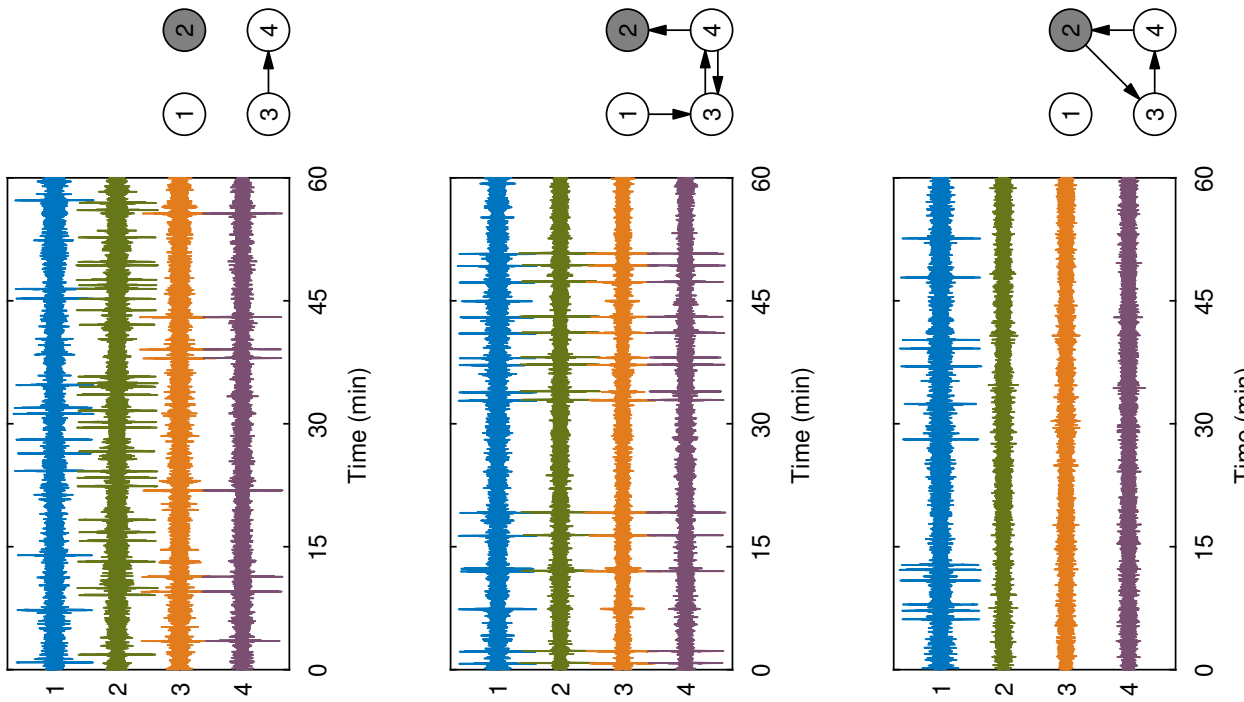

$\varangle$ әpoN

$\mathcal{3}$ әроN

Ш

Figure 2.1: (A-C, E, F) Simulations of five characteristic networks. The high amplitudes in the signals are periods of ictal activity. In these networks, the gray node (node 2) is hyperexcitable. (D) Close-up of a seizure in $\mathbf{C}$. The seizure starts at the driver (node 1 ) and spreads via nodes 3 and 4 to node 2 . 


\subsection{Results}

Figure 2.1 shows simulations for some typical networks. The network in Figure 2.1B has a reciprocal connection between nodes 3 and 4 . We call such a loop a 'cycle'. This cycle stabilizes the network. The network in Figure 2.1C is similar except for an additional connection $1 \rightarrow 3$. This connection causes a large increase in seizure rate. In this network all seizures start at node 1 (see Figure 2.1D), which is a node that does not receive input itself. We will call such a node a 'driver'. The networks in Figures 2.1E, F both contain cycles. Despite the presence of a hyperexcitable node in these cycles, they do not show seizures. This shows the importance of network structure: a hyperexcitable node is not necessarily bad, depending on the location in a network.

We found 387 networks with sufficient seizures of which 72 do not contain a hyperexcitable node. Histograms of the distribution of seizures are shown in Figure 2.2. Results suggest a categorization of networks in three classes: networks without a cycle, networks with cycle but without driver and networks with a cycle and a driver. The networks with cycle but without a driver exhibit only a few seizures. Networks in the other classes show many seizures.

Also the presence of a hyperexcitable node plays a role. The seizure distribution of networks with a hyperexcitable node shows an additional peak (Figure 2.2C) of high seizure rate as compared to the networks without such a node. Networks with a cycle only show relevant seizure activity if they contain a hyperexcitable node.

The effect of node removal is shown in Figures 2.2B, D, E and is usually large in networks containing a cycle and a driver. Most of them become (almost) seizure free under optimal resection strategy, but not necessarily due to the removal of the hyperexcitable node. The best intervention is to reduce a network to one without a driver (Figures 2.2F, G). Intervention may incidentally lead to seizure increase if removal of the hyperexcitable node in a cycle creates a driver, as in Figure 2.2H.

Node removal in networks with a cycle but without a driver yields only a small decrease in seizure rate, from their originally low seizure rate. Networks without a cycle cannot become seizure free and show in general little improvement. Only when they have a hyperexcitable node as a driver and show an extremely high seizure rate, removal or isolation of the hyperexcitable node will decrease the seizure rate drastically (Figure 2.2I).

\subsection{Discussion}

Our work suggests that the notion of network structure and connections may be more important than localizing the pathological node. Our model shows that removal of normal, 'driving' nodes, located at a crucial site within the network, is effective in preventing seizures, and would constitute the EZ in such a case. At the same time, removal of the abnormal, hyperexcitable node in the same network does not always help. Local hyperexcitability thus does not seem to be an obligatory feature of the EZ in network dynamics. This may help to understand the success of anteromesial temporal lobe surgery, in which the hippocampus may be such a driver. Removal of the hippocampus has become key to the effect of temporal lobe surgery, even in neocortical cases. It may also explain why lesionectomy in itself may not be sufficient, as a non-pathological driver in a network cycle may still remain.

Of course, computational models are a simplification. In our model the dynamical 

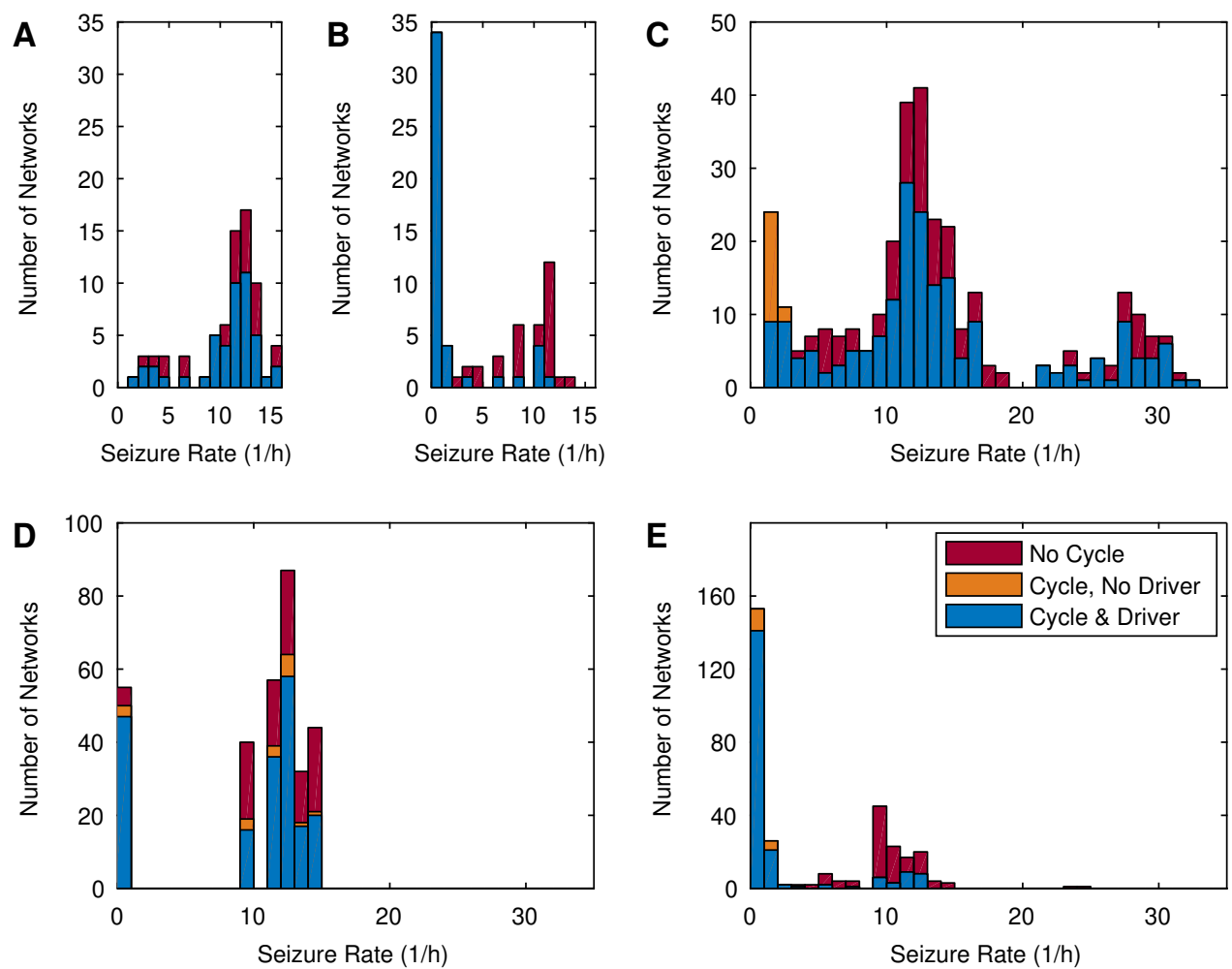

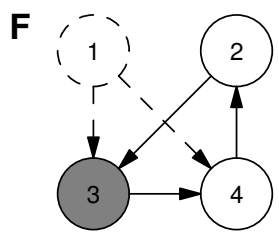

$12.6 \rightarrow 0(12.0)$
G

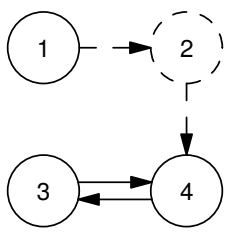

$12.8 \rightarrow 0.4(-)$
H

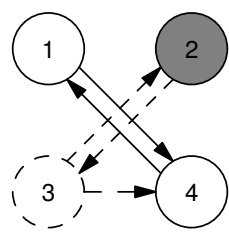

$1.6 \rightarrow 0.2(12.2)$

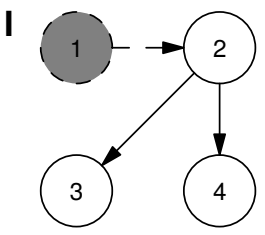

$28.4 \rightarrow 11.8(11.8)$

Figure 2.2: (A, C) Seizure rate distribution of the original networks for networks without and with a hyperexcitable node, respectively. (B, E) Seizure rate distribution after removal of the optimal node for normal networks with a hyperexcitable node, respectively. (D) Seizure rate distribution after removing the hyperexcitable node. (F-I) Optimal improvements in selected networks (indicated by dashed lines). The numbers below indicate initial seizure rate $\rightarrow$ seizure rate after optimal improvement (seizure rate after removal of hyperexcitable node). Optimal improvements in all other networks can be found in 2.S2.

repertoire of a node comprises an interictal and an ictal state. We assume that each single node may produce ictal activity. The intrinsic dynamics of a single node cannot be measured in vivo, as it is influenced by other nodes. Only in case of in vitro brain slices the dynamics of a single node can be observed. It has been reported that such slices will produce spontaneous epileptiform activity [77, 78]. The node dynamics can be made more sophisticated and 
realistic by using physiologically detailed neural mass models, that have been designed to describe a variety of activities as observed in EEG-signals [42].

A promising development is to taylor this approach to individual patients, e.g. those undergoing chronic invasive EEG monitoring, by using a patient-specific network model. Such networks can be derived from EEG data using functional connectivity measures, e.g. correlation or Granger causality. Information about local cortical excitability can be incorporated in the node parameters. The effect of removal of certain cortical areas could then be predicted using the computer [65-67], and this could be compared to clinical effect in a prospective study.

\section{S1 Detailed Model Description}

Following [48, 49, 76], we consider a phenomenological model of interictal and ictal dynamics. This model consists of nodes coupled through a directed graph. The dynamics of node $k$ is described by two variables: a complex activity variable $z_{k}$ and a real variable $\lambda_{k}$ that represents the excitability of the node. The observable quantity for each node is the real part of $z_{k}$, representing an EEG signal. Each node can produce two types of activity: noisy low-amplitude interictal activity and pronounced oscillations that represent ictal activity.
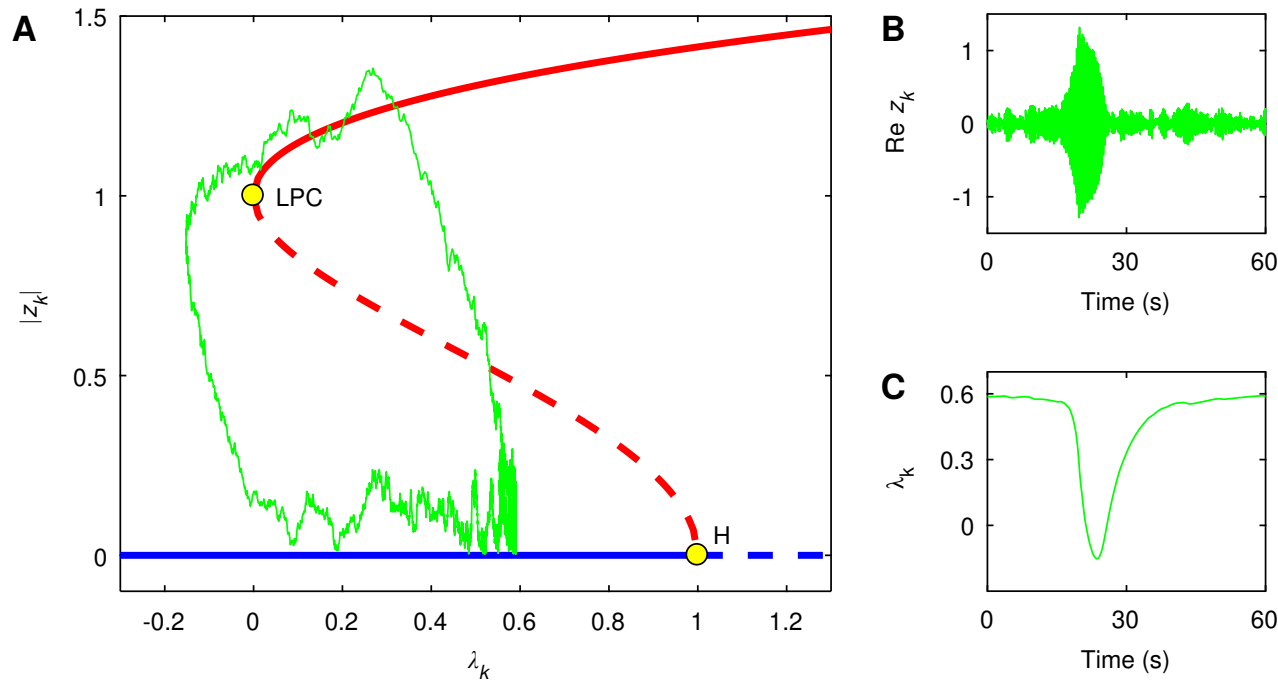

Figure 2.3: (A) Bifurcation diagram for $\left|z_{k}\right|$ as function of $\lambda_{k}$. The blue line indicates the equilibrium $z=0$. The red line indicate the limit cycle. Solid lines are stable solutions, dashed lines are unstable solutions. The green line shows variation of $\left|z_{k}\right|$ and $\lambda_{k}$ during a transition from interictal to ictal activity and back to interictal activity. (B, C) Time profiles of $\operatorname{Re}\left(z_{k}\right)$ and $\lambda_{k}$ during the same transition as in $\mathbf{A}$.

We will interpret these two activity types in terms of dynamical system properties by considering the dynamics of a single node as a slow-fast system. Studying the node dynamics as a slow-fast system is allowed since changes in the excitability $\lambda_{k}$ are slow compared to $z_{k}$. First we look at the dynamics of the fast system, i.e. the dynamics of $z_{k}$ for fixed $\lambda_{k}$. In this 
system, interictal activity arises from noisy perturbations around a stable equilibrium, while ictal activity is generated by a stable limit cycle. Figure $2.3 \mathrm{~A}$ shows a bifurcation diagram of the fast system. Here one can see that for $\lambda_{k}<1$ the fast system has a stable equilibrium at $z=0$, which is indicated by the solid blue line. At $\lambda_{k}=1$ a Hopf bifurcation occurs and the equilibrium becomes unstable for larger values of $\lambda_{k}$ (dashed blue line). From the Hopf bifurcation an unstable limit cycle emerges. This unstable limit cycle exists for $0<\lambda<1$. At $\lambda=0$, this unstable limit cycle changes via a limit point of cycles (LPC) bifurcation to a stable limit cycle. This stable limit cycle exists for $\lambda_{k}>0$. Both the unstable and stable limit cycle are a circle centered around $z=0$ in the complex plane. Their radii are indicated by the red line in the bifurcation diagram in Figure 2.3A. The frequency of the limit cycles is determined by a parameter $\omega$. In this study we set $\omega=20$. This yields a frequency of around $3 \mathrm{~Hz}$, comparable to the frequency of spike-wave discharges.

The speed of the slow dynamics is regulated by a time constant $\tau$. Motivated by the experimental work of [79], we choose $\tau=5 \mathrm{~s}$. If the fast system is in interictal state, the slow dynamics will try to move $\lambda_{k}$ towards $\lambda_{0, k}$. Therefore $\lambda_{0, k}$ can be seen as the base level of excitability. In this study we take $\lambda_{0, k}=0.6$ for a normal node and $\lambda_{0, k}=0.65$ for a more excitable node. For these values of $\lambda_{0, k}$, the system has a stable equilibrium at $z=0$ and $\lambda_{k}=\lambda_{0, k}$.

An important aspect in this model is that each node receives independent white noise input with strength $\alpha$ representing background input from unmodeled brain regions. In this study we take $\alpha=0.1$. The noise perturbs the system around the stable equilibrium state. This is visible as the noisy perturbations seen in the interictal activity. However, if $0<\lambda_{0, k}<1$, the noisy input may force $z$ to jump to the stable limit cycle, representing the ictal activity. As a reaction $\lambda_{k}$ will slowly decrease. During this process the fast $z_{k}$ activity will adapt immediately. When $\lambda_{k}$ gets smaller than zero, the stable limit cycle of the fast dynamics disappears and the fast system jumps back to the interictal state. At this moment the slow $\lambda_{k}$ will increase again to the base level $\lambda_{0, k}$. In Figure $2.3 \mathrm{~A}$ an example of a transition from interictal to ictal activity and the subsequent transition to interictal activity is indicated in the $\lambda_{k}-\left|z_{k}\right|$-plane by the green line. The corresponding time series for the output variable $\operatorname{Re}\left(z_{k}\right)$ and the excitability $\lambda_{k}$ are shown in Figures $2.3 \mathrm{~B}, \mathrm{C}$, respectively.

We note that when the system is in interictal state and $\lambda_{k}$ is at its resting value, then the escape time from interictal activity to ictal activity is stochastic and approximately exponentially distributed [49]. This escape time decreases if the base level of excitation $\lambda_{0, k}$ or the noise strength $\alpha$ is increased [49]. The duration of an episode of ictal activity is approximately deterministic of length, since this depends mainly on the slow process and its time constant $\tau$. The same holds for the time needed to get back from low excitation level to the base level excitation after an episode of ictal activity.

Besides background input, each node also receives input from other nodes. This input is modeled as diffusive coupling on a simple directed graph, which means that it influences the activity of the receiving node to move towards the activity of the projecting node. The directed graph can be described by an adjacency matrix $M$. In this matrix $\mathbf{M}_{k l}=1$ if there is a connection from node $l$ to node $k$ (and thus node $k$ receives input from node $l$ ) and $\mathrm{M}_{k l}=0$ if there is no connection. We exclude self-connections, so $\mathrm{M}_{k k}=0$ for all $k$, since we assume that the influence of self-connections can be represented by the intrinsic dynamics of a node. The strength of all connections is given by a global constant $\beta$. In this study we set $\beta=0.4$.

The description above leads to the following set of Itô-type stochastic differential equa- 
tions:

$$
\begin{aligned}
\mathrm{d} z_{k} & =\left(z_{k}\left(\lambda_{k}-1+i \omega+2\left|z_{k}\right|^{2}-\left|z_{k}\right|^{4}\right)+\beta \sum_{l=1}^{4} M_{k l}\left(z_{l}-z_{k}\right)\right) \mathrm{d} t+\alpha \mathrm{d} W_{k}, \\
\tau \mathrm{d} \lambda_{k} & =\left(\lambda_{0, k}-\lambda-\left|z_{k}\right|^{2}\right) \mathrm{d} t,
\end{aligned}
$$

where the nodes are numbered $k=1, \ldots, 4$. We compute solutions to this system of stochastic differential equations using an Euler-Maruyama scheme with time steps of 0.0001 seconds.

\section{S2 Selected Seizure Networks}

Figure 2.4 shows all the 387 selected four node networks. All these networks exhibited on average more than one seizure per hour during 5 hours of simulation. For each network a graycoloured node indicates a hyperexcitable node. A node with a dashed boundary indicates the optimal node to remove in order to decrease the seizure rate as much as possible. Connections from/to this node are dashed too, as these connections are automatically removed if the node is removed. In some networks there is no node whose removal leads to decrease in seizure rate and hence all nodes are drawn with solid lines. Below each network three numbers are displayed in the form $x \rightarrow y(z)$. Here $x$ denotes the seizure rate of the original network, $y$ of the optimal improved network and $z$ the seizure rate of the network that is obtained by removing the hyperexcitable node (if applicable). The number 'Sn. no.' above the network denotes the index of the selected seizure network and 'Graph no' denotes a number that is used to encode the network structure.

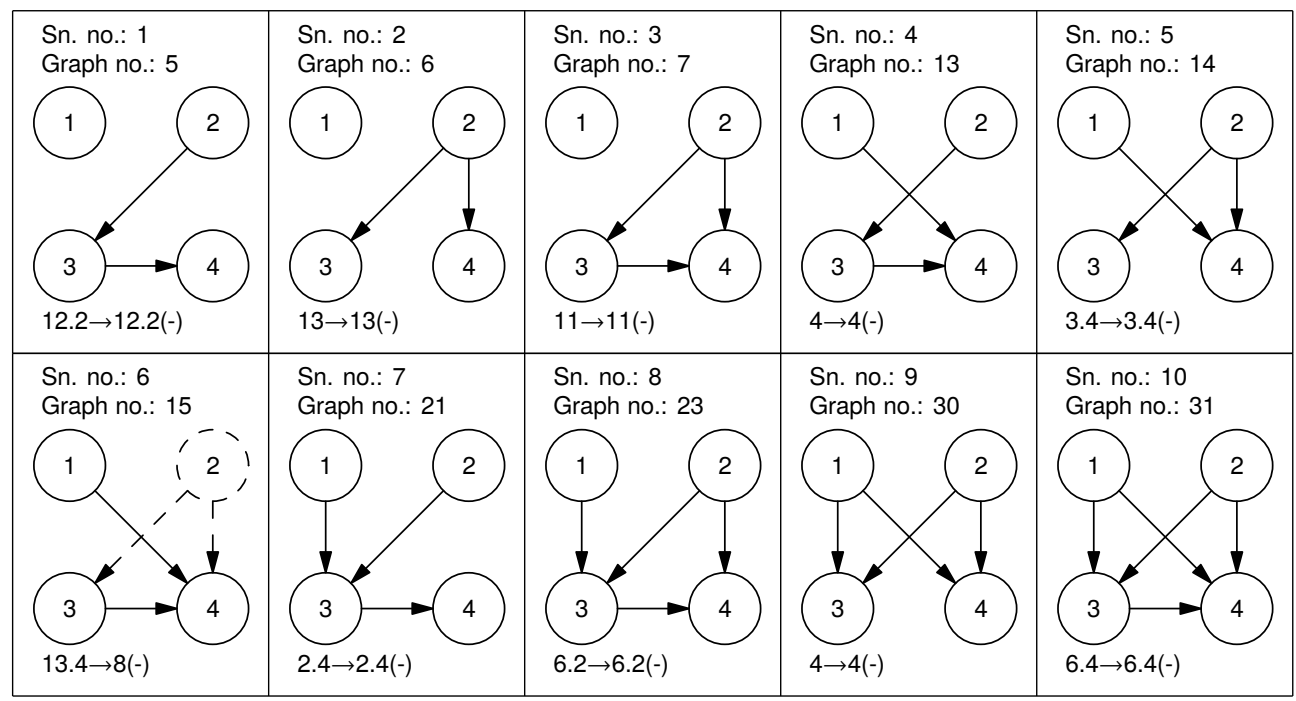

Figure 2.4: (continues on next page) 


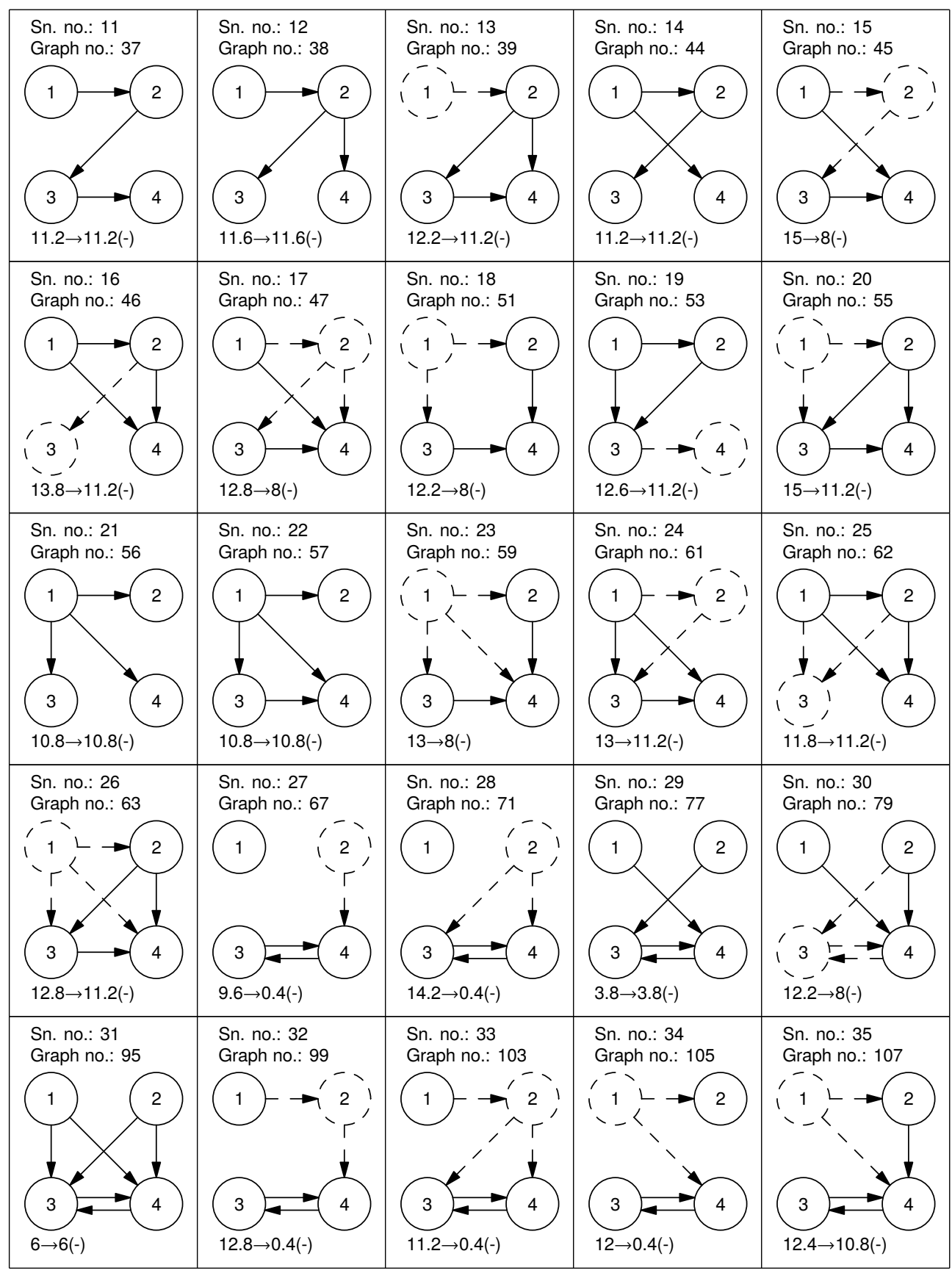

Figure 2.4: (continues on next page) 


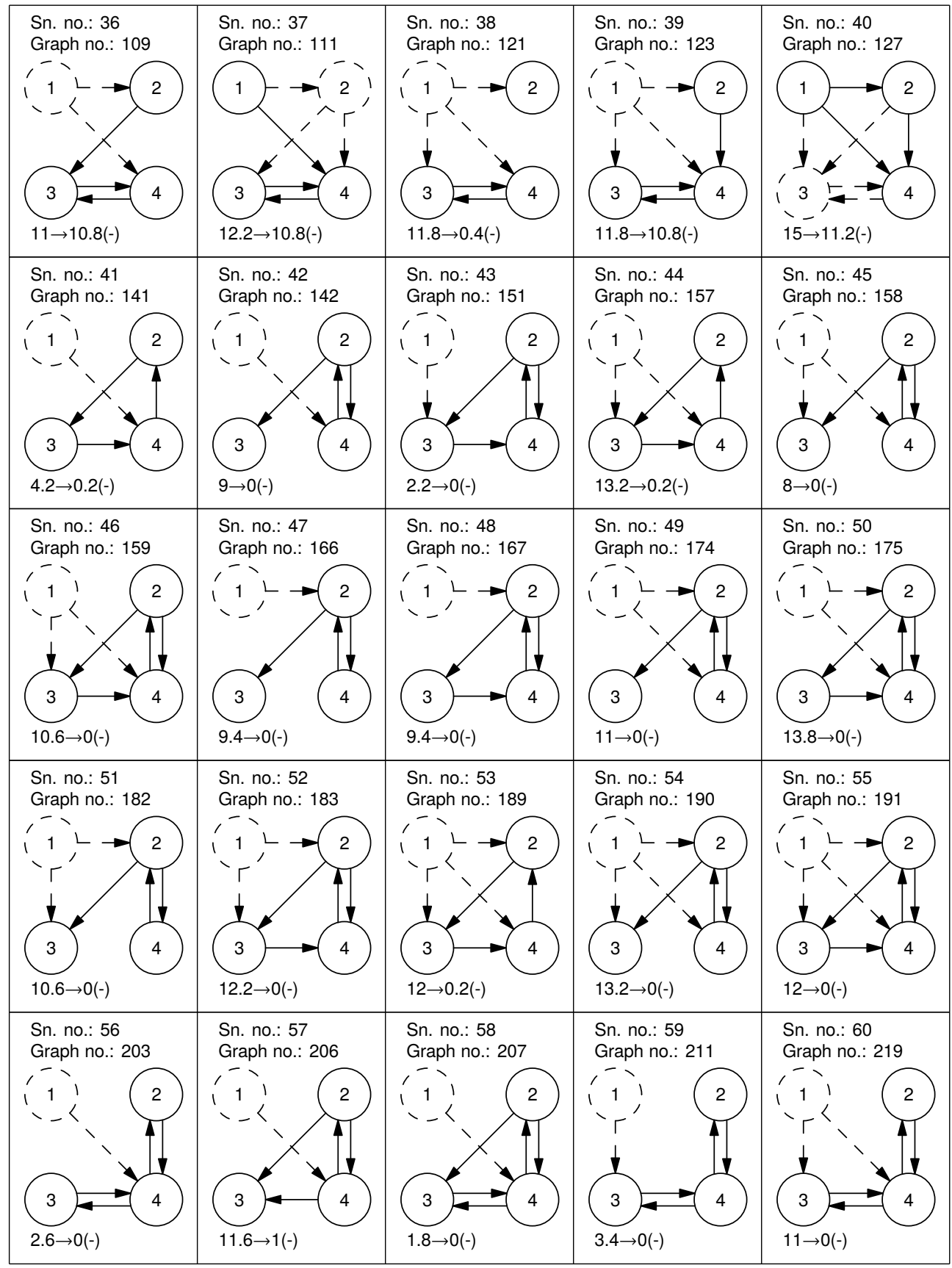

Figure 2.4: (continues on next page) 


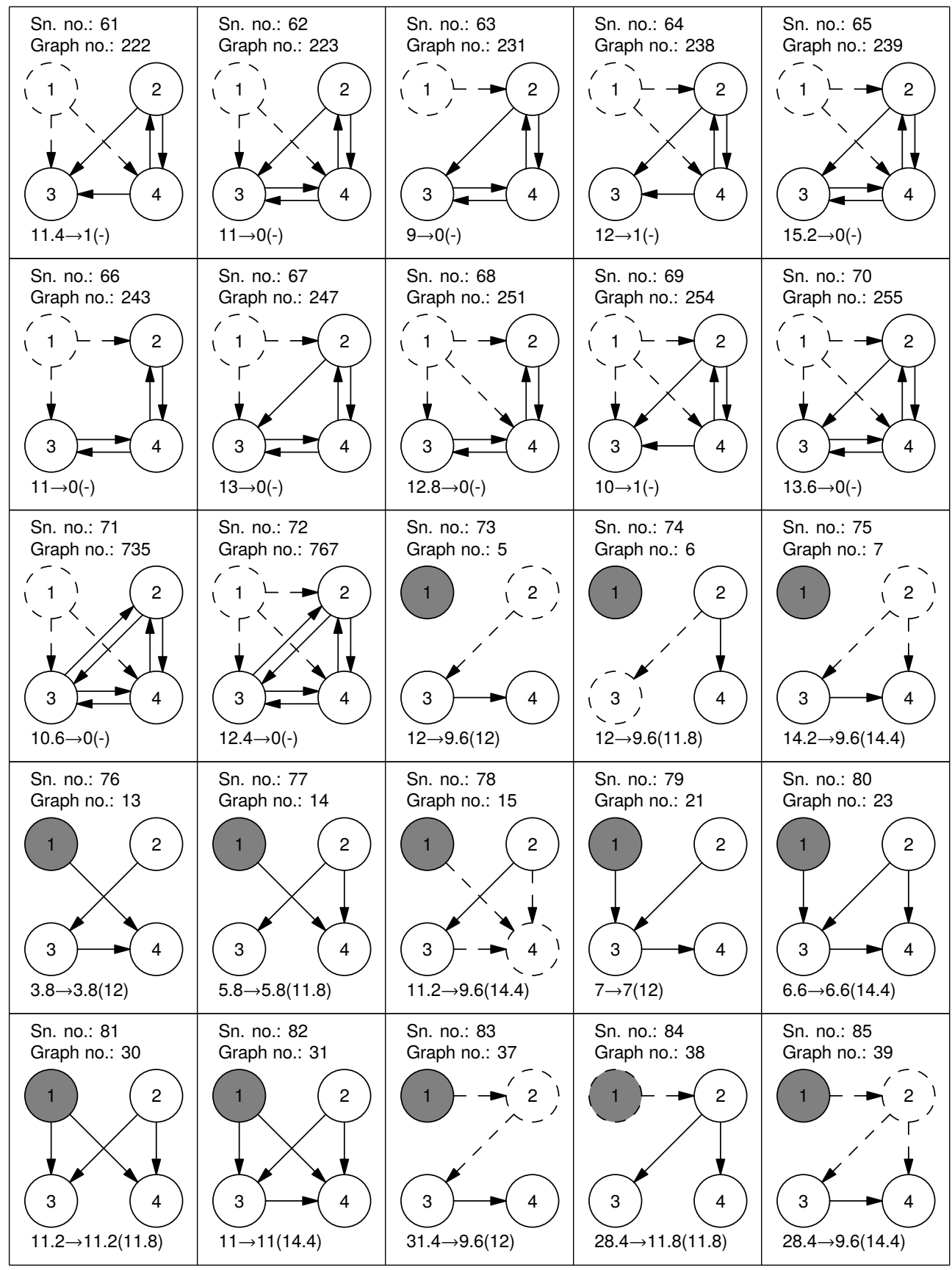

Figure 2.4: (continues on next page) 


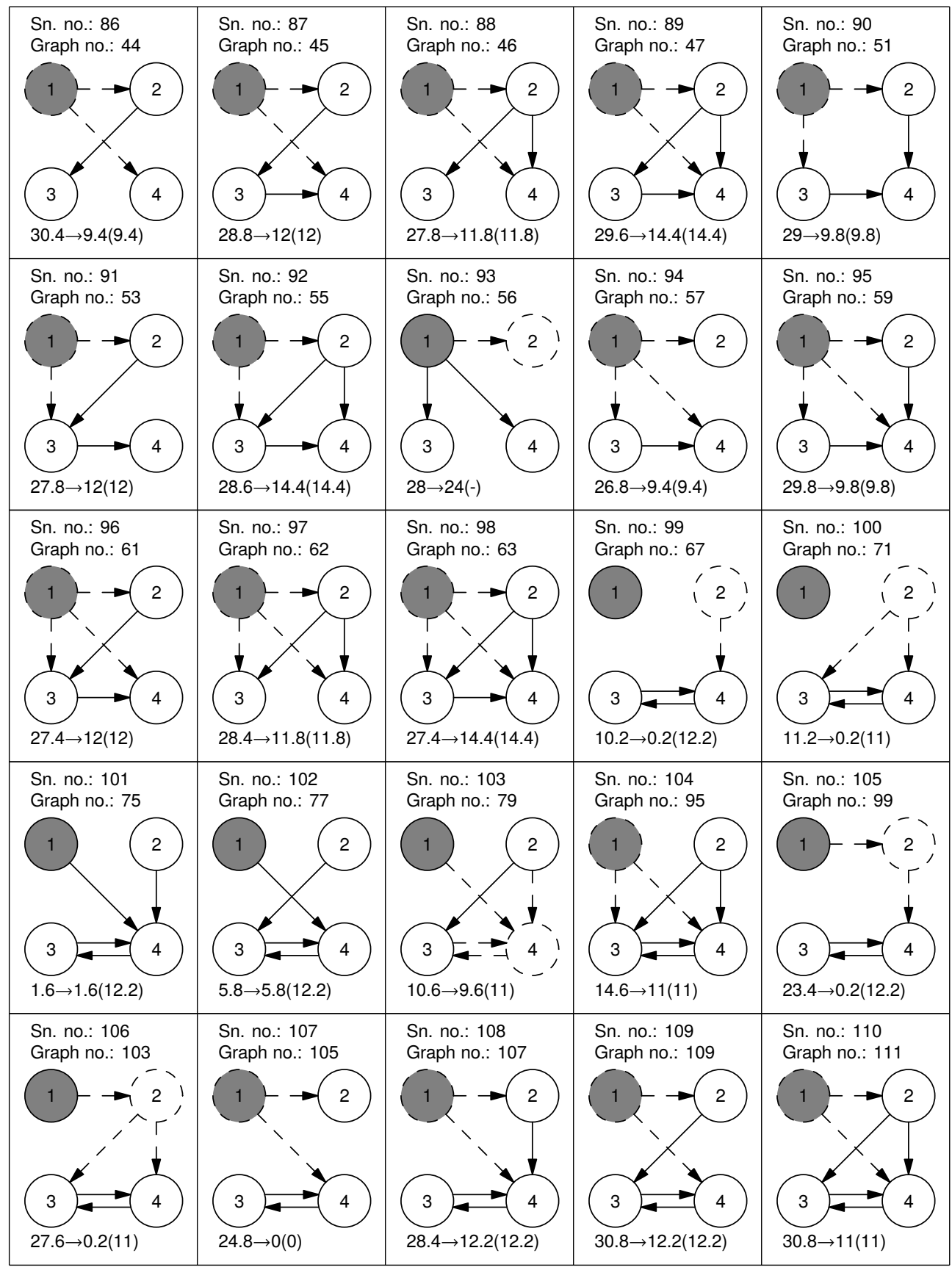

Figure 2.4: (continues on next page) 


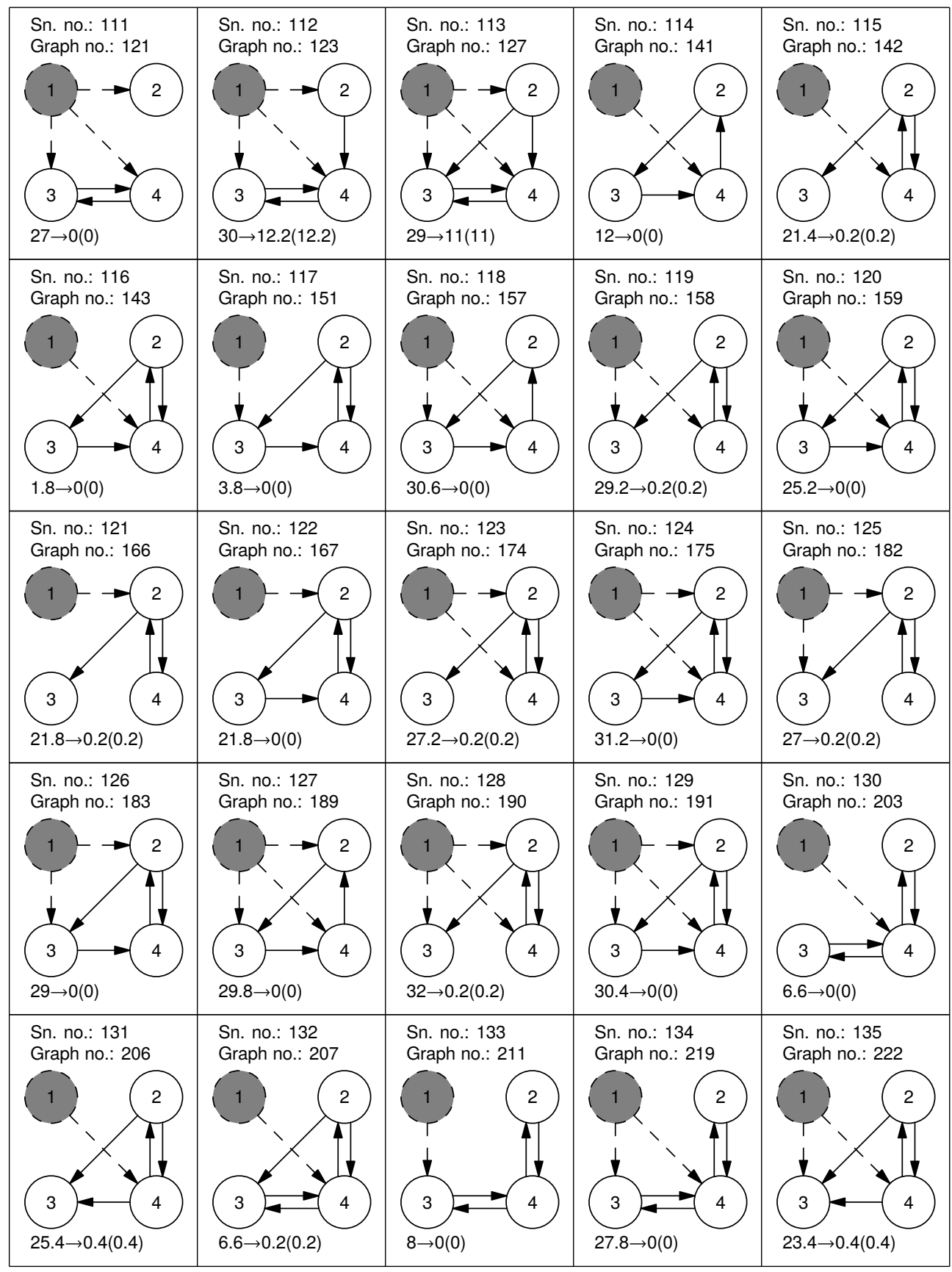

Figure 2.4: (continues on next page) 


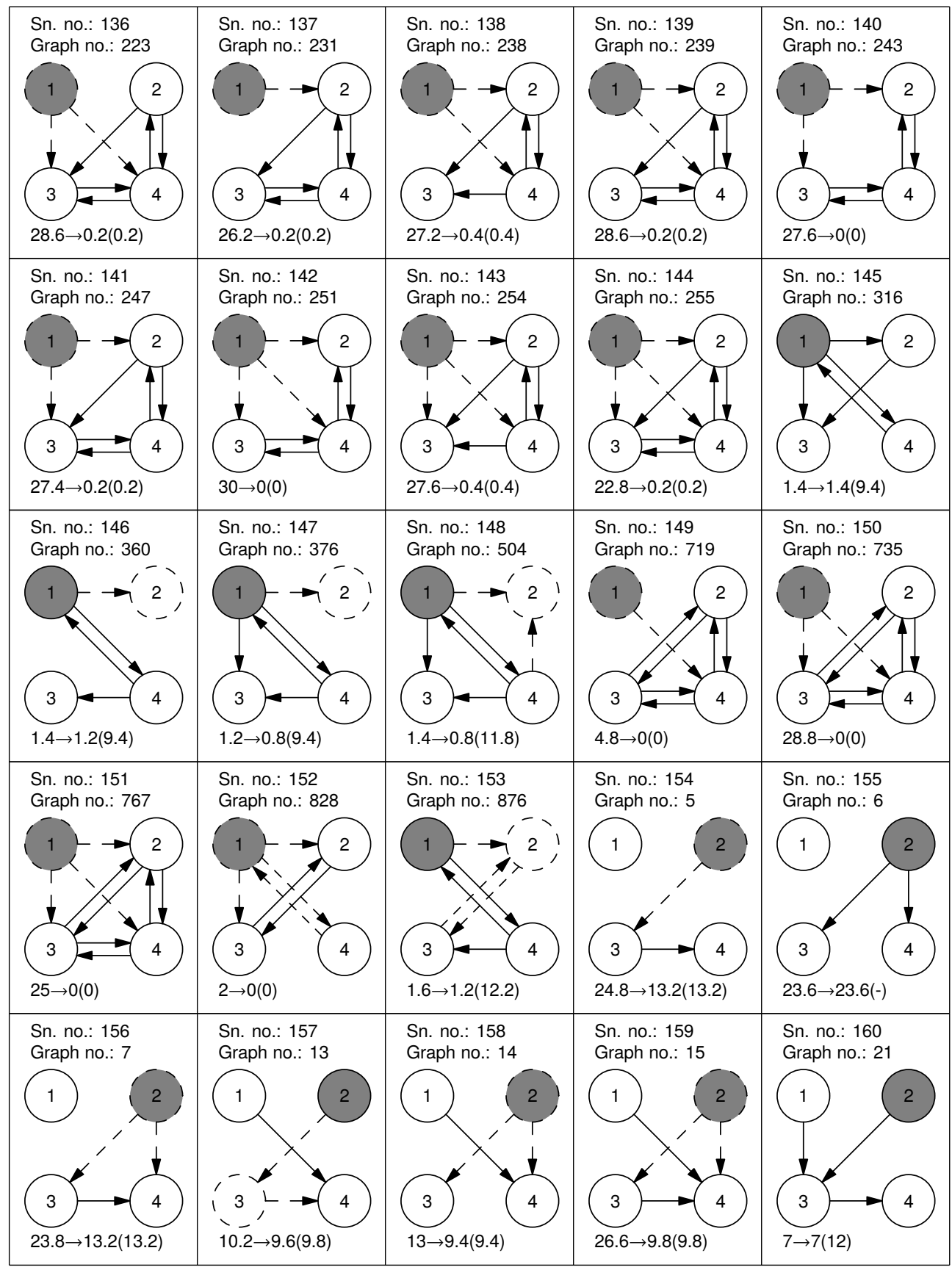

Figure 2.4: (continues on next page) 


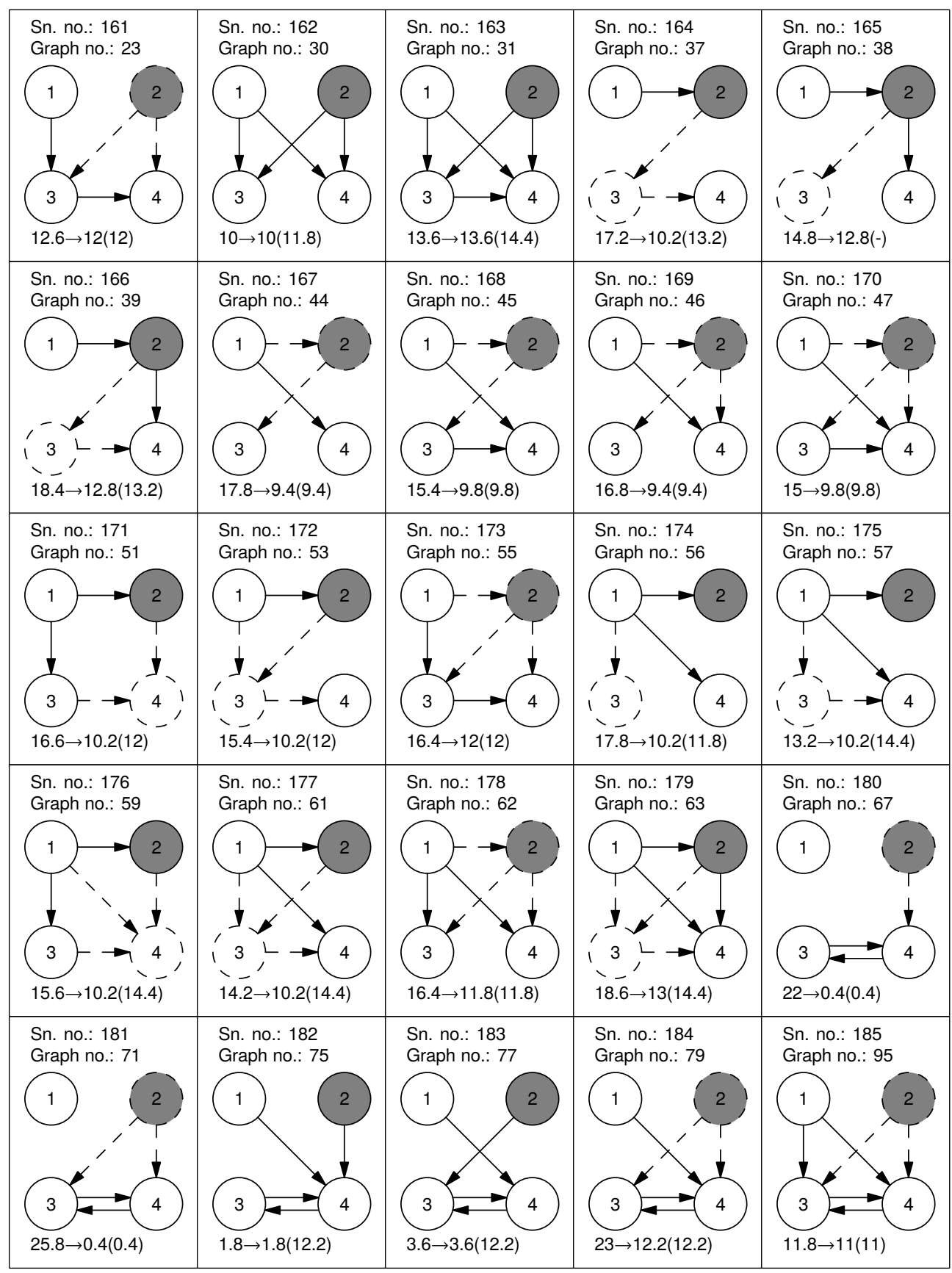

Figure 2.4: (continues on next page) 


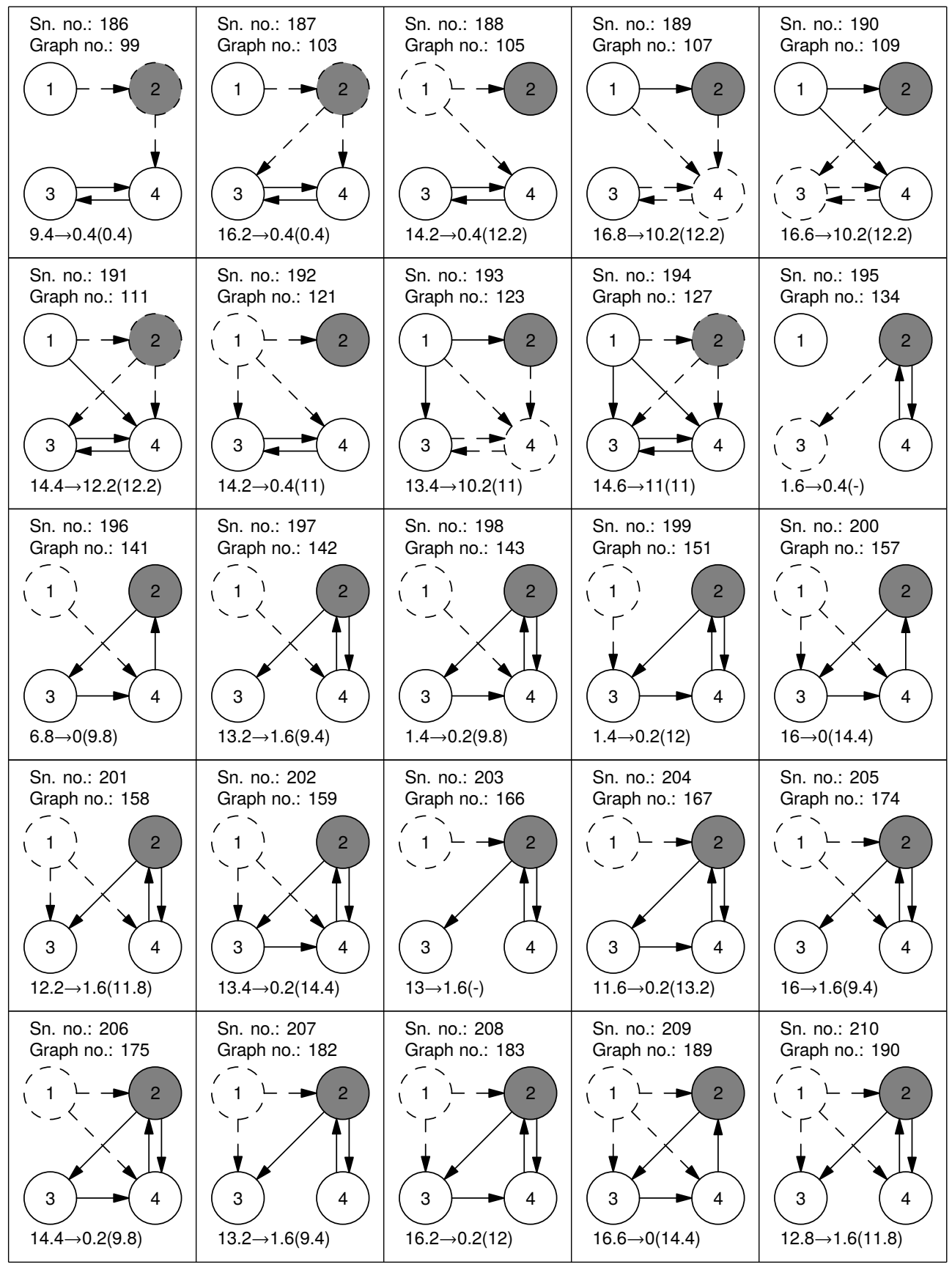

Figure 2.4: (continues on next page) 


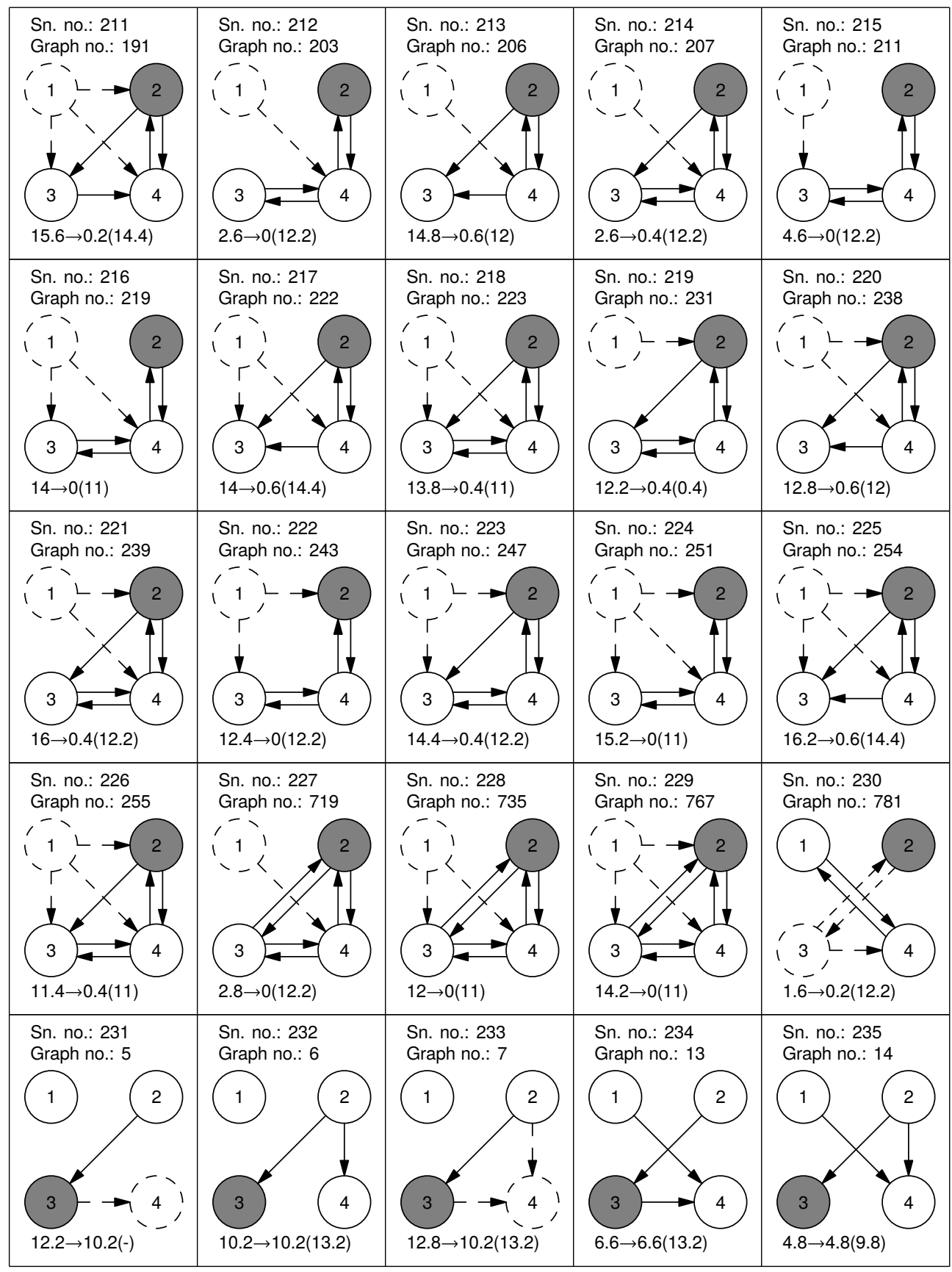

Figure 2.4: (continues on next page) 


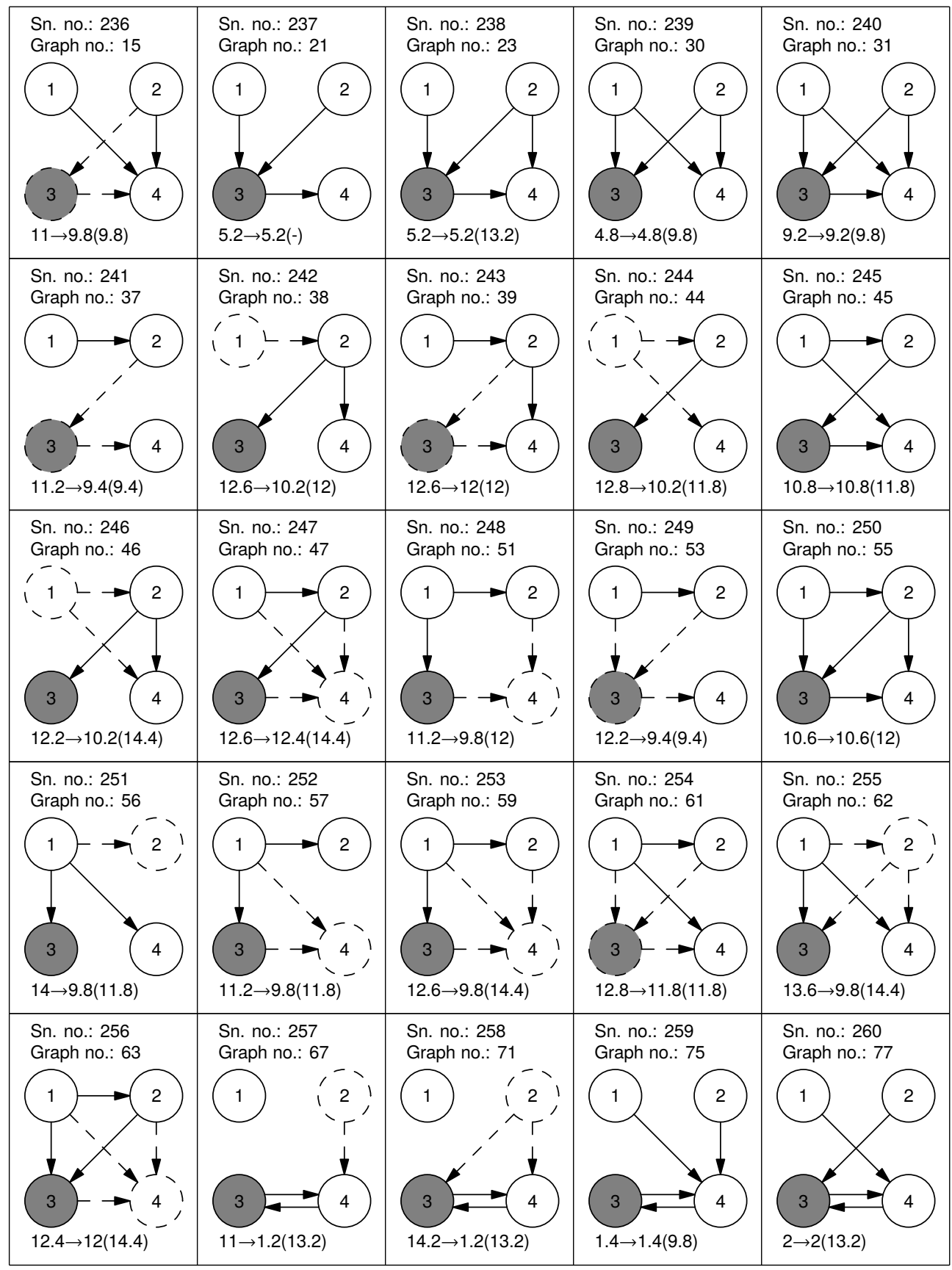

Figure 2.4: (continues on next page) 


\begin{tabular}{|c|c|c|c|c|}
\hline $\begin{array}{l}\text { Sn. no.: } 261 \\
\text { Graph no.: } 79\end{array}$ & Gn. no.: 262 & $\begin{array}{l}\text { Sn. no.: } 263 \\
\text { Graph no.: } 99\end{array}$ & Sn. no.: 264 & $\begin{array}{l}\text { Sn. no.: } 265 \\
\text { Graph no.: } 105\end{array}$ \\
\hline $\begin{array}{l}\text { Sn. no.: } 266 \\
\text { Graph no.: } 107\end{array}$ & $\begin{array}{l}\text { Sn. no.: } 267 \\
\text { Graph no.: } 109\end{array}$ & (3) & $\begin{array}{l}\text { Sn. no.: } 269 \\
\text { Graph no.: } 121\end{array}$ & $\begin{array}{lll}\text { Sn. no.: } 270 \\
\text { Graph no.: } 123\end{array}$ \\
\hline $\begin{array}{l}\text { Sn. no.: } 271 \\
\text { Graph no.: } 127\end{array}$ & $\begin{array}{l}\text { Sn. no.: } 272 \\
\text { Graph no.: } 141\end{array}$ & $\underbrace{}_{10 \rightarrow 0.6(12.2)}$ & $\underbrace{}_{2.2 \rightarrow 0(0.4)}$ & $\begin{array}{l}\text { Sn. no.: } 275 \\
\text { Graph no.: } 157\end{array}$ \\
\hline $\begin{array}{l}\text { Sn. no.: } 276 \\
\text { Graph no.: } 158\end{array}$ & $\begin{array}{l}\text { Sn. no.: } 277 \\
\text { Graph no.: } 159\end{array}$ & $\begin{array}{l}\text { Sn. no.: } 278 \\
\text { Graph no.: } 166\end{array}$ & $\begin{array}{l}\text { Sn. no.: } 279 \\
\text { Graph no.: } 167\end{array}$ & $\begin{array}{l}\text { Sn. no.: } 280 \\
\text { Graph no.: } 174\end{array}$ \\
\hline $\begin{array}{l}\text { Sn. no.: } 281 \\
\text { Graph no.: } 175 \\
1<\rightarrow\end{array}$ & $\begin{array}{l}\text { Sn. no.: } 282 \\
\text { Graph no.: } 182\end{array}$ & $\begin{array}{l}\text { Sn. no.: } 283 \\
\text { Graph no.: } 183\end{array}$ & $\begin{array}{l}\text { Sn. no.: } 284 \\
\text { Graph no.: } 189\end{array}$ & $\begin{array}{l}\text { Sn. no.: } 285 \\
\text { Graph no.: } 190\end{array}$ \\
\hline
\end{tabular}

Figure 2.4: (continues on next page) 


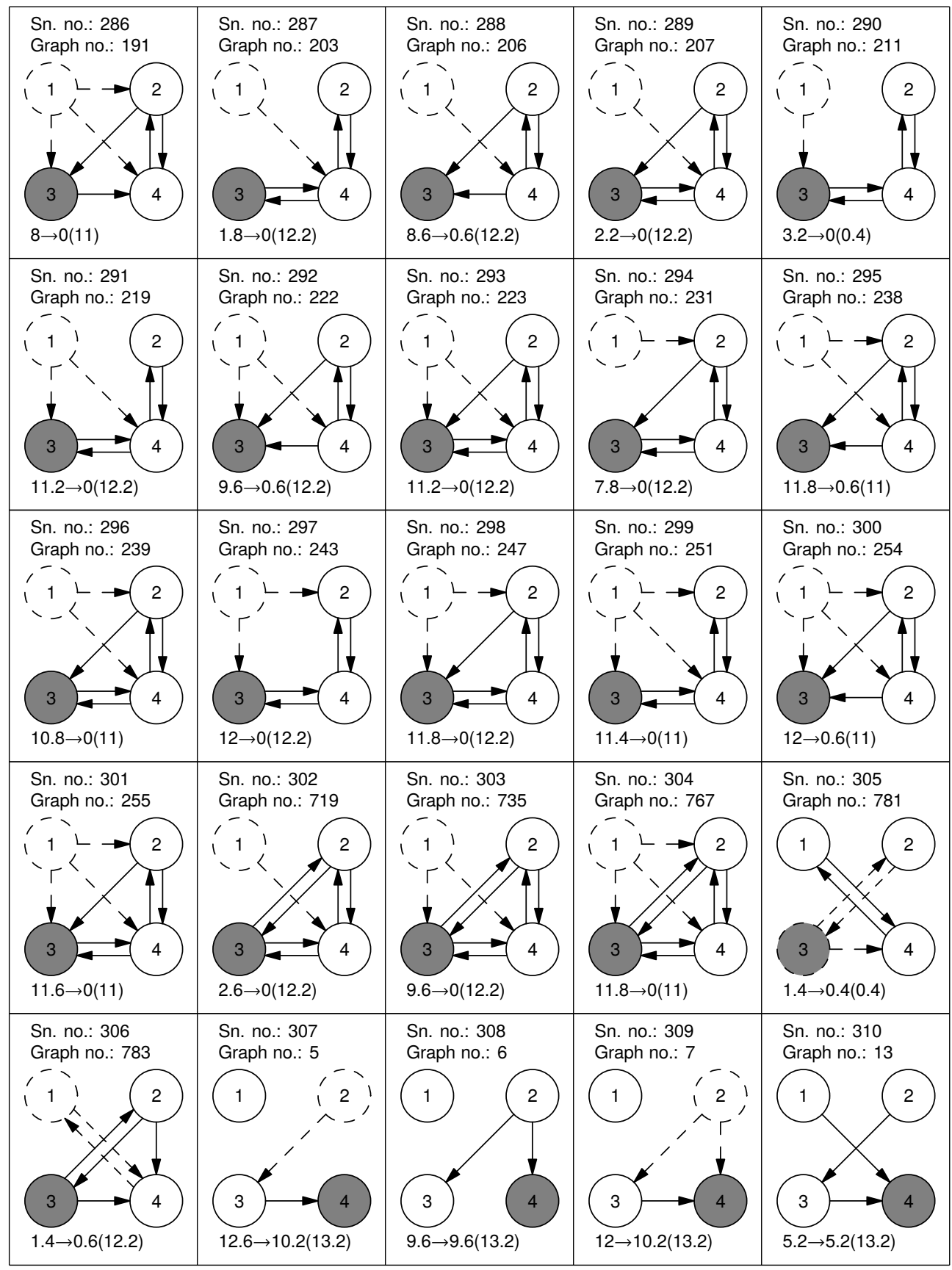

Figure 2.4: (continues on next page) 


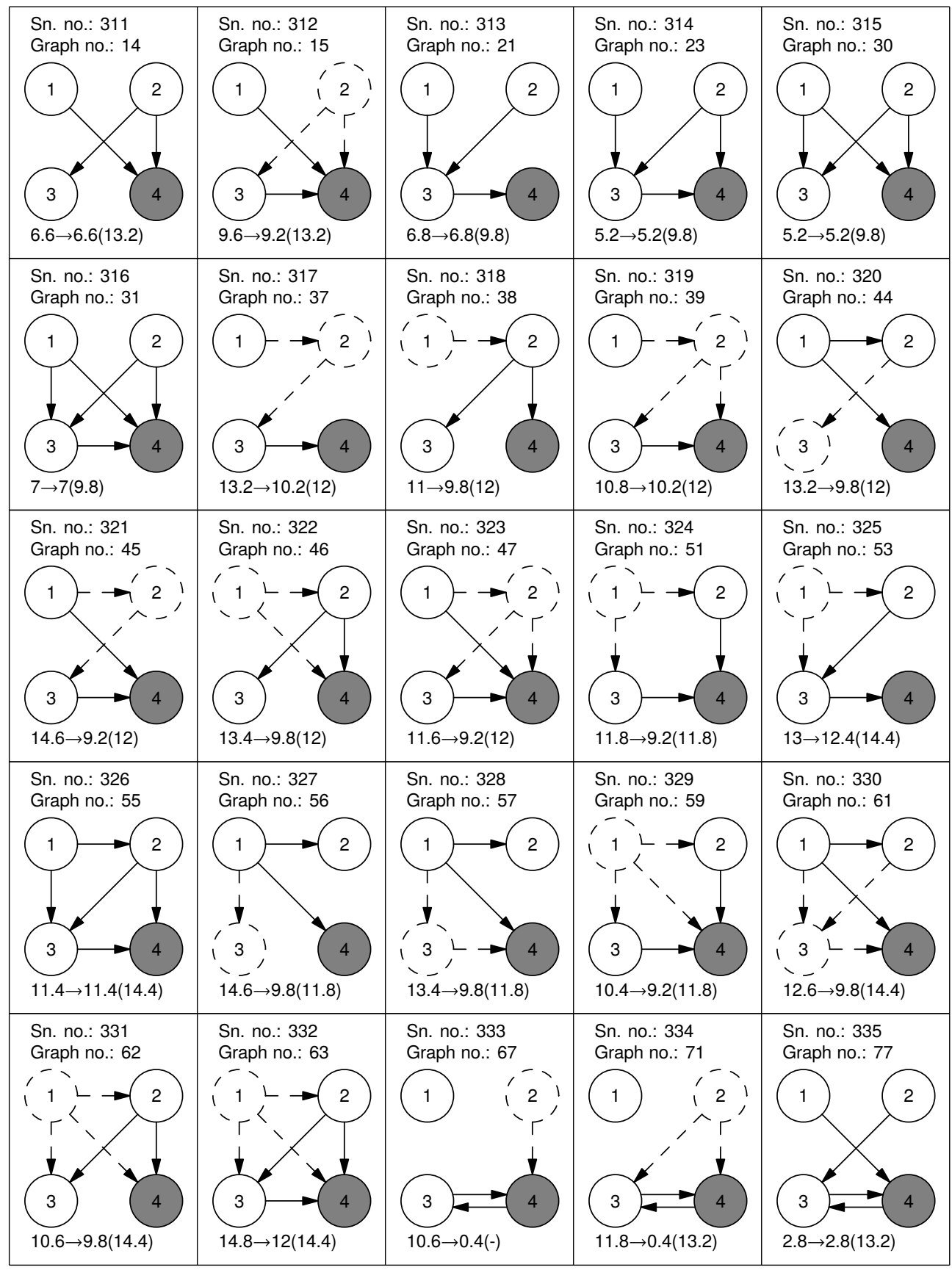

Figure 2.4: (continues on next page) 


\begin{tabular}{|c|c|c|c|c|}
\hline $\begin{array}{l}\text { Sn. no.: } 336 \\
\text { Graph no.: } 79\end{array}$ & $\begin{array}{l}\text { Sn. no.: } 337 \\
\text { Graph no.: } 95\end{array}$ & $\begin{array}{l}\text { Sn. no.: } 338 \\
\text { Graph no.: } 99\end{array}$ & $\begin{array}{l}\text { Sn. no.: } 339 \\
\text { Graph no.: } 103\end{array}$ & $\begin{array}{l}\text { Sn. no.: } 340 \\
\text { Graph no.: } 105\end{array}$ \\
\hline $\begin{array}{ll}\text { Sn. no.: } 341 \\
\text { Graph no.: } 107\end{array}$ & $\begin{array}{l}\text { Sn. no.: } 342 \\
\text { Graph no.: } 109\end{array}$ & $\begin{array}{l}\text { Sn. no.: } 343 \\
\text { Graph no.: } 111\end{array}$ & Sn. no.: 344 & $\begin{array}{l}\text { Sn. no.: } 345 \\
\text { Graph no.: } 123\end{array}$ \\
\hline 12.6 $\rightarrow 12(14.4)$ & $\begin{array}{l}\text { Sn. no.: } 347 \\
\text { Graph no.: } 134\end{array}$ & $\begin{array}{l}\text { Sn. no.: } 348 \\
\text { Graph no.: } 141 \\
1\end{array}$ & \begin{tabular}{l} 
Sn. no.: 349 \\
Graph no.: 142 \\
\hdashline 1
\end{tabular} & $\begin{array}{l}\text { Sn. no.: } 350 \\
\text { Graph no.: } 143\end{array}$ \\
\hline $\begin{array}{l}\text { Sn. no.: } 351 \\
\text { Graph no.: } 151\end{array}$ & $\begin{array}{l}\text { Sn. no.: } 352 \\
\text { Graph no.: } 157\end{array}$ & $\begin{array}{l}\text { Sn. no.: } 353 \\
\text { Graph no.: } 158 \\
1\end{array}$ & $\begin{array}{l}\text { Sn. no.: } 354 \\
\text { Graph no.: } 159\end{array}$ & $\begin{array}{l}\text { Sn. no.: } 355 \\
\text { Graph no.: } 166\end{array}$ \\
\hline $\begin{array}{l}\text { Sn. no.: } 356 \\
\text { Graph no.: } 167\end{array}$ & $\begin{array}{l}\text { Sn. no.: } 357 \\
\text { Graph no.: } 174\end{array}$ & $\begin{array}{l}\text { Sn. no.: } 358 \\
\text { Graph no.: } 175 \\
-1 L \rightarrow\end{array}$ & $\begin{array}{l}\text { Sn. no.: } 359 \\
\text { Graph no.: } 182\end{array}$ & $\begin{array}{l}\text { Sn. no.: } 360 \\
\text { Graph no.: } 183\end{array}$ \\
\hline
\end{tabular}

Figure 2.4: (continues on next page) 


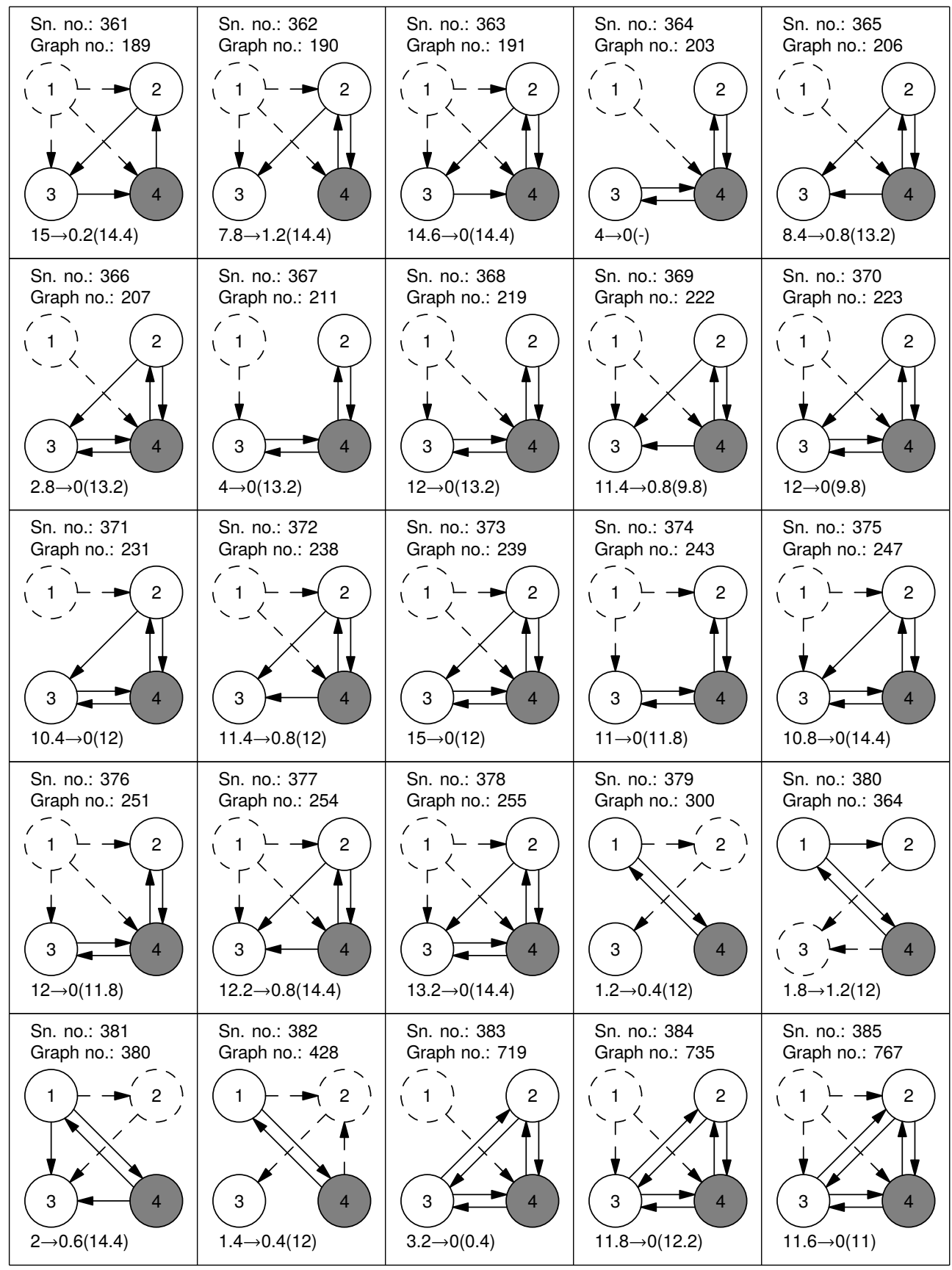

Figure 2.4: (continues on next page) 


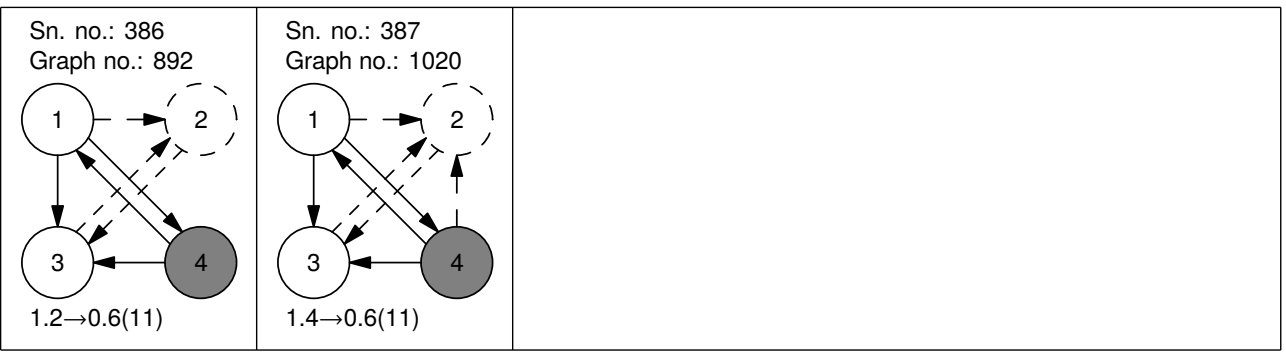

Figure 2.4: All selected networks on four nodes. Gray nodes indicate hyperexcitable node, while dashed lines indicate the optimal improvement. The numbers below indicate initial seizure rate $\rightarrow$ seizure rate after optimal improvement (seizure rate after removal of hyperexcitable node). 


\section{Chapter 3}

\section{A Comparison of Evoked and Non-Evoked Functional Networks}

Jurgen Hebbink, Dorien van Blooijs, Geertjan Huiskamp, Frans Leijten, Stephan van Gils, Hil Meijer 


\section{Abstract}

The growing interest in brain networks to study the brain's function in cognition and diseases has produced an increase in methods to extract these networks. Typically, each method yields a different network. Therefore, one may ask what the resulting networks represent.

To address this issue we consider electrocorticography (ECoG) data where we compare three methods. We derive networks from ongoing ECoG data using two traditional methods: Cross-correlation (CC) and Granger causality (GC). Next, connectivity is probed actively using Single pulse electrical stimulation (SPES). We compare the overlap in connectivity between these three methods as well as their ability to reveal well-known anatomical connections in the language circuit.

We find that strong connections in the CC network form more or less a subset of the SPES network. GC and SPES are related more weakly, although GC connections coincide more frequently with SPES connections compared to non-existing SPES connections. Connectivity between the two major hubs in the language circuit, Broca's and Wernicke's area, is only found in SPES networks.

Our results are of interest for the use of patient-specific networks obtained from ECoG. In epilepsy research, such networks form the basis for methods that predict the effect of epilepsy surgery. For this application SPES networks are interesting as they disclose more physiological connections compared to CC and GC networks. 


\subsection{Introduction}

Brain networks are increasingly being studied as they may aid in understanding the brain's function in cognition [80, 81] and diseases, such as Alzheimer's disease [82], epilepsy [83-85] and schizophrenia [86]. A recent development is to incorporate brain networks in computational models for epilepsy surgery $[65,66,87]$. Networks consist of nodes, representing neuronal populations, which are connected via edges. Based on the interpretation of the edges networks can be categorized as structural, functional or effective [88]. The concept of structural networks is the most intuitive; edges simply describe anatomical connections between neuronal populations. The presence of such an anatomical connection, however, does not indicate how intensively it is used in communication between the neuronal populations. Functional and effective connectivity methods try to assess this point. In functional connectivity edges describe statistical dependencies among time series of neuronal activity [89], while effective connectivity is defined as the influence one neuronal system exerts over another [90].

Methods for functional connectivity use simultaneously recorded time series which can be acquired via a large variety of imaging modalities, e.g. electroencephalography (EEG). Connectivity is then calculated from the band-filtered time series or their envelopes [91] using methods like cross-correlation (CC) [92], Granger causality (GC) [93] and mutual information [94]. Almost all these methods have a mathematical foundation that makes assumptions about the processes underlying the observations [89]. In practice, most of these assumptions only hold to some extent and one may wonder how this influences the obtained connectivity

Interventional approaches, in contrast, actively perturb activity at some location using electric or magnetic pulses in order to observe neural responses at other sites [33] and hence they infer connectivity in a more direct way than non-interventional approaches. Networks derived in this way are called evoked effective networks [33]. Pre-surgical evaluation of refractory focal epilepsy patients offers a unique setting to apply this approach in an invasive setting. In these patients electrocorticography (ECoG), i.e. an invasive form of EEG, may be recorded using an electrode grid placed directly on the cortex. Single Pulse Electrical Stimulation (SPES) [36] applies brief electric pulses to adjacent pairs of electrodes of this grid. These pulses have a typical duration of 0.1-3 ms and a strength of 2-12 $\mathrm{mA}$ [95] and evoke responses, called Cortico-Cortical Evoked Potentials (CCEP), at the non-stimulated electrodes. Commonly, two types of responses are distinguished in SPES literature, i.e. Early Responses (ERs) and Delayed Responses (DRs) [28]. ERs occur within $100 \mathrm{~ms}$. It is widely thought that they represent direct cortico-cortical propagation $[32,34,96]$. For completeness, we mention that DRs are typical for epileptogenic tissue [28, 40].

SPES offers a more direct approach to infer networks than functional connectivity. Functional connectivity, however, can be applied to recordings of ongoing ECoG activity as well as to non-invasive imaging methods like scalp EEG making it more accessible than SPES. While relations between structural and evoked effective networks have been studied [97-99], it is not known what functional networks constructed using ongoing ECoG have in common with SPES-evoked connectivity. Do they find the same connections? Do they reveal well-known anatomical connections?

To answer these questions we will construct networks for six patients using three different methods. One is the SPES network while the other two are CC and GC networks both derived from ongoing interictal ECoG. We will compare the connections between these networks and 
investigate to what extent those networks can unravel connectivity in an established functional network, i.e. the language circuit containing Broca's and Wernicke's area.

\subsection{Materials and Methods}

\subsubsection{Data selection \& pre-processing}

We use ECoG data, recorded with grid electrodes, of six patients with focal epilepsy who underwent long-term ECoG monitoring prior to surgery at the University Medical Centre Utrecht. Data are retrospectively studied and handled coded and anonymously according to the guidelines of the institutional ethical committee. Patient characteristics are provided in Table 3.1. For each patient, SPES has been performed as part of clinical routine. ECoG data has been recorded using a common reference montage with respect to an extracranial reference electrode. We consider two subsets of ECoG data for each patient: a segment of ongoing interictal data, to calculate functional connectivity, and the segment with SPES data.

The segments of ongoing ECoG data have been recorded just preceding SPES. In this way we are sure that effects of anti-epileptic drugs and situational confounders are similar for the ongoing ECoG and SPES recordings, while any influence of SPES on the connectivity for CC and GC is excluded. We note that by imposing this condition it was not possible to control the cognitive state of the patient as this is a retrospective study. The interictal ECoG segment is sampled at either $512 \mathrm{~Hz}$ or $2048 \mathrm{~Hz}$ (see Table 3.1). An expert clinical neurophysiologist (FSSL) marked artefacts in the raw ECoG recordings, e.g. those arising from the reference electrode. In the next sections we explain how CC and GC networks are obtained from this data. Both methods require specific pre-processing steps. For example, for $\mathrm{CC}$ it is usual to band-filter the data, while for GC this is not recommended [100]. Also, it is common to apply differencing before calculating GC, while this is not the case for CC.

The protocol for SPES has been described in [40]. Specifically, ten monophasic electrical stimuli are applied to pairs of horizontally adjacent electrodes. The stimuli have a duration of $1 \mathrm{~ms}$ with an inter-stimulus time of $5 \mathrm{~s}$ and an intensity of $8 \mathrm{~mA}$. During SPES ECoG data has been registered at a sampling rate of $2048 \mathrm{~Hz}$.

For all selected patients, the ECoG grid consisted of one or two large grids, spatially arranged in four or six times eight electrodes, and some additional strips consisting of eight electrodes each. We discarded all data from electrodes not used to stimulate with SPES as well as dysfunctional electrodes. Table 3.1 shows the selected number of electrodes per patient.

\subsubsection{Cross-Correlation}

$\mathrm{CC}$ networks are non-directional weighted networks constructed from ongoing interictal ECoG data. For consistency, all ongoing ECoG data are resampled to $512 \mathrm{~Hz}$ if necessary. We band-pass filtered the data to the $\theta$-, $\alpha$ - and $\beta$-band, i.e. between 4 and $30 \mathrm{~Hz}$, following [101]. Next, we divided all segments of ECoG data without artefacts into non-overlapping epochs of 20 seconds (starting from the beginning of each segment and neglecting remaining parts or segments of less than 20 seconds). We selected the last 60 epochs, so 20 minutes in total, for further analysis. For each of the selected epochs we proceed as follows for every pair of electrodes. First, we estimate the cross-correlation function for all time lags $m$ with $|m| \leq M$ and $M$ the maximal lag in samples. Next, we set the connection strength as the 


\begin{tabular}{|l|l|l|l|l|l|}
\hline Pat & $f_{i i}$ & Grid configuration & $N_{e l}$ & $\mathrm{BW}$ & Patient state \\
\hline 1 & 2048 & $\mathrm{~F}(2 \times 8 ; 4 \times 8), \mathrm{IH}(1 \times 8)$ & 56 & $\mathrm{n}$ & Awake, agile \\
2 & 512 & $\mathrm{~F}(4 \times 8 ; 4 \times 8)$ & 56 & $\mathrm{n}$ & Awake, quiet \\
3 & 2048 & $\mathrm{~F}(4 \times 8), \mathrm{T}(4 \times 8), \mathrm{C}(1 \times 8), \mathrm{IH}(1 \times 8)$ & 72 & $\mathrm{y}$ & Light sleep \\
4 & 512 & $\mathrm{~T}(6 \times 8 ; 1 \times 8 ; 1 \times 8), \mathrm{F}(2 \times 8)$ & 58 & $\mathrm{n}$ & Light sleep \\
5 & 2048 & $\mathrm{~T}(2 \times 8), \mathrm{C}(4 \times 8)$ & 45 & $\mathrm{y}$ & Awake \\
6 & 512 & $\mathrm{~T}(6 \times 8 ; 2 \times 8 ; 1 \times 8 ; 1 \times 8), \mathrm{F}(2 \times 8)$ & 89 & $\mathrm{y}$ & Awake, language \\
& & & & & task \\
\hline
\end{tabular}

Table 3.1: Patient characteristics. $f_{i i}$ : sample frequency interictal EEG in $\mathrm{Hz}$, grid configuration: size and location (F: frontal, T: temporal, C: central, IH: inter-hemispheric) of the implanted electrodes, $N_{e l}$ : number of selected electrodes, BW: Broca's \& Wernicke's area covered by the grid (y: yes, n: no), Patient state: state of the patient during interictal recording.

maximum absolute value of this estimated cross-correlation function. We take a maximal lag of $M=26$ samples corresponding to a time of $50 \mathrm{~ms}$. We average over all 60 epochs to obtain the mean connectivity.

\subsubsection{Granger Causality}

GC networks are constructed from the same interictal ECoG data as CC networks. In contrast to $\mathrm{CC}$ networks, GC networks are directional. The main idea behind GC is that a connection from $x$ to $y$ is present if the prediction of the time series of $y$ improves significantly by incorporating the past of the time series of $x[93,102]$. In this study we use conditional GC, a multivariate form of GC, which besides the past of the time series $x$ and $y$ also uses the past of all other time series to determine the connectivity from $x$ to $y$. This method reduces spurious connectivity, e.g. connections that arise due to common input [103].

GC relies on fitting multivariate autoregressive models (MVAR models) to the data. The model order $m$ of this MVAR model determines the length of the history taken into account and must be specified. If we want to capture a history of $50 \mathrm{~ms}$ at a sampling rate of $512 \mathrm{~Hz}$, as in section 3.2.2, we would need $m=26$. For such high model orders many unknowns must be estimated in the MVAR model. To avoid overfitting of the model, enough data points and as a consequence long time series must be considered. For such long time series the assumption of (approximate) stationarity is likely to fail. By downsampling the required model order can be reduced, while a longer history can be taken into account [104, 105].

Our complete procedure to calculate GC is as follows. First, we resample the ECoG data to $128 \mathrm{~Hz}$. Next, first-order differencing is applied to enhance stationarity [106]. We select 60 epochs of 20 seconds in the same way as we do for CC (actually the same). Next, we calculate conditional GC in the time domain using the MVGC toolbox [103]. We set the model order to $m=7$, which is sufficient to capture $50 \mathrm{~ms}$ of history. Statistical significance is assessed using the recommended options of the MVGC toolbox, i.e. Granger's F-test with a significance level of 0.05 and the false discovery rate method to account for multi-hypothesis testing. For each epoch this results in a binary matrix with an entry being one if GC finds a significant connection and zero otherwise. Finally, we obtain the mean connectivity by averaging over all 60 epochs. The resulting network is directional with weights between zero and one. 


\subsubsection{SPES Network}

SPES networks are constructed using ERs. First, the ERs are detected from ECoG data using an automatic detector (see 3.S1). This detector determines whether an ER is present for every combination of stimulation pair and response electrode. Next, the SPES network is constructed. As for the CC and GC networks every node in this network represents an electrode. A connection from node $k$ to $l$ is present if at least one ER is detected at electrode $l$ after any stimulation involving electrode $k$. The resulting network is directional and has binary weights.

\subsubsection{Localizing Broca's and Wernicke's Area}

In three patients both the areas of Broca and Wernicke have been covered by the electrode grid. As part of clinical routine the precise locations of those two areas have been determined using electrocortical stimulation mapping (ESM). In ESM pulse trains of 4-7 seconds, $50 \mathrm{~Hz}$, 0.2-0.3 ms, 4-15 mA (stimulation amplitude was altered to avoid afterdischarges) are applied during a picture naming task and in case of Wernicke's area also during item presentation in a Token Test. If repeated stimulation interferes with language (either inability to name or understand, or paraphasia) and the cause is not anarthria (sound production is unaffected) the stimulated electrode pair is marked as positive for language. Stimulations are applied to horizontally and, in contrast to SPES, also vertically and diagonally adjacent electrode pairs. An individual electrode is marked positive if it was part of at least two positively marked pairs.

\subsubsection{Comparing Networks}

To compare CC and GC with SPES connectivity we need to cast the networks in the same form. We obtain binary CC and GC networks by thresholding the edge weights; if the weight of an edge exceeds this threshold, then there is a connection in the dichotomized network. The threshold $h^{*}$ is determined using a data-driven approach. This data-driven approach is inspired by both [107] and the definition of outliers in a boxplot. Let $Q_{1}$ and $Q_{3}$ denote the first and third quartile of the set of all edge weights. Then $Q_{3}-Q_{1}$ denotes the inter-quartile range, which is a measure for the spread. We set $h^{*}:=\max \left(Q_{3}+w\left(Q_{3}-Q_{1}\right), 0.1\right)$ with $w$ a parameter. We use $w=1.5$, which is the standard choice for defining outliers [107].

The dichotomized GC network and the SPES network are both directional, unweighted networks and hence they can be compared. A non-directional variant of the SPES network is constructed by putting an edge between nodes $i$ and $j$ if either $i \rightarrow j$ or $j \rightarrow i$ is present in the directional SPES network. This non-directional SPES network can be compared with the dichotomized CC network.

Next, we test if edges of the CC and GC networks coincide with those in the SPES network using a hypergeometric test for overrepresentation. Under the null hypothesis the connections of the functional network are distributed proportionally over existing and non-existing SPES connections. This hypothesis will be tested against the alternative hypothesis that $\mathrm{CC} / \mathrm{GC}$ connections are overrepresented in the set of SPES connections. In other words, we test whether it is more likely to find a CC/GC connection between two nodes if there is a SPES connection between these two nodes. 
The probability of finding $k \mathrm{CC} / \mathrm{GC}$ connections in a set of $n_{s}$ SPES connections (and consequently $n_{s}-k$ non-existing CC/GC connections) is, under the null hypothesis, given by a hypergeometric distribution:

$$
p_{n_{s}, n_{f}}(k)=\left(\begin{array}{c}
n_{f} \\
k
\end{array}\right)\left(\begin{array}{c}
n-n_{f} \\
n_{s}-k
\end{array}\right) /\left(\begin{array}{c}
n \\
n_{s}
\end{array}\right),
$$

with $n_{f}$ the total number of CC/GC connections and $n$ the total number of possible connections. We have $n=N_{e l}\left(N_{e l}-1\right)$ for the comparison between GC and SPES and $n=N_{e l}\left(N_{e l}-1\right) / 2$ for the comparison between CC and SPES. Let $n_{s f}$ denote the number of connections in both the SPES and the CC/GC network. Under the null hypothesis, the probability $P$ to have $n_{S f}$ or more $\mathrm{CC} / \mathrm{GC}$ connections in the set of SPES connections is given by:

$$
P=\sum_{k=n_{s f}}^{\min \left\{n_{s}, n_{f}\right\}} p_{n_{s}, n_{f}}(k) .
$$

We will reject the null hypothesis if $P<0.01$.

We also investigate the dependence of our results on the threshold for CC/GC. Let $h$ be the threshold for the $\mathrm{CC}$ or GC network. Take $a_{c}(h)$ as the fraction of positive agreement between the SPES and CC/GC network, i.e. the number of connections that arise in both the SPES and the CC/GC network dichotomized using threshold $h$ divided by the number of SPES connections. If $a_{c}$ is one all connections in the SPES network are also part of the CC/GC network. If $a_{c}$ is zero then none of the SPES connections are part of the CC/GC network. Equivalently, define $a_{n c}(h)$ as the fraction of negative agreement, i.e. the number of nonexisting SPES and CC/GC connections as a fraction of the total number of non-existing SPES connections. If $a_{n c}$ is one then all non-existing SPES connections are also non-existing in the $\mathrm{CC} / \mathrm{GC}$ network in which case all connections in the CC/GC network are part of the SPES network. Further, we calculate the total agreement, i.e. the number of agreeing connections and non-existing connections as fraction of the total number of possible connections. We define $h_{m a}$ as the threshold maximizing the total agreement.

Finally, we study connectivity between electrodes in Broca's and Wernicke's area in all three networks. We examine the number of connections found between both areas as a fraction of $n_{b w}$, the maximal number of possible connections between electrodes in Broca's and Wernicke's area. For the directional networks, i.e. SPES and GC, $n_{b w}$ is given by $2 n_{b} n_{w}$ and for the CC network by $n_{b} n_{w}$, where $n_{b}$ and $n_{w}$ denote the number of electrodes in Broca's and Wernicke's area respectively.

\subsection{Results}

\subsubsection{Cross-Correlation \& SPES}

In Figure 3.1B the agreement and disagreement between the adjacency matrices of the (nondirectionalized) SPES and (dichotomized) CC network for patient 2 is shown (see 3.S2 for others). Observe that $\mathrm{CC}$ connections are located mainly at the sub-diagonals of the connectivity matrix that are directly next to the diagonal or eight columns away from the diagonal. Physically these entries correspond to the nearest neighbours of an electrode. In 
contrast, SPES connections are also found between more distant nodes. Further, almost all of the CC connections are contained in the SPES network, while the reverse is not true. So, the strong CC connections form a subnetwork of the non-directionalized SPES network. Adding surrogate testing to quantify the significance of CC connections as in [108] yields the same results (results not shown).

The latter effect is robust with respect to changes in the threshold for the correlation network as indicated in the histogram in Figure 3.1C displaying the distribution of $\mathrm{CC}$ strengths. The distribution is somewhat skewed, with a peak around 0.1 and a long tail towards the higher correlation values. The peak consists mostly of pairs of nodes that are not connected in the SPES network, while the tail is almost completely constituted by SPES connections. If we therefore slightly change the threshold for the CC network, then the dichotomized CC network would still be contained almost entirely in the SPES network.

The latter is not the case anymore if the threshold is set to $h_{m a}$, for which the agreement between the networks is maximal (see Figure 3.1D)). As $h_{m a}$ is smaller than $h^{*}$ more connections are included in the $\mathrm{CC}$ network. The additional agreement comes at the expense of adding many more non-SPES connections to the CC network.

The observations above apply to all patients. In Table 3.2 the results of the statistics for overrepresentation are shown. In all six patients the $P$-values are small and hence CC connections are overrepresented in the SPES network. Figure 3.2A depicts the dependence on the threshold for CC in relation to the agreement with the SPES network. We observe that $a_{c}$ increases if the threshold of the CC network is lowered, while on the other hand $a_{n c}$ remains close to 1 for a relatively large range of thresholds. This observation means that for a broad range of thresholds the CC network is contained almost entirely in the SPES network. On the other hand, the network induced by CC contains between $20-45 \%$ of the connections of the SPES network for a negative agreement, $a_{n c}=0.95$. So the $\mathrm{CC}$ network forms only a part of the SPES network.

\begin{tabular}{|l|r|r|r|r|r|l|}
\hline Pat & $h^{*}$ & $n$ & $n_{s}$ & $n_{f}$ & $n_{s f}$ & $\mathrm{P}$ \\
\hline 1 & 0.44 & 1540 & 658 & 56 & 54 & $1.7 \cdot 10^{-18}$ \\
2 & 0.43 & 1540 & 566 & 134 & 128 & $7.0 \cdot 10^{-52}$ \\
3 & 0.23 & 2556 & 1535 & 283 & 246 & $1.3 \cdot 10^{-25}$ \\
4 & 0.55 & 1653 & 519 & 47 & 45 & $3.2 \cdot 10^{-21}$ \\
5 & 0.55 & 990 & 648 & 59 & 55 & $3.0 \cdot 10^{-7}$ \\
6 & 0.32 & 3916 & 2600 & 415 & 406 & $7.3 \cdot 10^{-64}$ \\
\hline
\end{tabular}

Table 3.2: Summary of statistics for comparison of CC and SPES.

\subsubsection{Granger Causality \& SPES}

Figure 3.3A shows the level of agreement between the adjacency matrices of the SPES and (dichotomized) GC network for patient 2 (see 3.S2 for others). Like CC connections, GC mainly finds connections between geometrically close nodes. Approximately $70 \%$ of the detected GC connections are part of the SPES network. A histogram containing the distribution of GC strengths is displayed in Figure 3.3B. This distribution has its maximum at 0 and decays quickly. The thin tail of the distribution is mostly constituted by SPES 
A

\begin{tabular}{|c|c|c|c|c|c|c|c|}
\hline & & 1 & 2 & 3 & 4 & 5 & 6 \\
\hline & 7 & 8 & 9 & 10 & 11 & 12 & 13 \\
\hline \multirow[t]{2}{*}{14} & 15 & 16 & 17 & 18 & 19 & 20 & 21 \\
\hline & 22 & 23 & 24 & & 25 & 26 & 27 \\
\hline \multirow[t]{2}{*}{28} & 29 & 30 & 31 & 32 & 33 & 34 & 35 \\
\hline & 36 & 37 & 38 & 39 & 40 & 41 & 42 \\
\hline 43 & 44 & 45 & 46 & 47 & 48 & 49 & \\
\hline 50 & 51 & 52 & 53 & & 54 & 55 & 56 \\
\hline
\end{tabular}

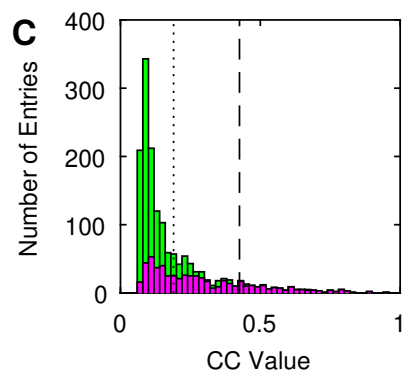

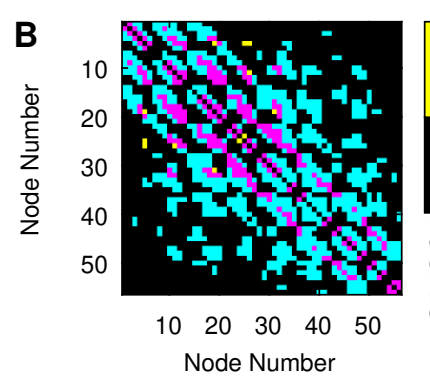
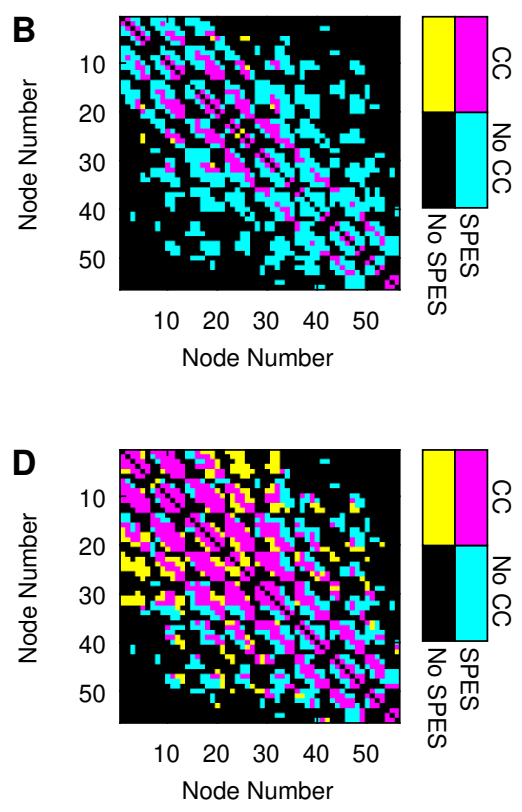

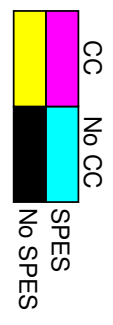$$
\text { . }
$$

Figure 3.1: Patient 2 (A) Schematic layout of the electrode grid. (B, D) Comparison of the adjacency matrices of the SPES and CC network for threshold $h^{*}$ and $h_{m a}$ respectively. The numbers of the electrodes correspond to the layout in A. (C) Histogram of the distribution of the CC connections. The dashed and dotted lines indicate the thresholds $h^{*}$ and $h_{m a}$ respectively.
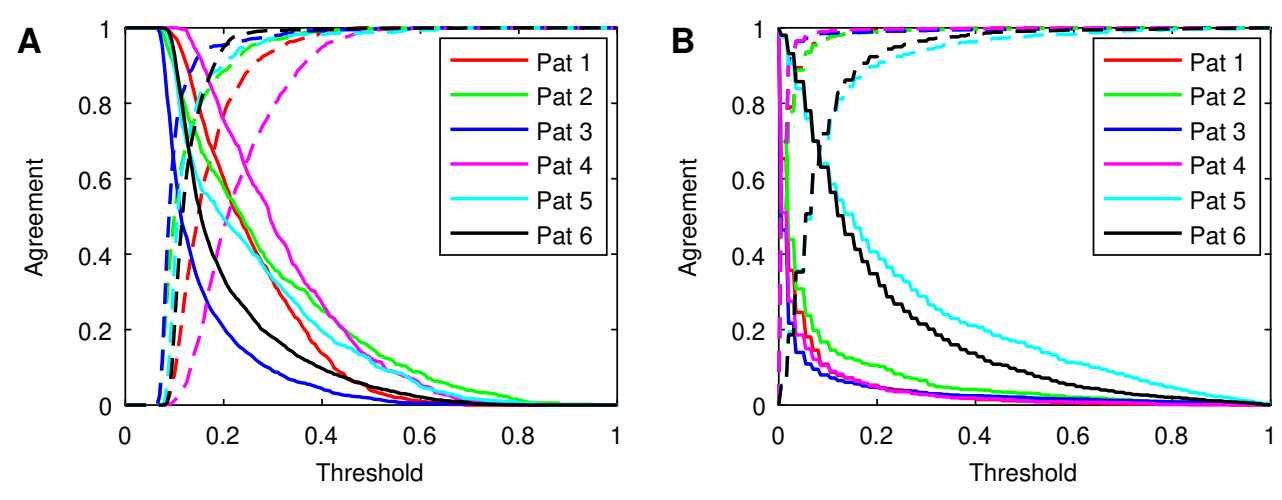

Figure 3.2: $a_{c}$ (solid) and $a_{n c}$ (dashed) as function of the threshold for (A) SPES \& CC and (B) SPES $\& \mathrm{GC}$ for all patients

connections.

The observations above apply to all patients and suggest that GC connections are overrepresented in the SPES network. It follows from the statistical test that this overrepresentation is indeed the case as can be seen in Table 3.3. The dependence on the threshold for GC in relation to the agreement with the SPES network can be found in Figure 3.2B. Although 
the effect is weaker than in the CC case, $a_{c}$ increases if the threshold of the $\mathrm{CC}$ network is lowered, while $a_{n c}$ remains close to one for a relatively large range of thresholds. For patient 1 approximately $12 \%$ of the SPES connections are part of the GC network for $a_{n c}=0.95$, in the other patients this is much higher and varies between $20-25 \%$.
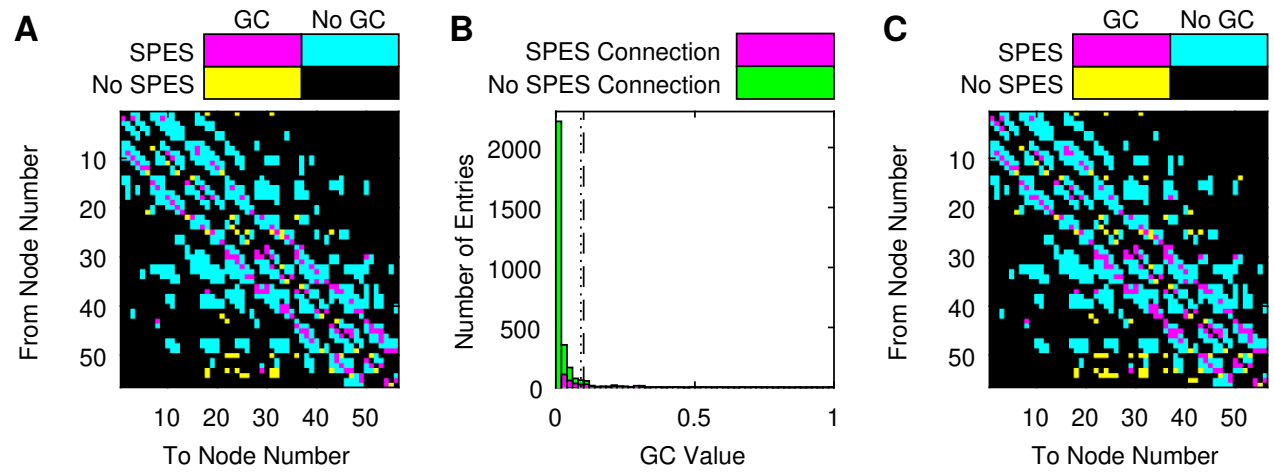

Figure 3.3: Patient $2(\mathbf{A}, \mathbf{C})$ Comparison of the adjacency matrices of the SPES and GC network for thresholds $h^{*}$ and $h_{m a}$, respectively. The numbers of the electrodes correspond to the layout in Figure 3.1A. (B) Histogram of the distribution of the GC connections. The dashed and dotted lines indicate the thresholds $h^{*}$ and $h_{m a}$ respectively.

\begin{tabular}{|l|r|r|r|r|r|l|}
\hline Pat & $h^{*}$ & $n$ & $n_{s}$ & $n_{f}$ & $n_{s f}$ & $\mathrm{P}$ \\
\hline 1 & 0.10 & 3080 & 969 & 165 & 105 & $2.3 \cdot 10^{-18}$ \\
2 & 0.10 & 3080 & 825 & 205 & 138 & $2.5 \cdot 10^{-36}$ \\
3 & 0.10 & 5112 & 2193 & 219 & 175 & $2.6 \cdot 10^{-30}$ \\
4 & 0.10 & 3306 & 791 & 107 & 83 & $1.3 \cdot 10^{-32}$ \\
5 & 0.45 & 1980 & 980 & 209 & 181 & $1.6 \cdot 10^{-32}$ \\
6 & 0.37 & 7832 & 3739 & 636 & 550 & $9.1 \cdot 10^{-101}$ \\
\hline
\end{tabular}

Table 3.3: Summary of statistics for comparison of GC and SPES.

\subsubsection{Broca-Wernicke Connectivity}

The electrode grids of patients 3, 5 and 6 covered both Broca's and Wernicke's area. In Figures 3.4, 3.5 and 3.6 the SPES, CC and GC networks restricted to the electrodes in these areas are displayed for these patients. For patient 3 Broca's area consists of four electrodes. Three of them $(50,51$ and 58) are neighbouring electrodes, while 34 is located further away, i.e. $2 \mathrm{~cm}$ away from 50 (see 3.S2 for the schematic location of the electrodes). Wernicke's area is covered by two neighbouring electrodes. In Broca's area $67 \%$ of all possible connections are found in the SPES network, while for CC and GC this is 50\% and 17\% respectively. In both the $\mathrm{CC}$ and GC network electrode 34 is isolated, which agrees with our previous finding that those methods find predominantly close-by connections. SPES, however, finds connections to this more distant node. On the other hand, electrodes 50 and 51 are reciprocally connected 
in both the CC and GC network, while there is no edge in the SPES network between those two electrodes. The absence of SPES connections is partly a consequence of how we build the SPES network. As electrode 51 is located at the end of a row in the grid it is part of only one stimulation pair, namely 50-51, therefore it is impossible to find a connection from 51 to 50 in the SPES network. The reverse connection was also not found as electrode 51 became saturated when stimulating electrode pair 49-50. The two electrodes in Wernicke's area are reciprocally connected in the CC and GC network, while SPES could not recover the connection from 31 to 32 as the only stimulation pair containing electrode 31 is the pair 31-32. Connectivity between Broca's and Wernicke's areas is only found in the SPES network. All the connections except one are from Broca's to Wernicke's area.
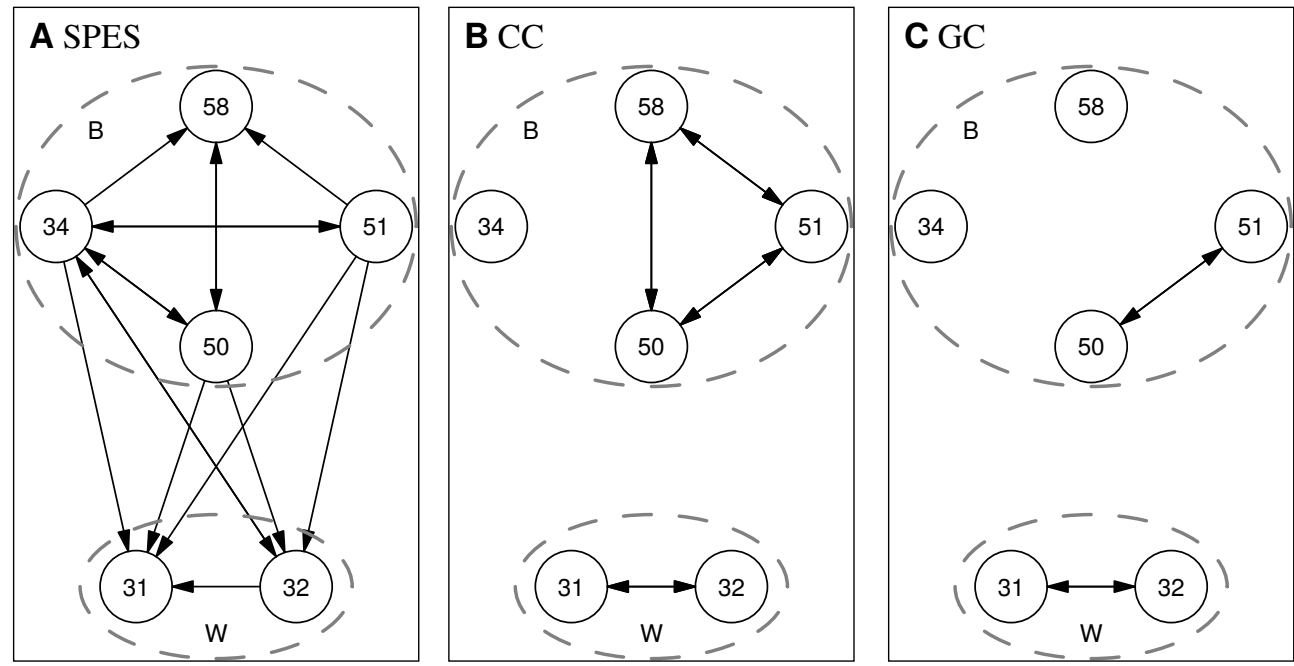

Figure 3.4: Connectivity between nodes in Broca (B) and Wernicke (W) for patient 3 inferred by (A) SPES, (B) CC and (C) GC. CC and GC networks are dichotomized using threshold $h^{*}$.

For patient 5, Broca's and Wernicke's areas consist of 3 and 4 neighbouring electrodes respectively (see 3.S2 for the location of the electrodes). The electrodes in Broca's area are fully connected to each other in the SPES network. In the CC and GC networks we find only a third of all possible connections in Broca. In Wernicke's area the SPES network is also well-connected, containing $75 \%$ of all possible connections. The $\mathrm{CC}$ network has a relatively high internal connectivity, as $2 / 3$ of all possible connections are present. In the GC network on the other hand, only a quarter of all possible connections are found. For patient 5, as for patient 3, only the SPES network shows connectivity between Broca's and Wernicke's area, however the orientation is reversed; all connections except one are directed from Wernicke's to Broca's area.

For the last patient, 2 electrodes were placed on Broca's area while there were 6 on Wernicke's area. In all three networks the 2 electrodes in Broca's area were reciprocally connected. In Wernicke's area the SPES network contains $93 \%$ of all possible connections. The CC and GC are also relatively well-connected finding $80 \%$ and a half of all possible connections respectively. In contrast to the other two patients some $\mathrm{CC}$ and $\mathrm{GC}$ connections are found between Broca's and Wernicke's area. For GC this is the reciprocal connection 

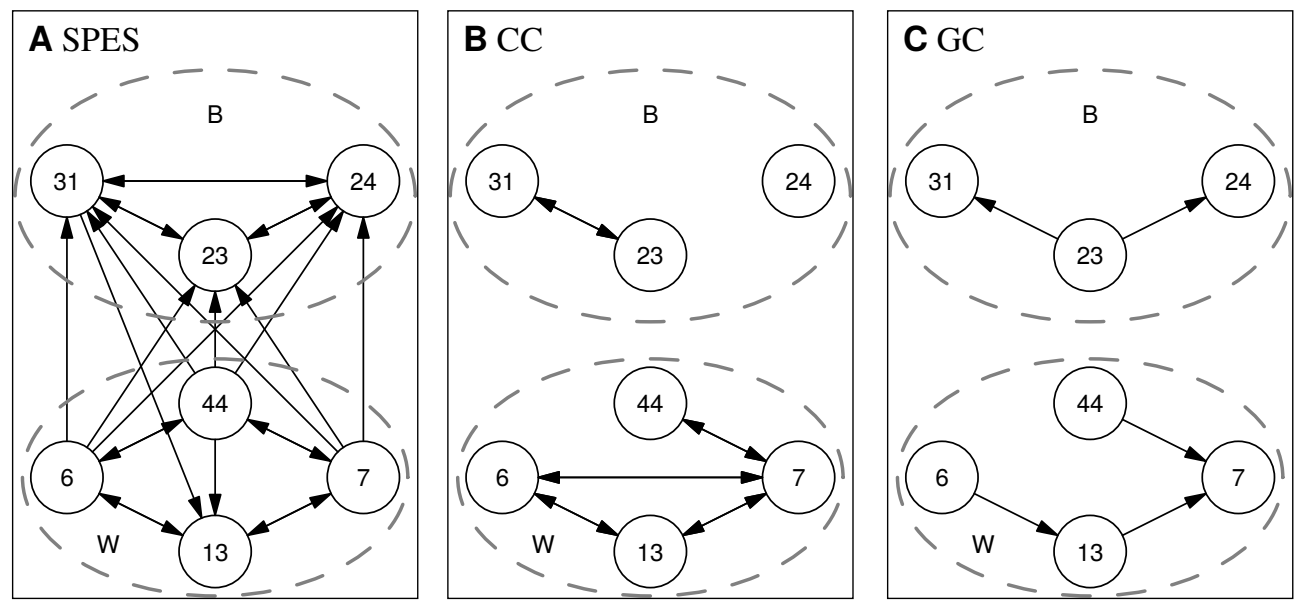

Figure 3.5: Connectivity between nodes in Broca (B) and Wernicke (W) for patient 5 inferred by (A) SPES, (B) CC and (C) GC. CC and GC networks are dichotomized using threshold $h^{*}$.

between electrodes 10 and 30, which is $8 \%$ of all possible connections. The CC network also contains a connection between 9 and 30, yielding a total of $17 \%$ of all possible connections. These percentages are still much lower than the SPES network which finds one third of all possible connections from Broca's area to Wernicke's area and one half in opposite direction.
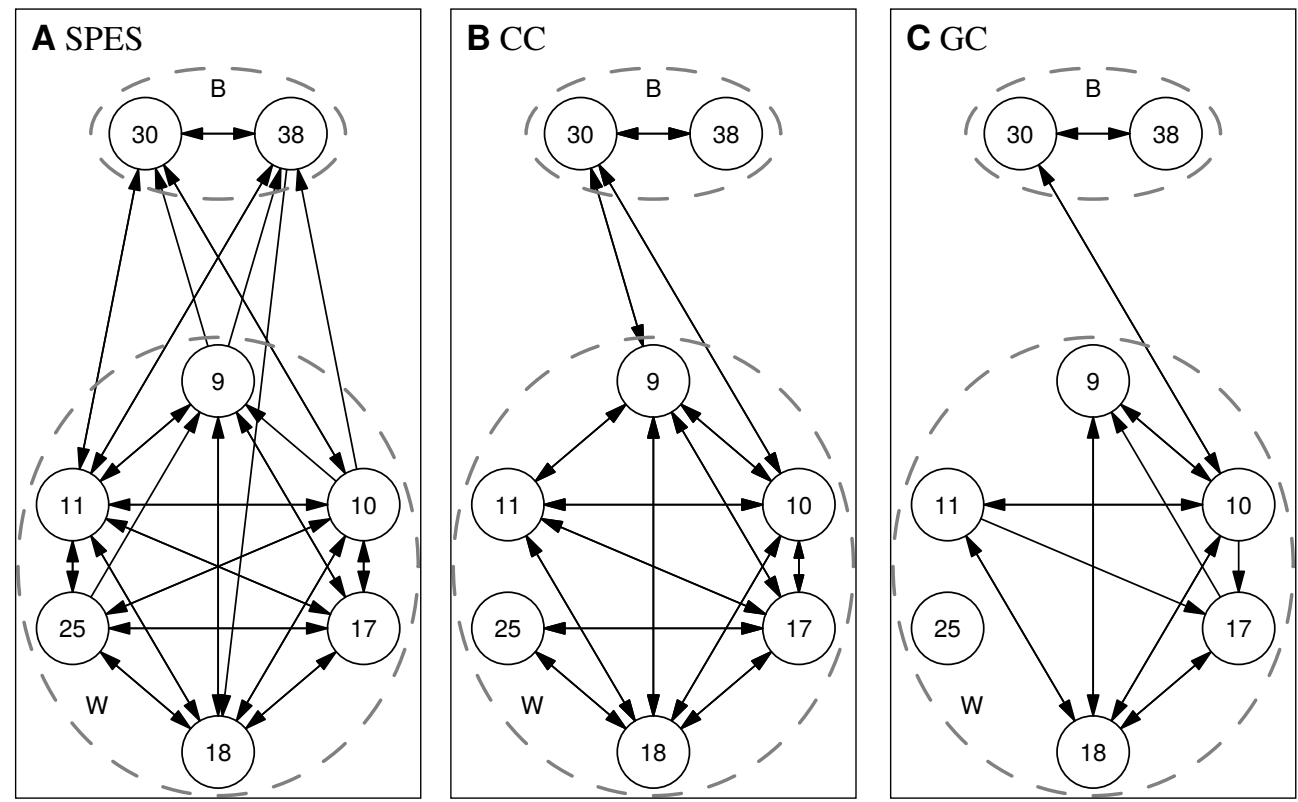

Figure 3.6: Connectivity between nodes in Broca (B) and Wernicke (W) for patient 6 inferred by (A) SPES, (B) CC and (C) GC. CC and GC networks are dichotomized using threshold $h^{*}$. 


\subsection{Discussion}

\subsubsection{Network Comparison}

We have compared connectivity derived from SPES to CC and GC networks derived from ongoing interictal ECoG. All three methods yield primarily nearest neighbour connections, however SPES networks are usually connected more densely and include more distant connections than $\mathrm{CC}$ and GC networks. We find a strong relationship between CC and SPES networks, i.e. strong CC connections form a subset of the SPES network. The relation between SPES and GC networks is weaker. Although GC connections coincide more frequently with SPES connections compared to non-existing SPES connections, they do not form a subset of the SPES network.

One of the most important factors underlying the difference between the SPES and CC/GC networks is the process by which networks are inferred. Non-evoked connectivity methods try to recover connectivity from passive observations using statistical dependencies between the time series of nodes. Long range connections may have small ongoing influences that are easily overpowered by activity of local circuits and their common input. CC and GC will therefore find only weak coupling between such nodes, as has been observed in a computational modeling study [109]. This phenomenon might be typical for the centimeter-scale at which ECoG is recorded. In SPES, connections are activated strongly, due to the electrical stimulus. This causes a large response at the receiving electrode, which makes it distinguishable from the ongoing activity. This might explain the difficulty of detecting long-range connections among networks based on ongoing ECoG activity, compared to SPES networks.

In our study, all three constructed networks use the electrodes of the ECoG grid as nodes which enables a straightforward comparison between the networks. Moreover, as all three networks represent local connectivity their scale is equal. Other studies have compared SPES to larger scale networks inferred using whole-brain imaging modalities like diffusion weighted imaging (DWI) and resting-state functional magnetic resonance imaging (fMRI). A higher overlap than expected by chance was found between edges of dichotomized DWI and SPES networks [99]. On the other hand, the correlation between connection strengths taken over the whole brain is low both for comparing SPES with DWI [98, 99, 110] and fMRI [110, 111]. There are however clusters of brain regions that have similar connectivity in networks constructed with SPES as well as DWI [98] and fMRI [111].

An example of such a cluster is the language circuit, in which a strong relation was found between DWI and SPES amplitude and latency [97]. This relation is in accordance with earlier studies on DWI [112] and CCEP [113] in the language circuit. Similar results were found for a comparison between the amplitude of CCEP and resting-state fMRI [111]. Here it was found that CCEP connections starting from Broca, Wernicke or sensory-motor regions show a much higher resting-state fMRI connectivity compared to non-existing CCEP connections starting from these regions.

The classical language circuit was the first functional network studied with CCEP [113]. Bidirectional connectivity between Broca's and Wernicke's area was found, in contrast to traditional Wernicke-Geschwind model, which hypothesized only connectivity from Wernicke's to Broca's area [114]. Stimulation of electrodes in Wernicke's area elicits less well-pronounced responses in electrodes in Broca's area compared to the other way around. These results have been confirmed by multiple CCEP studies (see [34] for an overview). Our findings are 
in accordance with these results. However, in patient 3 the connections are predominantly from Broca's to Wernicke's area, while in patient 5 the reverse holds. This result could be a consequence of our definition of Broca's and Wernicke's area, namely as electrodes that are part of at least two stimulation pairs marked positive for language using ESM.

In contrast to the SPES network, we find that $\mathrm{CC}$ and $\mathrm{GC}$ networks reveal only some connectivity between Broca's and Wernicke's area in one of the three patients. This result could be expected because those two methods yield mostly local connections. Another explanation could be the state of the patient during ECoG acquisition. The only patient for which CC and GC reveal connections between Broca and Wernicke, patient 6, was busy with a language task during the ongoing ECoG recordings. One could therefore expect that Broca's and Wernicke's area were more active. Nevertheless, CC and GC find only a fraction of the connections that are found with SPES.

\subsubsection{Methodological Issues}

There are many methodological issues in constructing functional networks from ongoing ECoG starting already with pre-processing of the ECoG data. First of all, there are multiple options for referencing ECoG data. We used a common reference montage with an extracranial reference electrode located on the contralateral mastoid. A common reference montage has the disadvantage that noise on the reference electrode affects all channels of the recorded ECoG data. For an extracranial reference this is more problematic than for an intracranial reference, as the former is more susceptible to pick up muscle artefacts. Unfortunately, it was not possible to use an intracranial reference electrode as we do not apply epidural reference electrodes during grid recordings. To reduce the influence of common reference noise we removed the parts of the recordings in which common reference artefacts were visible according to an expert clinical neurophysiologist (FSSL). Further, the common reference noise will increase the level of background correlations which may lead to spurious connections [115]. We therefore used the data-driven threshold $h^{*}$ which ensures that only correlations that are sufficiently above the background level will be included, at the cost of a less extended dichotomized network. As an alternative to a common reference montage bipolar and average reference montages may be used. These montages try to remove the common reference noise by taking linear combinations of the signals, but this changes the interpretation of the nodes and, moreover, it creates linear dependencies between the signals. The latter proved to be problematic when we tried to calculate multivariate GC. Given these practical constraints, this is the only approach allowing us to compare the networks.

Another methodological issue is the length of the epochs for calculating functional connectivity. If those epochs are too long the time series might not be stationary. On the other hand, the epochs should be long enough to reliably infer the connectivity. In the case of $\mathrm{CC}$ for example, two finite independent time series may show a high correlation, although this is theoretically zero for infinite ones [107]. In this study we took epochs of twenty seconds as preliminary investigations showed the $\mathrm{CC}$ values to stabilize for longer epochs. For consistency we used the same epoch length for GC.

We observed that $\mathrm{CC}$ is a robust functional connectivity measure. GC in contrast, is much more sensitive to non-stationarity of the time series and small artefacts. This sensitivity might be because GC is a noise-driven method which needs a certain amount of stochasticity in the data. In ECoG recordings this stochasticity can be too small causing the factors mentioned 
above to dominate the time series [103].

The SPES networks we constructed are based on ERs found with our automatic detector. The principle of the detector is straightforward and uses the amplitude of responses relative to the baseline to qualify a response as ER or not. Similar principles are applied in other studies $[32,96,116]$. We have validated our detector on visually classified responses (see 3.S1). An alternative for our binary classification is to use the amplitude of the response itself to infer the strength of a connection as is often used in CCEP studies [35, 97, 113]. The amplitude, however, depends on multiple factors including how well an electrode makes contact to the cortex. Alternatively, a variable amplitude protocol may be used to infer connection strengths for SPES [95, 98].

Another potential problem in the construction of SPES networks is the effect of volume conduction (VC) [117]. Due to the direct and artificial nature of the stimulation, a large source of neuronal activity can be generated which might be picked up by electrodes surrounding the stimulation pair. Note that this phenomenon is not about VC of the electrical stimulation itself. The result could be that spurious local connectivity is found [117]. We investigated the influence of VC in 3.S3 and concluded that its effects in our SPES data are small. It is, therefore, not necessary to account for VC effects in our SPES networks.

A point of attention is the non-stationarity of brain connectivity. We chose to select ongoing ECoG data recorded just preceding SPES as the patient state during that time would be most similar to the one during stimulation. Although functional connectivity was calculated over several minutes, we noted that differences in the results were small in general. The network converged rapidly to an average structure as has been described before [118]. In contrast to functional connectivity, the presence of a SPES connection is measured over approximately one minute. One might therefore think that variation in SPES connectivity is high, however this is not the case; SPES networks are highly reproducible. In preliminary research we found an agreement in connectivity between two SPES sessions of around 75\%, which is in-line with another CCEP study [96].

\subsubsection{Overview}

The data in this study is obtained from intracranial grid recordings in patients with refractory epilepsy. In this field the study of brain networks may help to improve localization of the epiletogenic focus [72, 87]. An interesting recent development is to combine networks with computational models [Chap. 2, 49, 76]. In these models, the activity of the neuronal population underlying each node is modeled by a neural mass. The nodes influence each others' dynamics according to the connectivity of the network. By using patient-specific networks the effect of epilepsy surgery can be predicted [65-67, 119], however many challenges still remain before these methods could be applied in clinical practice [120,121].

One of the questions in this approach is which type of network should be used. To date, computational studies have used networks derived from ongoing intracranial EEG data, both interictal [66] as well as seizure data [65, 119]. However, as we showed in this study, interictal $\mathrm{CC}$ and GC networks do not capture all known anatomical connections, for instance those between Broca and Wernicke. SPES networks might be a good alternative as such models would incorporate more physiological connections. Moreover, SPES ER networks contain information about both the seizure onset zone $[37,38]$ and seizure propagation $[35,36]$. It would therefore be interesting to compare SPES and ictal networks. 
Another issue is the limited covering of electrode grids which results in localized sampling only. In a computational network approach this limited covering leads to boundary effects. A possible solution is to incorporate a patient specific-network into a larger, generic, full-brain network. For SPES, there are some brain atlases available $[98,116]$ which could be beneficial for such studies. Seen in this light our work gives a better foundation for the use of networks in such future computational studies.

\section{S1 Automatic Detection of ERs}

In this supplement we provide a detailed description of our automatic detector (AD) for ERs. First, the detector algorithm is explained. Next, we describe our gold standard for ER detection, i.e. visual classification. Finally, we use a learning set to tune the parameters of the $\mathrm{AD}$ and test the AD's performance on a validation set.

\section{S1.1 Automatic Detector Algorithm}

The basic principle of the AD is that an ER yields a local extremum within $100 \mathrm{~ms}$ after the stimulation that is "sufficiently" high above baseline. The input for the AD is ECoG data recorded during SPES. In our SPES protocol each stimulation pair is probed ten times with an inter-stimulus time of 5 seconds. Around each stimulus an epoch of 5 seconds starting $2 \mathrm{~s}$ before the stimulus is selected. We average all ten epochs and subtract the median taken over the whole interval of the averaged response.

Next, we detect the extrema in a time range of $9 \mathrm{~ms}$ to $100 \mathrm{~ms}$ after the stimulation using the Matlab function peakfinder. This function has a parameter $s$, specifying how much an extreme value should deviate from the neighbouring time points to qualify as an extremum. Let $\sigma_{b l}$ be the standard deviation of the 2 seconds prior to stimulation. We set $\sigma=\max \left\{\sigma_{b l}, \sigma_{\min }\right\}$, where $\sigma_{\min }$ is a user-defined parameter. If the amplitude of the extreme value is higher than a threshold $\theta \sigma$, then the AD classifies the response as an ER. This procedure is illustrated in Figure 3.7B and C.

\section{S1.2 Performance of the Automatic Detector}

We use visual classification of ERs as gold standard for ER detection. ERs were annotated in Micromed, SystemPlus Evolution by one observer (DvB). ECoG data was visualized with $5 \mathrm{~s} /$ page and a variable scaling (usually $1200 \mu \mathrm{V} / \mathrm{cm}$ ), depending on the amplitude of the signals and the number of implanted electrodes. If amplitudes were too high to be able to differentiate ERs in neighboring electrodes, the scaling was decreased to $2000 \mu \mathrm{V} / \mathrm{cm}$. No additional software filtering was used. Figure 3.7A shows some examples of visually classified ERs. In total we have annotated data of six patients, which we equally divide into a learning and validation set. The SPES data of two patients is also used in the main part of this work, i.e. patients A3 and A4 correspond to patients 3 and 4, respectively.

The data of the learning set is used to tune the three parameters of the AD: $s, \sigma_{\min }$ and $\theta$. We vary $s$ between 10 and $200 \mu \mathrm{V}$ with steps of $10 \mu \mathrm{V}, \sigma_{\min }$ between 0 and $100 \mu \mathrm{V}$ with steps of $10 \mu \mathrm{V}$ and $\theta$ between 1 and 15 with step size 0.5 . For each parameter combination we determine the true positives (visually annotated ERs detected by the AD) and the true negatives 
A

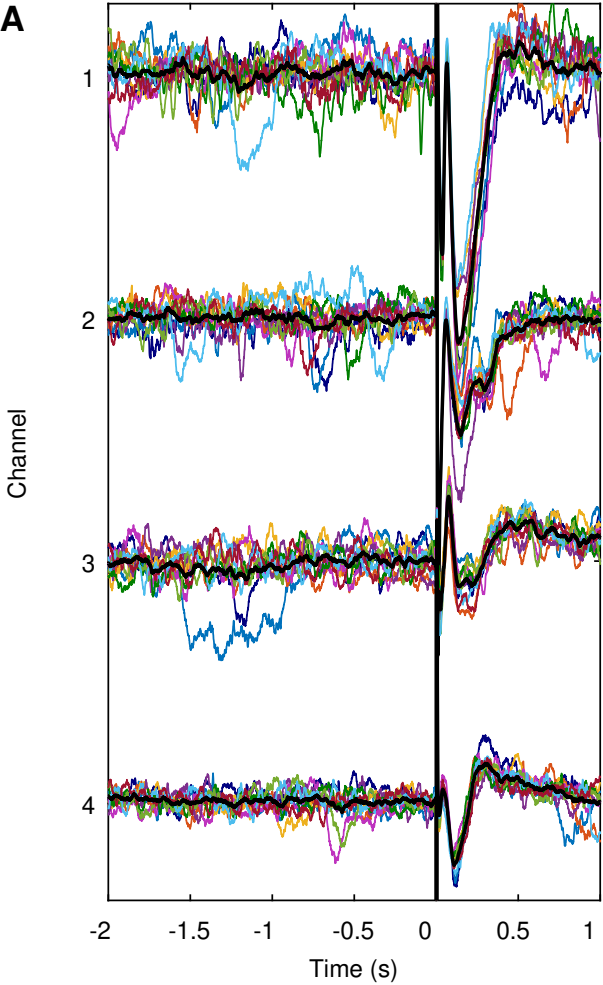

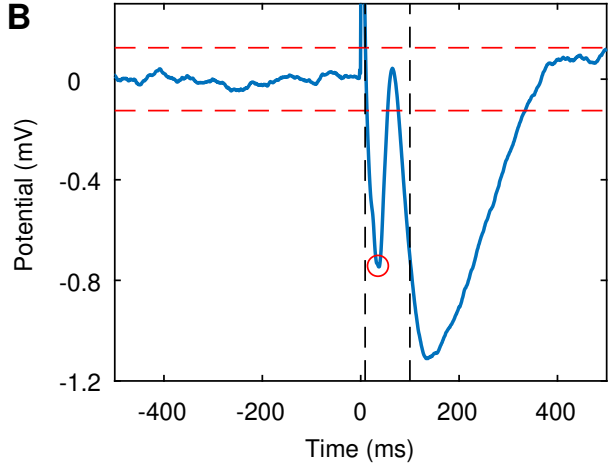

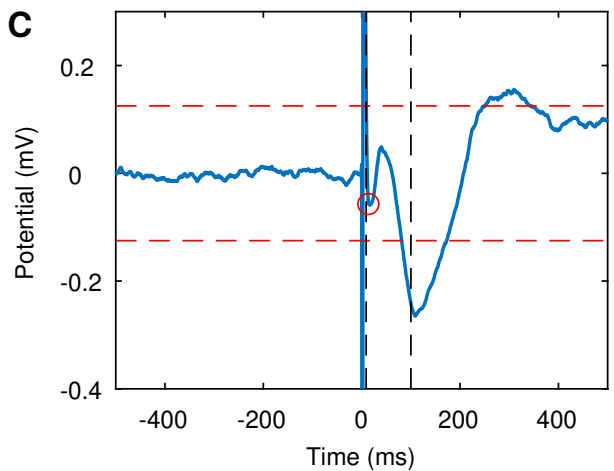

Figure 3.7: (A) Examples of visually classified ERs. The thin coloured lines indicate the responses to single stimulation trials, while the thick black lines indicate the averaged responses over all ten trials. The responses for channels 1, 2 and 3 were also classified as an ER by the automatic detector. (B, C) The blue line shows the magnification of the average response around the stimulation of channels 1 and 4 respectively. The dashed red lines indicate $\pm \theta \sigma$, the threshold for detecting an ER. A response is classified as ER if the amplitude of the detected peak exceeds the threshold in the time interval between the dashed black lines which is the case for $\mathbf{B}$ but not for $\mathbf{C}$.

(responses not classified as ER by both visual annotation and AD). From this we calculate the true positive rate, $t p r$, as the number of true positives divided by the total number of visually annotated ERs and the true negative rate, $t n r$, as the number of true negatives divided by the total number of responses not classified as ER by visual detection. In case of an ideal detector both $t p r$ and $t n r$ will be one. For each parameter combination we measure the quality of the AD by the distance $d$ of $t p r$ and $t n r$ to the perfect detection:

$$
d(t p r, t n r)=\sqrt{(1-t p r)^{2}+(1-t n r)^{2}} .
$$

We minimize $d$ to find the optimal parameter values for the AD. The optimal parameters are a threshold $\theta$ of 2.5,s $=20 \mu \mathrm{V}$ and a minimal standard deviation $\sigma_{\min }$ of $50 \mu \mathrm{V}$. For these optimal parameters, we have $d=0.23$. The performance on the learning set can be found in Table 3.4. This table shows, besides $t p r$ and $t n r$, also the positive predicted value 
( $p p v$, number of true positives divided by total number of automatic detected ERs), negative predicted value ( $n p v$, number of true negative divided by total number of responses not classified as ER by automatic detection) and accuracy (acc, fraction of correctly identified responses by the $\mathrm{AD})$.

We evaluate the automatic detector with the optimal parameter settings on the validation set. Also these results can be found in Table 3.4. The mean sensitivity and specificity are both high and vary within an acceptable range. So for each patient most of the responses classified as ER by the AD are also annotated as ERs by the visual detection and only a few of the visually annotated ERs are missed by the detector. We conclude that the performance is sufficient to construct a physiological network that is reliable for further analysis.

\begin{tabular}{|c|c|c|c|c|c|c|}
\hline Set & Patient & $t p r$ & $t n r$ & $p p v$ & $n p v$ & $a c c$ \\
\hline \multirow{4}{*}{ 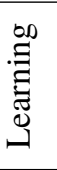 } & A1 & 0.85 & 0.83 & 0.57 & 0.96 & 0.83 \\
\hline & A2 & 0.73 & 0.88 & 0.60 & 0.93 & 0.85 \\
\hline & A3 & 0.83 & 0.79 & 0.44 & 0.96 & 0.79 \\
\hline & mean & 0.80 & 0.83 & 0.54 & 0.95 & 0.83 \\
\hline \multirow{4}{*}{ 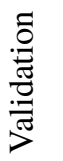 } & A4 & 0.71 & 0.94 & 0.84 & 0.87 & 0.86 \\
\hline & A5 & 0.77 & 0.94 & 0.68 & 0.96 & 0.92 \\
\hline & A6 & 0.86 & 0.85 & 0.72 & 0.93 & 0.85 \\
\hline & mean & 0.78 & 0.91 & 0.75 & 0.92 & 0.88 \\
\hline
\end{tabular}

Table 3.4: Performance of the automatic ER detector.

\section{S2 Detailed Results of Other Patients}

In this supplement we show detailed results of comparing SPES to cross-correlation (CC) and Granger Causality (GC) for patients 1 and 3-6. Figures 3.8-3.12 show for each patient the schematic layout of the electrode configuration as well as the comparison between SPES and CC and SPES and GC in the same way as Figures 3.1 and 3.3. The results in these patients are similar to those of patient 2 , which are presented in the main part of our work.

\section{S3 SPES \& Volume Conduction}

Volume conduction (VC) may play a role in the observation of evoked responses by single pulse electrical stimulation (SPES). Due to its direct and artificial nature, SPES can evoke a large source of neuronal activity which might be picked up by multiple nearby electrodes. In this supplement we investigate this effect by looking at the timing of the N1 peak of an early response (ER, see Figure 3.13). The timing of the N1 across electrodes will be exactly the same if ERs arise due to VC, as this acts instantaneously.

\section{S3.1 Detection of N1 Peaks}

We take, for the same patient population as used in the main text, the ECoG data of all ERs found by our automatic detector (see 3.S1). For each ER we try to find its three characteristic 
A

\begin{tabular}{|l|l|l|l|l|l|l|l|}
\hline 56 & 55 & 54 & 53 & 52 & 51 & 50 & 49 \\
\hline
\end{tabular}

\begin{tabular}{|c|c|c|c|c|c|c|c|c|}
\hline & 1 & 2 & 3 & 4 & 5 & 6 & 7 & 8 \\
\hline & 9 & 10 & 11 & 12 & 13 & 14 & 15 & 16 \\
\hline 17 & 18 & 19 & 20 & 21 & 22 & 23 & 24 & \\
\hline 25 & 26 & 27 & 28 & 29 & 30 & 31 & 32 & \\
\hline 33 & 34 & 35 & 36 & 37 & 38 & 39 & 40 & \\
\hline 41 & 42 & 43 & 44 & 45 & 46 & 47 & 48 & \\
\hline
\end{tabular}

B
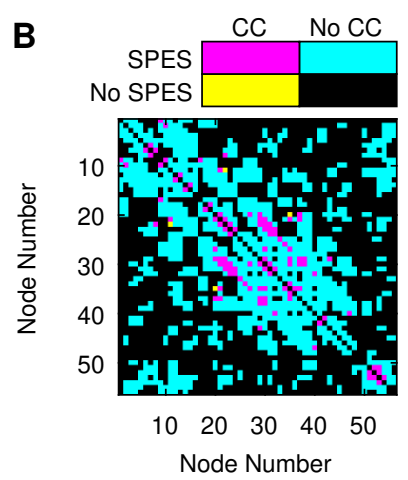

E
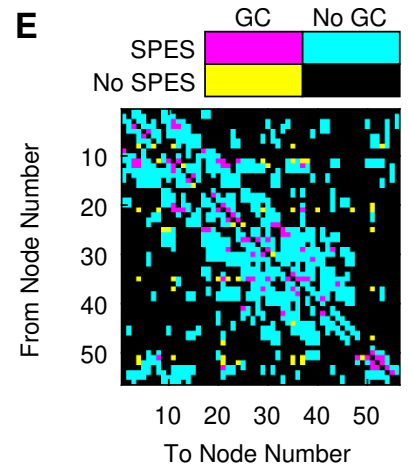

C

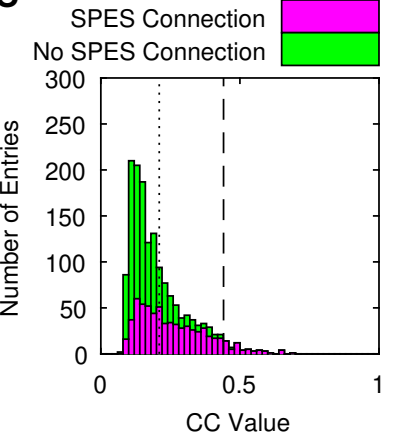

$\mathbf{F}$

SPES Connection No SPES Connection

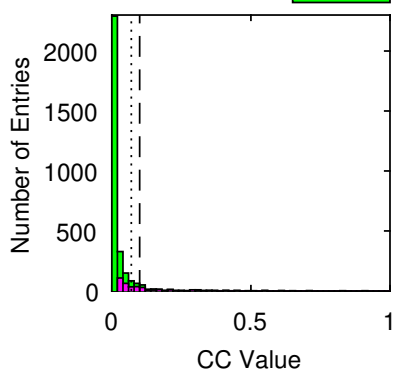

D
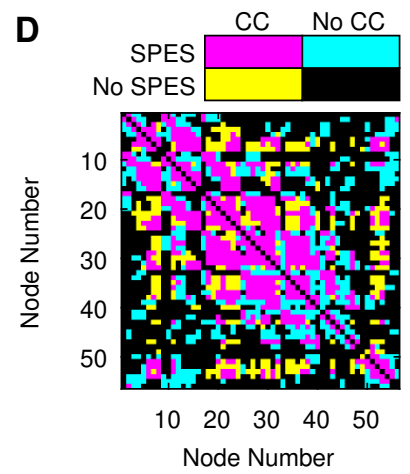

G
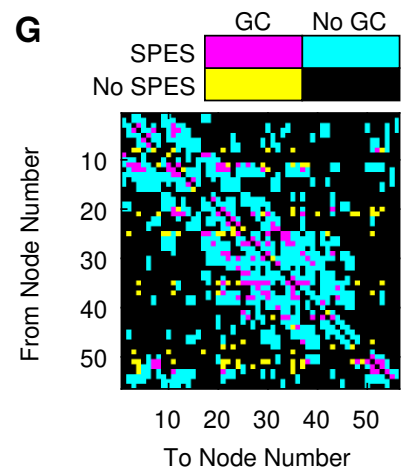

Figure 3.8: Results for patient 1. 


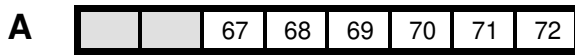

\begin{tabular}{|c|c|c|c|c|c|c|c|c|c|}
\hline & & & & 1 & 2 & 3 & 4 & 5 & \\
\hline & 6 & 7 & 8 & 9 & 10 & 11 & 12 & 13 & \\
\hline & 14 & 15 & 16 & 17 & 18 & 19 & 20 & 21 & \\
\hline & & 22 & 23 & 24 & 25 & 26 & 27 & 28 & 66 \\
\hline \multirow{8}{*}{\multicolumn{2}{|c|}{$\begin{array}{l}\text { Excluded Electrode } \\
\text { Broca's Area } \\
\text { Wernicke's Area }\end{array}$}} & & & & 35 & 43 & 51 & & 65 \\
\hline & & & & & 34 & 42 & 50 & 58 & 64 \\
\hline & & & & & 33 & 41 & 49 & 57 & 63 \\
\hline & & & & & 32 & 40 & 48 & 56 & 62 \\
\hline & & & & & 31 & 39 & 47 & 55 & 61 \\
\hline & & & & & & 38 & 46 & 54 & 60 \\
\hline & & & & & 30 & 37 & 45 & 53 & 59 \\
\hline & & & & & 29 & 36 & 44 & 52 & \\
\hline
\end{tabular}
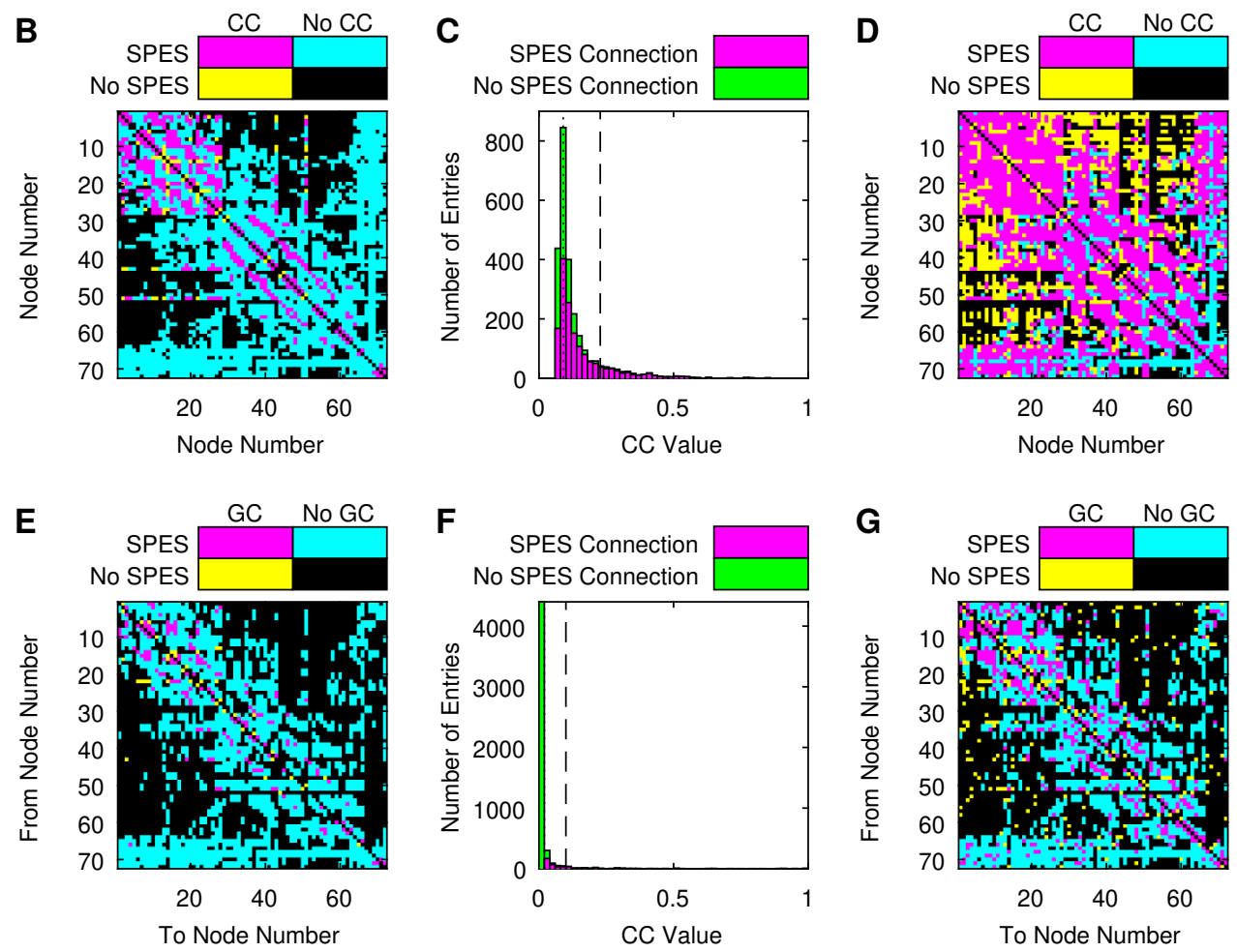

Figure 3.9: Results for patient 3. 
A

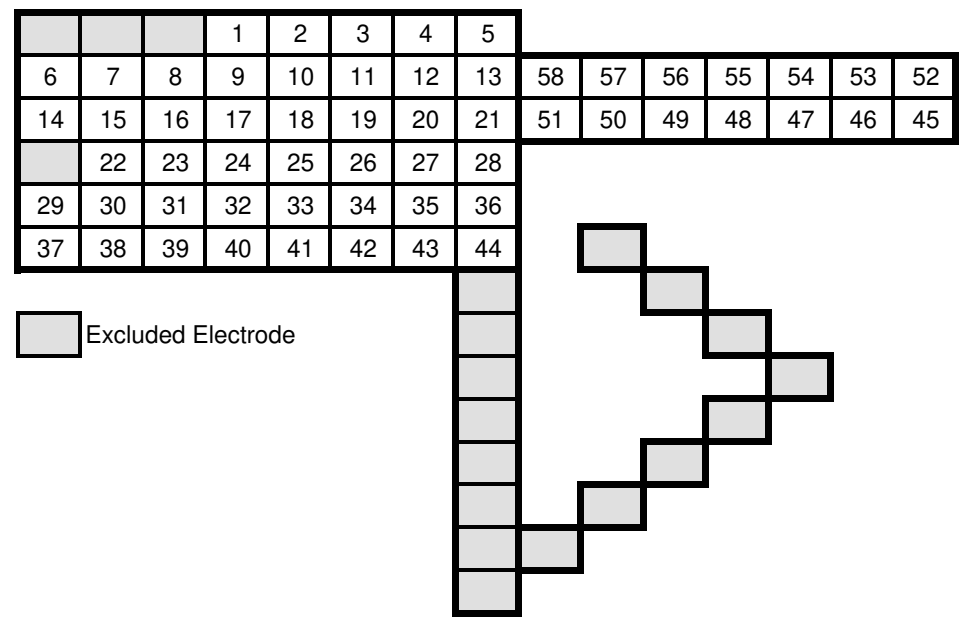

B
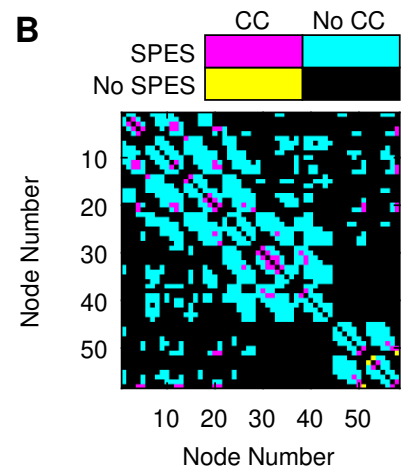

E
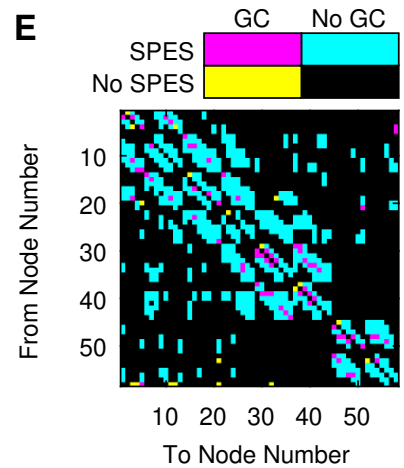

C

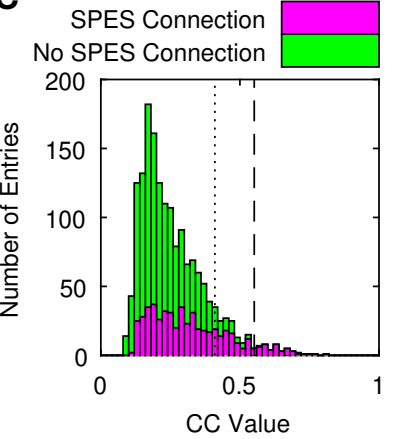

F

F SPES Connection No SPES Connection

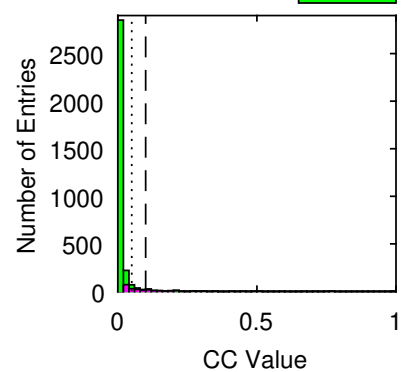

D
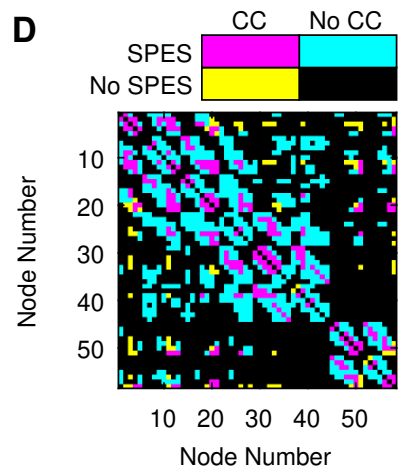

G
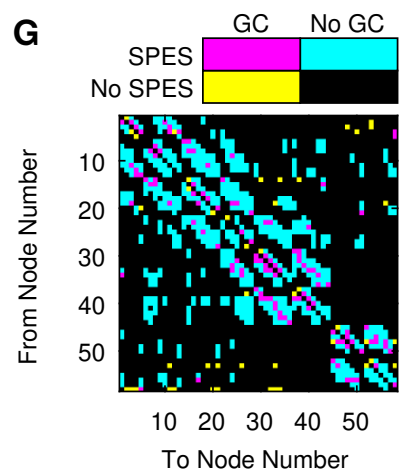

Figure 3.10: Results for patient 4. 
A

\begin{tabular}{|c|c|c|c|c|c|c|c|c|}
\hline & 14 & 15 & 16 & 17 & 18 & 19 & 20 & 21 \\
\hline & 22 & 23 & 24 & 25 & 26 & 27 & 28 & 29 \\
\hline & 30 & 31 & 32 & 33 & 34 & 35 & 36 & 37 \\
\hline & 38 & 39 & 40 & 41 & 42 & 43 & 44 & 45 \\
\hline 1 & 2 & 3 & 4 & 5 & 6 & 7 & & \\
\hline 8 & 9 & 10 & 11 & 12 & 13 & & & \\
\hline
\end{tabular}

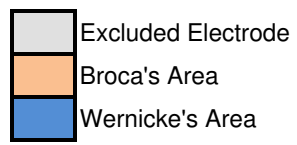

B
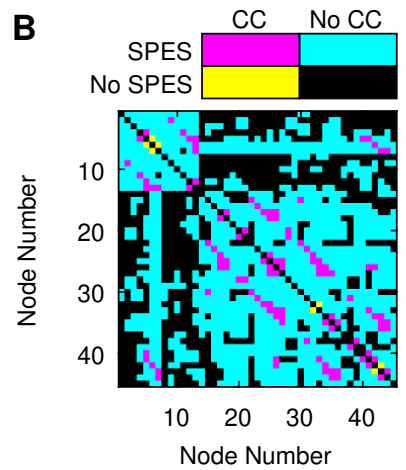

E
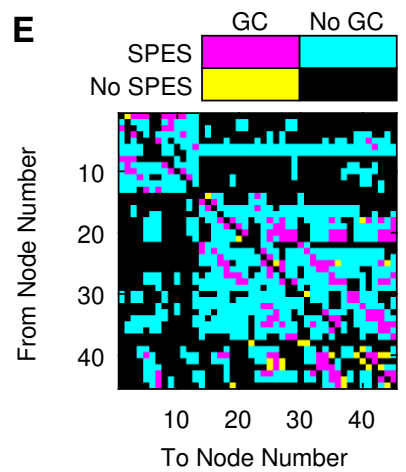

C

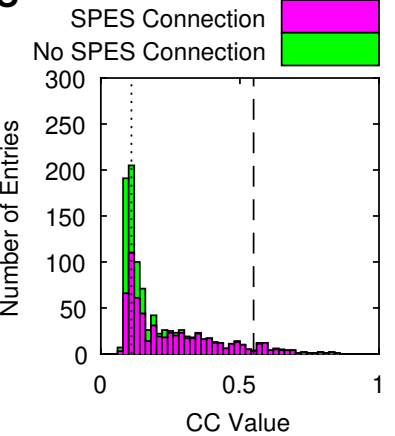

$\mathbf{F}$

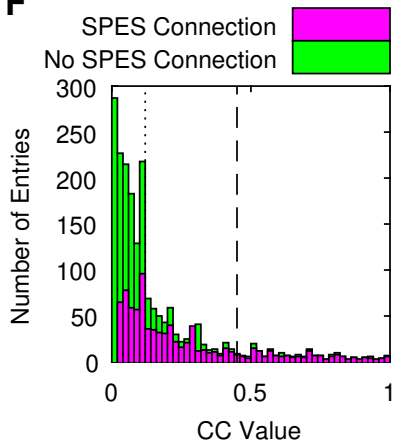

D
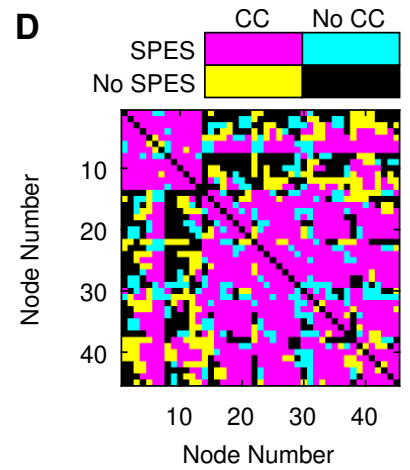

G
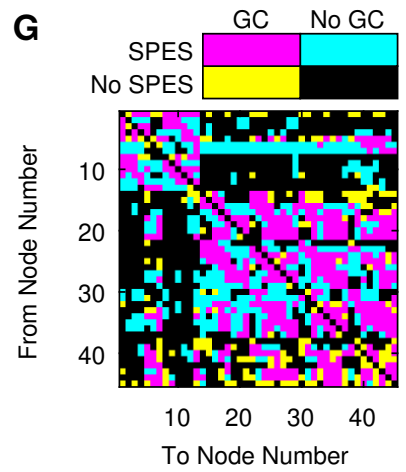

Figure 3.11: Results for patient 5. 
A

\begin{tabular}{|l|l|}
\hline 66 & 58 \\
\hline 67 & 59 \\
\hline 68 & 60 \\
\hline 69 & 61 \\
\hline 70 & 62 \\
\hline
\end{tabular}$\quad \begin{array}{ll}\text { Excluded Electrode } \\
\text { Broca's Area } \\
\text { Wernicke's Area }\end{array}$

\begin{tabular}{|l|l|}
\hline 70 & 62 \\
\hline 71 & 63 \\
\hline 72 & 64 \\
\hline 73 & 65 \\
\hline
\end{tabular}

Wernicke's Area
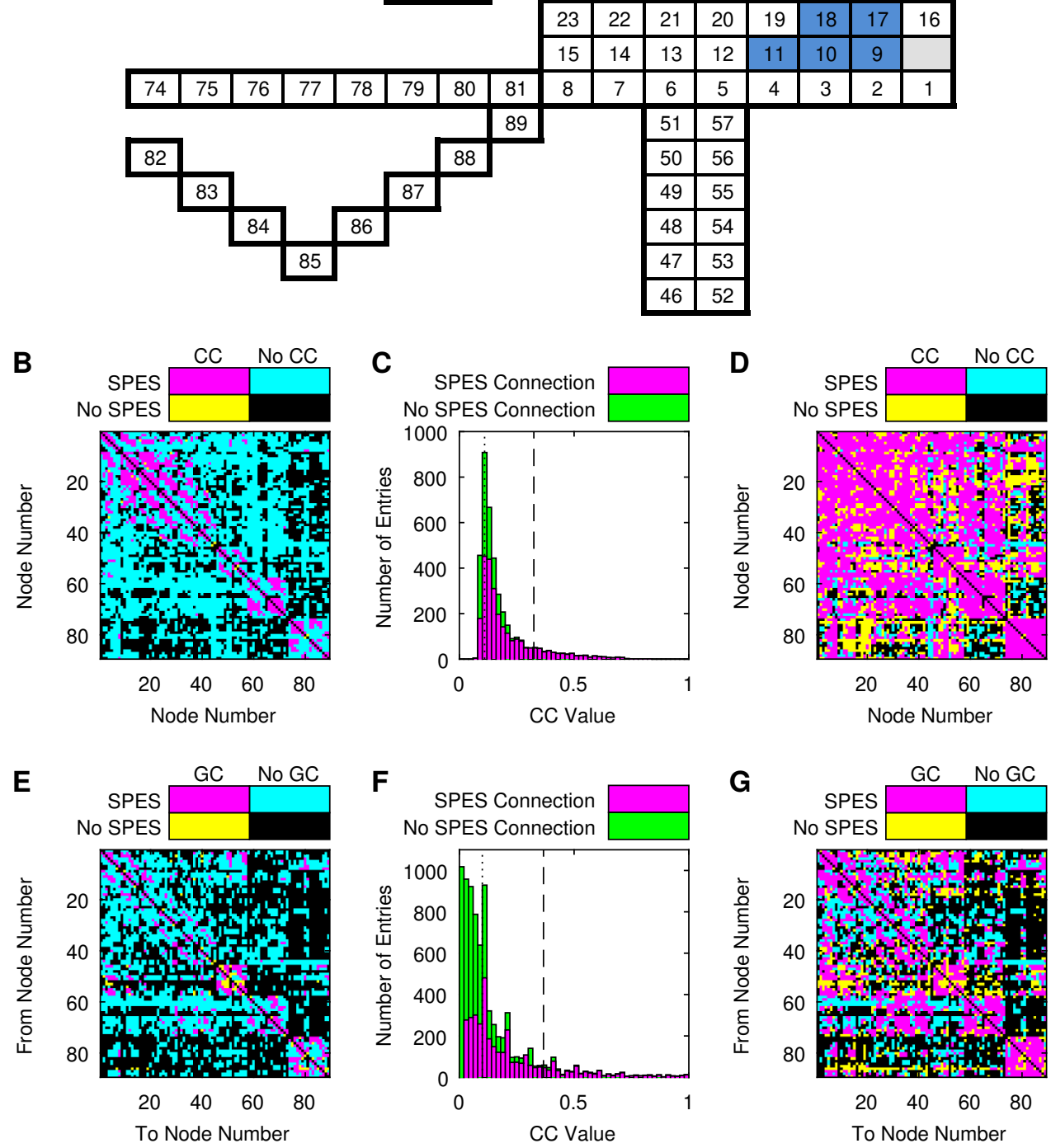

Figure 3.12: Results for patient 6. 


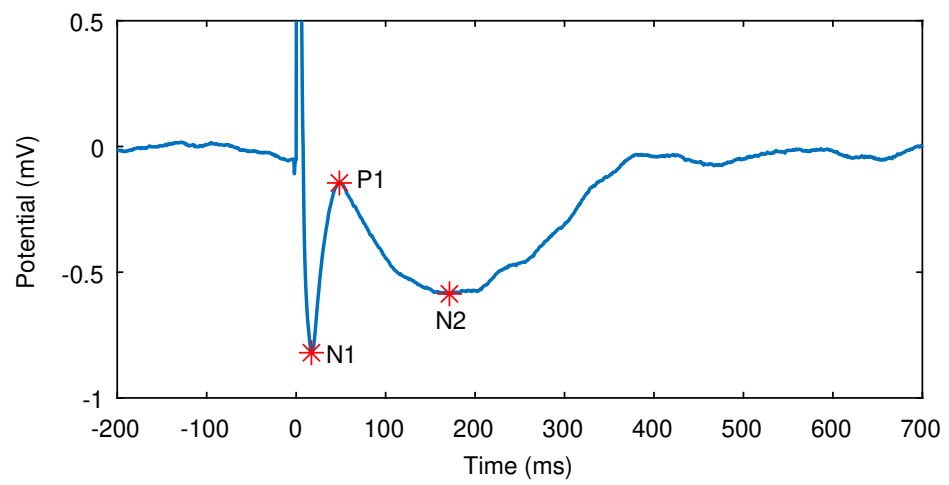

Figure 3.13: Example of a characteristic ER. The three peaks of the ER are called N1, P1 and N2.

peaks, i.e. the N1, P1 and N2 (see Figure 3.13), in the ECoG data averaged over all ten stimulation trials as follows. First all positive and negative peaks in the time range 9-300 ms after the stimulation are detected using the Matlab function peakfinder. This function has a parameter $s$, specifying how much an extreme value should deviate from the neighbouring time points to qualify as an extremum. We put $s=50 \mu \mathrm{V}$ for the positive peaks and, in order to detect the less sharp N2 peaks, $s=20 \mu \mathrm{V}$ for the negative ones. In case there are no positive or less than 2 negative peaks the signal is discarded.

Now, let $\widetilde{P}_{1}<\widetilde{P}_{2}<\ldots$ be the time of the positive peaks and similarly $\widetilde{N}_{1}<\widetilde{N}_{2}<\ldots$ the time of the negative peaks. If $\widetilde{N}_{1}<\widetilde{P}_{1}<\widetilde{N}_{2}$ the detected peaks are in the expected order and we take these peaks as our N1, P1 and N2. Else either $\widetilde{P}_{1}<\widetilde{N}_{1}$ or $\widetilde{N}_{2}<\widetilde{P}_{1}$ holds. In these cases, a spurious peak is detected, e.g. due to noise or spontaneous activity. We try to correct these cases in the following way. If $\widetilde{P}_{1}<\widetilde{N}_{1}$, we check the second positive peak, $\widetilde{P}_{2}$. If this peak lies between the two negative peaks, we accept $\widetilde{N}_{1}, \widetilde{P}_{2}$ and $\widetilde{N}_{2}$ as our N1, P1 and N2. Else we discard the signal. In case $\widetilde{N}_{2}<\widetilde{P}_{1}$, we check if $\widetilde{N}_{3}$ appears after $\widetilde{P}_{1}$. If true, then $\widetilde{N}_{2}, \widetilde{P}_{1}$ and $\widetilde{N}_{3}$ are taken as N1, P1 and N2, else the signal is rejected.

\section{S3.2 Spread of N1 Times}

Figure 3.14 shows the N1 times for a selected stimulation. The N1 times differ much from each other and range between 13 and $34 \mathrm{~ms}$ with a standard deviation of $5.45 \mathrm{~ms}$. Even direct neighbours of the stimulation pair show distributed N1 times. The node left of the stimulation pair (orange border) has a relatively late N1. On the other hand, the node below the right stimulation electrode (green border) has a rather early N1 and a less pronounced N2. From the difference in timing of the N1 peak one can conclude that VC does not play an important role for this stimulation pair.

A different example is shown in Figure 3.15. Here volume conduction seems to play an important role as the spread in N1 times is small. The standard deviation in this case is only $2.45 \mathrm{~ms}$. Most responses are seen approximately $18 \mathrm{~ms}$ after stimulation. Also the shape of the responses is very similar, especially the $\mathrm{N} 1$ and $\mathrm{P} 1$ component.

Next we investigate the effect of VC on a stimulation level. We calculate for each stimulation evoking more than three ERs, the standard deviation of the N1 times. For patient 

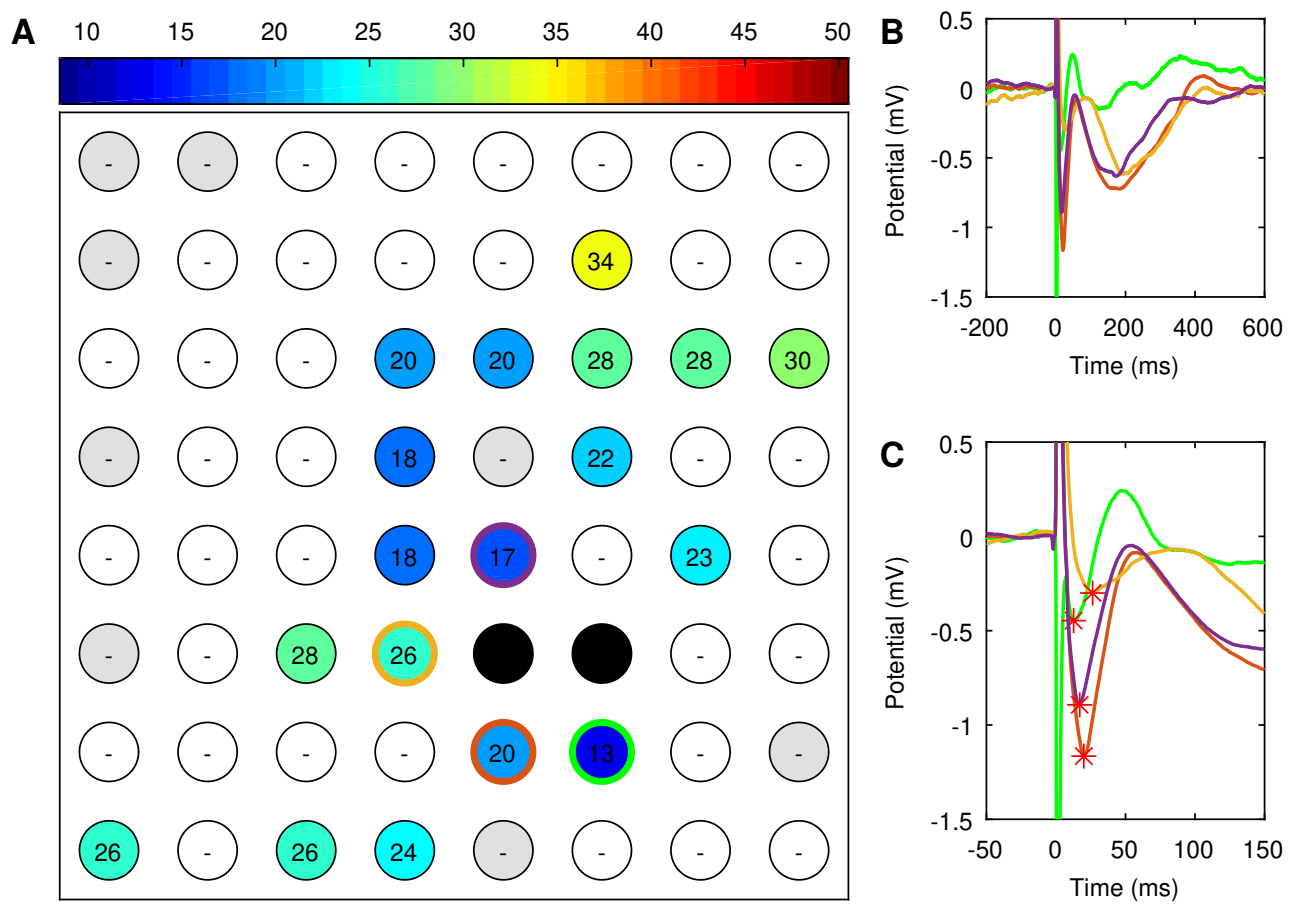

Figure 3.14: N1 times for a selected stimulation. (A) Spatial distribution. Numbers and colours indicate $\mathrm{N} 1$ time of an electrode (in ms). Stimulated and excluded electrodes are coloured black and gray, respectively. (B) Time course of the responses of four selected electrodes. Colours match with the borders of the nodes in $\mathbf{A}$. (C) Close-up of $\mathbf{B}$. 

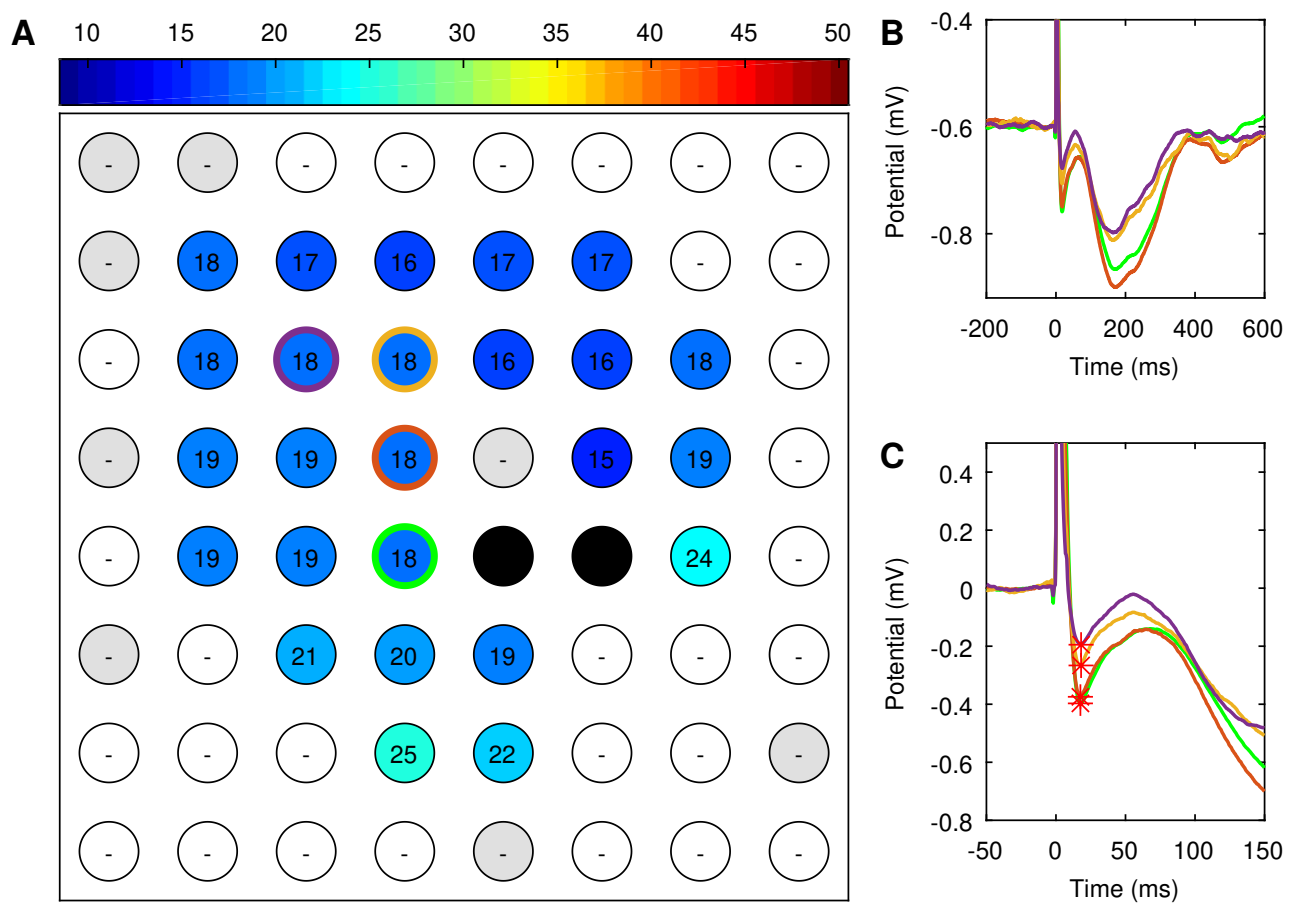

Figure 3.15: N1 times for a selected stimulation. (A) Spatial distribution. Numbers and colours indicate $\mathrm{N} 1$ time of an electrode (in ms). Stimulated and excluded electrodes are coloured black and gray, respectively. (B) Time course of the responses of four selected electrodes. Colours match with the borders of the nodes in $\mathbf{A}$. (C) Close-up of $\mathbf{B}$. 

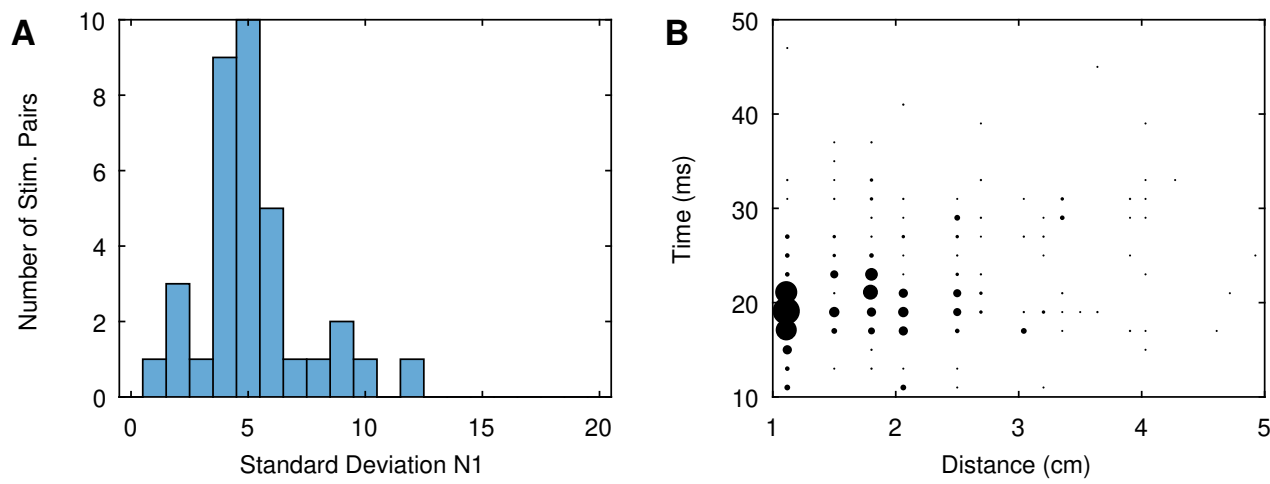

Figure 3.16: Distribution of the N1 peaks in patient 2. (A) Histogram of the stimulation-wise standard deviation of N1 times. (B) Histogram showing the distribution of all N1 over both distance to the stimulation pair and peak time. The size of the dots indicate the number of observations.

2 the distribution of these standard deviations is shown in Figure 3.16A. For most stimulations the standard deviation is around $5 \mathrm{~ms}$, corresponding to the situation in Figure 3.14. In only three cases the standard deviation is low $(<2.5 \mathrm{~ms})$, one of them being the stimulation shown in Figure 3.15. Similar results are found in other patients as shown in Figure 3.17. So most ERs represent local activity, and not distant responses picked up by VC. The latter happens only in a few stimulations.

Finally, we look at the relation between the N1 times and the distance of the response electrode to the stimulation pair. Here, distance is calculated as the Euclidean distance between the electrode and the mid point of the stimulation pair in the schematic grid layout (see Figures 3.1A and 3.8A-3.12A). Results for patient 2 are shown in Figure 3.16B. Observe the rather big spread in N1 times per distance. Further, the N1 time increases slightly with distance. The correlation between N1 time and distance is $0.16(p=0.007)$, for all response electrodes within $3 \mathrm{~cm}$ of the stimulation pair. Results in other patients are comparable, see Figure 3.17. The correlations are $0.36(p<0.001), 0.28(p<0.001), 0.30(p<0.001), 0.15$ $(p=0.011)$ and $0.33(p<0.001)$ for patient $1,3,4,5$ and 6 respectively.

If the observed ERs in SPES were mainly due to VC, then one would expect that distance would not play a role at all for the N1 times, as VC act instantaneously, and hence the correlation would be (almost) zero. On the other hand, if the SPES evoked activity would propagate in all directions homogeneously, then the correlation would have been much closer to one. So the distance to the stimulation pair plays a role in the observed N1 time, but is not the main determining factor.

\section{S3.3 Conclusion \& Discussion}

We conclude that VC plays only a minor role in our SPES data. In only a few stimulations we noticed a (possible) VC effect as N1 peaks appeared at almost the same moment in a large number of electrodes. For most stimulations however, the N1 times were distributed, which implies that VC plays a minimal role in our recordings. The positive correlation between N1 times and the distance to the stimulation electrodes strengthens this conclusion. 
Our results contradict with an earlier study, where VC in SPES was investigated using the root mean square (RMS) of the response [117]. They found that the RMS was proportional to the squared inverse of the distance to the stimulation pair and attributed this finding to VC. An alternative explanation for the observed decay in amplitude could be a distance-dependent decay of connectivity strength. We think therefore that investigating the timing of the N1 responses, like we did, is a more direct approach to investigate the VC effect.

Another explanation for the difference between our results and those of [117] can be the settings of the stimulation. We used monophasic stimulation of $8 \mathrm{~mA}$ and $0.1 \mathrm{~ms}$. In [117] biphasic stimulation with a time of $0.15 \mathrm{~ms}$ per phase was used, which is much longer. Also, the biphasic stimulation might trigger different cortical activity.

We conclude that the effects of VC in our data are limited. VC therefore won't influence (nearby) connectivity in our SPES network much and it is not necessary to account for the effects of VC. 

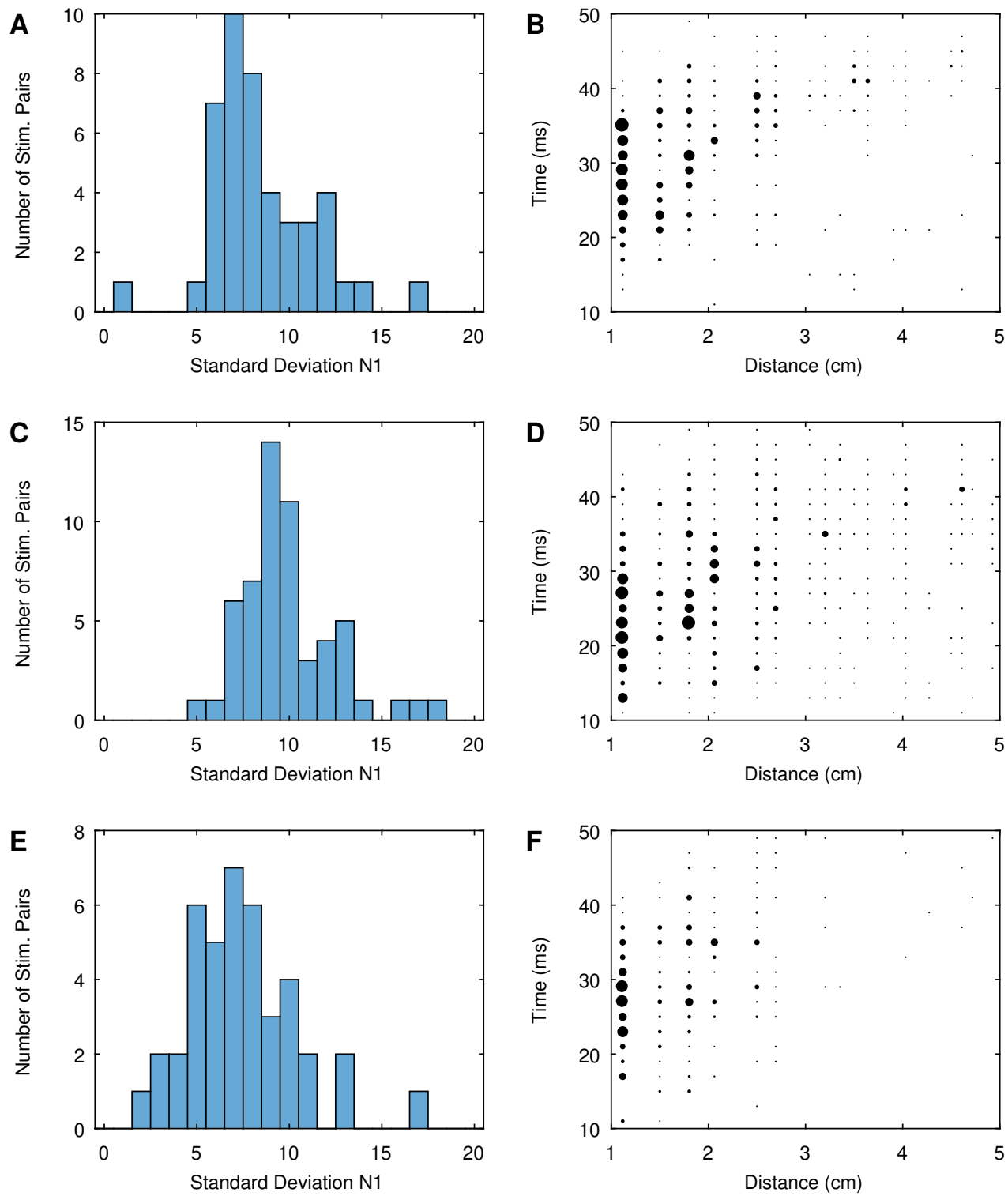

Figure 3.17: (continues on next page) 

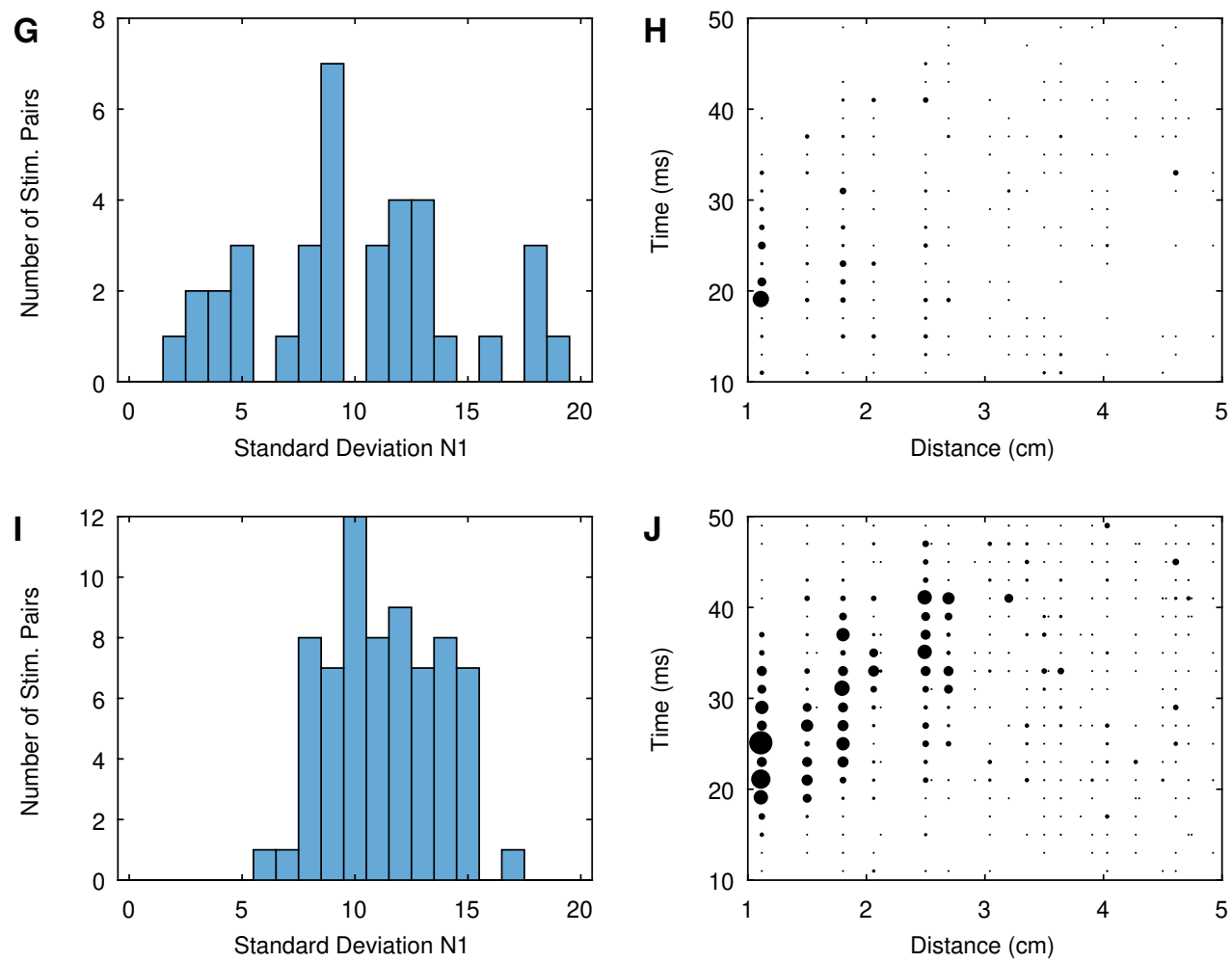

Figure 3.17: Distribution of the N1 peaks for patients 1, 3-6. (A, C, E, G, I) Histogram of the stimulation-wise standard deviation of $\mathrm{N} 1$ times. (B, D, F, H, J) Histogram showing the distribution of all N1 over both distance to the stimulation pair and peak time. The size of the dots indicate the number of observations. 


\section{Chapter 4}

\section{Pathological Responses to Single Pulse Electrical Stimuli in Epilepsy: The Role of Feedforward Inhibition}

Jurgen Hebbink, Geertjan Huiskamp, Stephan van Gils, Frans Leijten, Hil Meijer 


\section{Abstract}

Delineation of epileptogenic cortex in focal epilepsy patients may profit from single pulse electrical stimulation during intracranial EEG recordings. Single pulse electrical stimulation evokes early and delayed responses. Early responses represent connectivity. Delayed responses are a biomarker for epileptogenic cortex, but up till now, the precise mechanism generating delayed responses remains elusive.

We used a data-driven modelling approach to study early and delayed responses. We hypothesized that delayed responses represent indirect responses triggered by early response activity and investigated this for 11 patients. Using two coupled neural masses we modelled early and delayed responses by combining simulations and bifurcation analysis. An important feature of the model is the inclusion of feedforward inhibitory connections.

The waveform of early responses can be explained by feedforward inhibition. Delayed responses can be viewed as second-order responses in the early response network which appear when input to a neural mass falls below a threshold forcing it temporarily to a spiking state. The combination of the threshold with noisy background input explains the typical stochastic appearance of delayed responses. The intrinsic excitability of a neural mass and the strength of its input influence the probability at which delayed responses to occur.

Our work gives a theoretical basis for the use of delayed responses as a biomarker for the epileptogenic zone, confirming earlier clinical observations. The combination of early responses revealing effective connectivity, and delayed responses showing intrinsic excitability, makes single pulse electrical stimulation an interesting tool to obtain data for computational models of epilepsy surgery. 


\subsection{Introduction}

Epilepsy surgery may provide a cure for patients with focal epilepsy, especially if treatment with anti-epileptic drugs fails $[18,122]$. Epilepsy surgery aims at removing the epileptogenic zone (EZ), i.e. the smallest area of cortex the removal of which yields seizure freedom $[20,21]$. Although several non-invasive methods exist to approximate the EZ, the current gold standard remains the seizure onset zone (SOZ) determined via seizures captured during intracranial EEG recordings. The dependence on the occurrence of spontaneous events has the disadvantage that long recording time is therefore needed, typically ranging from a few days up to weeks, which increases the burden of the patient, the risk of complications and costs.

To probe epileptogenicity during electrocorticography $(\mathrm{ECoG})$ registration, single pulse electrical stimulation (SPES) is an alternative for observing spontaneous interictal or ictal changes, with the advantage that it is controlled [28, 40]. During SPES, brief electric pulses are applied directly to the cortex using the electrode grids implanted for clinical ECoG recordings. SPES typically evokes two types of responses: early responses (ERs) and delayed responses (DRs).

ERs appear directly and consistently after the stimulation, with a similar timing and shape across stimulation trials. They are well-understood as they represent brain connectivity [3234]. ERs have, therefore, been used to investigate connectivity in several functional regions like the language area and motor cortex (see [34] for an overview). From ERs, directional networks can be constructed [Chap. 3]. These networks (partly) explain ipsilateral seizure propagation [35, 36], in contrast to contralateral seizure spread [123]. Moreover, network measures calculated from these ER networks exhibit differences between nodes in and outside the resected area and SOZ [37, 38].

DRs appear later than ERs, between $100 \mathrm{~ms}$ and $1 \mathrm{~s}$ after stimulation [28], have a stochastic occurrence, i.e. they only appear on a subset of the stimulation trials at the same electrode pair, and come with variable timing and shape. Where ERs are physiological responses, linked to the EZ only through network structure, DRs are a direct biomarker for epileptogenic cortex $[39,40]$. So far, research on DRs predominantly assessed their clinical value. DRs were observed in different brain regions $[28,29]$ in both adults and children [30]. Further, investigation of the frequency content of DRs revealed that especially high-frequency activity (HFA) in the fast-ripple band $(250-500 \mathrm{~Hz})$ is specific for epileptogenic cortex [40]. Although useful for clinical practice, these results do not give a mechanistic explanation for DRs. Such understanding would provide a better basis for the clinical use of DRs.

Computational models offer a tool to investigate the responses observed during SPES. Neural mass models (NMMs) can simulate EEG-like activity of a small patch of neural tissue ranging from a few millimetres to a couple of centimetres. The development of NMMs dates back to the early seventies to explain generation of the $\alpha$-rhythm in the thalamus [50]. Later NMMs were extended to models for cortical activity [52, 53, 55, 56]. NMMs are able to generate a variety of both healthy and epileptic EEG rhythms [124, 125] and also to describe event-related responses $[51,52,56,58,59]$. Multiple neural masses can be coupled to create an interacting network, allowing the study of network mechanisms. Usually, neural masses have been coupled only through purely excitatory connections, although it is known that multiple connection types exist [126]. Especially feedforward inhibition plays an important role in seizure propagation [127] and responses to transcranial magnetic stimulation [59]. 
In this work we try to explain the two most characteristic properties of DRs, namely their relatively late appearance compared to ERs and their stochastic nature. The first property suggests that DRs might represent indirect, rather than direct, responses to stimulation. These responses may be triggered by ERs, mediated through the ER network. The latter property might be a consequence of noise, which pushes the system only occasionally beyond a threshold for generating DRs. We investigate these hypotheses using both analysis of SPES data and by modelling DRs using a NMM equipped with feedforward connections to inhibitory cells.

First we will summarize properties regarding appearance, timing and amplitude of ERs and DRs. Next, we will investigate if DRs can be seen as indirect responses within the ER network by performing data analysis on SPES data recorded in epilepsy patients. We then present a computational model of DRs which we calibrate by using properties of ERs. Finally, we use bifurcation analysis to reveal a mechanism that explains DRs as second order responses in the ER network including the up to now elusive stochastic appearance of DRs.

\subsection{Materials and Methods}

\subsubsection{SPES acquisition}

At the UMC Utrecht, SPES is performed as part of clinical routine in the pre-surgical evaluation of epilepsy patients during long-term ECoG monitoring. For the ECoG monitoring intracranial electrode grids, usually consisting of $2-8 \times 8$ electrodes and strips $(1 \times 8$ electrodes), are placed directly on the cortex. Electrodes have a circular shape with a contact area of $4.2 \mathrm{~mm}^{2}$ and an inter-electrode distance of $1 \mathrm{~cm}$.

The SPES protocol has been described in detail [38, 40]. In short, pairs of adjacent electrodes along the length of the grid receive ten monophasic electrical pulses with a duration of $1 \mathrm{~ms}$, at an interval of $5 \mathrm{~s}$ and a typical intensity of $8 \mathrm{~mA}$. During SPES, ECoG data is recorded with respect to an extracranial reference located on the contralateral mastoid at a sampling rate of $2048 \mathrm{~Hz}$ using a SD LTM express (MicroMed, Veneto, Italy). Stimulations are applied via the LTM stimulator and cause a 9 ms lasting artefact in all signals. Further, the stimulated channels become saturated for about $5 \mathrm{~s}$ upon stimulation, so responses can only be observed in the remaining electrodes.

\subsubsection{ERs}

SPES ERs are defined as responses starting within $100 \mathrm{~ms}$ after stimulation. ERs are normal, physiological brain responses describing connectivity [28, 32-34]. The most common type of ER (Figure 4.1A) consists of three peaks, which are in order of appearance: N1, P1 and N2 [31], while in other ERs (Figure 4.1B) the last component is absent. The N1 is a sharp negative peak, occurring roughly between 10 and $50 \mathrm{~ms}$ after stimulation [96], with the majority around $15 \mathrm{~ms}$, see Figures 4.1C, D. The P1 is the positive deflection following the N1. Its maximum lies around baseline level and typically occurs $\sim 35 \mathrm{~ms}$ after the N1. The timing of both the $\mathrm{N} 1$ and P1 is similar for ERs with and without N2 component. The broad negative slow wave following the P1 is the N2. Its waveform is more variable compared to N1 and P1 peaks as it is shaped by spontaneous activity too. The biggest amplitude is attained some 80 to $160 \mathrm{~ms}$ after the N1 with the median around $110 \mathrm{~ms}$, see Figures 4.1C. The ratio between N1 and N2 

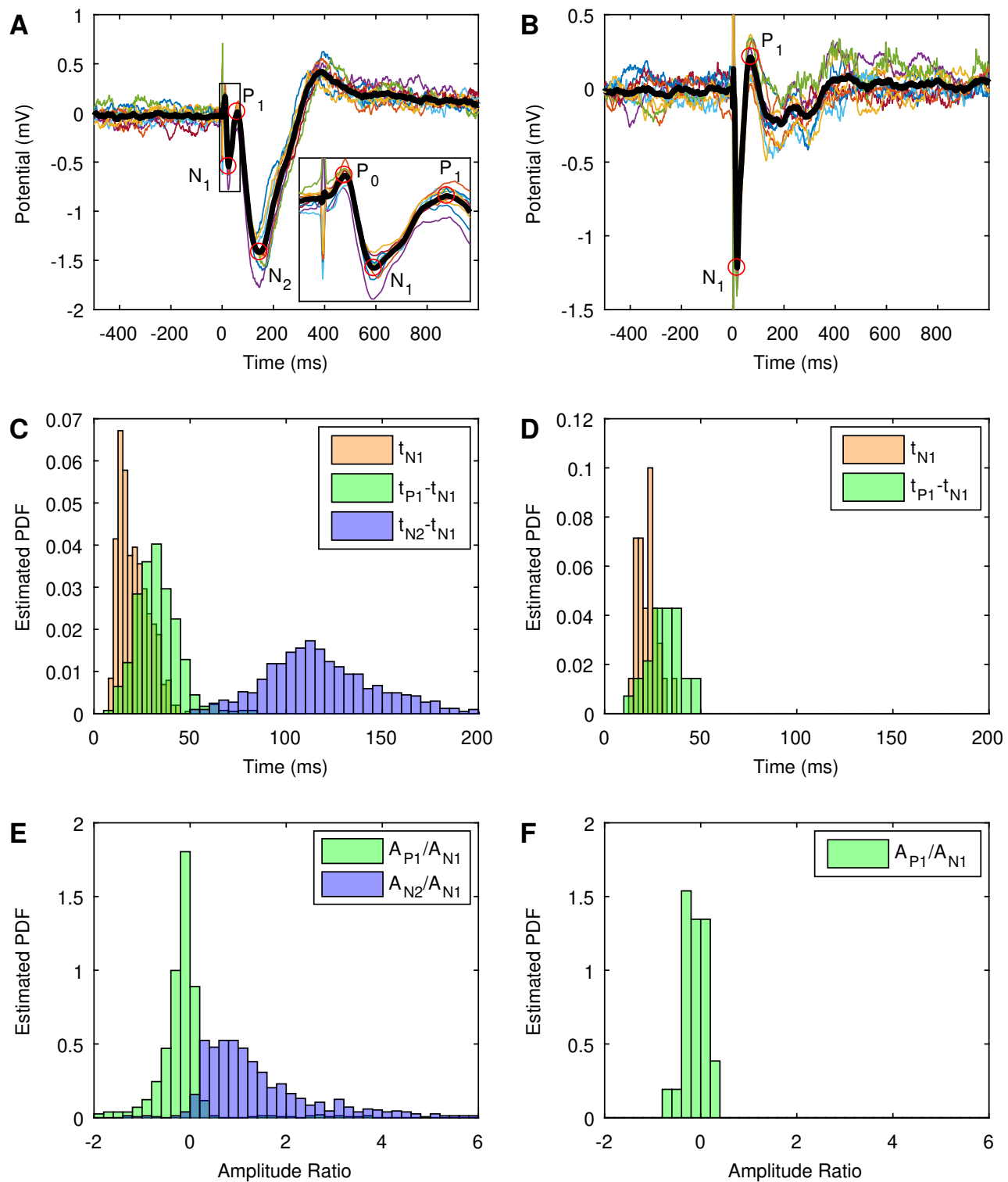

Figure 4.1: Shape and properties of early responses (ERs) in vivo. (A, B) Examples of an ER with and without N2, respectively. Thin coloured lines are multiple trials for the same stimulation. The thick black line indicates the average response. The inset in $\mathbf{A}$ shows a magnification of the response, here the P0 peak is visible. (C, D) Distribution of the N1, P1 and N2 times for ERs with and without N2, respectively. Time of $\mathrm{N} 1$ peaks is the time after stimulation, $\mathrm{P} 1$ and $\mathrm{N} 2$ times are relative to N1. (E, F) Amplitude of the P1 and N2 peaks relative to that of the N1. Amplitudes are taken as the deviation from the mean activity $50 \mathrm{~ms}$ before stimulation, hence $\mathrm{N} 1$ and $\mathrm{N} 2$ peaks usually have a negative value. 
amplitude varies (Figures 4.1E). For some stimulation pairs and response electrodes the N2 is smaller than the N1, while for others it is much larger.

In some cases, we observed an additional positive peak preceding the $\mathrm{N} 1$ peak, see the inset of Figure 4.1A for an example, which we call P0. Such a peak has also been reported in $[34,128]$. In this example the P0 peak is found $11.2 \mathrm{~ms}$ after stimulation with an amplitude of 0.4 times that of N1. The P0 peak is difficult to find in the data, due to its relatively low amplitude and because it mixes with the stimulation artefact (up to $9 \mathrm{~ms}$ ) in most cases. So, only if the N1 peak is relatively late, there is a time window in which such a P0 may be noticed.

To systematically detect ERs in our SPES data we use an automatic detector. This detector is described in detail in 3.S1 and has been validated using visually annotated ERs. In short, the responses of an electrode over all ten trials of a stimulation pair are averaged. If the extremum of this signal between 9 to $100 \mathrm{~ms}$ after stimulation sufficiently exceeds the standard deviation of the baseline, i.e. the $2 \mathrm{~s}$ prior to stimulation, the response is classified as ER.

\subsubsection{DRs}

DRs are responses to SPES appearing at least $100 \mathrm{~ms}$ after stimulation (see Figure 4.2). DRs occur only in a subset of the trials, e.g. in Figure 4.2A DRs occur in trials 1, 2, 5, 6 and 9, around 200-400 ms after stimulation. The exact timing of DRs differs per trial and is even more variable across stimulation pairs and electrodes. For example, DRs in Figure 4.2B start around $400 \mathrm{~ms}$ after stimulation, while the others in Figure 4.2 start around $200 \mathrm{~ms}$.

The waveform of DRs varies substantially across electrodes. The DRs shown in Figures 4.2A-C have been recorded from the same electrode. These DRs start with rapid oscillations and are, except for some trials in Figure 4.2C, followed by a slow wave. DRs recorded from other electrodes look more like spike-wave discharges as those in trials $1,3,5$ and 6 of Figure 4.2D. Interestingly, trials 8, 9 and 10 of this stimulation exhibit DRs similar to those in Figure 4.2A, so DR waveforms may vary even within the same stimulation for the same electrode. Note also that an electrode may show both an ER and DR simultaneously as can be seen in Figure 4.2F.

Both the variable timing and varying waveform render averaging responses in the time domain problematic. Typical analysis of DRs employs time-frequency analysis using a Morlet wavelet transformation and averages the resulting time-frequency plots [40]. Time-frequency plots allow classification of DRs with components in the spike $(10-80 \mathrm{~Hz})$, ripple $(80-250 \mathrm{~Hz})$ and fast-ripple $(250-500 \mathrm{~Hz}$ ) bands (see Figure 4.2E). We determine the presence of DRs per frequency band by visual analysis of the time-frequency plots, see [40] for details. In short, for each channel a time-frequency image is produced per stimulation pair which is independently scored by two observers in the three frequency bands. Only responses on which the two observers agreed are considered. Inter-observer agreement is assessed via Cohen's $\kappa$ and is considered to be reasonable if $\kappa>0.4$. Here we study DRs that cover at least the spike and ripple band. The presence of a fast-ripple is not required as these occur rarely [40].

Next, the onset time of DRs is determined by visual analysis of the single trial responses. For each trial a time-frequency image is produced using the same settings as used for the averaged time frequency images. The onset is then marked as the first time point where both increased activity in the time frequency image and a clear onset of the DR in the time signal is visible. We compare the onset time between two groups of DRs, i.e. those which 
A
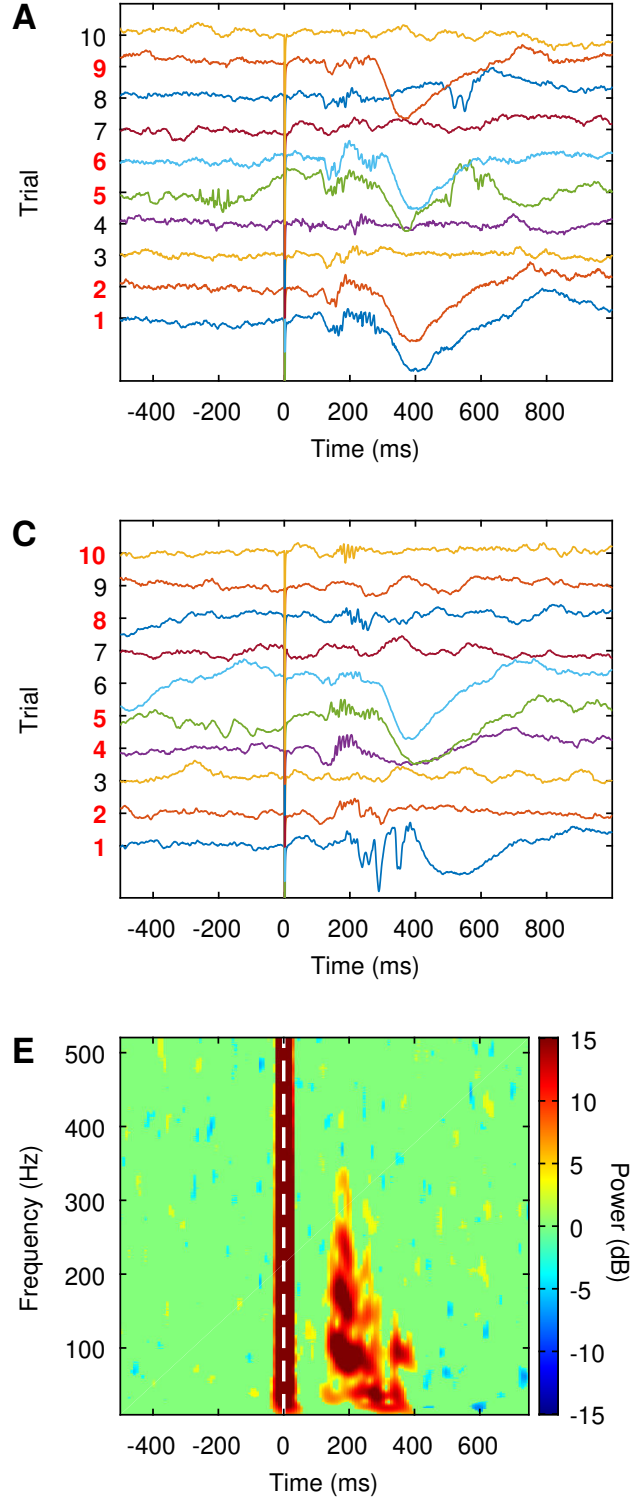
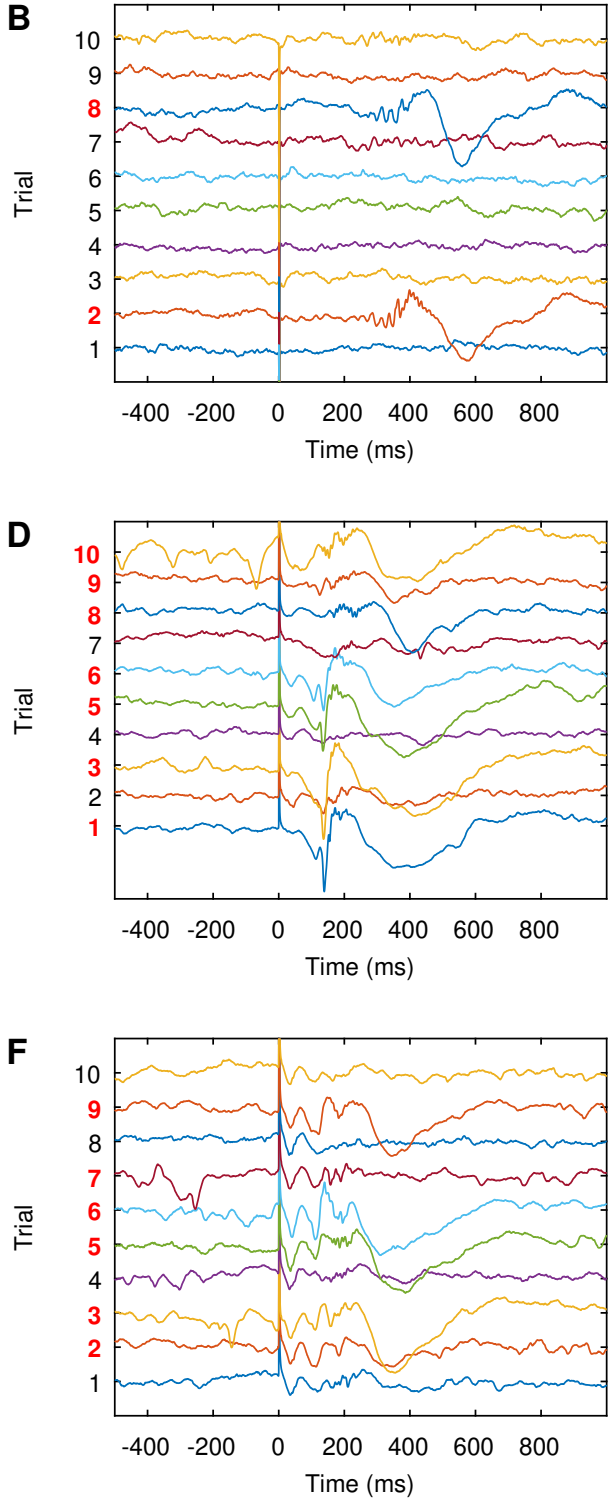

Figure 4.2: Examples of delayed responses (DRs) in vivo. (A-F) Response of a single electrode to ten subsequent trials of the same stimulation pair. Red-coloured trial numbers indicate DRs. Responses at $\mathbf{A}-\mathbf{C}$ were recorded at the same electrode, the same hold for the responses in $\mathbf{D}, \mathbf{F}$. (E) Averaged time-frequency response of the trials in $\mathbf{C}$.

are preceded by an ER, and those that are not. Using the Wilcoxon rank-sum test (function ranksum in Matlab) we test whether the median values of these two groups are equal against the alternative hypothesis that the median onset time of DRs preceded by an ER is later. We 
reject the null hypothesis for $p<0.05$.

\subsubsection{Path Length of DRs in ER Networks}

We use the detected ERs to construct a network representing effective connectivity [Chap. 3, $33,38,96]$. Each electrode represents a node in this network. An edge from node A to B is present if stimulation involving electrode A evokes an ER at electrode B. The result is an unweighted directional network.

Next, we study the distance from stimulation pairs to the electrodes on which they evoke DRs in the ER network. We define distance as the length of the shortest path between a stimulation pair and an electrode. Starting from a stimulation pair all electrodes on which an ER is evoked are at a distance one from the stimulation pair. Next, a node has distance two from the stimulation pair if it can be reached via some edge from a node with distance one in the ER network, provided the node itself did not exhibit an ER. Continuing this way, a node is at distance $n$ from the stimulation pair if it is not at distance $n-1$ or closer and it can be reached via an edge from a node at distance $n-1$. Nodes that cannot be reached in this way have an infinite distance to the stimulation pair. This does not imply that the tissue under such an electrode is disconnected from the tissue under the stimulated electrodes as the electrode grid only samples a part of the brain and it might be connected via an uncovered part of the brain.

We investigate the distance from stimulation pair to DR electrode in SPES data of 11 patients recorded during long-term ECoG monitoring at the UMC. For each patient we calculate the percentage of DRs at a distance of at most $1,2,3$ up to $n_{e l}-2$ from the stimulation pair in the ER network, where $n_{e l}$ is the number of electrodes. In all patients SPES was performed for clinical reasons with the protocol described above. Patients had been admitted to the intensive epilepsy monitoring unit of the University Medical Centre of Utrecht, the Netherlands. All patients gave prior informed consent which was recorded in the patient's electronic file and the entire investigation was performed under the approval of the UMC Utrecht's ethical committee under Dutch law. Data were retrospectively collected and handled coded and anonymously according to the guidelines of the institutional ethical committee following the principles of good clinical practice and adhering to the Declaration of Helsinki.

\subsubsection{Mathematical Model}

To model the observed ECoG responses to SPES we use a system of coupled neural masses. Each of these neural masses can be thought to model the tissue underneath an electrode of the ECoG grid. We consider an extended version of the neural mass proposed by [53], see Figure 4.3A. This neural mass models the average membrane potential of four neuronal populations i.e. pyramidal cells (py), local excitatory cells (ex), slow inhibitory cells (is) and fast inhibitory cells (if). As pyramidal cells are the main contributor to EEG signals, their average membrane potential is considered to be proportional to EEG signals $[52,53,59,125]$ and taken as the output of the model.

The average membrane potential of a population determines the activity of that population, i.e. the mean firing rate of the neurons in the population, via a non-linear function $S(u)$ (see Figure 4.3B). Following [56, 58, 129], we shift this function such that $S(0)=0$, to model 

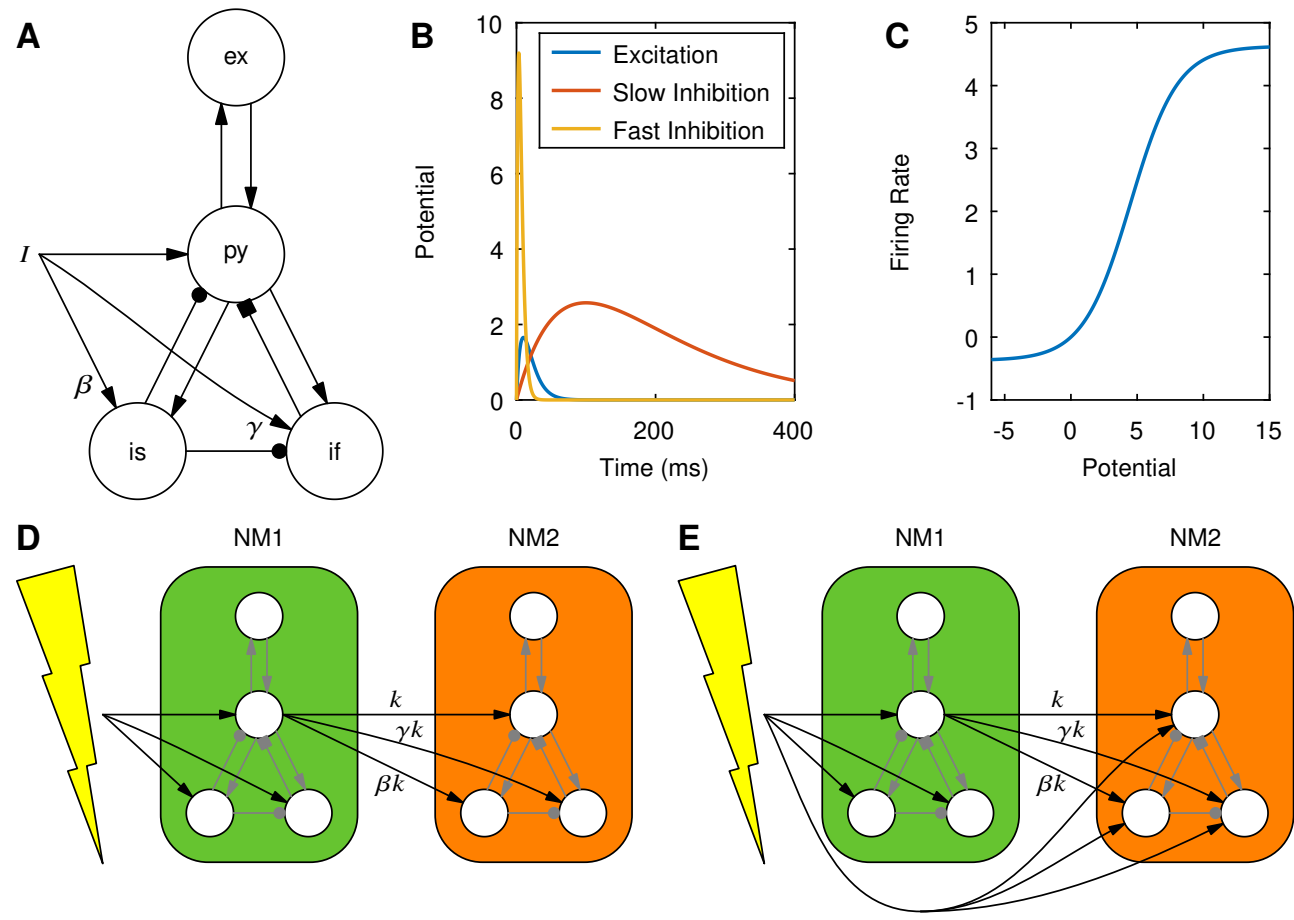

Figure 4.3: (A) Architecture of a single neural mass. The circles represent the four populations, i.e. pyramidal cells (py), local excitatory cells (ex), slow inhibitory cells (is) and fast inhibitory cells (if). Arrows represent excitatory connections, circles and squares are slow and fast inhibitory connections. (B) Graph of the sigmoid function used to convert the average membrane potential of a population into a firing rate. (C) Impulse response of the different synapse types. (D, E) The two configurations of feedforward coupled neural masses considered in this work.

deviations from the normal activity. Accordingly, negative values are interpreted as activity below baseline.

The activity of a population influences the mean membrane potential of other populations via synaptic transmissions. Such synaptic transmission converts the mean firing rate of the sending population into a postsynaptic potential at the receiving population and is modelled by a linear, second order differential equation. The time course of the synaptic transmission is determined by the rising time of the synapse. Following $[52,53]$ we set the rising time for excitatory synapses to $10 \mathrm{~ms}$. For the fast inhibition, modelling fast $\mathrm{GABA}_{\mathrm{A}}$ transmission, we take a rising time of $3.3 \mathrm{~ms}$, which is inside the physiological plausible range reported by [130]. Finally, we assume that slow inhibition models mainly transmission of slow $\mathrm{GABA}_{\mathrm{A}}$ but also partly transmission of $\mathrm{GABA}_{B}$. We therefore set this time scale to $100 \mathrm{~ms}$, which is slightly slower than 30 to $70 \mathrm{~ms}$ for $\mathrm{GABA}_{\mathrm{A}}$ but faster than the 200 to $400 \mathrm{~ms}$ for $\mathrm{GABA}_{\mathrm{B}}$ as suggested by [130]. The impulse responses of the three different synapse types are shown in Figure 4.3C.

In each neural mass the pyramidal cells play a key role, as they are reciprocally connected to all other populations. In addition, slow inhibitory cells project to fast inhibitory cells. 
The relative strength of the connections is set to commonly used values [53] as derived previously from anatomical studies [52]. To arrive at absolute connection strengths, these relative strengths are multiplied by a constant $C$, governing the general internal connectivity. This $C$ can also be seen as a measure for the excitability of a neural mass as increasing $C$ results in more epileptiform dynamics of the neural mass [60,131].

Apart from local interactions neural masses also receive excitatory external input. In the original model this input projects only to the pyramidal cells. Here, also the inhibitory populations receive external input. We model the strength of the feedforward inhibitory input relative to the feedforward excitatory input, using scaling constants $\beta$ and $\gamma$ for slow and fast populations, respectively. External input originates from three sources, i.e. other neural masses, background activity and SPES. Input from other neural masses depends on the activity of their pyramidal cells and connection strength. Background inputs are modelled by independent Gaussian white noise with zero-mean and standard deviation $\sigma$. SPES input to a neural mass is modelled as a short, transient external input (block pulse). This input mimics the activation of the outgoing fibres of a stimulated region which are thought to be activated during SPES rather than the cell bodies [33, 132]. Therefore, SPES input is given simultaneously to all neural masses connected to the stimulated area.

We consider two feedforward coupled neural masses in two different configurations (see Figures 4.3D, E). In both configurations the first neural mass receives SPES input and has a connection with strength $k$ to the second neural mass. Depending on the case, SPES input to the second neural mass might be present or absent. Following our hypothesis, we intend to model an ER on the first and a DR on the second neural mass. Depending on the configuration this DR is preceded by an ER on the second neural mass or not, capturing some of the different cases seen in the data (see Figure 4.2). In both cases, the complete system comprises a set of 20 coupled differential equations (see $4 . \mathrm{S} 1$ ).

Our first modelling step concerns simulating a realistic ER, both with and without a N2 component. For this part we adapt the input strengths to the two inhibitory populations, $\beta$ and $\gamma$. Once these parameters are set, we proceed with modelling DRs by varying the connectivity strength between the neural masses. We perform bifurcation analysis using Matcont [133] to infer a mechanism explaining the stochastic occurrence of DRs. Next, we add background noise to the model, which we neglect in the first part, to show a stochastically occurring DR. Finally, we study how the excitability of the second neural mass and the connection strength influence the rate at which DRs occur.

\subsection{Results}

\subsubsection{Data Analysis}

The relatively late appearance of DRs compared to ERs after stimulation suggests that DRs could be indirect responses due to propagation of activity via the ER network. Example data supporting this hypothesis is shown in Figure 4.4A. SPES delivered at an electrode pair (yellow nodes) evokes ERs at green nodes. These green nodes correspond to all outgoing connections of the stimulation pair in the ER network. Also, the same stimulation evokes DRs, for instance at the orange-coloured electrode. Next, blue nodes indicate that if that electrode is stimulated then the orange node shows an ER, hence they correspond to all nodes that project to the orange node in the ER network. The overlap is indicated by the green-blue 

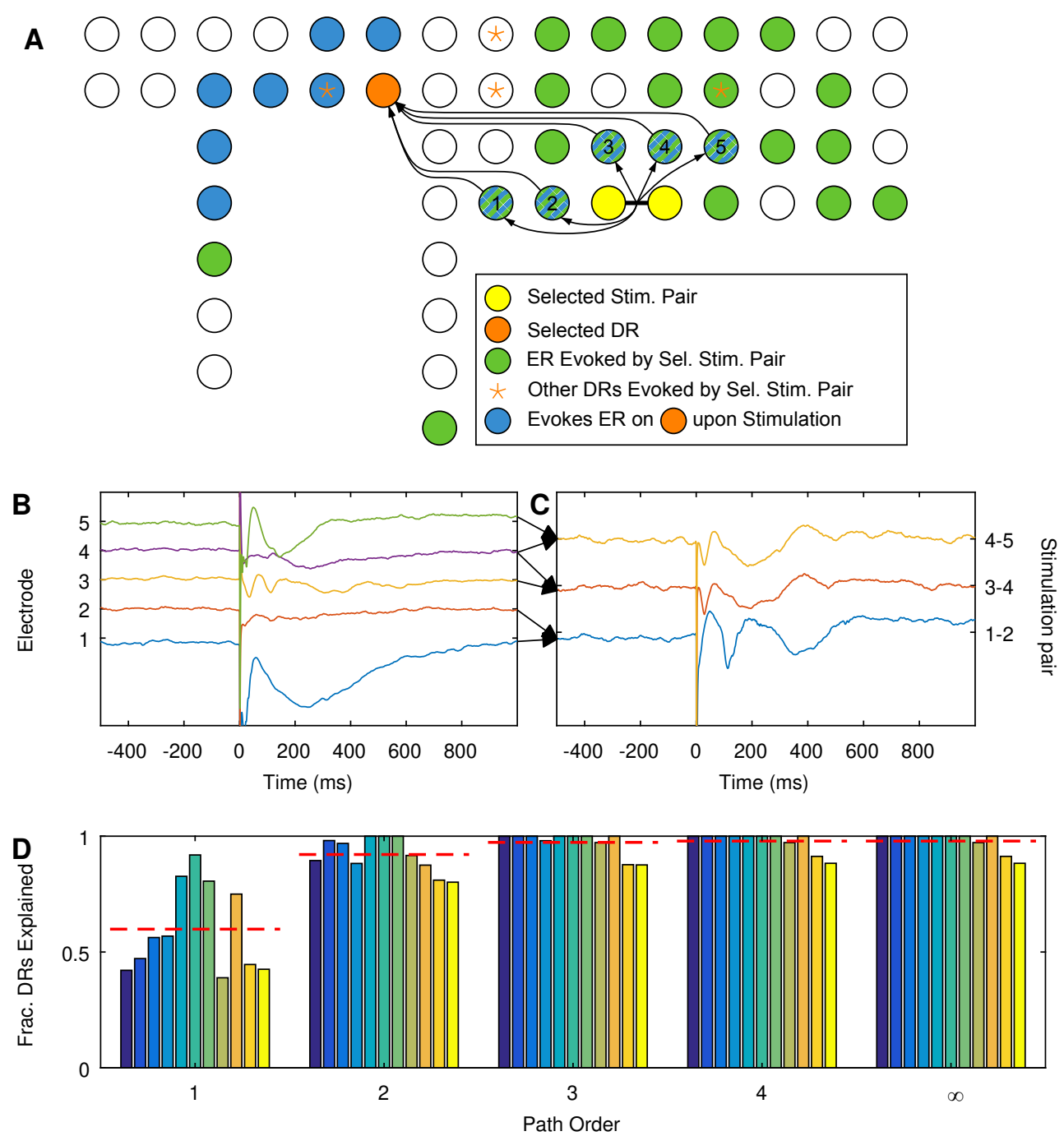

Figure 4.4: Delayed responses (DRs) as indirect response in a patient. (A) Example of a DR as second order response in a schematic overview of the electrode grid. The stimulation pair (yellow) induces a DR on the orange electrode (time trace shown in Figure 4.2C) and early responses (ERs) on all green (solid + striped) electrodes. Blue (solid + striped) electrodes are part of stimulation pairs that induce ERs on the magenta electrode. The arrows indicate length two paths between stimulation pair and DR electrode. (B) Responses of the green-blue striped electrodes on stimulation of the yellow stimulation pair. (C) Responses of the orange electrode on stimulation pairs that consist of the green-blue striped electrodes. (D) Fraction of DRs that can be reached by a path of a certain length in the ER network, where order $\infty$ means that the DR can be reached by a path of arbitrary length. For each path order the 11 bars represent the results of the 11 patients, while the red dashed line indicates the mean over all patients. 
striped pattern. We see that in this network there are multiple, i.e. 5, paths of length two in the ER network from the stimulation electrodes to the electrode showing a DR. These length-two paths are the shortest routes between the stimulation pair and the orange electrode in the ER network, as the orange electrode does not show an ER for this stimulation pair (see also the response of this electrode in Figure 4.2C). Therefore, the orange electrode is at a distance two from the stimulation pair.

Next, we investigate the distance between stimulation pair and DR in 11 patients. The bar chart in Figure 4.4D shows the fraction of DRs that can be reached by a path of a certain length. For most patients, a path of length one, i.e. an ER and DR are seen on the same electrode, explains only half of the DRs. With a path of at most length two, one reaches on average $92 \%$ of the DRs, which is rather constant over all patients. A path length of three or four gives a slight improvement, but higher path lengths do not add anymore. This suggests to model DRs as second order responses in the ER network. The simplest network architecture satisfying this constraint is a feedforward network consisting of two nodes, which we will consider in this work.

\subsubsection{Modelling ERs}

To model ERs we consider a single neural mass receiving a short transient input (see Figure 4.3A). An example of a simulated ER is shown in Figure 4.5A. Here, the thick line indicates the output of the model, i.e. the simulated potential of the pyramidal cells. Note that the simulated ER has a similar shape as those observed in the data. The latency of the P0, $\mathrm{N} 1, \mathrm{P} 1$ and N2 peaks is also realistic as they are found 6, 23, 50 and $118 \mathrm{~ms}$ after stimulation respectively.

The potential of the pyramidal cells is the sum of the contributions from the other populations and the SPES input, which are indicated by the thin coloured lines in Figure 4.5A. The initial increase in the potential of the pyramidal cells is a direct consequence of the stimulation. As the stimulation has a similar effect on both inhibitory populations, these populations are activated and start to influence the pyramidal cells. First, input from the fast inhibitory population comes into play yielding the $\mathrm{N} 1$ peak. The effect of the slow inhibitory population appears much later and results in the $\mathrm{N} 2$ peak. The local excitatory cells only play a minor role in the generation of ERs as they are not directly activated by the SPES stimulus. The amplitude of the ER depends approximately linearly on the stimulation strength as is shown in Figure 4.5B.

The external input to the inhibitory cells is the crucial aspect for the occurrence of the N1 and $\mathrm{N} 2$ peaks. Figures 4.5C, 4.5D show the influence of variations in $\beta$ and $\gamma$ respectively. Variations in $\beta$ only affect the amplitude of the N2 peak, which scales approximately linear with $\beta$. As a consequence, ERs without N2 component can be modelled by setting $\beta=0$. Variations of $\gamma$ affect mostly the N1 peak. For moderate values of $\gamma$ the N1 peak scales approximately linear. For low values of $\gamma$ the N1 peak occurs at the same time, but as the amplitude of the peak is small, the neural mass proceeds differently to the N2. For high values of $\gamma$ the amplitude of the N1 peak grows only slowly, while the latency increases slightly. 

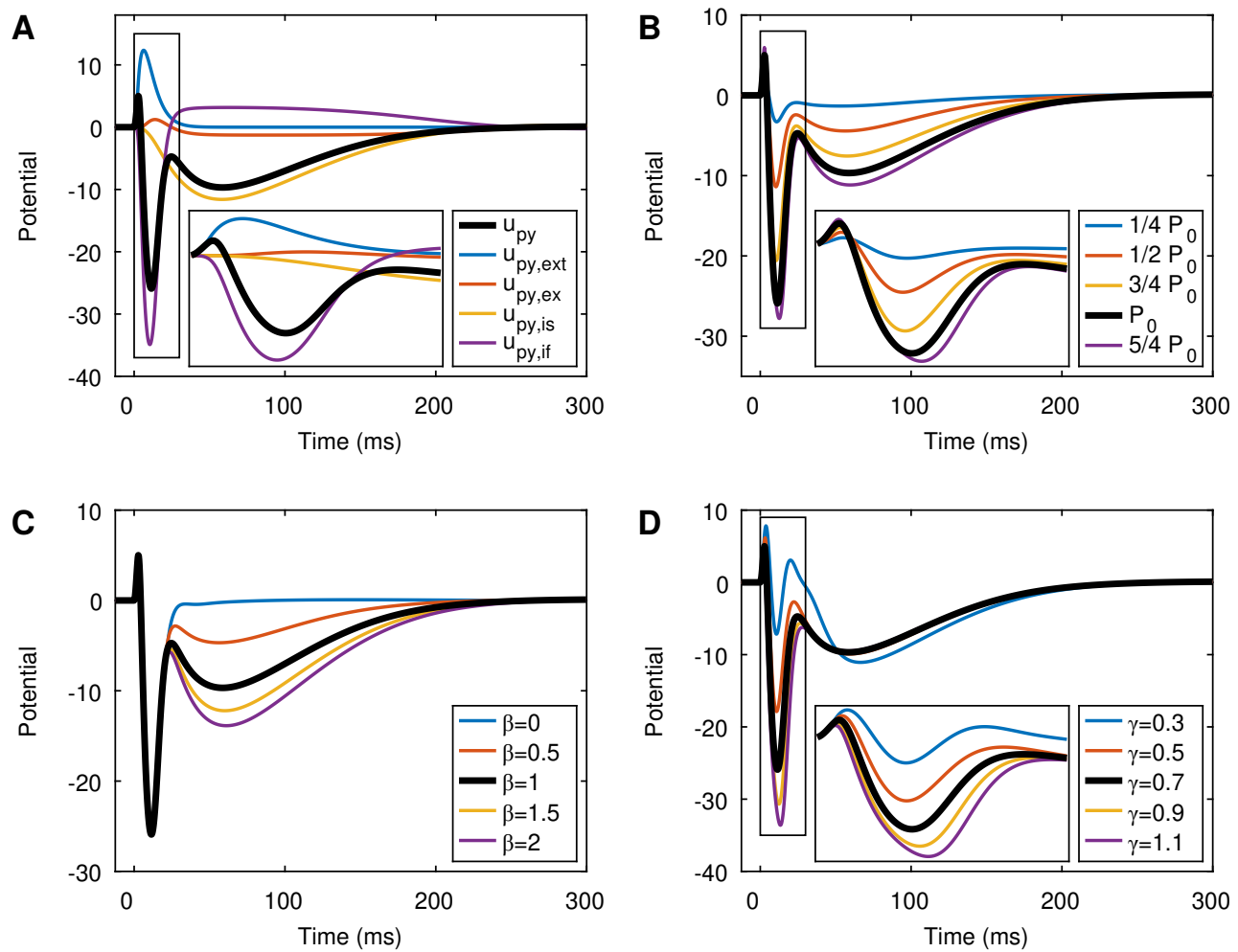

Figure 4.5: Simulated early responses (ERs). (A) Simulated ER using default parameters (thick black line). Thin coloured lines indicate the 4 different sources, i.e. external input (ext) and input from excitatory (ex), slow inhibitory (is) and fast inhibitory (if) populations, adding up to the potential of the pyramidal cells (py). (B) Variation of stimulation strength, where $P_{0}=1500$ is the default strength (indicated with the tick black line). (C) Influence of varying $\beta$, the fraction of external input added to slow inhibitory cells. (D) Influence of varying $\gamma$, the fraction of external input added to the fast inhibitory cells.

\subsubsection{Modelling DRs}

We now consider two feedforward coupled neural masses, as depicted in Figure 4.3D, where the first neural mass projects to the second with connectivity strength $k$. The first neural mass shows ER activity upon stimulation. Figure 4.6A shows the reaction of the second neural mass for various $k$ values. For low values of $k$, the second neural mass shows virtually no response. For $k \gtrsim 20$, a large response, comprising a few oscillations followed by a slow wave, appears. This waveform is qualitatively similar to the DRs in Figures 4.2A, B, although the frequency of the oscillations in the limit cycle are slower, i.e. $\sim 28 \mathrm{~Hz}$ compared to the high-frequency activity ( $>80 \mathrm{~Hz}$ ) at the start of the DRs.

A possible way to model an ER succeeded by a DR is to apply SPES input to both NMMs as in Figure 4.3E. For this, $k$ needs to be large, i.e. $k \gtrsim 75$ (see Figure 4.6B). The shape of this DR is similar to the DRs that are not preceded by an ER. For $k$ values below the threshold 

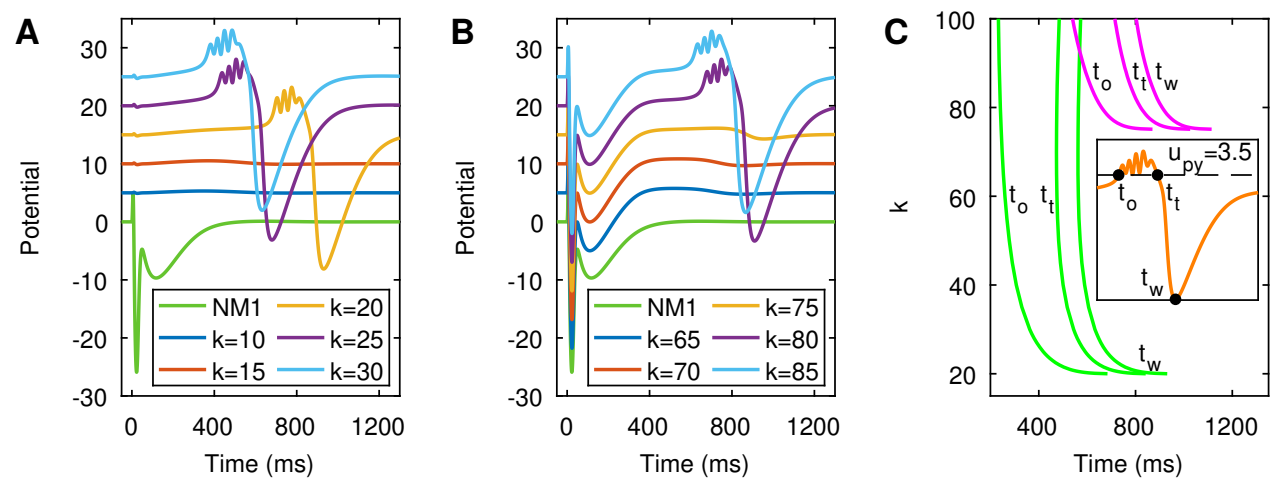

Figure 4.6: (A) Results for the configuration in Figure 4.3D. Neural mass 1 shows an early response (ER) upon stimulation (green line). Depending on the coupling strength $k$, the second neural mass shows a DR (other colours). (B) Results for the configuration in Figure 4.3E. Both neural masses show an ER. For sufficiently high values of the coupling strength $k$ also a DR is simulated on the second neural mass. (C) Latency of the simulated DRs against the coupling strength $k$ for the situation in $\mathbf{A}$ (green) and $\mathbf{B}$ (magenta) for three reference points. The inset shows the location of the three reference points on the wave shape of a DR.

only ERs are observed on the second neural mass.

Next, we study the influence of the connectivity strength $k$ on the latency of the DRs. For each DR, we consider three time points, i.e. the onset of the oscillations, $t_{o}$, the transition from oscillations to slow wave, $t_{t}$ and the time the slow wave reaches its minimum, $t_{s}$ (see inset of Figure 4.6C). Here, we defined $t_{o}$ and $t_{t}$ as the first and last time at which the response crosses $u_{p y}=3.5$. As is shown in Figure 4.6C, the latency of the DRs decreases rapidly for $k$ slightly above the threshold for which DRs emerge. If $k$ gets bigger, the time the DR shows oscillations grows as the $t_{o}$ remains decreasing for high $k$, while $t_{t}$ gets bigger if $k$ is above 60 (in case of a DR not preceded by an ER). The difference between $t_{t}$ and $t_{w}$ remains almost constant.

The model predicts that DRs which are preceded by an ER occur later than those which are not. We investigate this in the patient data. Figure 4.7A shows a histogram of the onset times of DRs for the two groups in a single patient confirming that DRs preceded by an ER occur in general later than those that are not. Histograms of the other patients are supplied in 4.S2. The boxplot in Figure 4.7B summarizes the results for all patients. Note that the timing of the simulated DRs without an ER is comparable to the data. DRs preceded by an ER occur rather late in the simulations in comparison with the data. Observe that in some patients a clear difference between the distributions is visible, while for others the median values are close together. A one-sided Wilcoxon rank-sum yields a significant $p$-value in 8 of the 11 patients for a significance level of 0.05 .

\subsubsection{A Mechanism for DRs}

So far we have shown that DRs can be modelled as indirect responses to SPES. We observed that DRs emerge all of a sudden, if the connectivity strength passes some critical value. The 

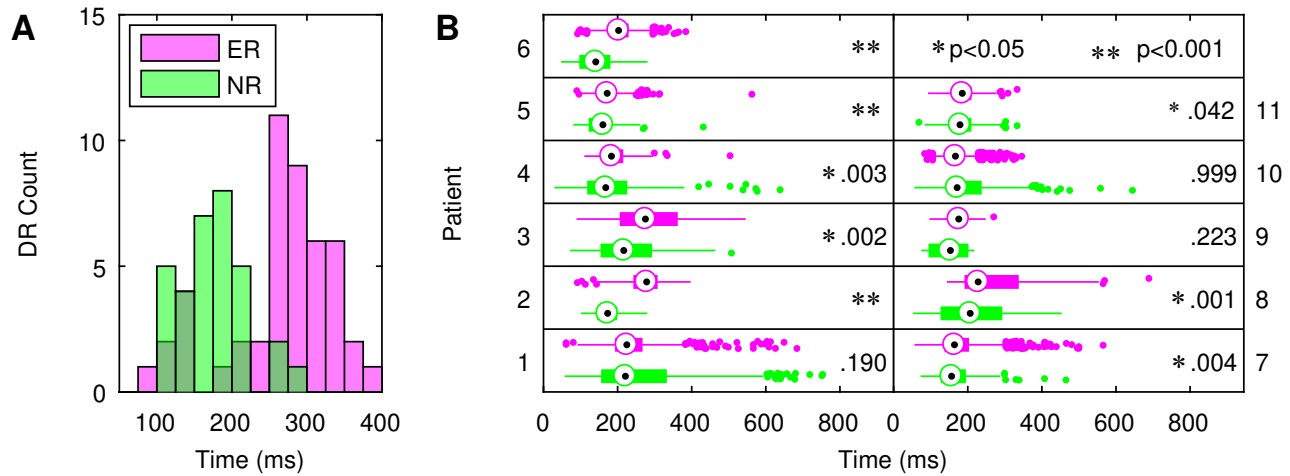

Figure 4.7: Onset time of delayed responses (DRs) in vivo. (A) Distribution of the onset time of DRs preceded by an ER (magenta) and DRs not preceded by an ER (green) in patient 2. (B) Boxplots for all patients showing the onset of the DRs, where colours refer to the same groups as in $\mathbf{A}$. Values at the right indicate $p$-values of the statistical test. Significant results (significance level 0.05) are marked with an asterisk. If $p<0.001$ results are marked with two asterisks.

next step is to understand the mechanism causing this sudden appearance. Using bifurcation analysis we study the influence of parameters on stationary and periodic solutions, i.e. equilibria and limit cycles, characterizing the activity of the neural mass.

For this analysis we focus solely on the second neural mass and treat the external input, $I$ as a parameter. Note that the input to this neural mass depends on both the connectivity strength $k$, and the activity of the first neural mass. So by studying the input as a bifurcation parameter we aim to get insight in the sudden appearance of DRs when the connectivity strength passes some critical value.
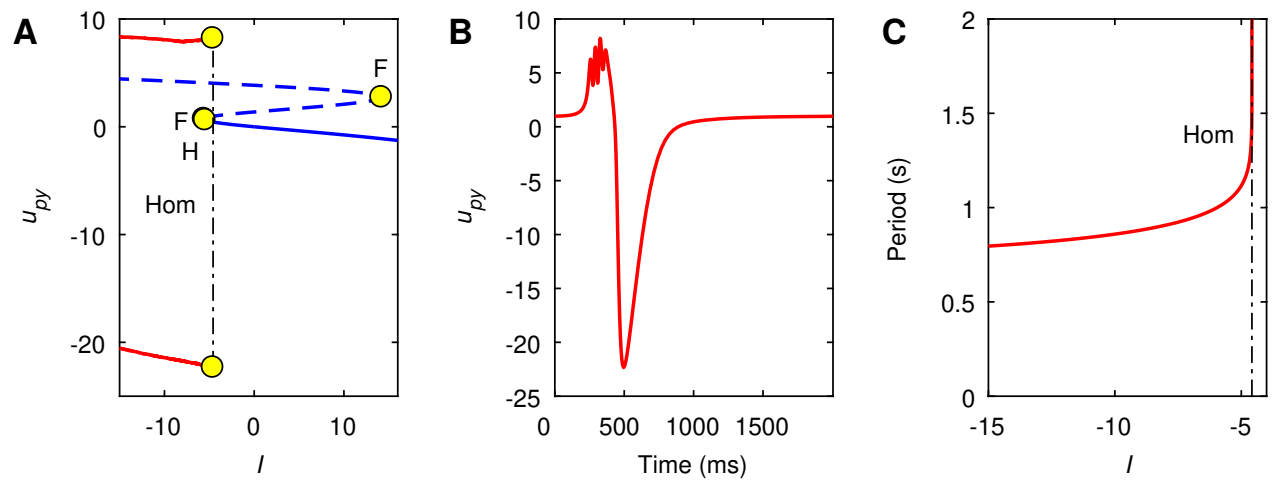

Figure 4.8: Bifurcation analysis. (A) Bifurcation diagram for varying external input $I$. Blue lines indicate equilibria, red lines are minima and maxima of limit cycles. Solid lines indicate stable solutions, while dashed lines indicate unstable ones. Fold $(\mathrm{F}), \operatorname{Hopf}(\mathrm{H})$ and homoclinic $(\mathrm{Hom})$ bifurcations are indicated by yellow circles. (B) Time profile of the stable limit cycle for $I=-7$. (C) Period of the stable limit cycle. The period approaches infinity near the homoclinic bifurcation. 
Results of the bifurcation analysis varying $I$ are shown in Figure 4.8A. Under normal circumstances the external input $I$, is zero. For this value the neural mass has an equilibrium (stationary solution) with $u_{p y}=0$. This equilibrium is stable, i.e. for any small perturbation of the equilibrium state, the neural mass will converge to the equilibrium if time proceeds. The solid blue line at the bottom of Figure 4.8A shows how this equilibrium behaves if $I$ is varied. For positive values of $I$ the equilibrium remains stable. For negative values of $I$ the equilibrium becomes unstable at $I \approx-5.46$, where it undergoes a Hopf bifurcation [134]. The unstable equilibrium curve, indicated by the dashed blue line in Figure 4.8A, contains two fold bifurcation at $I \approx-5.58$ and $I \approx 14.20$. At both these points the equilibrium curve changes direction, although the equilibrium remains unstable as it leaves the graph.

For $I \lesssim-4.58$ the neural mass has a stable limit cycle (periodic solution), with a waveform (Figure 4.8B) similar to that of the simulated DRs (Figure 4.6A, B). Like for the equilibrium, we continue the limit cycle varying $I$. The minimum and maximum amplitude of the limit cycle are indicated by the solid red lines in the bifurcation diagram. The limit cycle vanishes at $I \approx-4.58$ via a homoclinic bifurcation [134], which is characterized by an increase in the period of the limit cycle towards infinity (Figure 4.8C). In 4.S1 we discuss the bifurcation diagram in more detail.

From the bifurcation diagram we can deduce the mechanism responsible for generating DRs in the model. First, note that the input neural mass 2 receives from neural mass 1 showing an ER, is negative, as the average membrane potential of the pyramidal cells of neural mass 1 is negative. Therefore, neural mass 2 will follow the stable equilibrium branch for negative I. If $k$ is small, $I$ stays above the Hopf bifurcation at $I \approx-4.58$ and the neural mass will stay on the stable equilibrium branch while the input returns to zero. On the other hand, for larger $k, I$ will cross the Hopf bifurcation. As the stable equilibrium is not present here, the neural mass moves to the stable limit cycle. While the neural mass follows the limit cycle for approximately one period, the input $I$ returns to zero and crosses the homoclinic bifurcation where the limit cycle vanishes. As a consequence the neural mass returns to the equilibrium state.

\subsubsection{Stochastic DRs and Local Excitability}

We can use the mechanism described above to model DRs that have a stochastic appearance. Consider again two feedforward coupled neural masses as in Figure 4.3D and set the connectivity strength around the critical value for which DRs emerge, i.e. $k=20$. By adding noisy background input to the model, the second neural mass will pass the Hopf bifurcation occasionally and hence DRs will appear stochastically as show in Figure 4.9.

Next, we investigate how the probability of evoking a DR depends on both the connectivity strength $k$ and the intrinsic excitability of the second neural mass $C$. Figure 4.10 shows the result of simulating one hundred SPES stimulations of the model for various values of $C$ and $k$. Both increasing $k$ and $C$ increases the probability of evoking DRs. If $C$ gets too large the probability decreases slightly, because the neural mass also shows spontaneous interictal discharges in this case. The relation between $k$ and $C$ agrees with results found using bifurcation analysis varying $I$ and $C$ (see 4.S1). 


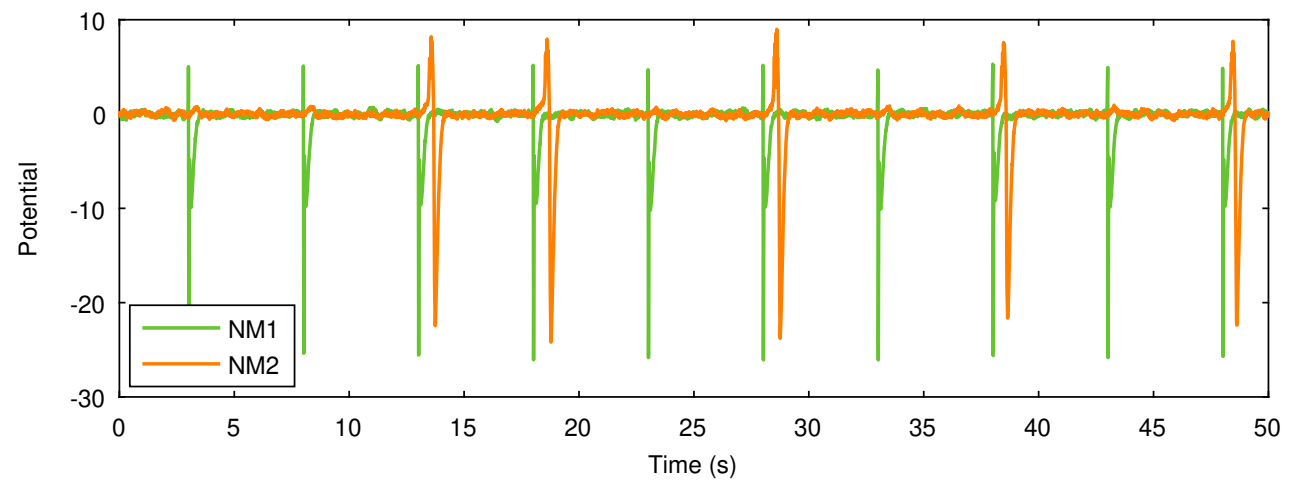

Figure 4.9: Simulating stochastic DRs. Simulation of ten stimulation trials for the configuration in Figure 4.3D where both neural masses receive additional background noise $(\sigma=1)$ for a coupling strength $k=20$. An ER appears consistently on neural mass 1, while a DR appears stochastically on the second neural mass.

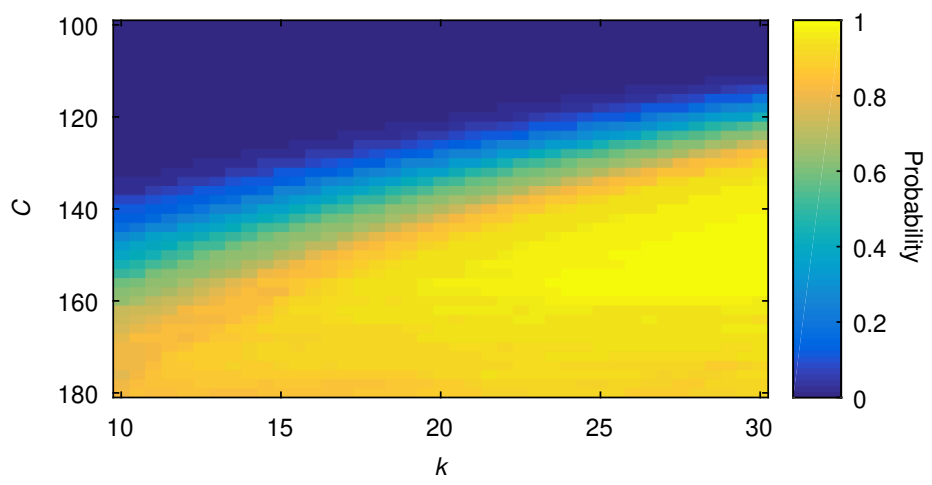

Figure 4.10: Probability of evoking delayed responses for varying $k$ and $C$. The connectivity strength $k$ and the intrinsic excitability $C$ are varied with steps of 0.5 and 2, respectively. For each combination of $k$ and $C 100$ stimulations were simulated with noise $(\sigma=1)$ and the number of evoked delayed responses was counted.

\subsection{Discussion}

With our data-driven modelling study, we have revealed how network connectivity and intrinsic excitability lead to pathological delayed responses upon stimulation. This sheds new light on their role as a clinical biomarker for epileptogenic tissue.

Starting with direct responses to stimulation, i.e. ERs, we showed that the two common peaks, i.e. the $\mathrm{N} 1$ and $\mathrm{N} 2$, can be explained by triggering not only the pyramidal cells but also both fast and slow inhibitory cells. This is in line with a modelling study by [31] using linear response theory. The NMM predicted an additional P0 peak which we subsequently identified in the data.

Next, based on connectivity analysis of ER networks [Chap. 3, 38], we concluded that DRs 
are indirect responses to the stimulation. Model simulations show that they may arise from feedforward propagation of ER-activity, and notably projections to inhibitory populations that normally restrain neural activity. Moreover, the model correctly predicted that DRs preceded by an ER occur later than DRs that are not. We find that DRs may appear when the input falls below a threshold, temporarily allowing the neural mass to enter a spiking state. This threshold also explains the stochastic appearance of DRs through noisy background activity. The probability for a DR to occur, depends on both the intrinsic excitability of a neural mass and the strength of the input from other regions with ERs. A higher intrinsic excitability moves this threshold closer to the normal state, so that less input is needed to pass the threshold, while stronger connections amplify input deviations to this region.

\subsubsection{Limitations of Modelling}

In this work we considered Wendling's neural mass that can generate several activity types as observed in ECoG signals $[53,57]$ and explains transitions in these activities seen during epileptic seizures [42]. However, an important feature which cannot be modelled is highfrequency activity. Model extensions with self-feedback for the fast inhibitory population $[55,135]$ allow to simulate responses with frequencies up to approximately $70 \mathrm{~Hz}$. Another study [130] considered a NMM consisting of only pyramidal and fast inhibitory cells to produce HFA. Their analysis shows that the connectivity constants in the loop between the fast inhibitory and pyramidal cells are important for the maximum frequency that the neural mass can generate. Adjusting these constants, the systems' activity will resonate with this frequency allowing frequencies up to $120 \mathrm{~Hz}$. Thus, it may be possible to simulate such high frequencies with a Wendling-type NMM extended with self-feedback for the fast inhibitory population, which may allow to increase the frequency of the fast oscillations at the start of the simulated DRs. Simulating higher frequencies with neural masses poses a challenge, as these models only take synaptic transmissions into account and not the fast electric transmissions via gap junctions which are, among other factors, hypothesized to play an important role in generation of fast-ripples $[42,136]$.

Following other neural mass studies $[52,53,56,59]$ we compare the average membrane potential of the pyramidal cells to measured EEG signals. As the pyramidal cells are the main contributor of EEG signals, due to the parallel alignment of their dendrites, their average membrane potential is a reasonable first approximation of the EEG signal. More specifically, the average membrane potential is a weighted sum of the postsynaptic potentials (PSPs) on the pyramidal cells. The currents induced by these PSPs are the main source of EEG signals [137]. The precise relation between the PSPs and the observed EEG however, depends on multiple factors, e.g. the relative location of the electrode with respect to the gyrus [137]. Therefore, differences in the waveforms of observed responses, such as the relative amplitude of the $\mathrm{N} 1$ and $\mathrm{N} 2$ responses, are not necessarily explained by different model parameters, but may originate from the precise measurement setup.

As a first step in understanding the mechanisms triggering early and delayed responses to SPES we investigated the most simple network configuration, i.e. we considered two feedforward coupled neural masses. These two neural masses model only a small part of neuronal tissue, while in reality also recurrent connections will be present. These factors will influence the precise waveforms of the ERs and especially the DRs. We think this could also explain the difference between the latency of DRs preceded by an ER in the model and 
in the data. Moreover, depending on the network topography, the connectivity strength and the intrinsic parameters of the neural masses, complex transient dynamics may arise [64]. However, within the current model the mechanisms responsible for the ERs (direct activation due to stimulation) and DRs (limit cycle) are robust with respect to small parameter changes.

We focused on modelling the two most common types of SPES responses, i.e. ERs and DRs. Besides these, two other responses have been observed, i.e. stable and repetitive responses [29, 30]. Stable responses are small, consistently appearing spikes or sharp waves, mostly superimposed on the N2 component of an ER [30]. They appear at the same electrodes for a variety of stimulation pairs [30], suggesting it to be a local phenomenon. Stable responses might therefore be modelled by slightly altering the parameters of a neural mass, or if needed, considering a small extension to the default neural mass. However, as stable responses are not related to the epileptic zone [30], such a change must not make the neural mass pathological.

Repetitive responses are observed exclusively in the frontal lobe and consist of an early response component which is repeated at least once [29]. They seem to be induced by stimulation of the epileptic zone and appear simultaneously in a spatially extended area [29]. The former suggests that it might be necessary to model the effect of the stimulation in detail. The latter might be explained by cortico-subcortical loops [29], which would require a spatially extended network of neural masses [64].

\subsubsection{ERs Reveal Feedforward Inhibition}

A crucial aspect in our model is that external input projects not only onto pyramidal cells but also to both slow and fast inhibitory populations. This is in line with the seminal work by [126] and has been considered in multiple computational modelling studies [58, 59, 127, 138-140]. These projections lead to direct activation of the inhibitory populations upon stimulation and as a consequence to the N1 and N2 peaks of ERs in the model. One might also consider external input projecting to the local excitatory cells of the neural mass. In 4.S1 we show that adding this connection does not quantitatively change our results, provided it is not too strong.

Single neuron measurements have revealed some distinct neuronal firing patterns after SPES, i.e. burst-suppression, burst only, suppression only or unchanged [141], where suppression typically lasts 5-10 times as long as bursting activity. In our model the different populations also show different firing patterns during an ER. Activity of the slow inhibitory cells is increased upon stimulation. Pyramidal cells and fast inhibitory cells first show increased activity followed by a long lasting period of decreased activity. The local excitatory population mainly shows a long lasting decrease in activity. It has also been suggested that only $\mathrm{N} 1$ peaks reflect direct activation of fibres, while the N2 might arise from cortico-cortical or cortico-subcortico-cortical reverberation circuits [34]. Our study shows that physiologically plausible values for the synaptic time constants, as reported in [130], can naturally explain the $\mathrm{N} 1$ and $\mathrm{N} 2$ peaks as a direct consequence of the stimulation.

Our model is able to reproduce common properties of ERs, like the linear relation between stimulation strength and the amplitude of ERs [95]. It has been reported that the majority of the waveforms contains either a N1 or N2 component, or both [31]. Therefore, we considered two types of ERs, those with only a N1 component and those with a N1 and N2 component. Simulations of ERs without N2 are easily achieved by weakening the projection to the slow inhibitory population. 


\subsubsection{Clinical Role of DRs}

Several studies have revealed the clinical value of DRs for supporting hypotheses when delineating the epileptogenic zone $[28,29,40]$. Our study provides a theoretical explanation for DRs and their use. DRs indicate that, already under normal conditions, the underlying neural mass is close to a state of epileptiform activity.

In our centre, DRs are visually classified based on spikes, ripples and fast-ripples using time-frequency images obtained from wavelet-analysis [40]. The majority of the DRs exhibits activity in at least the spike and ripple bands. The waveforms of the simulated DRs show a qualitative match, i.e. they consist of fast oscillations followed by a spike, although the frequency of the fast oscillations is below the ripple band. The morphology of DRs has similarities with interictal epileptiform discharges (IEDs). Moreover, [142] found that DRs were similar to at least one IED pattern for every patient, while in single neuron measurements similar firing patterns were found during IEDs and after SPES [141]. NMMs are also capable of simulating IEDs [124]. In fact, the mechanism responsible for these IEDs [57] is exactly the same as the mechanism responsible for DRs, i.e. both arise from temporarily escaping to the limit cycle corresponding to epileptiform activity. So, in patients with few spontaneous IEDs, SPES-triggered DRs may be a useful addition.

Computational modelling of epilepsy surgery has received considerable attention in recent years with promising results [Chap. 2, 65, 66, 67, 76, 119]. In this framework, neural masses or similar models are coupled through a patient-specific connectivity. Subsequently, the effect of surgery is predicted by removing nodes from the network and the results are compared against a base-line simulation of the model, as well as actual clinical outcomes. Typically for these studies, each node is equally excitable. Our finding that DRs depend on both local excitability and network effects suggests that both these factors play an important role in the ability of a node to start a seizure. Hence, SPES may improve the prediction of such frameworks in two ways: a patient-specific network can be obtained from ERs [Chap. 3, 38] and DRs can be used to differentiate excitability of the nodes.

\section{S1 Model Details \& Additional Bifurcation Analysis}

In this supplement we give a detailed description of the extended Wendling neural mass model. Next, we perform bifurcation analysis with respect to the input parameter $I$ in more detail and in a larger range than in the main text. Finally, we perform a two parameter bifurcation analysis in $C$ and $I$.

\section{S1.1 Detailed Model Description}

In this section we describe the system of differential equations governing the dynamics of the extended Wendling model. This model consists of four neuronal populations. Before we derive the equations for the full neural mass, we first discuss the general principle for a single population of a neural mass model.

Figure 4.11A shows an overview of a single population. Each population has a mean membrane potential $u$, acting as the state variable of the population. The mean membrane potential is influenced by external inputs, i.e. activity from other populations either inside or outside the same neural mass. The inputs to a population are average firing rates and can 
be either excitatory $\left(z_{e, 1}, \ldots, z_{e, N_{e}}\right)$ or inhibitory $\left(z_{i, 1}, \ldots, z_{i, N_{i}}\right)$. Average firing rates $z_{e, k}$ (or $z_{i, k}$ ) are converted to postsynaptic potentials (PSPs) $x_{e, k}\left(x_{i, k}\right)$ via a second-order linear differential equation:

$$
\ddot{x}(t)=Q q z(t)-2 q \dot{x}(t)-q^{2} x(t) .
$$

This differential equation is characterized by its impulse response function $h$ :

$$
h(t)= \begin{cases}Q q t e^{-q t} & t \geq 0, \\ 0 & t<0,\end{cases}
$$

with $1 / q$ the rising time of the synapse, i.e. the time after which the impulse response reaches its maximum, and $Q$ the synaptic gain, regulating the magnitude of $h$. We distinguish three types of PSP transformations: one excitatory, modeling inputs from pyramidal and excitatory cells, and two inhibitory ones, modeling input from fast and slow inhibitory populations. Impulse responses of these PSP transformations are shown in Figure 4.3B, while their parameter values are given in Table 4.1.

The mean membrane potential of a population is obtained by multiplying each PSP by a connectivity constant and then adding up all excitatory PSPs and subtracting all inhibitory ones, i.e.

$$
u=\sum_{k=1}^{N_{e}} C_{e, k} x_{e, k}-\sum_{k=1}^{N_{i}} C_{i, k} x_{i, k} .
$$

Finally, the activity of a population, serving as input for other populations, is determined via the firing function $S$. This sigmoidal function is given by:

$$
S(v)=\frac{2 e_{0}}{1+e^{r\left(v_{0}-v\right)}}-\frac{2 e_{0}}{1+e^{r v_{0}}} .
$$

A graph of this function is shown in Figure 4.3C. The parameters $r, e_{0}$, and $v_{0}$ influence the steepness, the magnitude, and the location of the median firing rate of the sigmoid function respectively. Their values are given in Table 4.1. Note that, in contrast to the original Wendling model [53], we shifted the sigmoid function such that $S(0)=0$ and hence, firing rates should be interpreted as deviations from baseline.

Differential equations for the extended Wendling neural mass are obtained by coupling four populations as shown in Figure 4.11B. For a neural mass $j$ we obtain the following set of differential equations:

$$
\begin{aligned}
& \ddot{x}_{1}^{(j)}=A a S\left(u_{p y}^{(j)}\right)-2 a \dot{x}_{1}^{(j)}-a^{2} x_{1}^{(j)}, \\
& \ddot{x}_{2}^{(j)}=A a S\left(u_{e x}^{(j)}\right)-2 a \dot{x}_{2}^{(j)}-a^{2} x_{2}^{(j)}, \\
& \ddot{x}_{3}^{(j)}=B b S\left(u_{i s}^{(j)}\right)-2 b \dot{x}_{3}^{(j)}-b^{2} x_{3}^{(j)}, \\
& \ddot{x}_{4}^{(j)}=G g S\left(u_{i f}^{(j)}\right)-2 g \dot{x}_{4}^{(j)}-g^{2} x_{4}^{(j)}, \\
& \ddot{x}_{5}^{(j)}=A a I^{(j)}-2 a \dot{x}_{5}^{(j)}-a^{2} x_{5}^{(j)} .
\end{aligned}
$$



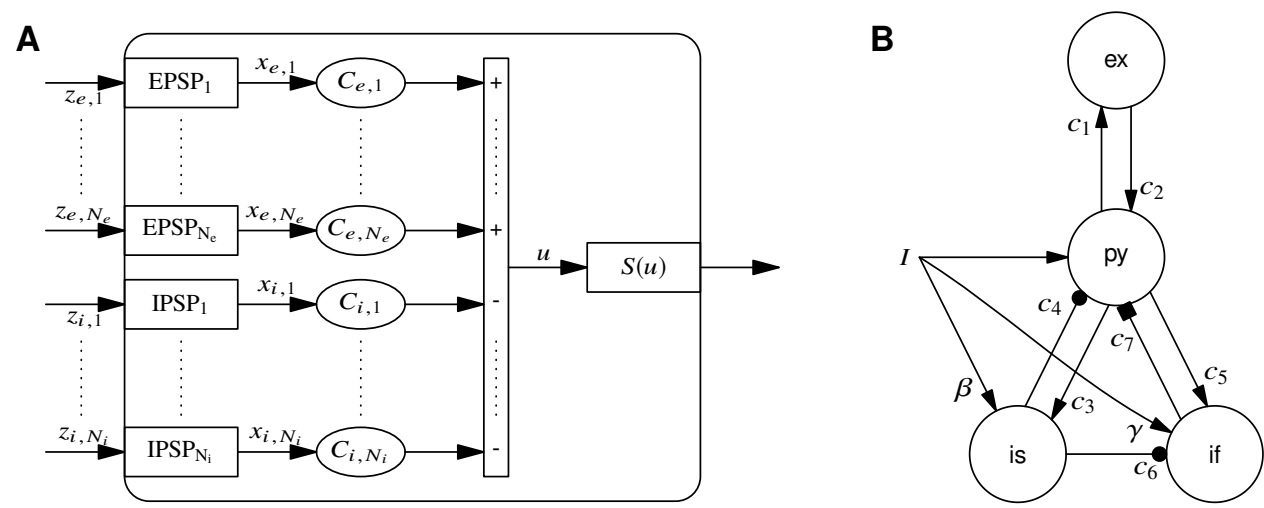

Figure 4.11: (A) Schematic overview of a single population. Each population may receive multiple excitatory inputs, labeled $z_{e, k}$, and inhibitory inputs, labeled $z_{i, k}$. These inputs are converted to the corresponding postsynaptic potentials $x_{e, k}$ and $x_{i, k}$. After multiplying by a connectivity constant excitatory PSPs are added and inhibitory ones subtracted resulting in the average membrane potential $u$. The activity of the population, serving as input for other populations, is obtained by applying a sigmoid function $S$ to $u$. (B) Architecture of a single neural mass. The circles represent the four populations, i.e. pyramidal cells (py), local excitatory cells (ex), slow inhibitory cells (is) and fast inhibitory cells (if). Arrows represent excitatory connections, circles and squares are slow and fast inhibitory connections. Labels along the connections indicate connection strengths.

The first four equations model the PSPs due to input from the pyramidal, excitatory, slow and fast inhibitory cells, respectively, while the last equation accounts for the external input $I^{(j)}$ to the neural mass. The potentials of the populations are given by:

$$
\begin{aligned}
& u_{p y}^{(j)}=C_{2}^{(j)} x_{2}^{(j)}-C_{4}^{(j)} x_{3}^{(j)}-C_{7}^{(j)} x_{4}^{(j)}+x_{5}^{(j)}, \\
& u_{e x}^{(j)}=C_{1}^{(j)} x_{1}^{(j)}, \\
& u_{i s}^{(j)}=C_{3}^{(j)} x_{1}^{(j)}+\beta x_{5}^{(j)}, \\
& u_{i f}^{(j)}=C_{5}^{(j)} x_{1}^{(j)}-C_{6}^{(j)} x_{3}^{(j)}+\gamma x_{5}^{(j)} .
\end{aligned}
$$

Constants $C_{l}^{(j)}$ denote the connectivity strengths, defined as $c_{l} C^{(j)}$, with $C^{(j)}$ an overall internal connectivity constant and $c_{l}$ a scaling factor. We set the constants $c_{l}$ to the same values as used by [53], see Table 4.1. In the first part we set $C^{(j)}$ to the same value as for standard Wendling model. For the bifurcation analysis we vary $C^{(j)}$ as it resembles the excitability of the neural mass. The factors $\beta$ and $\gamma$ in the equations for $u_{i s}$ and $u_{i f}$, are the fraction of the external input added to the slow and fast inhibitory cells, respectively.

The external input to a neural mass consists of three parts. First, the neural mass receives input from other neural masses. This input depends on the activity of the pyramidal cells and is given by:

$$
k \sum_{l=1}^{N} M_{j, l} S\left(u_{p y}^{(l)}\right),
$$

Here, $k$ is the overall connectivity strength and $M$ the coupling matrix, with $M_{j, l}$ the connection strength from neural mass $l$ to $j$. In this work, $M$ is a $2 \times 2$ matrix with $M_{2,1}=1$ and all other 


\begin{tabular}{|l|l|l|}
\hline Param. & Description & Value \\
\hline$A$ & Excitatory synaptic gain & $4.5 \mathrm{mV}$ \\
$B$ & Slow inhibitory synaptic gain & $7 \mathrm{mV}$ \\
$G$ & Fast inhibitory synaptic gain & $25 \mathrm{mV}$ \\
\hline$a$ & Reciprocal of excitatory time constant & $100 \mathrm{~s}^{-1}$ \\
$b$ & Reciprocal of slow inhibitory time constant & $10 \mathrm{~s}^{-1}$ \\
$g$ & Reciprocal of fast inhibitory time constant & $300 \mathrm{~s}^{-1}$ \\
\hline$C$ & Internal connectivity constant & 135 \\
$c_{1}$ & Relative conn. strength pyramidal to excitatory cells & 1 \\
$c_{2}$ & Relative conn. strength excitatory to pyramidal cells & 0.8 \\
$c_{3}$ & Relative conn. strength pyramidal to slow inhibitory cells & 0.25 \\
$c_{4}$ & Relative conn. strength slow inhibitory to pyramidal cells & 0.25 \\
$c_{5}$ & Relative conn. strength pyramidal to fast inhibitory cells & 0.3 \\
$c_{6}$ & Relative conn. strength slow to fast inhibitory cells & 0.1 \\
$c_{7}$ & Relative conn. strength fast inhibitory to pyramidal cells & 0.8 \\
\hline$\sigma$ & Standard deviation of noise & $0 \mathrm{or}^{-1} \mathrm{~s}^{-1}$ \\
$k$ & Coupling strength between neural masses & varied \\
$\beta$ & Scaling constant for external input to slow inhibitory cells & 1 \\
$\gamma$ & Scaling constant for external input to fast inhibitory cells & 0.7 \\
\hline$e_{0}$ & Half of difference between min and max of the sigmoid function & $2.5 \mathrm{~s}^{-1}$ \\
$v_{0}$ & Potential for which the sigmoid function has its median value & $4.5 \mathrm{mV}^{-1}$ \\
$r$ & Steepness of sigmoid function & $0.56 \mathrm{mV}^{-1}$ \\
\hline
\end{tabular}

Table 4.1: Parameters for the neural mass model and their default values.

entries 0 , as we only consider two feed-forward coupled neural masses. Second, the neural mass receives background input modeled as zero-mean Gaussian white noise with standard deviation $\sigma$. Finally, a neural mass may receive input due to single pulse electrical stimulation (SPES), which is modeled as a block pulse with an amplitude $P_{0}=1500$ and a duration of $5 \mathrm{~ms}$. As explained in the main text multiple neural masses can receive this input at the same time as we assume that SPES activates the outgoing fibers of a stimulated region [33, 132].

In total we have a system of ten second-order differential equations. Solutions to these Itô-type stochastic differential equations are computed using an Euler-Maruyama scheme with fixed time steps of 0.1 milliseconds.

\section{S1.2 The effect of adding input to excitatory cells}

External input in the model projects to the pyramidal cells as well as the two inhibitory populations. Here we investigate the influence of additional input to the local excitatory cells. Similarly as for the two inhibitory populations, we project the external input to the neural mass also onto the excitatory interneuron population in a proportional manner using a scaling constant $\alpha$. Figure 4.12A shows the effect of increasing $\alpha$ on the ER. If the projection to the excitatory population is not too strong, i.e. $\alpha \leq 0.6$, the change in waveform of the early response is minimal. Only the amplitudes of the N1 and P1 peaks are affected, in a linear fashion. For $\alpha>0.6$, nonlinear effects come into play, which can be counterbalanced by 
increasing values for the internal inhibitory coupling (not shown). Next, the effect of $\alpha$ on the DRs is shown in Figures 4.12B and C for $k=30$ and $k=100$. For $k=30$ a DR is evoked for $\alpha \leq 0.2$. By increasing the coupling strength $k$ DRs can also be found for $\alpha \leq 0.4$. These simulations show that adding feedforward input to the local excitatory cells does not quantitatively change our results, provided it is not too strong. Therefore, we ignore the feedforward excitation in the main text.
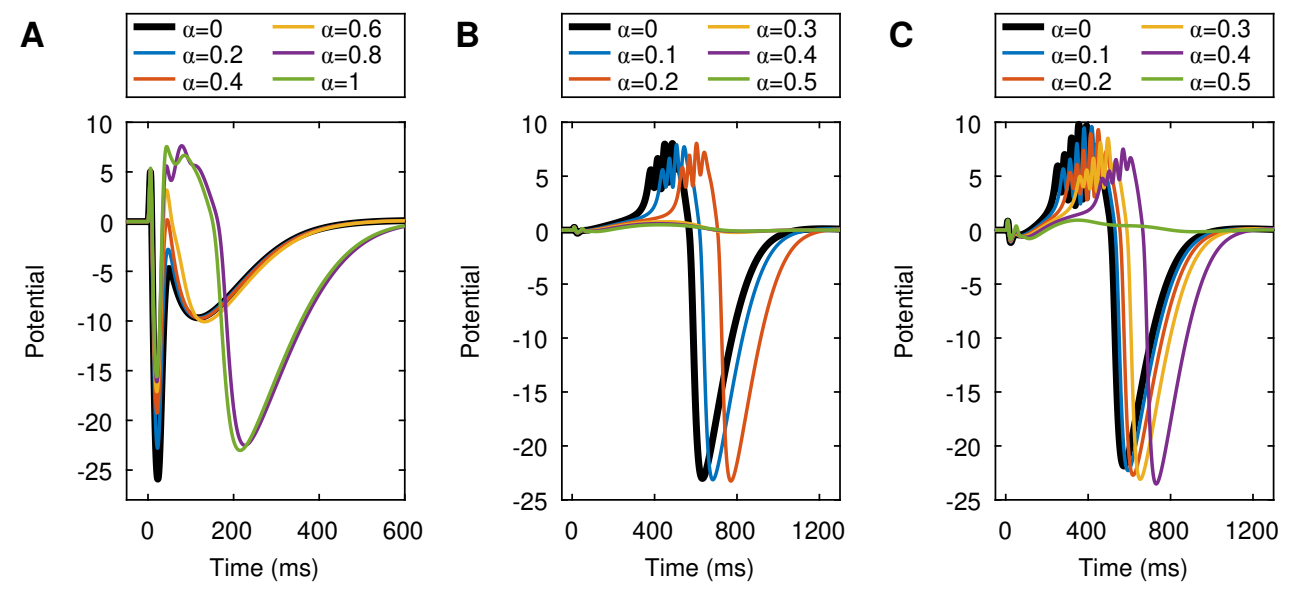

Figure 4.12: Effect of $\alpha$ on simulated responses. (A) Simulated ERs for various values of $\alpha$. (B, C) Simulated DRs for $k=30$ and $k=100$, respectively.

\section{S1.3 One Parameter Bifurcation Diagram}

In the main text we show the relevant bifurcations for modeling delayed responses (DRs), here we study the bifurcation diagram varying $I$ in more detail. Bifurcation analysis is performed numerically using MatCont [133]. The resulting bifurcation diagram is shown in Figure 4.13A. Starting from the stable equilibrium at $I=0$ and continuing the equilibrium curve for negative values of $I$ we find a Hopf bifurcation [134] at $I \approx-5.46$, two fold bifurcations [134] at $I \approx 5.58$ and $I=14.2$ respectively, and another Hopf bifurcation at $I \approx-24.05$. Between the two Hopf bifurcations the equilibrium is unstable, with an unstable manifold of dimension one between the two fold bifurcations and two otherwise. Both for large positive and negative values of $I$ the equilibrium undergoes two additional fold bifurcations (not shown).

From the first Hopf bifurcation an unstable limit cycle emerges. This limit cycle exists only in a small range of $I$ values and vanishes via a saddle homoclinic bifurcation (see [134]). More interesting is the unstable limit cycle emerging from the second Hopf bifurcation, which starts as an oscillation with a frequency of about $5 \mathrm{~Hz}$ (Figure 4.13D). Around $I=44.6$ this limit cycle undergoes several fold of cycles bifurcations (see Figure 4.13B), while its minimum decreases rapidly. After the last fold of cycles bifurcation the limit cycle is stable and has also reached the characteristic form of a DR, i.e. some fast oscillations followed by the slow wave, with a period of around $0.75 \mathrm{~s}$. This shape persists along the stable branch of the 

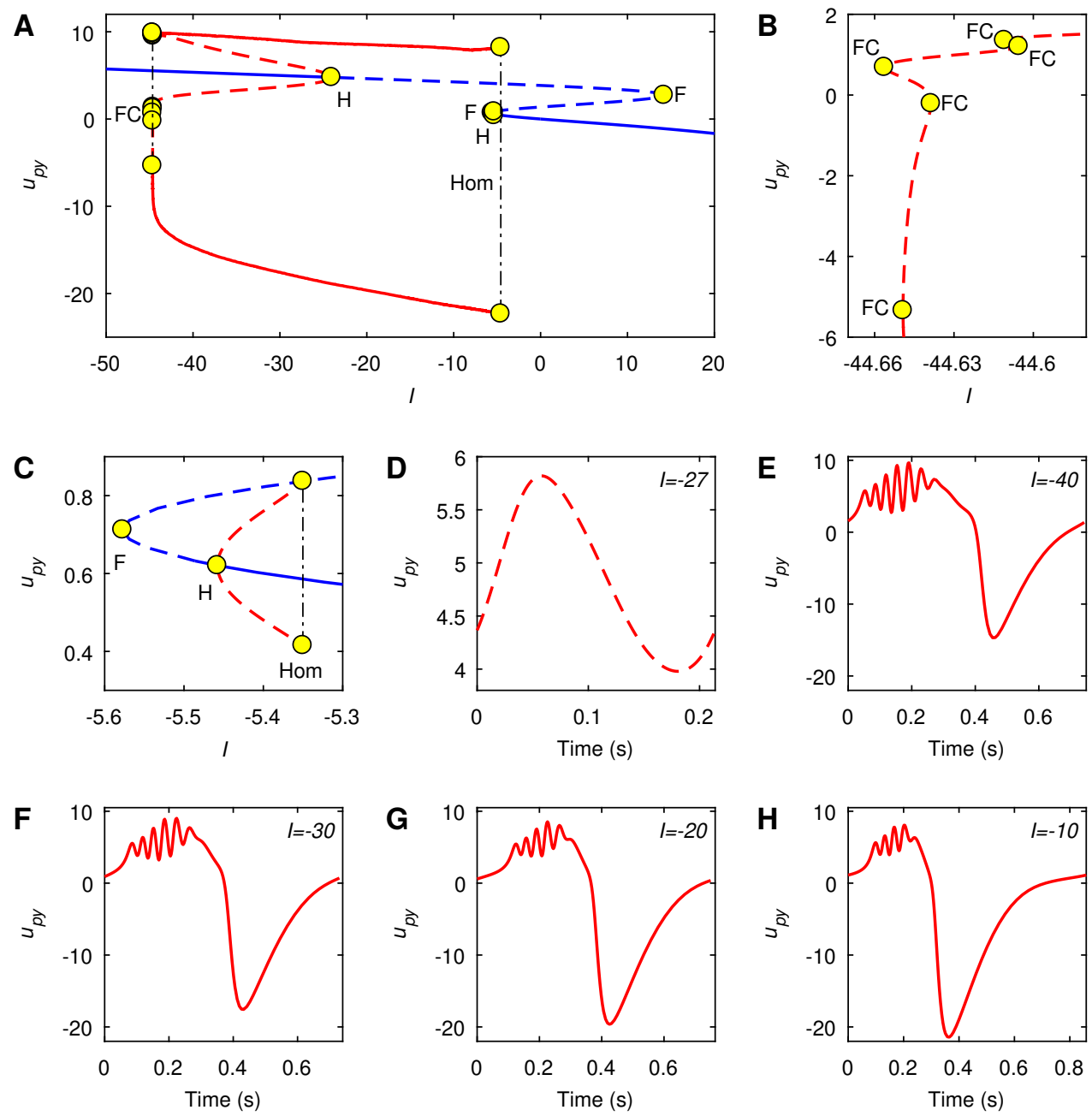

Figure 4.13: (A) Extended bifurcation diagram. Blue and red lines indicate equilibria and, minimum and maximum of limit cycles respectively. Solid lines and dashed lines represent stable and unstable solutions respectively. Fold (F), Hopf (H), fold of cycles (FC) and, saddle homoclinic (Hom) bifurcations are highlighted by yellow circles. (B) Close-up of $\mathbf{A}$ showing the minimum of the limit cycle around the fold of cycle bifurcations. (C) Magnification of $\mathbf{A}$ around the Hopf and fold bifurcations occurring near $I=5$. (D-H) Time profiles of the limit cycle. D shows the unstable limit cycle at $I=-27$, shortly after the Hopf bifurcation. E-H show the stable limit cycle for $I=-40,-30,-20$ and -10 , respectively.

limit cycle curve (see Figure 4.13D). The period stays approximately constant till $I \approx 10$ and then increases rapidly. Finally, the limit cycle disappears via a saddle homoclinic bifurcation near $I \approx-4.58$. Note that there is bistability, i.e. both the stable limit cycle and the stable equilibrium exist, for $I$ between -5.46 and -4.58 . 


\section{S1.4 Two Parameter Bifurcation Diagram}

So far we performed bifurcation analysis in one parameter, i.e. I, which revealed fold, Hopf and saddle homoclinic bifurcations. As discussed in the main text the first Hopf bifurcation is important as here the equilibrium loses stability. The same holds for the homoclinic bifurcation where the stable limit cycle vanishes. We now study how these bifurcation points changes in both $I$ and $C$.

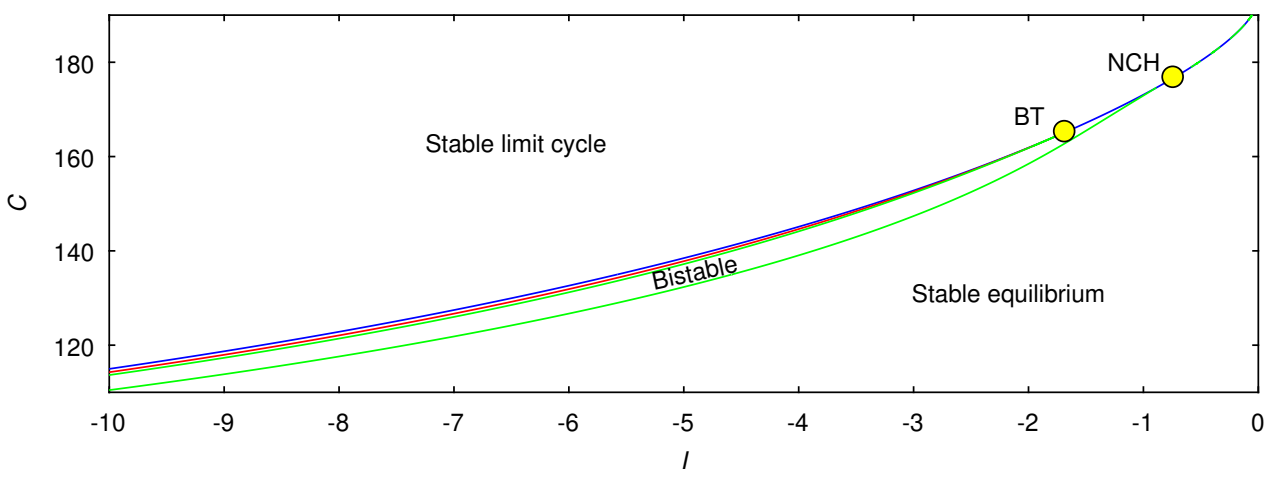

Figure 4.14: Bifurcation diagram in $I$ and $C$. Blue line: fold bifurcation, red line: Hopf bifurcation, green lines: homoclinic bifurcation, dashed blue-green line: saddle-node homoclinic bifurcation.

Figure 4.14 shows the bifurcation diagram in $I$ and $C$. The curves in this diagram show how the fold, Hopf, and homoclinic bifurcations vary with $I$ and $C$. Four lines pass through $C=135$, which represent from left to right the fold bifurcation (blue), Hopf bifurcation (red), homoclinic bifurcation of the unstable limit cycle (green) and the homoclinic bifurcation of the stable limit cycle (also green). To the right of the Hopf curve a stable equilibrium exists, while the stable limit cycle is present to the left of the right homoclinic curve. Note that there is a small area in which the system is bistable, i.e. both a stable equilibrium and limit cycle exist.

At $I \approx-1.68$ and $C \approx 165.2$ a Bogdanov-Takens bifurcation occurs and the Hopf and homoclinic curves terminate at the fold curve [134]. For larger values of $C$, the equilibrium loses stability at the fold curve. The fold curve and the remaining saddle homoclinic curve meet at the non-central homoclinic saddle-node bifurcation $(\mathrm{NCH})$ located at $I \approx-0.74$ and $C \approx 176.7$ [143]. Here, the saddle homoclinic curve ends and the fold curve turns into a saddle-node homoclinic curve [134] (dashed blue-green line). At the saddle-node homoclinic curve the limit cycle vanishes and simultaneously the equilibrium loses stability.

From the bifurcation diagram it follows that the proposed mechanism underlying DRs is robust with respect to $C$. If the input $I$ is temporarily lowered enough then the stable equilibrium loses stability via either a Hopf, fold or saddle-node homoclinic bifurcation and the neural mass follows the stable limit cycle for about one period. While the input returns to zero the stable limit cycle vanishes via a homoclinic bifurcation (either a saddle homoclinic or a saddle-node homoclinic) and the neural mass will return to the equilibrium state. Moreover, for a higher intrinsic excitability $C$, these bifurcations occur for values of $I$ closer to zero, implying that DRs can be generated using a smaller input deviation. As the 
deviation of the input increases with the connectivity strength $k$, this notion agrees with the results in Figure 4.10, which shows that DRs occur for a smaller connectivity strength $k$ if the excitability is higher.

\section{S2 DR Onset Times in Other Patients}

In this supplement we show the histograms with the distribution of the onset time of DRs for patients 1 and 3-11 (see Figure 4.15 ). Onset times of DRs is significantly later for DRs preceded by an ER compared to those that are not for patients $2-8$ and 11 , although the distributions have a large overlap in most patients.
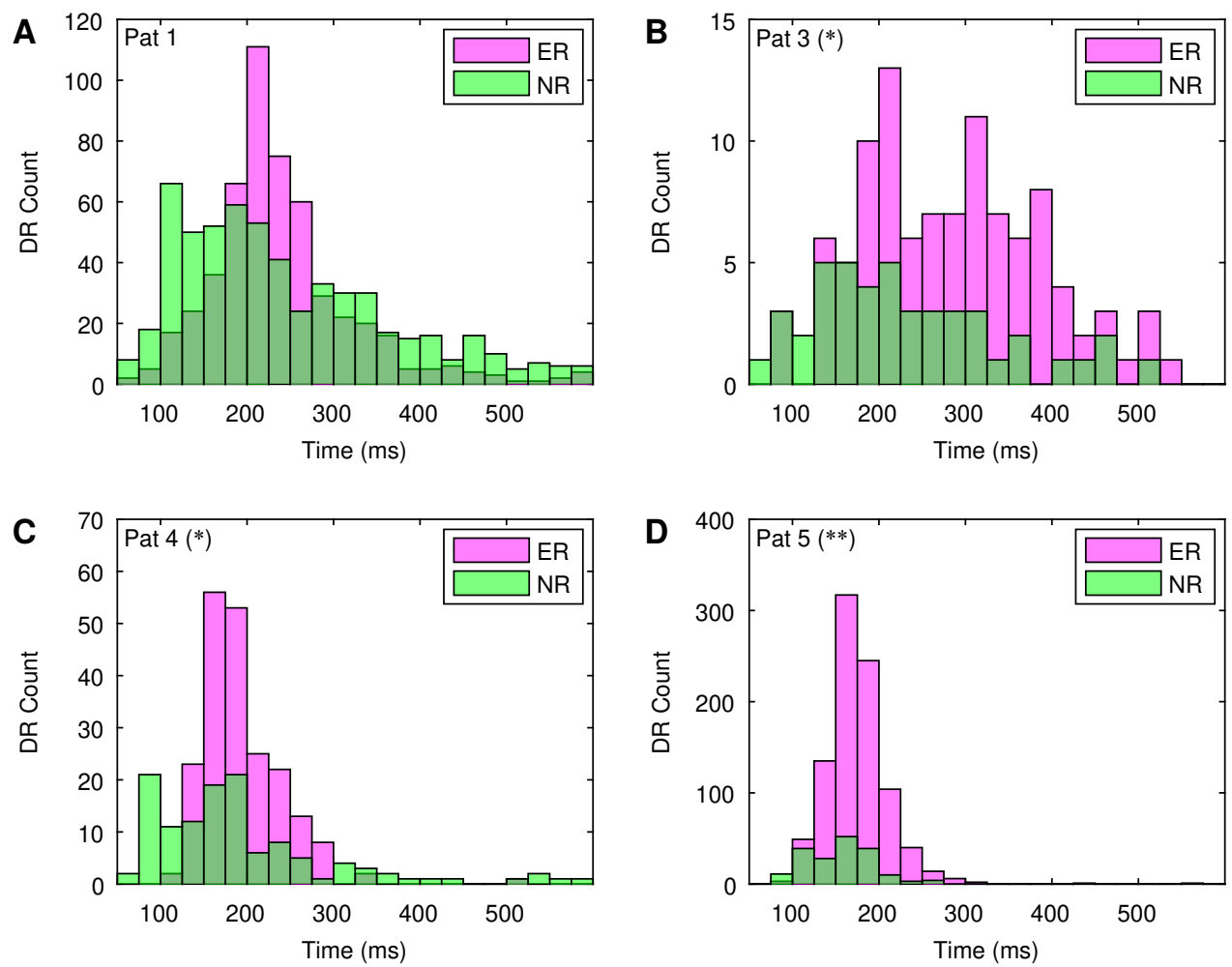

Figure 4.15: (continues on next page) 

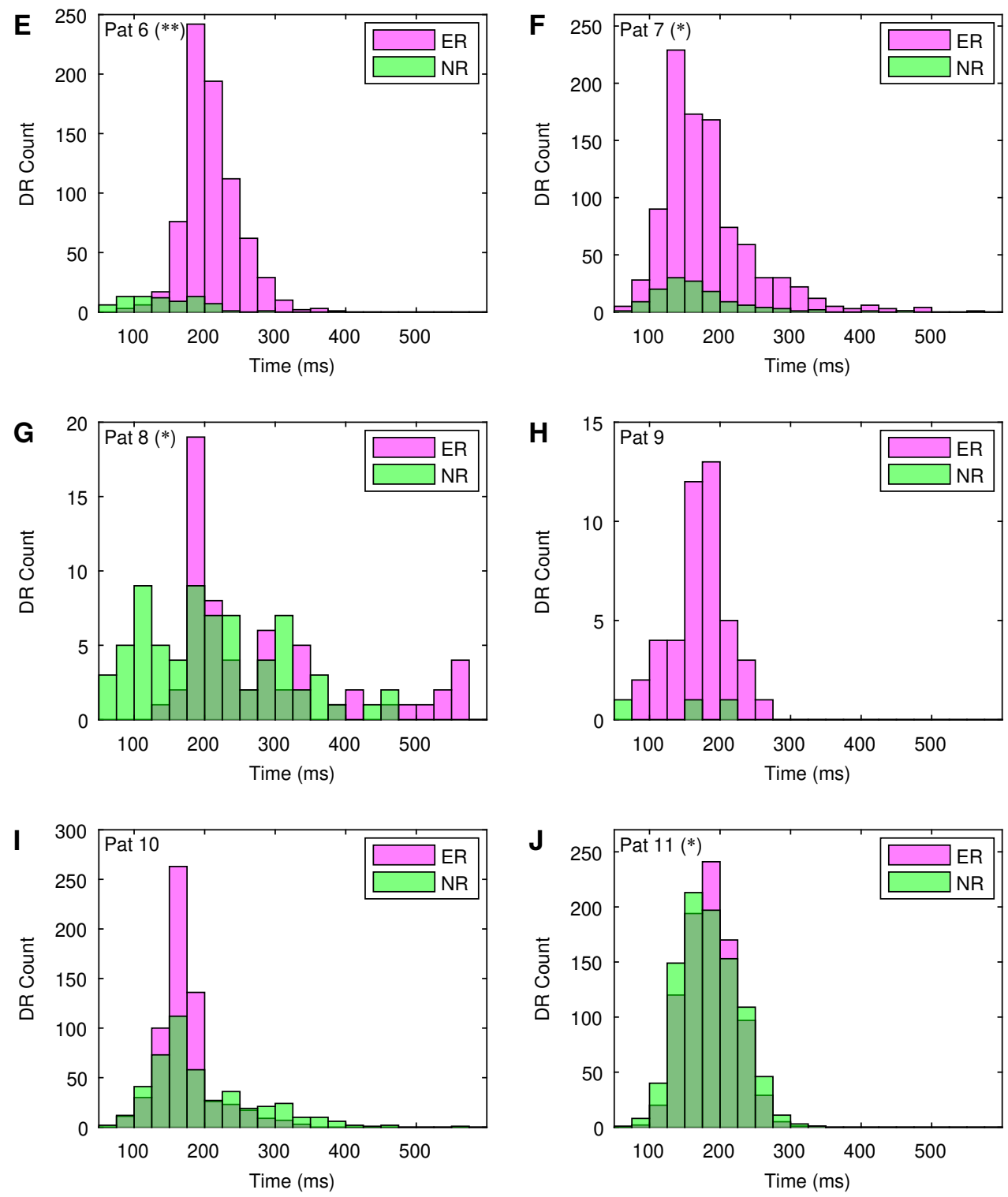

Figure 4.15: Distribution of onset times of delayed responses preceded by an ER (magenta) and delayed responses not preceded by an ER (green) for patients 1 and 3-11. Statistical significance results are indicated by $(*)$ and $(* *)$ for $p<0.05$ and $p<0.001$, respectively. 


\section{Chapter 5}

\section{On Analysis of Inputs Triggering Large Nonlinear Neural Responses}

Slow-fast dynamics in the Wendling neural mass model

Jurgen Hebbink, Stephan van Gils, Hil Meijer 


\section{Abstract}

Many applications in neuroscience, such as electrical and magnetic stimulation, can be modelled as short transient input to non-linear dynamical systems. In excitable systems, small input yields more or less linear responses, while for increasing stimulation strength large non-linear responses may show up suddenly. A challenging task is to determine the transition between the two different response types.

In this chapter we consider such a transition between normal and pathological responses in a coupled Wendling neural masses model as we encountered in a previous study. The different timescales of inhibition in this model allow a slow-fast analysis of the systems' response. The two response types are separated by a high-dimensional stable manifold of a saddle slow manifold. Large pathological responses appear if the fast subsystem escapes from this manifold to another attractor. The typical fast oscillations seen during the pathological responses are explained by the bifurcation diagram of the fast subsystem. Under normal conditions these oscillations are suppressed by slow inhibition. External stimulation temporarily releases the fast subsystem from this slow inhibition. The critical response can be formulated as a boundary value problem with one free parameter and can be used to study the dependency of the transition between the two response types upon the system parameters. 


\subsection{Introduction}

Invasive stimulation of the human brain in the form of electrical or magnetic pulses can be used to explore properties of the neurophysiological, or pathological, system. In mathematical models such pulses are short transient inputs to a non-linear dynamical system. For a small perturbation, the response can be studied using linear response theory. In excitable systems, increasing stimulus strength may suddenly induce large nonlinear phenomena in, for instance, transcranial magnetic stimulation [144, 145] or single pulse electrical stimulation (SPES), $[28,146]$. Characterizing the threshold between linear and non-linear responses remains a challenge in most applications.

Many systems, including those from neurosience, involve processes acting at different timescales, allowing a slow-fast analysis. The dynamics of the fast subsystem can be studied using bifurcation analysis with the slow variables as parameters. The attractors of the fast subsystem, e.g. stable equilibria and limit cycles, define slow manifolds. Bifurcations like fold points, indicate where properties of these manifolds change. Solutions of the full system will move on these slow manifolds. When reaching the boundary of such a manifold, orbits jump to another (part of the) attracting slow manifold. In many studies, the slow variables vary autonomously based on the attractor of the fast subsystem, e.g. bursters [47, 147-149]. This analysis can also be applied to cases where the system receives slow external input.

An example arises in modelling pathological responses to single pulse electrical stimulation (SPES). SPES is a technique for probing epileptogenicity in the presurgical work-up for epilepsy surgery $[28,40]$. During SPES, brief electrical pulses are given to the cortex evoking early responses (ERs) and delayed responses (DRs). Early responses are direct responses to the stimulation indicating brain connectivity [Chap. 3, 33, 34]. As the amplitude of ERs scales linearly with the stimulation strength [95], they can be modelled as (approximately) linear responses [Chap. 4, 31]. Delayed responses are associated with epileptogenic cortex and appear in an all-or-nothing fashion [28, 40].

In chapter 4 we showed that DRs can be modelled as indirect responses to SPES using two feed-forward coupled Wendling neural masses [53]. The short stimulus evokes activity at the first neural mass resulting in a slow input to the second neural mass. Depending on the connectivity strength, the second neural mass shows either a small normal response or a large, pathological DR-like response. We identified a saddle-node bifurcation in the full system as the transition, but a closer examination reveals a more subtle mechanism. This is the main focus of this manuscript. In addition, we will explain the waveform of the DR, which exhibits some fast oscillations followed by a large slow wave, using slow-fast analysis of the Wendling neural mass model.

Another previous study [150] also performed a slow-fast analysis of the Wendling neural mass model. With an additional slowly varying parameter, the authors made a first attempt to investigate dynamic transitions in activity seen in EEG, e.g. during seizures. This revealed a torus canard, which was closely related to the bifurcation structure of the original model. In contrast, our slow-fast analysis exploits the different timescales of inhibition in the Wendling neural mass. Together with a slowly varying parameter this yields a two dimensional slow space, similar to [149], but with driving external input.

Our manuscript is organized as follows. First we introduce the equations of the Wendling neural mass model and show simulated ERs and DRs. Next, we indicate slow and fast variables and perform bifurcation analysis of the fast system with the slow variable and the input as 
parameters. By combining the simulations with the bifurcation diagram we investigate the transition between the responses and the shape of the simulated DRs. Finally, we formulate a boundary value problem for the critical response, which separates the two different responses.

\subsection{Material and Methods}

\subsubsection{Model}

Neural mass models mimic the activity of large groups of neurons, typically covering a couple of millimeters up to a few centimeters of cortex. In this chapter we consider Wendlings' neural mass [53]. Here we give a brief description of this model, for details see [Chap. 4, 53].

The Wendling neural mass describes the activity of four populations, i.e. pyramidal cells, and excitatory, fast and slow inhibitory interneurons. These populations are coupled as indicated in Figure 5.1A. For each population we describe the dynamics of the mean membrane potential. The activity or firing rate of the population depends on its mean membrane potential and is given by a sigmoid function:

$$
S(v)=\frac{2 e_{0}}{1+e^{r\left(v_{0}-v\right)}}-\frac{2 e_{0}}{1+e^{r v_{0}}} .
$$
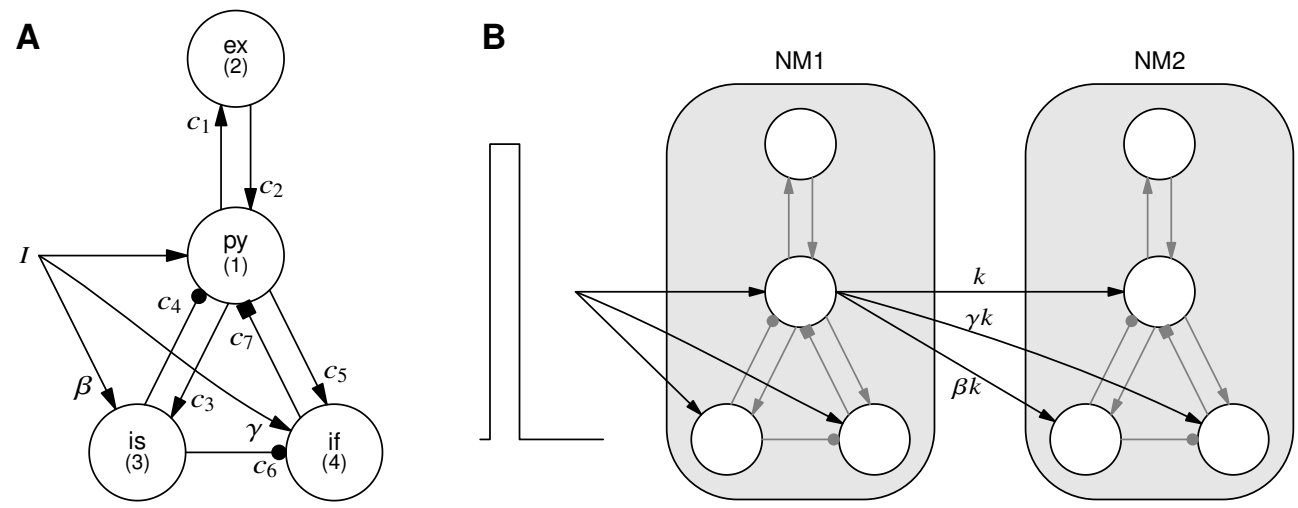

Figure 5.1: Model overview. (A) Schematic layout of the Wendling model with pyramidal cells (py), excitatory (ex) and fast (if) and slow (is) inhibitory interneurons. Arrows indicate excitatory connections, while dots/square represent inhibitory connections. (B) Two feed-forward coupled Wendling neural masses of which the first receives a block pulse as input to model SPES responses.

Each population has synaptic output based on its activity $z$ generating a post synaptic potential (PSP) $x$ at the populations it projects to. This process is modelled by the following linear, second order differential equation:

$$
\ddot{x}(t)=Q q z(t)-2 q \dot{x}(t)-q^{2} x(t),
$$

where $Q$ is the synaptic gain and $1 / q$ is the time constant. We consider three types of synapses, i.e. fast and slow inhibitory and fast excitatory. The mean membrane potential of a population is obtained by multiplying each PSP by a connectivity constant and then adding 
up its excitatory PSPs and subtracting the inhibitory ones. For a single neural mass we have the following system of differential equations:

$$
\begin{array}{ll}
\dot{x}_{1}=y_{1}, & \dot{y}_{1}=\operatorname{AaS}\left(u_{p y}\right)-2 a y_{1}-a^{2} x_{1}, \\
\dot{x}_{2}=y_{2}, & \dot{y}_{2}=\operatorname{AaS}\left(u_{e x}\right)-2 a y_{2}-a^{2} x_{2}, \\
\dot{x}_{3}=y_{3}, & \dot{y}_{3}=B b S\left(u_{i s}\right)-2 b y_{3}-b^{2} x_{3}, \\
\dot{x}_{4}=y_{4}, & \dot{y}_{4}=G g S\left(u_{i f}\right)-2 g y_{4}-g^{2} x_{4},
\end{array}
$$

modelling the PSPs induced by pyramidal, excitatory, slow and fast inhibitory cells, respectively. The mean membrane potentials of the populations are given by:

$$
\begin{aligned}
u_{p y} & =c_{2} C x_{2}-c_{4} C x_{3}-c_{7} C x_{4}+x_{5}, \\
u_{e x} & =c_{1} C x_{1}, \\
u_{i s} & =c_{3} C x_{1}+\beta x_{5}, \\
u_{i f} & =c_{5} C x_{1}-c_{6} C x_{3}+\gamma x_{5},
\end{aligned}
$$

with $C$ a global internal connectivity constant. The function $x_{5}(t)$ accounts for the PSP arising from external input to the neural mass and is commonly prescribed as an excitatory PSP transformation of some pulse rate function $I(t)$, i.e.

$$
\dot{x}_{5}=y_{5}, \quad \dot{y}_{5}=A a I-2 a y_{5}-a^{2} x_{5} .
$$

In addition to the typical input to the pyramidal cells as in [53], the external input projects also to the inhibitory populations. All parameter values are given in Table 4.1. Usually, the potential of the pyramidal cells is studied as model output as this relates to clinically measured EEG signals [125].

\subsubsection{Simulated Responses}

Responses to SPES can be modeled using two feed-forward coupled neural masses as shown in Figure 5.1B. Here, the first neural mass (NM1) receives external input in the form of a block pulse (amplitude $1500 \mathrm{~s}^{-1}$, duration $5 \mathrm{~ms}$ ), modelling the effect of SPES. The firing rate of the pyramidal cells of the first neural mass multiplied by a connectivity strength $k$ serves as input for NM2. In chapter 4 we showed that upon stimulation NM1 has a characteristic early response (ER), see Figure 5.2A, and for sufficiently high $k$ NM2 shows a delayed response (DR), see Figure 5.2B.

To understand the effect of the stimulation on NM2, we first explain ERs in more detail. ERs can be viewed as direct, linear responses to stimulation [31]. As observed in clinical recordings, a simulated ER consists of a positive peak succeeded by two negative peaks. The positive peak resembles direct excitation due to the stimulation. The two negative peaks arise from input from the fast and slow inhibitory populations, respectively (see ch. 4), which are also activated directly by the stimulation. The firing rate of the pyramidal cells (Figure 5.2C) is, apart from a short time interval directly after the stimulation, below its baseline level for about $400 \mathrm{~ms}$.

Next, the external input to the second population, $x_{5}^{(2)}$, is obtained by the excitatory PSP transform. This is given by eq. (5.4) where we take for $I$ the input $k S\left(u_{p y}^{(1)}\right)$, with $k$ the 

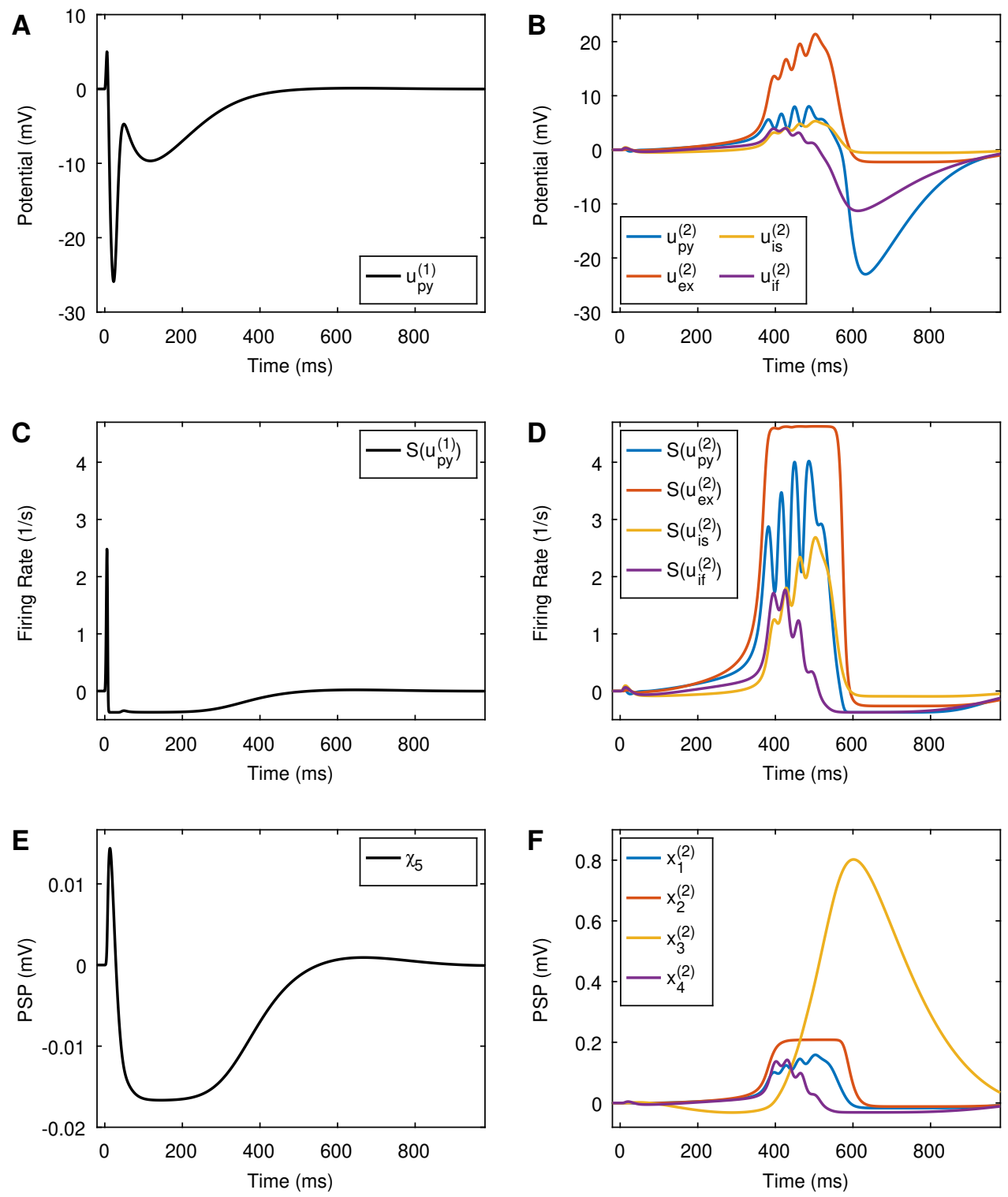

Figure 5.2: Simulated early and delayed responses. (A, C) Potential and firing rate of the pyramidal cells of NM1 exhibiting an ER. (E) PSP of the external input from NM1 to NM2. (B, D, F) Potential, firing rate and PSPs of NM2, respectively. 
connectivity strength between NM1 and NM2. As the PSP transformation is linear, we can write $x_{5}(t)=k \chi_{5}(t)$, with $\chi_{5}(t)$ the PSP transform (5.4) of $S\left(u_{p y}^{(1)}\right)$. Figure 5.2E shows $\chi_{5}$. We note that $\chi_{5}$ does not depend on $k$. In the following we will study the response of NM2 to the stimulation and the influence of $k$ on this response assuming that $\chi_{5}(t)$ is some (numerically) known function.

The mean potential of each population of the second neural mass for $k=30$ is shown in Figure 5.2B. This $k$ value is sufficiently high to evoke a DR. Simulated DRs consist of some fast oscillations with a frequency of around $30 \mathrm{~Hz}$ followed by a slow wave. The PSPs associated with the second neural mass are shown in Figure 5.2F. Note that $x_{3}$, i.e. the PSP induced by the slow inhibitory population, varies slowly compared to those of the other populations.

\subsection{Results}

\subsubsection{Slow-Fast Analysis}

A feature of the Wendling neural mass are the two inhibitory populations each with its own timescale. Transmission via fast inhibitory and excitatory synapses takes place in tens of milliseconds, while slow inhibition may last up to hundred milliseconds or longer [60, 130]. This difference in timescales allows a slow-fast analysis of the system. We measure the timescale separation by the ratio of the time constants of the excitatory and slow inhibitory synapses, i.e. we define $\epsilon$ as:

$$
\epsilon:=\frac{b}{a}
$$

To study the fast subsystem we consider the PSP induced by the slow inhibitory population, i.e. $x_{3}$, as constant. Essentially, this reduces the model to the Jansen and Rit neural mass [52]. We first study the fast dynamics of a single neural mass in absence of external input, i.e. we set $x_{5}=0$. The dynamics of the fast subsystem are described by Eqs. (5.2a), (5.2b) and (5.2d).

Using numerical continuation with MatCont [133], we have computed a bifurcation diagram for the fast subsystem varying $x_{3}$ as parameter. The result is shown in Figure 5.3. Note that, for visualization we show $u_{i s}$, rather than $u_{p y}$, as representative fast variable of the six-dimensional fast phase space. Starting at $x_{3}=0$, the system has a trivial equilibrium with $u_{i s}=0$, which is stable (solid line), also for higher $x_{3}$ values. Upon decreasing $x_{3}$, we find a fold bifurcation at $x_{3} \approx-0.023$ where the branch turns and the equilibrium loses stability (dashed blue line). The equilibrium regains stability at $x_{3} \approx 0.621$ via a second fold bifurcation. Next, there are two consecutive supercritical Hopf bifurcations at $x_{3} \approx 0.220$ and $x_{3} \approx 0.191$. For $x_{3}$ values between the Hopf points, the equilibrium is unstable and a stable limit cycle exists with a sinusoidal waveform and frequency of around $30 \mathrm{~Hz}$. Two further fold points for the equilibrium complete the diagram, but are not of interest for our study. Among the equilibrium curve of the fast subsystem there are three points for which the full system is at rest (coloured diamonds), i.e. $\left(x_{3}, u_{i f}\right)=(0,0)$ (stable, cyan), $(0.0862,0.558)$ (one unstable direction, gray) and $(0.618,2.55)$ (two unstable directions, orange). 


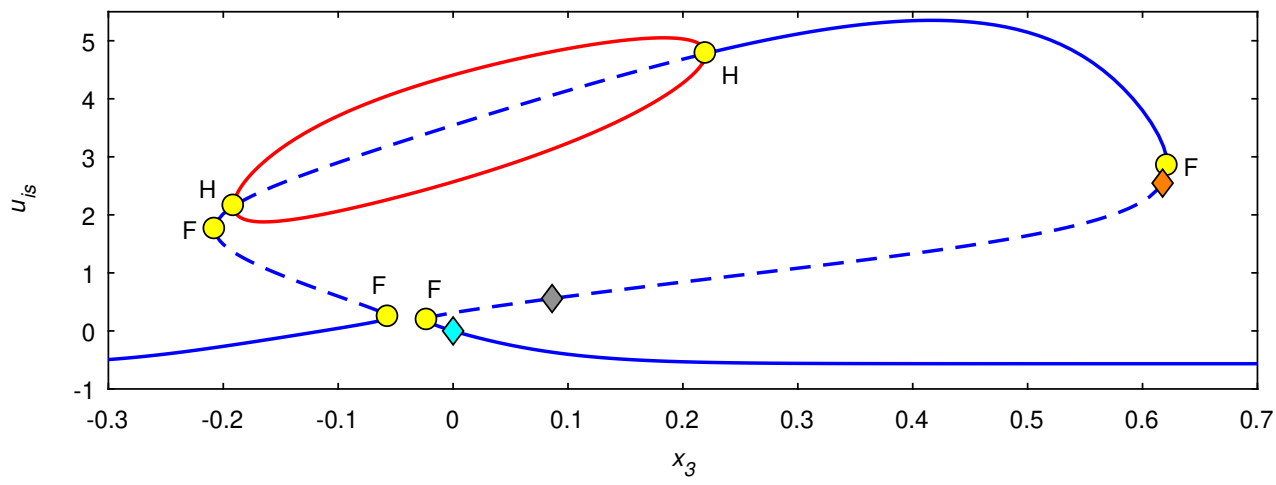

Figure 5.3: Bifurcation analysis of the fast subsystem varying $x_{3}$. Blue lines correspond to equilibria, while red lines indicate minimum and maximum values of $u_{i s}$ along the limit cycle. Solid lines represent stable solutions, dashed lines unstable ones. Yellow circles indicate fold $(\mathrm{F})$ and $\mathrm{Hopf}(\mathrm{H})$ bifurcations. Coloured diamonds indicate equilibria of the full system.

\subsubsection{Constant Input}

Before we consider time varying external input, i.e. the input arising from stimulation, we investigate the system for a fixed external input $x_{5}=-0.3$. For this external input the full system has a stable limit cycle and no stable equilibria. (We refer to Figure 4.13A for a bifurcation diagram of the full system.) Figure 5.4B shows that the shape of this limit cycle is similar to that of the simulated DRs.

Next, we study the limit cycle in the $x_{3}-u_{i s}$ diagram, combining it with the bifurcation diagram of the fast subsystem (see Figure 5.4A). This bifurcation diagram is quantitatively similar to the case without external input. The magenta line indicates the orbit of the LC. Starting from the lower branch of stable equilibria, $x_{3}$ decreases and the orbit follows this equilibrium branch until it reaches the fold bifurcation. Here the branch of stable equilibria vanishes and the fast subsystem jumps towards the stable limit cycle of the fast subsystem. Then $x_{3}$ increases and the orbit starts oscillating with a frequency corresponding to that of the limit cycle of the fast subsystem. As the multiplier of this limit cycle is close to one, the amplitude of the oscillations converges only slowly to the amplitude of the limit cycle. After passing the Hopf bifurcation, the oscillations damp out and the orbit approximately follows the upper branch of stable equilibria until this branch disappears in a fold bifurcation. Finally, the orbit jumps down to the lower branch of equilibria, and the cycle repeats.

The magenta line indicates the limit cycle for parameter values in Table 4.1. In this case the ratio between the timescales for excitation and slow inhibition $\epsilon=0.1$ is still relatively large for slow-fast analysis. We increase the separation of the timescales to $\epsilon=0.01$, by increasing the time constant of the slow inhibition to $b=1$. Meanwhile we keep $B / b$ constant as suggested by $[58,60]$, so the integral of the impulse response of the slow inhibitory synapses and the equilibria of the full system remain unchanged. Figure $5.4 \mathrm{C}$ shows the time profile of the limit cycle in this case, which has a much larger period now. When $\epsilon=0.01$, the orbit of the limit cycle follows the bifurcation diagram of the fast-system more closely (green line), compared to the orbit for $\epsilon=0.1$. 
A

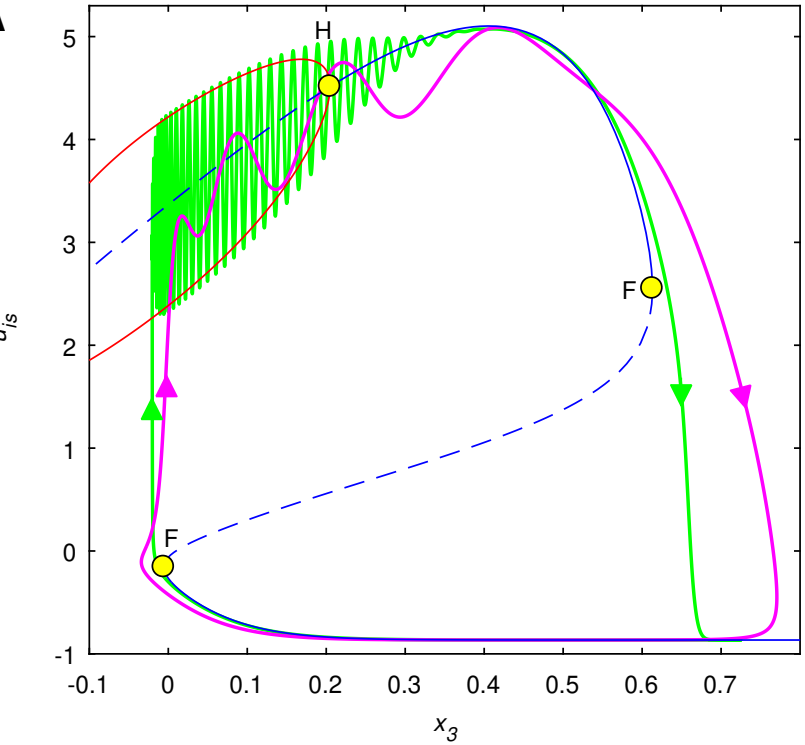

B
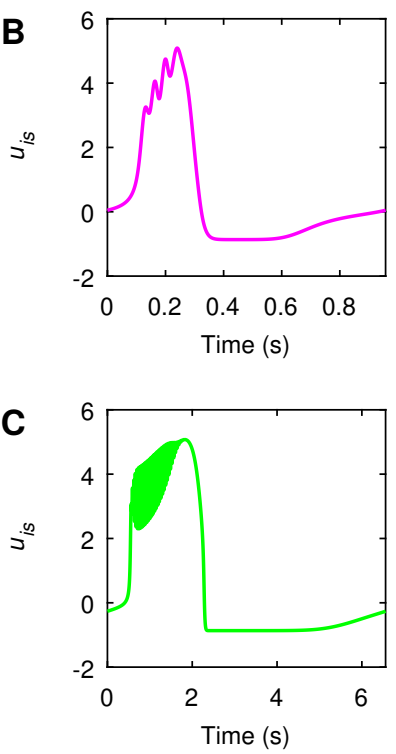

Figure 5.4: Slow-fast analysis of the limit cycle. (A) Bifurcation diagram of the fast subsystem varying $x_{3}$ for a constant external input $x_{5}=-0.3$. Blue and red lines denote equilibria and minimum $/$ maximum values of $u_{i s}$ along the limit cycle, respectively. Solid and dashed line styles indicate stable and unstable solutions and yellow circles represent fold $(\mathrm{F})$ and $\operatorname{Hopf}(\mathrm{H})$ bifurcations. The magenta and green lines indicate the orbits of the limit cycle of the full system for $\epsilon=0.1$ and $\epsilon=0.01$, respectively. Note that these orbits closely follow the equilibrium and limit cycle branches of the fast subsystem. (B, C) Time profiles of one period of the limit cycle for $\epsilon=0.1$ and $\epsilon=0.01$, respectively.

\subsubsection{Slowly Varying Input}

We now investigate the properties of the simulated secondary SPES responses shown in Section 5.2.2. Recall that the input from the first to the second neural mass varies slowly, in particular the return to baseline is slow corresponding to the second negative peak in the first neural mass. This motivates to use a slow-fast analysis to dissect the response of the second neural mass.

Considering both $x_{3}$ and $x_{5}$ as slowly varying parameters, we have computed the bifurcation diagram of the fast subsystem, see Figure 5.5A. With the additional slow parameter, there is a submanifold of equilibria. Projecting to the $\left(x_{3}, x_{5}, u_{i s}\right)$-space, we get a two-dimensional surface of equilibria (blue) with three bifurcation curves, i.e. two fold (light blue) and one supercritical Hopf (red). We also show the minimum/maximum of the stable limit cycle (red).

Figure 5.5A also contains the response to the stimulation for two $k$ values and $\epsilon=0.01$. For visualization purposes the initial fast transient of the responses is not shown. For $k=40$ (magenta line) the response stays on the lower part of the equilibrium manifold while it returns to the resting position marked with the cyan diamond, resulting in only a small response, see Figure 5.5B. For $k=80$ (green line) the input is sufficiently high to push the system beyond the fold bifurcation curve. This forces the fast subsystem to jump to the stable limit cycle leading to the rapid oscillations. While $x_{5}$ changes slowly, and accordingly $x_{3}$, the orbit 
A

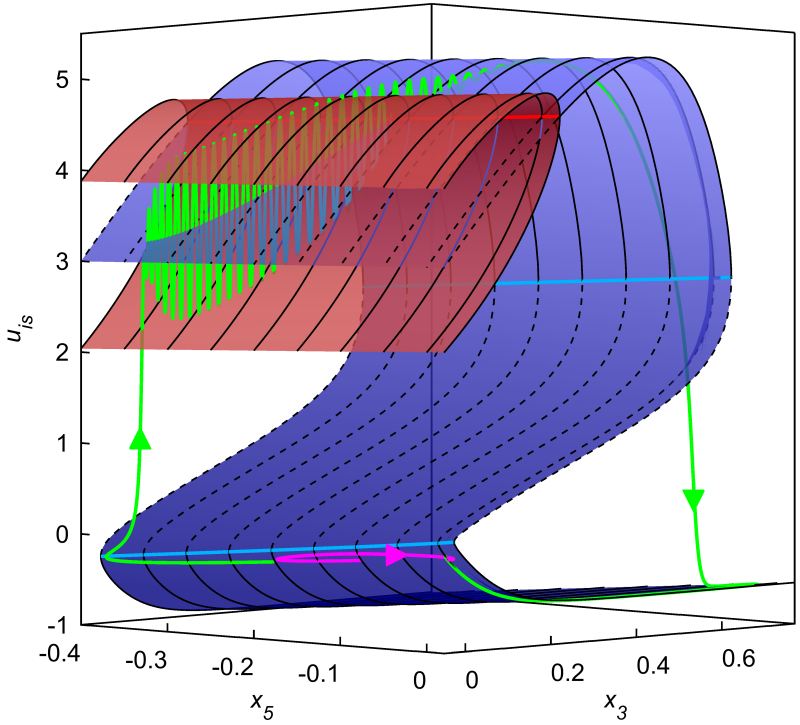

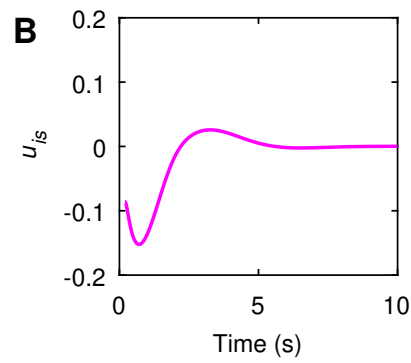

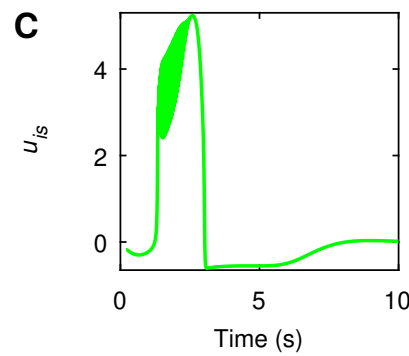

Figure 5.5: Slow-fast analysis of responses to slowly varing input. (A) Bifurcation diagram of the fast subsystem for $u_{i s}$ while varying $x_{5}$ and $x_{3}$. The blue folded surface represents equilibria, while the red surface indicates the minimum and maximum values of $u_{i s}$ along the stable limit cycle. The thin black lines indicate constant $x_{5}$ values on the surfaces and their line style, i.e. solid or dashed, indicates the stability of the solution. Stability of the equilibrium surfaces changes at the light blue and red lines representing fold and Hopf bifurcation curves, respectively. Simulated orbits are visualized by the magenta $(k=40)$ and green $(k=80)$ lines for $\epsilon=0.01$. (B, C) Time course of the simulated responses for $k=40$ and $k=80$, respectively.

passes the Hopf curve and the oscillations fade out. The orbit follows the top of the stable equilibrium surface until it reaches the second fold curve and jumps back to the bottom part of the equilibrium manifold. Finally, the system returns to the resting position on the lower equilibrium surface.

Next, we want to find $k^{*}$, the value of $k$ corresponding to the threshold between small and big responses. Figure 5.6 shows orbits for several values of $k$ near $k^{*}$. Observe that all orbits pass the fold bifurcation curve (light blue) and then remain for some time close to the middle part of the manifold corresponding to unstable equilibria, before leaving either upwards (green lines), resulting in a large amplitude response, or downwards (purple curves), giving a small response. The closer $k$ is to $k^{*}$, the longer the orbit stays near the unstable equilibrium surface. For $k^{*}$ the orbit will remain at the saddle equilibrium surface of the fast system, ending up in a saddle equilibrium of the full system. This saddle equilibrium satisfies $x_{5}=0$ as the external input returns to rest and is the equilibrium point marked with the gray diamond at $\left(x_{3}, u_{i s}\right) \approx(0,0862 ; 0.558)$ in the bifurcation diagram in Figure 5.3. This saddle equilibrium has one unstable direction, which is associated with the fast subsystem. So on the slow manifold this saddle equilibrium is attracting.

To determine $k^{*}$ and the associated orbit ending in the saddle equilibrium we formulate a boundary value problem (BVP) for the system of differential equations (5.2), with time 
A

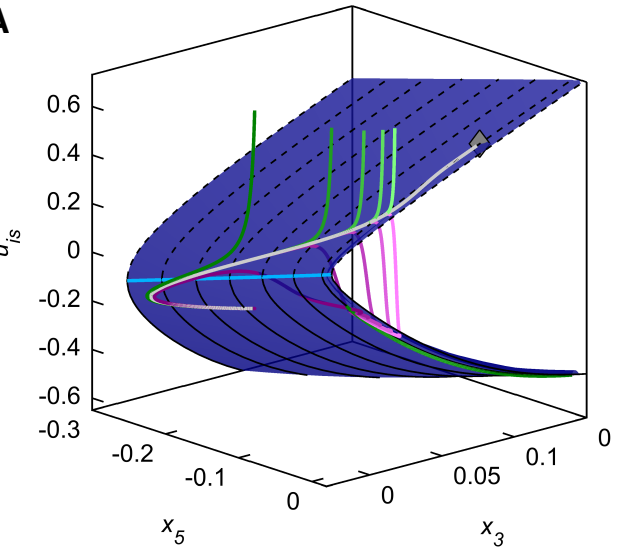

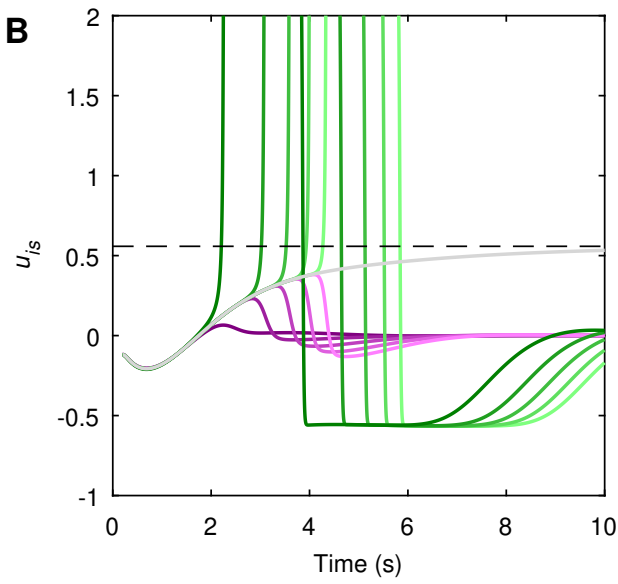

Figure 5.6: Simulated responses for $k$ values around the critical value $k^{*}$. (A) Responses plotted together with the fast bifurcation diagram (zoomed in on the bottom part). Green and purple lines denote simulated responses elapsing in big and small responses, respectively, where brighter colours correspond to $k$ values closer to $k^{*}$. The gray line indicates the orbit for $k^{*}$ found by solving the boundary value problem in Eq. (5.6), which ends up in the saddle equilibrium (gray diamond) and separates the two response types. (B) Simulated responses plotted against time. The dashed black line indicates the value of the saddle equilibrium of the full system.

varying input $x_{5}(t)=k \chi_{5}(t)$, where $k$ is the parameter and $\chi_{5}(t)$ is a (numerically) known function. We introduce $X=\left(x_{1}, \ldots, x_{4}, y_{1}, \ldots, y_{4}\right)$ and denote the saddle equilibrium as $X_{s}=\left(x_{s}, 0\right)$ and the orbit at $k^{*}$ as $X^{*}(t)$. The first boundary condition is that $X^{*}(t)$ starts at the stable equilibrium, i.e. $X^{*}(0)=0$. Next, we need $\lim _{t \rightarrow \infty} X^{*}(t)=X_{s}$. To have a finite orbit segment we truncate the orbit at time $T$. We assume that $T$ is sufficiently large such that the external input $x_{5}$ has vanished, and hence we can treat the system of differential equations as an autonomous system for $t>T$. Then, $X^{*}(T)$ should be in the stable manifold of $X_{S}$ in order for $X^{*}(t)$ to converge to this point [134]. Around the saddle equilibrium this manifold can be approximated by $L_{s}$, the right stable eigenspace of $X_{s}$. An equivalent condition for $X^{*}(T)$ in $L_{s}$ is that the projection of the difference $X^{*}(T)-X_{s}$ onto $L_{s}^{\perp}$ vanishes. Note that $L_{s}^{\perp}$ is identical to the left unstable eigenspace of $X_{s}$. As $X_{s}$ has one unstable direction, $L_{s}^{\perp}$ is spanned by the left eigenvector $q$ corresponding to the unstable direction of $X_{s}$. Hence, we require the inner-product between $q$ and $X^{*}(T)-X_{s}$ to be zero. This leads to the following nine boundary conditions:

$$
\begin{aligned}
X(0) & =0, \\
\left\langle X(T)-X_{s}, q\right\rangle & =0,
\end{aligned}
$$

for the eight dimensional system of differential equations (5.2) and free parameter $k$.

We take $T=15$ and solve the BVP numerically using the bvp4c function of Matlab with an absolute tolerance of $10^{-4}$, a relative tolerance of $10^{-2}$ and maximum of 160000 mesh points. This function requires an initial guess, which we obtain by simulating an orbit from $t=0$ to $t=2.5$ using a $k$-value close to the critical value such that the endpoint of the simulation is still close to the equilibrium manifold of the fast subsystem. We supplement 
the initial guess for the remaining time interval by using exponential decay towards $X_{S}$ at the rate given by the eigenvalue with negative real part and closest to the imaginary axis. The numerical solution of the BVP yields $k^{*} \approx 54.95$. The corresponding orbit is indicated by the gray line in Figure 5.6. We have $x_{5}(T) \approx-4.1 \cdot 10^{-7}$, indicating that $x_{5}$ indeed returned to rest. The computed orbit also gets close to the saddle equilibrium, i.e. the norm difference of the endpoint and the saddle is only 0.0021, another indication that the approximations of the BVP are valid.

The computed threshold $k^{*}$ separates the two response types. For $k<k^{*}$ the response falls back from the unstable manifold to the stable manifold at the bottom leading to a small response. For $k>k^{*}$ the system jumps from the unstable manifold to the stable manifold at the top, resulting in the large non-linear response.

\subsection{Conclusion \& Discussion}

We studied the transition between small, linear and large, non-linear responses in an excitable neural system with slowly varying input. The typical shape of the large non-linear responses, is explained by the interplay between a stable equilibrium and limit cycle in the bifurcation diagram of the fast subsystem. The critical response at the transition between the two response types can be characterized using a high-dimensional stable manifold. To determine the critical response and the parameter for which it arises, we formulated a boundary value problem. This boundary value problem can be used to study the parameter dependency of the transition, describing the boundary between normal and epileptic brain responses to stimulation.

\subsubsection{Limitations}

The bifurcation diagram of the fast subsystem explains the shape of the large responses qualitatively. The equilibria of the fast subsystem describe the manifold on which the slow dynamics acts if $\epsilon=0$, i.e. when the timescales are fully separated. This manifold perturbs to the slow manifold for $\epsilon>0$ as explained by Fenichel theory [151, 152] and extensions [153, 154]. The slow manifold will describe the orbit more accurately compared to the equilibria of the fast subsystem especially for bigger values of $\epsilon$. Computing these slow manifolds is a challenging task if they are of saddle type, like we have in our problem. In this case small errors in the unstable direction will yield rapid divergence from the manifold. Computing these manifolds requires advanced techniques [155].

To find the critical response ending in the saddle equilibrium of the full system we formulated a boundary value problem based on two assumptions. First, the external input must have vanished at the endpoint of the orbit segment. Second, the end point of the orbit must be sufficiently close to the saddle for the approximation by the hyper-plane to hold. Fulfilling both requirements results in a large time interval because of the systems' timescale separation, as well as many mesh points for an accurate approximation.

The external input we studied was given by a specific function, i.e. the reaction of another neural mass upon stimulation. Our analysis builds on two features of this function, namely its transient and slow nature. Therefore, one could expect similar results when replacing the input function by another function satisfying these two properties, also because the bifurcation diagram of the fast subsystem is almost invariant in the input parameter. We have experimented 
replacing the current $\chi_{5}(t)$ with a block pulse giving similar results.

\subsubsection{Slow Inhibition in the Wendling Model}

We exploit the timescale difference between slow inhibition one sides and fast inhibition and excitation other sides to study the Wendling model. Based on [130] we assume that slow inhibition models both slow $\mathrm{GABA}_{\mathrm{A}}$ and the even slower $\mathrm{GABA}_{\mathrm{B}}$ synaptic transmission. We have chosen the timescales within a physiologically plausible range such that the two characteristic peaks have a realistic timing [Chap. 4, 31, 130]. The ratio of the timescales yields $\epsilon=0.1$ justifying the slow-fast analysis, while in the original study the timescales were more similar, i.e. $\epsilon=0.5$ [53].

In our slow-fast analysis the fast subsystem reduces to the three population neural mass model proposed by [52]. Bifurcation analysis of this model has revealed the presence of three different attractors, i.e. stable equilibria, and limit cycles with a harmonic or a spiking shape $[131,156]$. Our analysis of the fast subsystem reveals only equilibria and harmonic oscillations, similar to [139] who found that the "spiking" limit cycle only exists if inhibition acts on a slower timescale than excitation. Under normal circumstances, these fast oscillations are suppressed by slow inhibition. External stimulation, however, may temporarily reduce this inhibitory restraint allowing the fast subsystem to move into a different dynamical regime. These fast oscillations also show up continuously when the synaptic gain of the slow inhibition, $B$, is close to zero. 

Chapter 6

General Discussion 
Patients with focal epilepsy might benefit from resective surgery. Successful epilepsy surgery requires an accurate delineation of the epileptogenic zone (EZ), i.e. the smallest area of cortex the removal of which removal yields seizure freedom [20,21], which is a challenging task. The aim of this thesis was to investigate the added value of combining computational network models and single pulse electical stimulation (SPES) for localizing the EZ. In this final chapter we will discuss how the results of the previous chapters can help to improve SPES. We discuss the challenges of applying computational network models for epilepsy surgery combined with SPES and finally we draw conclusions.

\subsection{Advancing Clinical SPES}

SPES can be used to probe both connectivity and epileptogenicity during iEEG recording. From a clinical perspective the occurrence of DRs is the most interesting as they are, in contrast to ERs, directly correlated with the EZ. The clinical usefulness of DRs for epilepsy surgery has been proven in multiple studies [28, 30, 39, 40]. An advantage of DRs over other biomarkers like spikes and HFOs is that DRs are evoked responses and not purely spontaneous events, which makes them more controlled. On the other hand, SPES is not applied in many centres for evoking DRs. Bottlenecks in the usage of SPES are the time needed to systematically stimulate over the whole grid and the labour intensive visual annotation of DRs afterwards.

\subsubsection{Accelerating the SPES Protocol}

To discover brain tissue exhibiting DRs, horizontally adjacent electrodes pairs over the whole grid are stimulated row by row. Due to the stochastic nature of DRs, multiple stimulation trials are needed to discover them. In the current SPES protocol at the UMC Utrecht electrode pairs are stimulated ten times at a frequency of $0.2 \mathrm{~Hz}$. For a typical patient with around 64 implanted electrodes this means that approximately one hour is needed to execute the whole protocol. The simplest way to speed-up the protocol would be reducing the inter stimulus time. However, too fast stimulation, e.g. at $1 \mathrm{~Hz}$, induces undesired short-term adaptation and plasticity [157].

A smart stimulation protocol would stimulate only a subset of all the electrode pairs instead of the whole grid. Such a protocol should omit stimulation pairs that can be expected to have a low chance of evoking DRs. On the other hand, based on the information of all stimulated electrode pairs one wants to find all spots capable of generating DRs, or at least those that are part of the EZ. To achieve this, a priori information might be incorporated, e.g. findings of preliminary investigations such as MRI scans.

Although it seems at first glance counterintuitive, information contained in the ER network may be used to accelerate the SPES protocol as well. ER networks can be constructed quickly due to the following two findings. First, ERs have, in contrast to DRs, a consistent appearance and hence, they can be reliably inferred using one to three stimulation trials instead of the usual ten. Second, ERs can be quickly identified from ECoG recordings using computationally efficient automatic detection algorithms, e.g. the one we have developed in chapter 3. So, ERs can be detected while recording ECoG data. Next, these ER networks can be used to determine relevant electrode pairs to stimulate with more trials in order to search for DRs. For example, one may stimulate electrode pairs that evoke many ERs more often as those pairs 
are more likely to evoke DRs [158]. Alternatively, as our results in chapter 4 show that DRs might be seen as second-order responses in the ER network, one may want to stimulate more often electrode pairs from which many electrodes can be reached with length two paths. In this way many locations at once are probed for DRs. A more advanced combination of data analysis, graph analysis and modelling of SPES responses might be considered to improve these rules. Finally, we note that information about epileptogenicity might, to some extent, be inferred from properties of ERs such as amplitude [159] and frequency content [36, 160] or from network measures applied to ER networks [37, 38].

\subsubsection{Examination of DRs}

The detection of DRs is up till now another time consuming aspect of SPES. Currently, visually annotating the response in the time-frequency domain is the gold standard to analyze DRs responses in the UMC Utrecht. For every stimulation pair a time-frequency image is scored in three different frequency bands for each electrode by two independent observers. For a typical patient with 64 implanted electrodes this means that around 3600 images must be scored. The number of time-frequency images grows quadratically with the number of implanted electrodes, as increasing the number of electrodes also increases the number of stimulation pairs. So, for patients with more implanted electrodes the number of images to analyze grows quickly.

An alternative, less time consuming method to examine DRs is by creating one timefrequency image per electrode instead of one image for each combination of stimulation pair and response electrode. This single image can be obtained by averaging the responses of that electrode over all stimulation trials regardless of the stimulation pair. Such an image then shows the mean response of an electrode. For a typical patient this method yields around 64 images, which can be used to obtain a quick overview of the interesting electrodes. For these electrodes the time-frequency images per stimulation pair may than be assessed. A disadvantage of averaging over all stimulation trials is that the power of DRs in the timefrequency image is diminished as also stimulation pairs that do not evoke responses are taken into account. One might overcome this by averaging only over the trials belonging to a subset of the stimulation pairs from which one could expect a priori that they contain relatively many DRs, e.g. those pairs that evoke the most ERs [161]. Actually, this method requires the same techniques as described above to shorten the SPES protocol and hence it can be perfectly combined with a more efficient SPES protocol.

Automatic detection of DRs offers another possibility to drastically reduce the analysis time. A further advantage is that automatic detection yields a consistent classification, while visual analysis by human observers will always remain subjective. Automatic detection of DRs, however, is a non trivial task due to the stochastic occurrence of DRs and their varying latency and waveform. Traditional methods like thresholding and template matching are not sufficiently definite $[162,163]$, so more advanced machine learning techniques are required. Machine learning for DRs can be applied in the time-frequency or time domain. Using the same time-frequency images as used for visual examination, two different machine learning methods showed promising results, especially for spikes and riples [164]. Compared to the inter-observer agreement between two humans, the agreement between human and machine was similar for both methods in the spike and ripple bands [165]. Detection of DRs by applying machine learning to the time series is an interesting next step. As it skips the 
generation of the time-frequency images, automatic detection in the time domain is likely to be faster than detection in the time-frequency domain.

Machine learning offers possibilities to further discriminate types of DRs using features that have not been recognized by humans. For example, during the learning process one can incorporate whether or not a response was recorded at an electrode part of the resected area (RA) to learn the specific properties of the responses evoked at those electrodes [165]. Using such an approach, DRs might be divided in classes of which some might be more specific for the EZ than others. There might, for instance, exist physiological and pathological DRs, just as there exist physiological and pathological HFOs [27, 166].

Finally, the occurrence of DRs has so far only been studied at an electrode level, i.e. for each electrode is determined how often a DR is evoked. Our results in chapter 4 suggest that network effects also play an important role in the generation of DRs. Also, when emerged, the activity of DRs might spread through the network. These notions are enhanced by the impression we got while visually marking the onset of DRs in the SPES data of chapter 4, namely that a single stimulation trial either evoked DRs on multiple electrodes or no DRs at all. It is, therefore, interesting to study the occurrence of DRs not only as electrode-wise events, but also as a spatial-temporal phenomenon.

\subsubsection{Intra-Operative Use of Spes}

Up till now, the use of SPES to assist with localizing the EZ is only applied during long-term iEEG monitoring. Such a monitoring period usually takes a couple of days. Therefore, the time to execute the SPES protocol and to analyse the results is not really a constraint. On the other hand, this limits the use of SPES to the small group of patients for which long-term ECoG monitoring is required.

Epilepsy surgery is applied to a much larger group of patients in some of which ECoG recordings are performed intra-operatively to taylor the resection [167]. As intra-operative ECoG is recorded for a short time, typically 15-30 minutes, only interictal events, traditionally being spikes and recently also HFOs, are considered as biomarkers for the EZ [168, 169]. In fact, as these biomarkers are spontaneous events, their occurrence is uncontrolled and therefore they might be missed during short recordings. SPES, in contrast, would offer a controlled way to probe epileptogenicity, as it is evoking responses.

An important issue that must be addressed before SPES could be applied intra-operatively for localizing the EZ, is the influence of sedation on the evoked responses, especially DRs. Studies where SPES has been applied intra-operatively to map the dorsal language network report that amplitude of ERs is reduced under general anesthesia, but still could be well recorded [170,171]. Mathematical modelling also allows to study the effects of sedation on neuronal activity [172]. Hence, by integrating these mechanisms into the model for SPES responses in chapter 4, the influence of sedation on the occurrence of DRs can be studied. Combined with the ideas to reduce execution and analysis time this might make the intraoperative use of SPES for precise localization of the EZ feasible, although still many efforts need to be made. 


\subsection{Network Models for Epilepsy Surgery}

There is increasing evidence that epilepsy is to some extent a network disease [70, 83, 173]. The presurgical evaluation of epilepsy patients may, therefore, benefit from the study of brain networks [85]. Network measures can be used to characterize the network structure. Such measures describe properties of individual nodes, e.g. degree and centrality, or the global network, e.g. clustering coefficient and average path length between nodes [88]. Using global network measures, multiple studies have shown that brain networks of epilepsy patients have a different network topology compared to healthy controls [71-73]. Also, global network measures indicate that functional connectivity in epilepsy patients changes before and during seizures [174-177]. Network measures characterizing individual nodes try to describe the importance of a node to the whole network. In different types of networks it has been found that nodes in the SOZ/RA are often more influential compared to non-SOZ/RA nodes in the sense that on average the degree, net outflow, and centrality of SOZ/RA nodes is elevated [38, 105, 107, 178-181]. However, it has not been investigated how network analysis can be used to plan the extent of the resection, which might be one of the reasons that it is not used in standard clinical practice [84].

A promising, recent development is to enrich networks with dynamics $[65-67,76]$. In such computational network models, each node is a neural mass or a similar phenomenological model capable of generating interictal and epileptiform activity. The connections indicate how the different nodes can influence each others' dynamics, while the internal parameters of a node govern its excitability. These models allow to study the joint influence of two factors playing a role in epilepsy, i.e. altered connectivity and enhanced local excitability due to local tissue deviations. Another attractive aspect of computational network modes is that they offer a framework to experiment with strategies for epilepsy surgery [Chap. 2, 65, 66]. In these models surgery can be modelled by removing nodes from the network. Next, the effect can be evaluated by comparing simulations of the reduced network with baseline simulations of the full network.

In chapter 2 we considered a computational network model consisting of 4 nodes. This model demonstrates that besides local excitability it is important to take into account the network structure both before and after surgery. Other studies have considered larger, patientspecific models $[65,66,101,182]$ to predict the effect of surgery.

In [101] a bistable oscillator, slightly different from the one in chapter 2, was used in combination with correlation networks derived from interictal ECoG. Using model simulations the escape time, i.e. the time it took for a node to go from the interictal to ictal state, was determined for each node. Next, the in silico SOZ was determined by the nodes that had the shortest escape time. In five of the six patients this in silico SOZ overlapped with the clinically defined SOZ.

In a subsequent study by the same group [66] the same model was used except that, for some unreported reason, the coupling between the nodes in the model was slightly changed. It was found that in some, but not all, of the patients the in silico SOZ agreed with the clinical SOZ. Moreover, by comparing the effect of the removal of the clinically resected area to at random resection in the model it was possible to predict the surgical outcome in 13 out of 16 patients correctly.

Another study [65] used the Wendling neural mass model with seizure networks derived using mutual information. To quantify the epileptogenicity of a network, the term brain net- 
work ictogenicity was introduced as the fraction of the time the model exhibited epileptiform discharges. Next, the ictogenicity of a node was defined as the relative improvement in the brain network ictogenicity upon removal of that node. In patients with a good surgical outcome the maximum node ictogenicity among the clinically resected nodes was significantly higher than in patients where the surgical outcome was poor. Also, removal of the clinically resected area in the model yielded a significantly higher improvement in brain network ictogenicity in patients with a good surgical outcome compared to those with a poor outcome.

In a subsequent study [182] it was shown that node ictogenicity correlates with network measures like degree and eigenvalue centrality for different network topologies, i.e. random, small-world, scale-free and rich-club networks. Using the same patient population as in [65], but correlation instead of mutual information networks, it was found that patient networks exhibited a rich-club structure. From simulation results it was expected that in those networks removal of the rich-club, i.e. a certain set of nodes with a high-degree, would be effective in reducing the seizure rate. This hypothesis was confirmed as patients with a good surgical outcome had a significantly larger part of the rich-club nodes resected than those with a poor outcome.

Despite all the promising results in these studies there are still many challenges regarding both the inference of patient-specific connectivity and the incorporation of local tissue properties that must be tackled before computational network models can be used in clinical practice prospectively.

\subsubsection{Inferring Connectivity}

Patient-specific connectivity for computational network models can be obtained from different sources, e.g. iEEG recordings or diffusion weighted imaging (DWI), leading to networks at centimeter-scale or whole brain level, respectively. Until now, most studies have used functional connectivity networks derived from iEEG recordings [65, 66, 101, 119, 182]. These networks are inferred from ongoing brain activity either during seizures or interictal periods. Deducing connectivity in this way is a non trivial problem, as simultaneously one tries to reconstruct the presence of $N(N-1)$ possible connections by observing $N$ electrodes. Numerous methods, all with their own pros and cons, have been developed. These methods are based on different mathematical principles and make different assumptions on the processes underlying the data [89]. Besides this, also the pre-processing of the iEEG data, e.g. the choice of the reference montage, the frequency band and the length of the epochs, will influence the reconstructed network $[115,183,184]$.

SPES offers an alternative to probe connectivity in an active way. The stimulus-response relation yields directional connectivity in a more natural way compared to functional connectivity methods and requires fewer methodological choices to be made. In chapter 3 we compared two traditional methods for functional connectivity, i.e. cross-correlation (CC) and Granger causality (GC), with SPES ER networks. Functional connectivity networks revealed to some extent a similar network as SPES, especially the cross-correlation networks formed almost a subnetwork of the SPES network. However, SPES was capable to infer more distant connections as well. These distant connections might not be observed when connectivity is inferred from ongoing iEEG recordings as the influence of these connections might be overpowered by densely connected local clusters. For this reason physiological long-range connections may be missed by functional connectivity methods. For example in chapter 3 
we found that $\mathrm{CC}$ and $\mathrm{GC}$ networks are not able to capture connections between Broca and Wernicke, while multiple studies have reported that these connections can be found using SPES [34, 113, 170, 171].

A possible disadvantage of networks derived from SPES is that these networks only capture interictal connectivity. Functional connectivity changes during seizures $[176,177$, 182]. One may ask to what extent the observed changes in connectivity resemble changes of the underlying network or whether these observed changes are only a consequence of the change in dynamics. In the latter case, the observed network changes due to limitations of the functional connectivity measure, e.g. as the stationarity or linearity assumptions on the underlying data are violated. On the other hand, the question is to what extent the observed functional networks can be used to predict the EZ. A reasonable hypothesis is that in ictal networks pathological network structures are more pronounced compared to interictal ones and that therefore ictal networks are more informative. From a clinical point of view the use of interictal networks would be preferable as this does not require the need to record a seizure. So far it seems that both functional networks derived from seizures $[65,119,182]$ and interictal recordings $[66,101]$ contain sufficient information to predict the clinical outcome of the resection using computational network models. For SPES networks this needs to be investigated. It is at least a good sign that SPES networks can (partially) explain seizure propagation $[35,36]$.

A limitation of networks derived from iEEG recordings is that they only capture a part of the brain network. This issue is especially relevant when grid electrodes are used, as they sample only activity at the surface of a limited area. It is good to realize that the actual EZ might be outside the area covered by the grid. Also, one must be aware of boundary effects which may influence the result of the computational network model. For example, in the model we used in chapter 2 nodes receiving less input were more vulnerable to escape to the ictal state. As most connections are local [Chap. 3], nodes at the boundary of a grid are typically less connected in the observed network and hence they might be wrongly identified as epileptogenic nodes. This boundary effect might be overcome by introducing extra nodes connected to the boundary which model the 'surrounding brain' and have a low intrinsic epileptogenicity. One might think for example of incorporating a patient-specific network into a less detailed full-brain network. For SPES networks a good option might be to fit the detailed patient-specific network into one of the generic brain atlases available for SPES [98, 116].

An alternative solution to the boundary effect is to infer connectivity using DWI. The resulting networks resemble structural connectivity on a whole brain scale and hence boundary effects are eliminated. A computational modelling study using DWI networks showed a significant differences in the probability to generate seizures between patients with temporal lobe epilepsy and healthy controls on a group level [185]. Also, for individual patients it is possible to predict seizure propagation using computational network models[67, 186]. These results are encouraging for the use of connectivity derived from DWI in computational network models to assist in determination of the optimal targets for epilepsy surgery, although the resolution of DWI networks might be too crude to taylor resection precisely. To increase precision, DWI networks may be combined with more detailed local SPES networks as to some extent these two networks show similar connectivity between certain clusters of brain regions $[97,98]$. 


\subsubsection{Measuring Intrinsic Excitability}

Computational network models have the advantage that not only network structure but also local excitability is taken into account. In this way these models add to network studies that consider only graph metrics to identify epileptogenic network structures, while neglecting local tissue properties. However, the current computational modelling studies aiming at predicting the effect of epilepsy surgery have not discriminated the parameters governing the intrinsic dynamics of the nodes. These models are only considering network effects and, as a result, the epileptogenicity of nodes can also be inferred using network measures [87, 119]. In clinical practice, however, lesions and events in (intracranial) EEG recordings such as interictal spikes, HFOs and DRs to SPES have proven to be good biomarkers for epileptogenicity $[21,22,26,39]$ and are heavily used in presurgical evaluation. As a next step to improve computational network models it seems logical to include this information.

DRs evoked during SPES may offer a good way to examine local excitability. In chapters 4 and 5 we provided a mechanistic explanation for the occurrence of DRs. First of all, data analysis showed that DRs can be seen as indirect responses rather than direct responses to the stimulation, which explains their relatively late occurrence compared to ERs. Propagating activity of ERs, or, more precisely, a temporary reduction in slow inhibition due to ERs, may than elicit DRs at a neuronal population. This mechanism implies that besides the local excitability of a neuronal population also its location in the network is of importance for the generation of DRs. More precisely, our model simulations, using two feed-forward coupled neural masses, show a reciprocal relation between the strength of the ingoing connections to a neural mass and its intrinsic excitability regarding the ability of the neural mass to exhibit DRs. So, to use DRs as a measure for excitability one should compensate for the location of the node in the network.

An advantage of taking DRs evoked during SPES as measure for excitability is that the occurrence of DRs is controlled. Interictal spikes and HFOs, on the contrary, are spontaneous events occurring during interical iEEG. The question is to what extent DRs offer a substitute to spikes and HFOs. In some sense one may argue that DRs simply represent evoked spikes and HFOs.

It seems plausible that DRs with a spike component are similar to interictal spikes. At least the DRs simulated using the Wendling neural mass model in chapter 4 share the same underlying mechanism in the model as interictal spikes, i.e. both are the result of a temporary excursion to a limit cycle resembling epileptiform activity. This notion is further supported by the similarity in morphology of the waveforms of spikes and DRs observed in both ECoG [142] and single neuron recordings [141].

Whether ripple and fast-ripple activity of DRs share a similar mechanism as spontaneous HFOs, is less clear. The neural mass model we studied in chapters 4 and 5 was not capable to generate such high frequency activity $(>80 \mathrm{~Hz})$ and so there is currently no evidence from a modelling point of view. In ECoG recordings it was found that both evoked and spontaneous ripples and fast-ripples occur in a similar proportion inside and outside the SOZ [187]. All together it seems reasonable that DRs offer a reasonable alternative for spontaneous interictal spikes and HFOs with the advantage that they are more controlled. 


\subsubsection{Recommendations}

Multiple studies have considered the use of computational network models for epilepsy surgery, each of them using a slightly different methodology [65-67, 101, 182, 186]. These differences concern the choice of the network (ictal or interictal and constructed method, e.g. correlation or mutual information) and the model used for the node dynamics (neural mass model, the Epileptor, bistable oscillators). This raises the question which methodology yields the best result. Recently, a comparison of models for the node dynamics showed that in sparse (artificial) networks the ranking of nodes according to their node ictogenicity was highly similar for three different models, while in dense networks the rankings differed [188]. To test the influence of other methodological aspects, a side-by-side comparison of the different methodologies is useful next step. The best way to perform such a comparison is by using a new independent dataset. Such a study would then also be a verification of the previous studies and, if successful, this will increase the credibility of computational network models.

To advance the use of computational network models for epilepsy surgery even further it is also important to incorporate node dependent information about local excitability. In this way the dynamics on the network is of added value and ensures that computational network models go beyond the limitation of pure network analysis methods. Delayed responses evoked during SPES might be a good candidate to gauge the excitability of nodes. The value of this biomarker was already proven in clinical practice and the results in chapter 4 provide a theoretical underpinning of this. Also, from a practical point of view the use of DRs is beneficial as there occurrence can be probed in a controlled fashion.

\subsection{Conclusion}

Computational network models offer a useful framework to assist with delineation of the epileptogenic zone. These models provide a method to investigate both the role of network structure and local tissue properties in seizure generation and to test the effect of surgery. Taking into account network structure during surgery is important, as removal of normal tissue located at a crucial spot in the network might in some cases be more effective than removing hyperexcitable tissue. The patient-specific information required for computational network models might be obtained using SPES. SPES offers a straightforward method to probe directional connectivity. Compared to some functional connectivity methods, SPES ER networks have the advantage of incorporating more physiological connections. Moreover, the occurrence of delayed responses evoked with SPES gives information about the local excitability of brain tissue, provided that network effects are taken into account. In conclusion, SPES and patient-specific computational network models form a promising combination that have great potential to improve epilepsy surgery. 



\section{Appendices}

List of Abbreviations

Bibliography

Summary

Samenvatting

Dankwoord 


\section{List of Abbreviations}

CC - cross-correlation

CCEP - cortico-cortical evoked responses

DR - delayed response

ECoG - electrocorticography

EEG - electroencephalography

ER - early response

ESM - electrocortical stimulation mapping

EZ - epileptogenic zone

GC - Granger causality

HFA - high-frequency activity

HFO - high-frequency oscillation

iEEG - intracranial EEG

MEG - magnetoencephalography

MRI - magnetic resonance imaging

NMM - neural mass model

RA - resected area

SEEG - stereotactic EEG

SOZ - seizure onset zone

SPES - single pulse electrical stimulation 


\section{Bibliography}

[1] Sander JW. The epidemiology of epilepsy revisited. Current opinion in neurology 16.2 (2003), 165-170. DOI: 10.1097/01.wCo.0000063766.15877.8e.

[2] Bell GS, Neligan A, and Sander JW. An unknown quantity-the worldwide prevalence of epilepsy. Epilepsia 55.7 (2014), 958-962. Dor: 10.1111/epi . 12605.

[3] Fisher RS, Van Emde Boas W, Blume W, Elger C, Genton P, Lee P, and Engel Jr J. Epileptic seizures and epilepsy: definitions proposed by the International League Against Epilepsy (ILAE) and the International Bureau for Epilepsy (IBE). Epilepsia 46.4 (2005), 470-472. DOI: 10.1111/j .0013-9580.2005.66104.x.

[4] Fisher RS, Acevedo C, Arzimanoglou A, Bogacz A, Cross JH, Elger CE, Engel Jr J, Forsgren L, French JA, Glynn M, Hesdorffer DC, Lee B, Mathern GW, Moshé SL, Perucca E, Scheffer IE, Tomson T, Watanabe M, and Wiebe S. ILAE official report: a practical clinical definition of epilepsy. Epilepsia 55.4 (2014), 475-482. DoI: $10.1111 /$ epi. 12550.

[5] Scheffer IE, Berkovic S, Capovilla G, Connolly MB, French J, Guilhoto L, Hirsch E, Jain S, Mathern GW, Moshé SL, Nordli DR, Perucca E, Tomson T, Wiebe S, Zhang Y, and Zuberi SM. ILAE classification of the epilepsies: position paper of the ILAE Commission for Classification and Terminology. Epilepsia 58.4 (2017), 512-521. DOI: 10.1111 /epi. 13709 .

[6] Fisher RS, Cross JH, French JA, Higurashi N, Hirsch E, Jansen FE, Lagae L, Moshé SL, Peltola J, Roulet Perez E, Scheffer IE, and Zuberi SM. Operational classification of seizure types by the International League Against Epilepsy: Position Paper of the ILAE Commission for Classification and Terminology. Epilepsia 58.4 (2017), 522 530. DoI: 10.1111 /epi . 13670.

[7] Berg AT, Berkovic SF, Brodie MJ, Buchhalter J, Cross JH, Van Emde Boas W, Engel J, French J, Glauser TA, Mathern GW, Moshé SL, Nordli D, Plouin P, and Scheffer IE. Revised terminology and concepts for organization of seizures and epilepsies: report of the ILAE Commission on Classification and Terminology, 2005-2009. Epilepsia 51.4 (2010), 676-685. DoI: $10.1111 / \mathrm{j} .1528-1167.2010 .02522$. x.

[8] Schuele SU and Lüders HO. Intractable epilepsy: management and therapeutic alternatives. The Lancet Neurology 7.6 (2008), 514-524. DoI: 10 . 1016 / S14744422 (08) 70108-X.

[9] Duncan JS, Sander JW, Sisodiya SM, and Walker MC. Adult epilepsy. The Lancet 367.9516 (2006), 1087-1100. Dor: 10.1016/S0140-6736(06)68477-8.

[10] Löscher W and Schmidt D. Modern antiepileptic drug development has failed to deliver: ways out of the current dilemma. Epilepsia 52.4 (2011), 657-678. Dor: 10 . 1111/j.1528-1167.2011.03024.x.

[11] Sander JW. The use of antiepileptic drugs—principles and practice. Epilepsia 45 (2004), 28-34. DOI: 10.1111/j.0013-9580.2004.455005.x.

[12] Brodie MJ. Antiepileptic drug therapy the story so far. Seizure 19.10 (2010), 650-655. DoI: $10.1016 / \mathrm{j}$. seizure. 2010.10 .027 . 
[13] Kwan P, Arzimanoglou A, Berg AT, Brodie MJ, Allen Hauser W, Mathern G, Moshé SL, Perucca E, Wiebe S, and French J. Definition of drug resistant epilepsy: consensus proposal by the ad hoc Task Force of the ILAE Commission on Therapeutic Strategies. Epilepsia 51.6 (2010), 1069-1077. Dor: 10.1111/j . 1528-1167.2009.02397.x.

[14] Schmidt D. Efficacy of New Antiepileptic Drugs: Efficacy of New Antiepileptic Drugs. Epilepsy Currents 11.1 (2011), 9-11. Dor: 10.5698/1535-7511-11.1.9.

[15] Vassileva A, Van Blooijs D, Leijten F, and Huiskamp G. Neocortical electrical stimulation for epilepsy: Closed-loop versus open-loop. Epilepsy research 141 (2018), 95-101. Dor: 10.1016/j .eplepsyres .2018.02.010.

[16] Boon P, Raedt R, De Herdt V, Wyckhuys T, and Vonck K. Electrical stimulation for the treatment of epilepsy. Neurotherapeutics 6.2 (2009), 218-227. DoI: 10.1016/j . nurt.2008.12.003.

[17] Fisher RS and Velasco AL. Electrical brain stimulation for epilepsy. Nature Reviews Neurology 10.5 (2014), 261. Dor: 10.1038/nrneurol . 2014.59.

[18] Jobst BC and Cascino GD. Resective epilepsy surgery for drug-resistant focal epilepsy: A review. Journal of the American Medical Association 313.3 (2015), 285-293. DoI: 10.1001/jama.2014.17426.

[19] Malmgren K and Edelvik A. Long-term outcomes of surgical treatment for epilepsy in adults with regard to seizures, antiepileptic drug treatment and employment. Seizure 44 (2017), 217-224. DoI: $10.1016 / \mathrm{j}$. seizure .2016.10.015.

[20] Rosenow F and Lüders H. Presurgical evaluation of epilepsy. Brain 124.9 (2001), 1683-1700. DoI: 10.1093/brain/124.9.1683.

[21] Lüders HO, Najm I, Nair D, Widdess-Walsh P, and Bingman W. The epileptogenic zone: General principles. Epileptic Disorders 8.supplement 2 (2006), S1-S9.

[22] Téllez-Zenteno JF, Ronquillo LH, Moien-Afshari ., and Wiebe S. Surgical outcomes in lesional and non-lesional epilepsy: a systematic review and meta-analysis. Epilepsy research 89.2-3 (2010), 310-318. Dor: 10.1016/j . eplepsyres .2010.02 .007.

[23] Lopes da Silva F. EEG and MEG: relevance to neuroscience. Neuron 80.5 (2013), 1112-1128. DoI: $10.1016 / \mathrm{j}$. neuron . 2013.10 .017$.

[24] Nair DR, Burgess R, McIntyre CC, and Lueders H. Chronic subdural electrodes in the management of epilepsy. Clinical neurophysiology 119.1 (2008), 11-28. DoI: 10.1016/j.clinph.2007.09.117.

[25] Lesser RP, Crone NE, and Webber WRS. Subdural electrodes. Clinical Neurophysiology 121.9 (2010), 1376-1392. Dor: 10.1016/j . clinph. 2010.04 .037$.

[26] Jacobs J, Zijlmans M, Zelmann R, Chatillon C, Hall J, Olivier A, Dubeau F, and Gotman J. High-frequency electroencephalographic oscillations correlate with outcome of epilepsy surgery. Annals of Neurology: Official Journal of the American Neurological Association and the Child Neurology Society 67.2 (2010), 209-220. DOI: 10.1002 /ana. 21847. 
[27] Jacobs J, Staba R, Asano E, Otsubo H, Wu J, Zijlmans M, Mohamed I, Kahane P, Dubeau F, Navarro V, and Gotman J. High-frequency oscillations (HFOs) in clinical epilepsy. Progress in neurobiology 98.3 (2012), 302-315. DoI: 10.1016/j . pneurobio.2012.03.001.

[28] Valentín A, Anderson M, Alarcón G, García Seoane JJ, Selway R, Binnie CD, and Polkey CE. Responses to single pulse electrical stimulation identify epileptogenesis in the human brain in vivo. Brain 125.8 (2002), 1709-1718. Dor: 10.1093/brain/ awf187.

[29] Valentín A, Alarcón G, García-Seoane JJ, Lacruz ME, Nayak SD, Honavar M, Selway RP, Binnie CD, and Polkey CE. Single-pulse electrical stimulation identifies epileptogenic frontal cortex in the human brain. Neurology 65.3 (2005), 426-435. DOI: $10.1212 / 01 . w n l .0000171340 .73078 . c 1$.

[30] Flanagan D, Valentín A, García Seoane JJ, Alarcón G, and Boyd SG. Single-pulse electrical stimulation helps to identify epileptogenic cortex in children. Epilepsia 50.7 (July 2009), 1793-1803. Dor: 10.1111/j .1528-1167.2009.02056.x.

[31] Alarcón G, Jiménez-Jiménez D, Valentín A, and Martín-López D. Characterizing EEG Cortical Dynamics and Connectivity with Responses to Single Pulse Electrical Stimulation (SPES). International Journal of Neural Systems 28.6 (2018), 1750057. DOI: $10.1142 /$ SQ129065717500575.

[32] Lacruz ME, García Seoane JJ, Valentín A, Selway R, and Alarcón G. Frontal and temporal functional connections of the living human brain. European Journal of Neuroscience 26.5 (2007), 1357-1370. DoI: 10.1111/j . 1460-9568 . 2007 . 05730. $\mathrm{x}$.

[33] Keller CJ, Honey CJ, Mégevand P, Entz L, Ulbert I, and Mehta AD. Mapping human brain networks with cortico-cortical evoked potentials. Philosophical Transactions of the Royal Society B: Biological Sciences 369.1653 (2014). DoI: 10 . 1098 /rstb. 2013.0528.

[34] Matsumoto R, Kunieda T, and Nair D. Single pulse electrical stimulation to probe functional and pathological connectivity in epilepsy. Seizure 44 (2017), 27-36. Dor: 10.1016/j.seizure.2016.11.003.

[35] Enatsu R, Jin K, Elwan S, Kubota Y, Piao Z, O'Connor T, Horning K, Burgess RC, Bingaman W, and Nair DR. Correlations between ictal propagation and response to electrical cortical stimulation: A cortico-cortical evoked potential study. Epilepsy research 101.1-2 (2012), 76-87. Dor: 10.1016/j .eplepsyres .2012.03.004.

[36] Mouthaan BE, Van 't Klooster MA, Keizer D, Hebbink GJ, Leijten FSS, Ferrier CH, Van Putten MJAM, Zijlmans M, and Huiskamp GJM. Single Pulse Electrical Stimulation to identify epileptogenic cortex: Clinical information obtained from early evoked responses. Clinical Neurophysiology 127.2 (2016), 1088-1098. DOI: 10 . 1016/j .clinph.2015.07.031.

[37] Boido D, Kapetis D, Gnatkovsky V, Pastori C, Galbardi B, Sartori I, Tassi L, Cardinale F, Francione S, and De Curtis M. Stimulus-evoked potentials contribute to map the epileptogenic zone during stereo-EEG presurgical monitoring. Human brain mapping 35.9 (2014), 4267-4281. DOI: 10. 1002/hbm. 22516. 
[38] Van Blooijs D, Leijten FSS, Van Rijen PC, Meijer HGE, and Huiskamp GJM. Evoked directional network characteristics of epileptogenic tissue derived from single pulse electrical stimulation. Human Brain Mapping (2018). DoI: 10. 1002/hbm . 24309.

[39] Valentín A, Alarcón G, Honavar M, García Seoane JJ, Selway RP, Polkey CE, and Binnie CD. Single pulse electrical stimulation for identification of structural abnormalities and prediction of seizure outcome after epilepsy surgery: a prospective study. The Lancet Neurology 4.11 (2005), 718-726. DoI: 10 . 1016/S1474-4422 (05) 70200-3.

[40] Van 't Klooster MA, Zijlmans M, Leijten FSS, Ferrier CH, Van Putten MJAM, and Huiskamp GJM. Time-frequency analysis of single pulse electrical stimulation to assist delineation of epileptogenic cortex. Brain 134.10 (2011), 2855-2866. Dor: 10. 1093/brain/awr211.

[41] Wendling F. Computational models of epileptic activity: a bridge between observation and pathophysiological interpretation. Expert review of neurotherapeutics 8.6 (2008), 889-896. DoI: 10.1586/14737175.8.6.889.

[42] Wendling F, Benquet P, Bartolomei F, and Jirsa V. Computational models of epileptiform activity. Journal of Neuroscience Methods 260 (2016), 233-251. DoI: 10 . 1016/ j.jneumeth. 2015.03.027.

[43] Hodgkin AL and Huxley AF. A quantitative description of membrane current and its application to conduction and excitation in nerve. The Journal of physiology 117.4 (1952), 500-544. DOI: 10.1113/jphysiol.1952. sp004764.

[44] Deco G, Jirsa VK, Robinson PA, Breakspear M, and Friston K. The dynamic brain: From spiking neurons to neural masses and cortical fields. PLoS Computational Biology 4.8 (2008). DoI: 10.1371/journal .pcbi . 1000092.

[45] Amari S. Dynamics of pattern formation in lateral-inhibition type neural fields. Biological cybernetics 27.2 (1977), 77-87. DOI: 10.1007/BF00337259.

[46] Coombes S. Waves, bumps, and patterns in neural field theories. Biological cybernetics 93.2 (2005), 91-108. DOI: $10.1007 / \mathrm{s} 00422-005-0574-y$.

[47] Jirsa VK, Stacey WC, Quilichini PP, Ivanov AI, and Bernard C. On the nature of seizure dynamics. Brain 137.8 (2014), 2210-2230. Dor: 10.1093/brain/awu133.

[48] Kalitzin SN, Velis DN, and Lopes da Silva FH. Stimulation-based anticipation and control of state transitions in the epileptic brain. Epilepsy and Behavior 17.3 (2010), 310-323. Dor: 10.1016/j . yebeh.2009.12.023.

[49] Benjamin O, Fitzgerald THB, Ashwin P, Tsaneva-Atanasova K, Chowdhury F, Richardson MP, and Terry JR. A phenomenological model of seizure initiation suggests network structure may explain seizure frequency in idiopathic generalised epilepsy. Journal of Mathematical Neuroscience 2.1 (2012), 1-41. DoI: 10 . 1186/2190-8567$2-1$.

[50] Lopes da Silva FH, Hoeks A, Smits H, and Zetterberg LH. Model of brain rhythmic activity. The alpha rhythm of the thalamus. Kybernetik 15.1 (1974), 27-37. Dor: 10. $1007 /$ BFQ0270757.

[51] Jansen BH, Zouridakis G, and Brandt ME. A neurophysiologically-based mathematical model of flash visual evoked potentials. Biological cybernetics 68.3 (1993), 275283. DOI: $10.1007 / B F 00224863$. 
[52] Jansen BH and Rit VG. Electroencephalogram and visual evoked potential generation in a mathematical model of coupled cortical columns. Biological cybernetics 73.4 (1995), 357-366. DOI: 10.1007/BFQ0199471.

[53] Wendling F, Bartolomei F, Bellanger JJ, and Chauvel P. Epileptic fast activity can be explained by a model of impaired GABAergic dendritic inhibition. European Journal of Neuroscience 15.9 (2002), 1499-1508. Dor: 10 . 1046/ j . 1460-9568 . 2002 . Q1985.x.

[54] David O and Friston KJ. A neural mass model for MEG/EEG: Coupling and neuronal dynamics. Neuroimage 20.3 (2003), 1743-1755. Dor: 10 . 1016/ j . neuroimage . 2003.07 .015 .

[55] Ursino M, Cona F, and Zavaglia M. The generation of rhythms within a cortical region: Analysis of a neural mass model. NeuroImage 52.3 (2010), 1080-1094. DoI: 10.1016/j .neuroimage . 2009. 12.084 .

[56] Wang P and Knösche TR. A Realistic Neural Mass Model of the Cortex with LaminarSpecific Connections and Synaptic Plasticity - Evaluation with Auditory Habituation. PLOS ONE 8.10 (Oct. 2013), 1-17. DoI: 10.1371/journal . pone. 0077876.

[57] Blenkinsop A, Valentin A, Richardson MP, and Terry JR. The dynamic evolution of focal-onset epilepsies - combining theoretical and clinical observations. European Journal of Neuroscience 36.2 (Feb. 2012), 2188-2200. DoI: 10 . 1111/ j . 1460 9568.2012.08082.x.

[58] David O, Harrison L, and Friston KJ. Modelling event-related responses in the brain. NeuroImage 25.3 (2005), 756-770. Dor: 10.1016/j . neuroimage .2004 . 12.030.

[59] Cona F, Zavaglia M, Massimini M, Rosanova M, and Ursino M. A neural mass model of interconnected regions simulates rhythm propagation observed via TMS-EEG. NeuroImage 57.3 (2011), 1045-1058. Dor: 10.1016/j . neuroimage .2011.05.007.

[60] Goodfellow M, Schindler K, and Baier G. Intermittent spike-wave dynamics in a heterogeneous, spatially extended neural mass model. NeuroImage 55.3 (2011), 920 932. Dor: $10.1016 / \mathrm{j}$. neuroimage . 2010.12.074.

[61] Ahmadizadeh S, Karoly PJ, Nešić D, Grayden DB, Cook MJ, Soudry D, and Freestone DR. Bifurcation analysis of two coupled Jansen-Rit neural mass models. PloS one 13.3 (2018), e0192842. Dor: 10.1371/journal . pone.0192842.

[62] Kameneva T, Ying T, Guo B, and Freestone DR. Neural mass models as a tool to investigate neural dynamics during seizures. Journal of computational neuroscience 42.2 (2017), 203-215. Dor: $10.1007 / \mathrm{s} 10827-017-0636-\mathrm{x}$.

[63] Karoly PJ, Kuhlmann L, Soudry D, Grayden DB, Cook MJ, and Freestone DR. Seizure pathways: A model-based investigation. PLoS computational biology 14.10 (2018), e1006403. Dor: 10.1371/journal . pcbi . 1006403.

[64] Goodfellow M, Schindler K, and Baier G. Self-organised transients in a neural mass model of epileptogenic tissue dynamics. NeuroImage 59.3 (2012), 2644-2660. Dor: 10.1016/j . neuroimage . 2011.08.060.

[65] Goodfellow M, Rummel C, Abela E, Richardson MP, Schindler K, and Terry JR. Estimation of brain network ictogenicity predicts outcome from epilepsy surgery. Scientific reports 6 (2016), 29215. DoI: 10.1038/srep29215. 
[66] Sinha N, Dauwels J, Kaiser M, Cash SS, Westover MB, Wang Y, and Taylor PN. Predicting neurosurgical outcomes in focal epilepsy patients using computational modelling. Brain 140.2 (2017), 319-332. Dor: 10.1093/brain/aww299.

[67] Jirsa VK, Proix T, Perdikis D, Woodman MM, Wang H, Gonzalez-Martinez J, Bernard C, Bènar C, Guye M, Chauvel P, and Bartolomei F. The Virtual Epileptic Patient: Individualized whole-brain models of epilepsy spread. NeuroImage 145.Part B (2017), 377-388. Dor: $10.1016 / \mathrm{j}$. neuroimage .2016.04.049.

[68] Lamberink HJ, Boshuisen K, Van Rijen PC, Gosselaar PH, and Braun KPJ. Changing profiles of pediatric epilepsy surgery candidates over time: A nationwide single-center experience from 1990 to 2011. Epilepsia 56.5 (2015), 717-725. Dor: 10.1111/epi . 12974.

[69] Spencer SS. Neural networks in human epilepsy: evidence of and implications for treatment. Epilepsia 43.3 (2002), 219-227. Dor: 10 . 1046/j . 1528-1157 . 2002 . 26901.x.

[70] Kramer MA and Cash SS. Epilepsy as a disorder of cortical network organization. The Neuroscientist 18.4 (2012), 360-372. DOI: 10.1177/1073858411422754.

[71] Van Diessen E, Zweiphenning WJEM, Jansen FE, Stam CJ, Braun KPJ, and Otte WM. Brain network organization in focal epilepsy: a systematic review and meta-analysis. PloS one 9.12 (2014), e114606. Dor: 10.1371/journal . pone. 0114606.

[72] Haneef $Z$ and Chiang S. Clinical correlates of graph theory findings in temporal lobe epilepsy. Seizure 23.10 (2014), 809-818. DoI: 10.1016/j . seizure .2014 .07.004.

[73] Chowdhury FA, Woldman W, FitzGerald THB, Elwes RDC, Nashef L, Terry JR, and Richardson MP. Revealing a brain network endophenotype in families with idiopathic generalised epilepsy. PloS one 9.10 (2014), e110136. Dor: 10. 1371 / journal . pone.0110136.

[74] Lüders H and Awad I. Epilepsy Surgery. Ed. by Lüders H. Raven Press, 1992. Chap. Conceptual Considerations, 51-62.

[75] Blümcke I, Thom M, Aronica E, Armstrong DD, Vinters HV, Palmini A, Jacques TS, Avanzini G, Barkovich AJ, Battaglia G, Becker A, Cepeda C, Cendes F, Colombo N, Crino P, Cross JH, Delalande O, Dubeau F, Duncan J, Guerrini R, Kahane P, Mathern G, Najm I, Özkara Ç, Raybaud C, Represa A, Roper SN, Salamon N, Schulze-Bonhage A, Tassi L, Vezzani A, and Spreafico R. The clinicopathologic spectrum of focal cortical dysplasias: A consensus classification proposed by an ad hoc task force of the ILAE Diagnostic Methods Commission 1. Epilepsia 52.1 (2011), 158-174. DoI: 10.1111/j.1528-1167.2010.02777.x.

[76] Terry JR, Benjamin O, and Richardson MP. Seizure generation: The role of nodes and networks. Epilepsia 53.9 (2012), e166-e169. DoI: 10.1111/j . 1528-1167. 2012. Q3560.x.

[77] McBain CJ, Boden P, and Hill RG. Rat hippocampal slices 'in vitro'display spontaneous epileptiform activity following long-term organotypic culture. Journal of neuroscience methods 27.1 (1989), 35-49. DoI: 10 .1016/0165-0270(89) 900514. 
[78] Liu J, Saponjian Y, Mahoney MM, Staley KJ, and Berdichevsky Y. Epileptogenesis in organotypic hippocampal cultures has limited dependence on culture medium composition. PloS one 12.2 (2017), e0172677. Dor: 10 . 1371/ journal . pone. Q172677.

[79] Suffczynski P, Lopes Da Silva FH, Parra J, Velis DN, Bouwman BM, Van Rijn CM, Van Hese P, Boon P, Khosravani H, Derchansky M, Carlen P, and Kalitzin S. Dynamics of epileptic phenomena determined from statistics of ictal transitions. IEEE Transactions on Biomedical Engineering 53.3 (2006), 524-532. DoI: 10. 1109/ TBME. 2005.869800.

[80] Park H.-J and Friston K. Structural and functional brain networks: from connections to cognition. Science 342.6158 (2013), 1238411. DoI: 10.1126/science. 1238411.

[81] Mill RD, Ito T, and Cole MW. From connectome to cognition: The search for mechanism in human functional brain networks. NeuroImage 160 (2017), 124-139. Dor: $10.1016 / \mathrm{j}$.neuroimage 2017.01 .060 .

[82] Tijms BM, Wink AM, De Haan W, Van der Flier WM, Stam CJ, Scheltens P, and Barkhof F. Alzheimer's disease: connecting findings from graph theoretical studies of brain networks. Neurobiology of aging 34.8 (2013), 2023-2036. DOI: 10 . 1016/j . neurobiolaging.2013.02.020.

[83] Engel Jr. J, Thompson PM, Stern JM, Staba RJ, Bragin A, and Mody I. Connectomics and epilepsy. Current opinion in neurology 26.2 (2013), 186-194. DoI: 10 . 1097 / WCO. ObQ13e32835ee5b8.

[84] Van Mierlo P, Papadopoulou M, Carrette E, Boon P, Vandenberghe S, Vonck K, and Marinazzo D. Functional brain connectivity from EEG in epilepsy: Seizure prediction and epileptogenic focus localization. Progress in neurobiology 121 (2014), 19-35. DoI: $10.1016 / \mathrm{j}$. pneurobio. 2014.06 .004 .

[85] Bartolomei F, Lagarde S, Wendling F, McGonigal A, Jirsa V, Guye M, and Bénar C. Defining epileptogenic networks: Contribution of SEEG and signal analysis. Epilepsia 58.7 (2017), 1131-1147. DoI: 10.1111/epi . 13791.

[86] Van den Heuvel MP and Fornito A. Brain networks in schizophrenia. Neuropsychology review 24.1 (2014), 32-48. DoI: 10.1007/s11065-014-9248-7.

[87] Khambhati AN, Davis KA, Lucas TH, Litt B, and Bassett DS. Virtual Cortical Resection Reveals Push-Pull Network Control Preceding Seizure Evolution. Neuron 91.5 (2016), 1170-1182. DoI: 10.1016/j . neuron. 2016.07 .039$.

[88] Rubinov M and Sporns O. Complex network measures of brain connectivity: Uses and interpretations. NeuroImage 52.3 (2010), 1059-1069. Dor: 10.1016/j . neuroimage . 2009.10 .003$.

[89] Wang HE, B'enar CG, Quilichini PP, Friston KJ, Jirsa VK, and Bernard C. A systematic framework for functional connectivity measures. Frontiers in Neuroscience 8.DEC (2014). DOI: 10.3389/fnins . 2014.00405.

[90] Friston KJ. Functional and effective connectivity: a review. Brain connectivity 1.1 (2011), 13-36. Dor: 10.1089/brain.2011.0008. 
[91] Keller CJ, Bickel S, Honey CJ, Groppe DM, Entz L, Craddock RC, Lado FA, Kelly C, Milham M, and Mehta AD. Neurophysiological investigation of spontaneous correlated and anticorrelated fluctuations of the BOLD signal. Journal of Neuroscience 33.15 (2013), 6333-6342. DoI: 10.1523/jneurosci . 4837-12.2013.

[92] Kramer MA, Eden UT, Cash SS, and Kolaczyk ED. Network inference with confidence from multivariate time series. Physical Review E 79.6 (2009), 061916. Dor: 10.1103/ PhysRevE.79.061916.

[93] Bressler SL and Seth AK. Wiener-Granger causality: a well established methodology. Neuroimage 58.2 (2011), 323-29. DoI: 10.1016/j . neuroimage. 2010.02.059.

[94] Pluim JPW, Maintz JBA, and Viergever MA. Mutual-information-based registration of medical images: A survey. IEEE Transactions on Medical Imaging 22.8 (2003), 986-1004. DoI: 10.1109/TMI . 2003.815867.

[95] Donos C, Mîndruţă I, Ciurea J, Mălîia MD, and Barborica A. A comparative study of the effects of pulse parameters for intracranial direct electrical stimulation in epilepsy. Clinical Neurophysiology 127.1 (2016), 91-101. DoI: 10 . 1016/j . clinph . 2015. Q2.013.

[96] Entz L, Tóth E, Keller CJ, Bickel S, Groppe DM, Fabó D, Kozák LR, Erőss L, Ulbert I, and Mehta AD. Evoked effective connectivity of the human neocortex. Human brain mapping 35.12 (2014), 5736-5753. DOI: 10.1002/hbm. 22581.

[97] Conner CR, Ellmore TM, DiSano MA, Pieters TA, Potter AW, and Tandon N. Anatomic and electro-physiologic connectivity of the language system: A combined DTI-CCEP study. Computers in biology and medicine 41.12 (2011), 1100-1109. DoI: 10.1016/j . compbiomed.2011.07.008.

[98] Donos C, Mălîia MD, Mîndruţă I, Popa I, Ene M, Bălănescu B, Ciurea A, and Barborica A. A connectomics approach combining structural and effective connectivity assessed by intracranial electrical stimulation. NeuroImage 132.Supplement C (2016), 344-358. Dor: 10.1016/j . neuroimage.2016.02.054.

[99] Parker CS, Clayden JD, Cardoso MJ, Rodionov R, Duncan JS, Scott C, Diehl B, and Ourselin S. Structural and effective connectivity in focal epilepsy. NeuroImage: Clinical 17 (2018), 943-952. Dor: 10.1016/j .nicl.2017.12.020.

[100] Barnett L and Seth AK. Behaviour of Granger causality under filtering: theoretical invariance and practical application. Journal of neuroscience methods 201.2 (2011), 404-419. DoI: 10.1016/j . jneumeth. 2011.08.010.

[101] Sinha N, Dauwels J, Wang Y, Cash SS, and Taylor PN. An in silico approach for presurgical evaluation of an epileptic cortex. 2014 36th Annual International Conference of the IEEE Engineering in Medicine and Biology Society. Aug. 2014, 4884-4887. DOI: 10.1109/EMBC.2014.6944718.

[102] Ding M, Chen Y, and Bressler SL. Granger causality: basic theory and application to neuroscience. Handbook of time series analysis: recent theoretical developments and applications. Ed. by Schelter B, Winterhalder M, and Timmer J. Wiley-VCH, 2006.

[103] Barnett L and Seth AK. The MVGC multivariate Granger causality toolbox: a new approach to Granger-causal inference. Journal of neuroscience methods 223 (2014), 50-68. DoI: $10.1016 / \mathrm{j}$. jneumeth. 2013.10 .018 . 
[104] Murin Y, Kim J, and Goldsmith A. Tracking epileptic seizure activity via information theoretic graphs. Signals, Systems and Computers, 2016 50th Asilomar Conference on. IEEE. 2016, 583-587. DoI: 10.1109/ACSSC. 2016.7869109.

[105] Murin Y, Kim J, Parvizi J, and Goldsmith A. SozRank: A new approach for localizing the epileptic seizure onset zone. PLoS computational biology 14.1 (2018), e1005953. DOI: 10.1371 journal.pcbi. 1005953.

[106] Seth AK. A MATLAB toolbox for Granger causal connectivity analysis. Journal of neuroscience methods 186.2 (2010), 262-273. DoI: 10 . 1016/j . jneumeth . 2009 . 11.020 .

[107] Rummel C, Abela E, Andrzejak RG, Hauf M, Pollo C, Müller M, Weisstanner C, Wiest R, and Schindler K. Resected brain tissue, seizure onset zone and quantitative EEG measures: Towards prediction of post-surgical seizure control. PLoS ONE 10.10 (2015). DoI: 10.1371/journal .pone.0141023.

[108] Rummel C, Abela E, Müller M, Hauf M, Scheidegger O, Wiest R, and Schindler $\mathrm{K}$. Uniform approach to linear and nonlinear interrelation patterns in multivariate time series. Physical Review E 83.6 (2011), 066215. DoI: 10.1103/PhysRevE . 83. 066215.

[109] Ponten SC, Daffertshofer A, Hillebrand A, and Stam CJ. The relationship between structural and functional connectivity: Graph theoretical analysis of an EEG neural mass model. NeuroImage 52.3 (2010), 985-994. Dor: 10.1016/ j . neuroimage . 2009.10 .049 .

[110] Jones SE, Beall EB, Najm I, Sakaie KE, Phillips MD, Zhang M, and GonzalezMartinez JA. Low consistency of four brain connectivity measures derived from intracranial electrode measurements. Frontiers in neurology 5 (2014). Dor: 10. 3389/ fneur. 2014.00272.

[111] Keller CJ, Bickel S, Entz L, Ulbert I, Milham MP, Kelly C, and Mehta AD. Intrinsic functional architecture predicts electrically evoked responses in the human brain. Proceedings of the National Academy of Sciences 108.25 (2011), 10308-10313. DoI: 10. 1073 /pnas. 1019750108 .

[112] Catani M, Jones DK, and ffytche DH. Perisylvian language networks of the human brain. Annals of Neurology 57.1 (2005), 8-16. DoI: 10.1002/ana . 20319.

[113] Matsumoto R, Nair DR, LaPresto E, Najm I, Bingaman W, Shibasaki H, and Lüders HO. Functional connectivity in the human language system: A cortico-cortical evoked potential study. Brain 127.10 (2004), 2316-2330. Dor: 10.1093/brain/awh246.

[114] Dronkers NF, Pinker S, and Damasio A. Language and the Aphasias. Principles of Neural Science. Ed. by Kandel ER, Schwartz JH, and Jessell TM. 4th edition. McGraw-Hill, 2000. Chap. 59, 1169-1187.

[115] Bastos AM and Schoffelen J.-M. A tutorial review of functional connectivity analysis methods and their interpretational pitfalls. Frontiers in Systems Neuroscience 9 (2016), 175. DOI: $10.3389 /$ fnsys . 2015.00175 .

[116] David O, Job AS, De Palma L, Hoffmann D, Minotti L, and Kahane P. Probabilistic functional tractography of the human cortex. NeuroImage 80 (2013), 307-317. DoI: 10.1016/j .neuroimage . 2013.05.075. 
[117] Shimada S, Kunii N, Kawai K, Matsuo T, Ishishita Y, Ibayashi K, and Saito N. Impact of volume-conducted potential in interpretation of cortico-cortical evoked potential: Detailed analysis of high-resolution electrocorticography using two mathematical approaches. Clinical Neurophysiology 128.4 (2017), 549-557. DOI: $10.1016 / \mathrm{j}$. clinph.2017.01.012.

[118] Kramer MA, Eden UT, Lepage KQ, Kolaczyk ED, Bianchi MT, and Cash SS. Emergence of persistent networks in long-term intracranial EEG recordings. Journal of Neuroscience 31.44 (2011), 15757-15767. DOI: 10.1523/JNEUROSCI . 2287-11.2011.

[119] Lopes MA, Richardson MP, Abela E, Rummel C, Schindler K, Goodfellow M, and Terry JR. An optimal strategy for epilepsy surgery: Disruption of the rich-club? PLOS Computational Biology 13.8 (Aug. 2017), 1-27. DoI: 10.1371/journal .pcbi . 1005637.

[120] Eissa TL and Schevon CA. The role of computational modelling in seizure localization. Brain 140.2 (2017), 254-256. DoI: 10. 1093/brain/aww332.

[121] Youngerman BE and McKhann II GM. From Nodes to Networks: Can Virtual Resections Predict Neurosurgical Outcomes in Focal Epilepsy? Neurosurgery 81.3 (2017), N25-N26. DoI: 10. 1093/neuros/nyx391.

[122] Noachtar S and Borggraefe I. Epilepsy surgery: A critical review. Epilepsy and Behavior 15.1 (2009), 66-72. Dor: 10.1016/j . yebeh .2009.02 .028.

[123] Jiménez-Jiménez D, Abete-Rivas M, Martín-López D, Lacruz ME, Selway RP, Valentín A, and Alarcón G. Incidence of functional bi-temporal connections in the human brain in vivo and their relevance to epilepsy surgery. Cortex 65 (2015), 208-218. DoI: 10.1016/j.cortex .2015.01.011.

[124] Wendling F, Bellanger JJ, Bartolomei F, and Chauvel P. Relevance of nonlinear lumped-parameter models in the analysis of depth-EEG epileptic signals. Biological cybernetics 83.4 (2000), 367-378. DOI: 10.1007/s004220000160.

[125] Sotero RC, Trujillo-Barreto NJ, Iturria-Medina Y, Carbonell F, and Jimenez JC. Realistically coupled neural mass models can generate EEG rhythms. Neural computation 19.2 (2007), 478-512. Dor: 10.1162/neco. 2007.19.2 . 478.

[126] Felleman DJ and Van Essen DC. Distributed hierarchical processing in the primate cerebral cortex. Cerebral Cortex 1.1 (1991), 1-47. Dor: 10.1093/cercor/1.1.1.

[127] Eissa TL, Dijkstra K, Brune C, Emerson RG, Van Putten MJAM, Goodman RR, McKhann GM, Schevon CA, Van Drongelen W, and Van Gils SA. Cross-scale effects of neural interactions during human neocortical seizure activity. Proceedings of the National Academy of Sciences of the United States of America 114.40 (2017), 1076110766. DOI: 10.1073/pnas . 1702490114.

[128] Boyer A, Duffau H, Vincent M, Ramdani S, Mandonnet E, Guiraud D, and Bonnetblanc F. Electrophysiological Activity Evoked by Direct Electrical Stimulation of the Human Brain: Interest of the P0 Component. 2018 40th Annual International Conference of the IEEE Engineering in Medicine and Biology Society (EMBC). July 2018, 2210-2213. DOI: 10.1109/EMBC.2018.8512733. 
[129] Wilson HR and Cowan JD. Excitatory and Inhibitory Interactions in Localized Populations of Model Neurons. Biophysical Journal 12.1 (1972), 1-24. Dor: 10 . 1016/ S0006-3495 (72) 86068-5.

[130] Molaee-Ardekani B, Benquet P, Bartolomei F, and Wendling F. Computational modeling of high-frequency oscillations at the onset of neocortical partial seizures: From 'altered structure' to 'dysfunction'. NeuroImage 52.3 (Sept. 2010), 1109-1122. Dor: $10.1016 / \mathrm{j}$. neuroimage . 2009.12.049.

[131] Touboul J, Wendling F, Chauvel P, and Faugeras O. Neural mass activity, bifurcations, and epilepsy. Neural computation 23.12 (2011), 3232-3286. DOI: 10. 1162 /NECO_ a_00206.

[132] David O, Bastin J, Chabardès S, Minotti L, and Kahane P. Studying Network Mechanisms Using Intracranial Stimulation in Epileptic Patients. Frontiers in Systems Neuroscience 4 (2010), 148. Dor: 10.3389/fnsys. 2010 . 00148.

[133] Dhooge A, Govaerts W, Kuznetsov YA, Meijer HGE, and Sautois B. New features of the software MatCont for bifurcation analysis of dynamical systems. Mathematical and Computer Modelling of Dynamical Systems 14.2 (2008), 147-175. DOI: 10 . $1080 / 13873950701742754$.

[134] Kuznetsov YA. Elements of Applied Bifurcation Theory. 3th. Springer, 2004.

[135] Chehelcheraghi M, Nakatani C, Steur E, and Van Leeuwen C. A neural mass model of phase-amplitude coupling. Biological Cybernetics 110.2-3 (June 2016), 171-192. DOI: $10.1007 / \mathrm{s} 00422-016-0687-5$.

[136] Traub RD, Whittington MA, Buhl EH, LeBeau FEN, Bibbig A, Boyd S, Cross H, and Baldeweg T. A Possible Role for Gap Junctions in Generation of Very Fast EEG Oscillations Preceding the Onset of, and Perhaps Initiating, Seizures. Epilepsia 42.2 (2001), 153-170. DOI: 10.1046/j . 1528-1157.2001.26900.x.

[137] Goodfellow M. Spatio-temporal modelling and analysis of epileptiform EEG. PhD thesis. University of Manchester, 2011. URL: https : / / core . ac . uk/download/ pdf/40015029.pdf.

[138] Babajani-Feremi A and Soltanian-Zadeh H. Multi-area neural mass modeling of EEG and MEG signals. NeuroImage 52.3 (2010), 793-811. DoI: 10 . $1016 / \mathrm{j}$. neuroimage.2010.01.034.

[139] Spiegler A, Kiebel SJ, Atay FM, and Knösche TR. Bifurcation analysis of neural mass models: Impact of extrinsic inputs and dendritic time constants. NeuroImage 52.3 (2010), 1041-1058. DoI: 10.1016/j .neuroimage .2009.12.081.

[140] Shamas M, Benquet P, Merlet I, Khalil M, El Falou W, Nica A, and Wendling F. On the origin of epileptic High Frequency Oscillations observed on clinical electrodes. Clinical Neurophysiology 129.4 (2018), 829-841. Dor: 10.1016/j . clinph. 2018. 01.062 .

[141] Alarcón G, Martinez J, Kerai SV, Lacruz ME, Quiroga RQ, Selway RP, Richardson MP, Seoane JJG, and Valentín A. In vivo neuronal firing patterns during human epileptiform discharges replicated by electrical stimulation. Clinical Neurophysiology 123.9 (2012), 1736-1744. DoI: 10.1016/j .clinph.2012.02.062. 
[142] Nayak D, Valentín A, Selway RP, and Alarcón G. Can single pulse electrical stimulation provoke responses similar to spontaneous interictal epileptiform discharges? Clinical Neurophysiology 125.7 (2014), 1306-1311. Dor: 10 . 1016/ j . clinph . 2013.11.019.

[143] Homburg AJ and Sandstede B. Homoclinic and heteroclinic bifurcations in vector fields. Vol. 3. Handbook of Dynamical Systems C. 2010, 379-524.

[144] Van der Kamp W, Zwinderman AH, Ferrari MD, and Van Dijk JG. Cortical excitability and response variability of transcranial magnetic stimulation. Journal of clinical neurophysiology 13.2 (1996), 164-171. DOI: 10 . 1097 /00004691-199603000 00007.

[145] Ridding MC and Rothwell JC. Stimulus/response curves as a method of measuring motor cortical excitability in man. Electroencephalography and Clinical Neurophysiology/Electromyography and Motor Control 105.5 (1997), 340-344. DoI: 10 . 1016/ S0924-980X (97)00041-6.

[146] Boulogne S, Andre-Obadia N, Kimiskidis VK, Ryvlin P, and Rheims S. Corticocortical and motor evoked potentials to single and paired-pulse stimuli: An exploratory transcranial magnetic and intracranial electric brain stimulation study. Human Brain Mapping 37.11 (2016), 3767-3778. DoI: 10.1002/hbm. 23274.

[147] Izhikevich EM. Neural excitability, spiking and bursting. International journal of bifurcation and chaos 10.06 (2000), 1171-1266. DOI: 10.1142/S0218127400000840.

[148] Best J, Park C, Terman D, and Wilson C. Transitions between irregular and rhythmic firing patterns in excitatory-inhibitory neuronal networks. Journal of computational neuroscience 23.2 (2007), 217-235. DOI: 10.1007/s10827-007-0029-7.

[149] Teka W, Tabak J, Vo T, Wechselberger M, and Bertram R. The dynamics underlying pseudo-plateau bursting in a pituitary cell model. The Journal of Mathematical Neuroscience 1.1 (2011), 12. DoI: 10.1186/2190-8567-1-12.

[150] Desroches M, Faugeras O, and Krupa M. Slow-Fast Transitions to Seizure States in the Wendling-Chauvel Neural Mass Model. Opera Medica et Physiologica 2.3 (Dec. 2016), 228-234. DOI: 10.20388/omp2016.003.0038.

[151] Fenichel N and Moser JK. Persistence and smoothness of invariant manifolds for flows. Indiana University Mathematics Journal 21.3 (1971), 193-226. DoI: 10. 1512/iumj . 1971.21 .21017$.

[152] Fenichel N. Geometric singular perturbation theory for ordinary differential equations. Journal of differential equations 31.1 (1979), 53-98. Dor: 10 . 1016/0022-0396 (79) 90152-9.

[153] Krupa M and Szmolyan P. Extending geometric singular perturbation theory to nonhyperbolic points-fold and canard points in two dimensions. SIAM journal on mathematical analysis 33.2 (2001), 286-314. Dor: 10.1137/S0036141099360919.

[154] Krupa M and Wechselberger M. Local analysis near a folded saddle-node singularity. Journal of Differential Equations 248.12 (2010), 2841-2888. Dor: 10. 1016/j . jde. 2010.02 .006 . 
[155] Farjami S, Kirk V, and Osinga H. Computing the Stable Manifold of a Saddle Slow Manifold. SIAM Journal on Applied Dynamical Systems 17.1 (2018), 350-379. Dor: 10.1137/17M1132458.

[156] Grimbert F and Faugeras O. Bifurcation analysis of Jansen's neural mass model. Neural computation 18.12 (2006), 3052-3068. Dor: 10 . 1162/neco . 2006 . 18 . 12 . 3052 .

[157] David O, Woźniak A, Minotti L, and Kahane P. Preictal short-term plasticity induced by intracerebral $1 \mathrm{~Hz}$ stimulation. Neuroimage 39.4 (2008), 1633-1646. DoI: 10 . 1016/j .neuroimage . 2007.11.005.

[158] Van Blooijs D, Huiskamp G, Hebbink GJ, Van 't Klooster M, Meijer HGE, Van Gils SA, Gosselaar P, and Leijten F. The spatial relation between early and delayed responses evoked by single pulse electrical stimulation in pre-surgical evaluation of epilepsy patients. 31st International Epilepsy Congress Istanbul, Turkey 5th-9th September, 2015. 2015, 148-148. Dor: 10.1111/epi . 13241.

[159] Enatsu R, Piao Z, O’Connor T, Horning K, Mosher J, Burgess R, Bingaman W, and Nair D. Cortical excitability varies upon ictal onset patterns in neocortical epilepsy: a cortico-cortical evoked potential study. Clinical Neurophysiology 123.2 (2012), 252260. DoI: $10.1016 /$ j.clinph. 2011.06 .030 .

[160] Kobayashi K, Matsumoto R, Matsuhashi M, Usami K, Shimotake A, Kunieda T, Kikuchi T, Yoshida K, Mikuni N, Miyamoto S, Fukuyama H, Takahashi R, and Ikeda A. High frequency activity overriding cortico-cortical evoked potentials reflects altered excitability in the human epileptic focus. Clinical Neurophysiology 128.9 (2017), 1673-1681. DoI: 10.1016/j .clinph.2017.06.249.

[161] Van Blooijs D, Hebbink GJ, Leijten FSS, Meijer HGE, Van Gils SA, and Huiskamp GJM. An improved protocol for single pulse electrical stimulation for identifying epileptogenic tissue in epilepsy surgery patients. Brain Stimulation: Basic, Translational, and Clinical Research in Neuromodulation 10.2 (2017), 529. DOI: 10.1016/ j.brs.2017.01.541.

[162] Van Blooijs D. Improving the SPES protocol by automating ER and DR detection and evaluation of the spatial relation between ERs and DRs. MA thesis. University of Twente, 2015. uRL: http://purl . utwente.nl/essays/68704.

[163] Tijssen M. Single Pulse Electrical Stimulation - Automatic detection of delayed responses. MA thesis. University of Twente, 2016.

[164] d'Angremont E, Huiskamp GJ, Leijten FS, Brune C, and Van Putten MJ. Machine learning for the analysis of single pulse stimula-tion in electrocorticography. Clinical Neurophysiology 129.Suppl. 1 (2018), e125. Dor: 10.1016/j . clinph. 2018. 04 . 317.

[165] d'Angremont E. Towards an automated analysis for the delineation of focal epilepsy: single pulse electrical stimulation and machine learning. MA thesis. University of Twente, 2018. uRL: http://purl . utwente.nl/essays/74818.

[166] Zijlmans M, Jiruska P, Zelmann R, Leijten FSS, Jefferys JGR, and Gotman J. Highfrequency oscillations as a new biomarker in epilepsy. Annals of neurology 71.2 (2012), 169-178. DoI: 10.1002/ana.22548. 
[167] Jayakar P, Gotman J, Harvey AS, Palmini A, Tassi L, Schomer D, Dubeau F, Bartolomei F, Yu A, Kršek P, Velis D, and Kahane P. Diagnostic utility of invasive EEG for epilepsy surgery: indications, modalities, and techniques. Epilepsia 57.11 (2016), 1735-1747. DOI: 10.1111/epi. 13515.

[168] Van 't Klooster MA, Leijten FSS, Huiskamp G, Ronner HE, Baayen JC, Van Rijen PC, Eijkemans MJC, Braun KPJ, and Zijlmans M. High frequency oscillations in the intra-operative ECoG to guide epilepsy surgery ("The HFO Trial"): study protocol for a randomized controlled trial. Trials 16.1 (2015), 422. Dor: 10.1186/s13063015-0932-6.

[169] Roessler K, Heynold E, Buchfelder M, Stefan H, and Hamer HM. Current value of intraoperative electrocorticography (iopECoG). Epilepsy \& Behavior 91 (2019), 2024. DoI: $10.1016 / \mathrm{j}$. yebeh.2018.06.053.

[170] Yamao Y, Matsumoto R, Kunieda T, Arakawa Y, Kobayashi K, Usami K, Shibata S, Kikuchi T, Sawamoto N, Mikuni N, Ikeda A, Fukuyama H, and Miyamoto S. Intraoperative dorsal language network mapping by using single-pulse electrical stimulation. Human brain mapping 35.9 (2014), 4345-4361. DOI: 10.1002/hbm. 22479.

[171] Yamao Y, Suzuki K, Kunieda T, Matsumoto R, Arakawa Y, Nakae T, Nishida S, Inano R, Shibata S, Shimotake A, Kikuchi T, Sawamoto N, Mikuni N, Ikeda A, Fukuyama H, and Miyamoto S. Clinical impact of intraoperative CCEP monitoring in evaluating the dorsal language white matter pathway. Human brain mapping 38.4 (2017), 1977-1991. DOI: 10. 1002/hbm. 23498.

[172] Hindriks R and Van Putten MJAM. Meanfield modeling of propofol-induced changes in spontaneous EEG rhythms. NeuroImage 60.4 (2012), 2323-2334. DOI: 10.1016/ j . neuroimage.2012.02.042.

[173] Van Diessen E, Hanemaaijer JI, Otte WM, Zelmann R, Jacobs J, Jansen FE, Dubeau F, Stam CJ, Gotman J, and Zijlmans M. Are high frequency oscillations associated with altered network topology in partial epilepsy? NeuroImage 82 (2013), 564-573. Dor: $10.1016 / \mathrm{j}$.neuroimage 2013.06 .031 .

[174] Ponten SC, Bartolomei F, and Stam CJ. Small-world networks and epilepsy: graph theoretical analysis of intracerebrally recorded mesial temporal lobe seizures. Clinical neurophysiology 118.4 (2007), 918-927. DOI: 10.1016/j . clinph.2006.12.002.

[175] Schindler KA, Bialonski S, Horstmann M.-T, Elger CE, and Lehnertz K. Evolving functional network properties and synchronizability during human epileptic seizures. Chaos: An Interdisciplinary Journal of Nonlinear Science 18.3 (2008), 033119. DoI: 10.1063/1.2966112.

[176] Kramer MA, Kolaczyk ED, and Kirsch HE. Emergent network topology at seizure onset in humans. Epilepsy research 79.2-3 (2008), 173-186. DoI: 10 . $1016 / \mathrm{j}$. eplepsyres.2008.02.002.

[177] Khambhati AN, Davis KA, Oommen BS, Chen SH, Lucas TH, Litt B, and Bassett DS. Dynamic network drivers of seizure generation, propagation and termination in human neocortical epilepsy. PLoS computational biology 11.12 (2015), e1004608. DoI: 10.1371 /journal.pcbi. 1004608 . 
[178] Wilke C, Van Drongelen W, Kohrman M, and He B. Neocortical seizure foci localization by means of a directed transfer function method. Epilepsia 51.4 (2010), 564-572. DOI: $10.1111 / \mathrm{j} .1528-1167.2009 .02329 . \mathrm{x}$.

[179] Wilke C, Worrell G, and He B. Graph analysis of epileptogenic networks in human partial epilepsy. Epilepsia 52.1 (2011), 84-93. Dor: 10.1111/j .1528-1167.2010. Q2785.x.

[180] Van Mierlo P, Carrette E, Hallez H, Raedt R, Meurs A, Vandenberghe S, Van Roost D, Boon P, Staelens S, and Vonck K. Ictal-onset localization through connectivity analysis of intracranial EEG signals in patients with refractory epilepsy. Epilepsia 54.8 (2013), 1409-1418. DoI: 10.1111/epi . 12206.

[181] Park E.-H and Madsen JR. Granger causality analysis of interictal iEEG predicts seizure focus and ultimate resection. Neurosurgery 82.1 (2017), 99-109. DOI: 10 . 1093/neuros/nyx195.

[182] Lopes MA, Richardson MP, Abela E, Rummel C, Schindler K, Goodfellow M, and Terry JR. Elevated ictal brain network ictogenicity enables prediction of optimal seizure control. Frontiers in neurology 9 (2018), 98. DoI: 10.3389/fneur . 2018. 00098.

[183] Van Diessen E, Numan T, Van Dellen E, Van Der Kooi AW, Boersma M, Hofman D, Van Lutterveld R, Van Dijk BW, Van Straaten ECW, Hillebrand A, and Stam CJ. Opportunities and methodological challenges in EEG and MEG resting state functional brain network research. Clinical Neurophysiology 126.8 (2015), 14681481. DOI: $10.1016 / \mathrm{j}$. clinph.2014.11.018.

[184] Fraschini M, Demuru M, Crobe A, Marrosu F, Stam CJ, and Hillebrand A. The effect of epoch length on estimated EEG functional connectivity and brain network organisation. Journal of neural engineering 13.3 (2016), 036015. Dor: 10 . 1088 / $1741-2560 / 13 / 3 / 036015$.

[185] Hutchings F, Han CE, Keller SS, Weber B, Taylor PN, and Kaiser M. Predicting surgery targets in temporal lobe epilepsy through structural connectome based simulations. PLoS computational biology 11.12 (2015), e1004642. DoI: 10 . 1371/journal . pcbi. 1004642 .

[186] Proix T, Bartolomei F, Guye M, and Jirsa VK. Individual brain structure and modelling predict seizure propagation. Brain 140.3 (2017), 641-654. DoI: 10 . 1093/brain/ awx004.

[187] Van 't Klooster MA, Van Klink NEC, Van Blooijs D, Ferrier CH, Braun KPJ, Leijten FSS, Huiskamp GJM, and Zijlmans M. Evoked versus spontaneous high frequency oscillations in the chronic electrocorticogram in focal epilepsy. Clinical Neurophysiology 128.5 (2017), 858-866.

[188] Junges L, Lopes MA, Terry JR, and Goodfellow M. The role that choice of model plays in predictions for epilepsy surgery. Scientific reports 9.1 (2019), 7351. DoI: 10. 1038/s41598-019-43871-7. 


\section{Summary}

Epilepsy is a common neurological disease, affecting around $1 \%$ of the world population. Epilepsy is characterized by the occurrence of transient periods of abnormal excessive or synchronous neuronal activity, called epileptic seizures. Epilepsy surgery may provide a cure for patients with focal epilepsy and aims at removing the epileptogenic zone. Accurate delineation of the epileptogenic zone, however, remains one of the challenges in epilepsy surgery.

Traditionally, epilepsy surgery mainly focuses on removing pathological cortex in the anatomic sense, while recent developments suggests that epilepsy is also a brain network disease. Computational models for epilepsy offer a framework to study the joint effect of local, intrinsic epileptogenicity and network interaction and allow to incorporate patientspecific information. In epilepsy patients who undergo long-term invasive EEG recordings prior to surgery, this information might be obtained using single pulse electrical stimulation (SPES). Using brief electrical pulses SPES evokes early responses, representing connectivity, and delayed responses which are a biomarker for epileptogenic cortex. In this thesis the added value of combining computational network models and SPES for epilepsy surgery is investigated.

First, we study the effects of surgery on the seizure rate in a simple network model consisting of four interconnected neuronal populations. One of these populations can be configured to be hyperexcitable, modelling a pathological region of cortex. Model simulations show that removal of normal populations located at a crucial spot in the network, is typically more effective in reducing seizure rate than removing the hyperexcitable node. This result strengthens the idea that besides localizing pathological tissue also network structure must be taken into account for successful epilepsy surgery.

Second, we compare connectivity probed using SPES with two traditional methods, i.e. cross-correlation and Granger causality, that infer connectivity from ongoing brain activity recorded using intracranial EEG. All three methods yield primarily nearest neighbour connections, however SPES networks are usually connected more densely and include more distant connections than cross-correlation and Granger causality networks. We find that strong connections in the cross-correlation network form more or less a subset of the SPES network, while Granger causality and SPES networks are related more weakly. Connectivity known to exist between Broca's and Wernicke's area, the two major hubs in the language circuit, is only found in SPES networks. An explanation for the differences between the networks might be that cross-correlation and Granger causality infer connectivity from passive observations, where SPES probes connectivity actively.

Next, we use a data-driven modelling approach to study the mechanism generating delayed responses evoked during SPES. Using data of 11 patients we confirm our hypothesis that delayed responses are indirect responses triggered by early response activity. We show using two feedforward coupled neural mass models that delayed responses can be generated when input to a neural mass falls below a threshold, forcing it into a spiking regime temporarily. The combination of the threshold with noisy background input explains the typical stochastic appearance of delayed responses. The probability for a delayed response to occur, depends on both the intrinsic excitability of a neural mass and the strength of its input. These results gives a theoretical basis for the use of delayed responses as a biomarker for the epileptogenic zone, confirming earlier clinical observations. 
The mechanism we propose to model delayed responses is an example of a large non-linear response to a short transient input which appears abruptly while increasing the stimulation strength in an excitable system. Using slow-fast analysis we study the transition from small, more or less linear to large, non-linear responses in more detail in our model for delayed responses. We find that the two response types are separated by a high-dimensional stable manifold of a saddle. Large pathological responses appear if the fast subsystem escapes from this manifold to another attractor. The orbit of the critical response can be formulated as a boundary value problem with one free parameter and can be used to study the dependency of the transition between the two response types upon the system parameters.

Currently, the usage of SPES to assist with localization of the epileptogenic zone is limited to patients undergoing long-term intracranial EEG monitoring. The SPES protocol might be accelerated by exploiting the relation between early and delayed responses. Analysis of delayed responses might be sped-up and improved by using automatic detection based on machine learning. Ultimately, intraoperative use of SPES for precise localization of the epileptogenic zone might be feasible, although still many efforts need to be made.

Computational network models offer an interesting framework to explore effects of epilepsy surgery. Multiple studies have shown that such models in combination with patientspecific networks can predict the outcome of surgery. Networks derived from SPES might be an interesting option to use as these networks incorporate more physiological long-range connections than functional networks derived from ongoing intracranial EEG recordings. An important next step to improve computational network models for epilepsy surgery is to incorporate patient-specific information about local excitability. Our results suggest that delayed responses evoked during SPES might be a good candidate marker for this, with the advantage over other biomarkers that it can be probed actively. In conclusion, SPES and patient-specific computational network models form a promising combination that have great potential to improve epilepsy surgery. 


\section{Samenvatting}

Epilepsie is een veel voorkomende neurologische aandoening waar ongeveer $1 \%$ van de wereld bevolking aan lijdt. Epilepsie wordt gekenmerkt door het voorkomen van perioden met excessieve of abnormaal synchrone hersenactiviteit, die epileptische aanvallen worden genoemd. Epilepsiechirurgie kan een genezende behandeling zijn voor patiënten met focale epilepsie en heeft als doel de epileptogene zone te verwijderen. Het nauwkeurig afbakenen van de epileptogene zone is een van de uitdagingen op het gebied van epilepsiechirurgie.

Traditioneel gezien richt epilepsiechirurgie zich op het verwijderen van structureel pathologisch hersenweefsel. Aan de andere kant suggereren recente ontwikkeling dat epilepsie mede een aandoening van het hersennetwerk is. Computationele modellen voor epilepsie bieden een structuur om de gezamenlijke invloed van lokale, intrinsieke epileptogeniciteit en netwerkinteracties te onderzoeken en geven de mogelijkheid om hierin patiëntspecifieke informatie op te nemen. Voor epilepsiepatiënten die voor hun operatie een langdurige invasieve EEG-registratie ondergaan kan deze informatie mogelijk verkregen worden door enkele puls elektrische stimulatie (Engels: single pulse electrical stimulation, SPES). Door middel van korte elektrische pulsen lokt SPES vroege en late corticale responsen uit. Waar vroege responsen een gevolg zijn van connectiviteit, zijn late responsen een biomarker voor epileptogene cortex. In dit proefschrift wordt de toegevoegde waarde van de combinatie van computationele netwerkmodellen en SPES onderzocht.

Allereerst onderzoeken we het effect van chirurgie op de aanvalsfrequentie in een eenvoudig netwerkmodel bestaande uit vier met elkaar verbonden neuronale populaties. Een van deze populaties kan hyperexciteerbaar gemaakt worden om een pathologisch stukje cortex na te bootsen. Modelsimulaties laten zien dat het verwijderen van een normale populatie die zich op een strategische plaats in het netwerk bevindt meestal effectiever is om de aanvalsfrequentie te reduceren dan het weghalen van de hyperexciteerbare populatie. Dit resultaat versterkt het idee dat voor succesvolle epilepsiechirurgie naast het pathologisch hersenweefsel ook rekening gehouden moet worden met de netwerkstructuur.

Als tweede vergelijken we netwerken bepaald met SPES met twee traditionele methoden, i.e. kruiscorrelatie en Granger-causaliteit. Deze beide methoden leiden connectiviteit af uit spontane hersenactiviteit die gemeten is met intracranieel EEG. Alle drie methoden vinden hoofdzakelijk verbindingen tussen dichtstbijzijnde buren, hoewel SPES-netwerken in de regel dichter verbonden zijn en meer langeafstandsverbindingen bevatten dan kruiscorrelatie en Granger-causaliteit netwerken. De sterke verbindingen uit de kruiscorrelatienetwerken vormen min of meer een strikte deelverzameling van de SPES-verbindingen, terwijl Granger causaliteit en SPES-netwerken zwakker aan elkaar gerelateerd zijn. Bekende verbindingen tussen de gebieden van Broca en Wernicke, twee knooppunten in het taalnetwerk, worden alleen in het SPES-netwerk gevonden. Een mogelijke verklaring voor de verschillen tussen de netwerken is dat kruiscorrelatie en Granger-causaliteit verbindingen bepalen uit passieve observaties, terwijl SPES actief de connectiviteit bepaalt.

Vervolgens gebruiken we een datagedreven aanpak om het mechanisme onderliggend aan late SPES-responsen te bestuderen. Gebruikmakend van de data van 11 patiënten bevestigen we onze hypothese dat late SPES-responsen indirecte responsen zijn die worden veroorzaakt door de activiteit van vroege responsen. We laten met behulp van twee voorwaartsgekoppelde neuralemassamodellen zien dat late responsen gegenereerd kunnen worden wanneer de invoer tot een neurale massa onder een zekere drempel komt. De neurale massa komt daardoor 
tijdelijk in een toestand waarin deze epileptische pieken vertoont. De combinatie van deze drempel met fluctuerende achtergrondinvoer tot de neurale massa verklaart het kenmerkende stochastische voorkomen van late responsen. Deze resultaten geven een theoretische onderbouwing voor het gebruik van late responsen als biomarker voor de epileptogene zone en bevestigen eerdere klinische waarnemingen.

Het mechanisme dat wij gebruiken om een late SPES-respons te modelleren is een voorbeeld van een grote, niet-lineaire reactie op een korte, tijdelijke invoer, die abrupt verschijnt als de stimulatiesterkte in een exciteerbaar systeem wordt verhoogd. Gebruikmakend van een snel-langzaamanalyse onderzoeken we de transitie van kleine, min of meer lineaire naar grote, niet-lineaire responsen in meer detail in ons model voor late SPES-responsen. De twee verschillende responstypen worden gescheiden door een hoogdimensionale variëteit van een zadelpunt. Grote, pathologische responsen ontstaan als het snelle deelsysteem ontsnapt van deze variëteit naar een andere aantrekker. De baan van de kritische respons kan worden geformuleerd als een randwaardenprobleem met een vrije parameter en kan worden gebruikt om de invloed van de systeemparameters op de transitie tussen de twee respons typen te onderzoeken.

Het gebruik van SPES om te ondersteunen met de lokalisatie van de epileptogene zone is op dit moment gelimiteerd tot patiënten die langdurig intracraniële EEG-onderzoek ondergaan. Een mogelijkheid om het SPES-protocol te versnellen is door gebruik te maken van de relatie tussen vroege en late responsen. De analyse van de late responsen kan worden versneld en verbeterd door automatische detectie gebaseerd op machinaal leren. Uiteindelijk is het misschien mogelijk om SPES intraoperatief toe te passen om de epileptogene zone te bepalen, hoewel dit nog de nodige inspanning zal vergen.

Computationele netwerkmodellen bieden een interessante structuur om de effecten van epilepsiechirurgie te verkennen. Meerdere studies hebben aangetoond dat zulke modellen in combinatie met patiëntspecifieke netwerken het resultaat van chirurgie kunnen voorspellen. Voor deze toepassing lijkt het interessant om ook SPES-netwerken te gebruiken, aangezien deze meer fysiologische langeafstandsverbindingen bevatten dan functionele netwerken afgeleid uit spontane intracraniële EEG-metingen. Een belangrijke vervolgstap om computationele netwerkmodellen voor epilepsiechirurgie te verbeteren is om patiëntspecifieke informatie over lokale exciteerbaarheid te includeren. Onze resultaten suggereren dat late SPES-responsen hiervoor een goede kandidaat zijn, met als voordeel ten opzichte van andere biomarkers dat ze op een actieve manier uit te lokken zijn. Concluderend kan gesteld worden dat SPES en patiëntspecifieke, computationele netwerkmodellen een veelbelovende combinatie vormen met veel potentieel om epilepsiechirurgie te verbeteren. 


\section{Dankwoord}

Na bijna vijf jaar is het dan zover, mijn proefschrift is klaar en mijn promotie nadert nu echt het eind. Ik kijk met genoegen terug op deze tijd. Graag wil ik een ieder bedanken die op enige manier betrokken was bij mijn proefschrift waarbij een aantal mensen in het bijzonder.

Beste Stephan. Als promotor hield jij de grote lijnen van het proces in de gaten. Het was handig om iemand te hebben die het overzicht hield en af en toe een knoop doorhakte. Daarnaast had je ook interesse voor de wiskundige details van mijn werk, waarbij je vaak goede suggesties aandroeg om nader te onderzoeken.

Beste Frans. Ik denk niet dat er veel neurologen zijn die copromotor zijn van een toegepast wiskundige. Voor mij geeft dit aan hoe jij openstaat voor multidisciplinair onderzoek om zo epilepsie nog beter te kunnen behandelen. Jouw klinische blik heeft zeker een meerwaarde gehad voor mijn proefschrift, in het bijzonder bij het publiceren van het tweede hoofdstuk.

Beste Hil. De afgelopen jaren was jij mijn dagelijks begeleider en hebben we nauw samengewerkt. Na mij ben jij zonder twijfel degene die het meeste tijd in dit proefschrift heeft zitten. Ik waardeer het dat je altijd probeerde tijd vrij te maken, ook als je die eigenlijk niet had of soms zomaar even langsliep voor een praatje. Je me heel veel geleerd, niet alleen op wiskundig gebied, maar juist ook bij alles wat erom heen komt.

Beste Geertjan. Helaas is het niet mogelijk om drie copromotoren te hebben, want dat was eigenlijk wel terecht geweest. Jij was voor veel dingen mijn eerste aanspreekpunt op het UMC en kent als geen ander alle aspecten van de SPES-data. Ook waardeer ik de interessante gesprekken die we hadden tijdens mijn wekelijkse dag op het UMC.

I want to thank all members of my graduation committee for their time and effort to read my thesis.

Mijn dank gaat ook uit naar ZonMW en het Nederlands epilepsiefonds die het onderzoeksproject 'From computational models of epilepsy to clinical protocols' en daarmee mijn promotie financieel mogelijk hebben gemaakt.

Dorien, Marij, Emile en Philippa die ieder op hun eigen manier als student betrokken waren bij dit project wil ik bedanken voor hun inzet. In het bijzonder gaat mijn dank uit naar Dorien, die later ook nog een jaar als onderzoeker deel uitmaakte van het project en de drijvende kracht was achter de klinische studie naar SPES.

To all colleagues from the applied analyses group of the UT: Stephan, Hil, Christoph, Huan, Bettina, Koen, Leonie, Yoeri, Manu and Len, I enjoyed the interesting and open discussions during the group meetings. Tracy thank you for proofreading the third chapter, which definitely helped to get this work published. Mariëlle en Linda bedankt voor het regelen van afspraken en andere dingen die soms zo van zelfsprekend lijken te gebeuren.

Mijn collega's van de KNF in het UMC: Frans, Geertjan, Cyrille, Tineke, Maieke, Sandra, Nico, Maryse, Nicole, Dorien, Willemiek, Anne, Brian, Lotte en Matteo en de vele studenten wil ik bedanken voor de goede sfeer en de gezellige lunchpauzes. Ondanks mijn niet-klinische achtergrond heb ik me er altijd welkom gevoeld.

Mathijs, Mathijs, Coen, Roeland en Thom, onze kolo.i.d. middagen en avonden zijn altijd gezellig. Opdat er nog velen mogen volgen.

Rutger, Mariët en Jonne, bedankt voor jullie luisterend oor, goede adviezen en gezelligheid.

Tot slot de twee belangrijkste mensen voor mij, mijn ouders, Henk en Ineke op wie ik altijd terug kan vallen. Bedankt voor jullie enorme steun, zonder jullie was me dit zeker niet gelukt. 


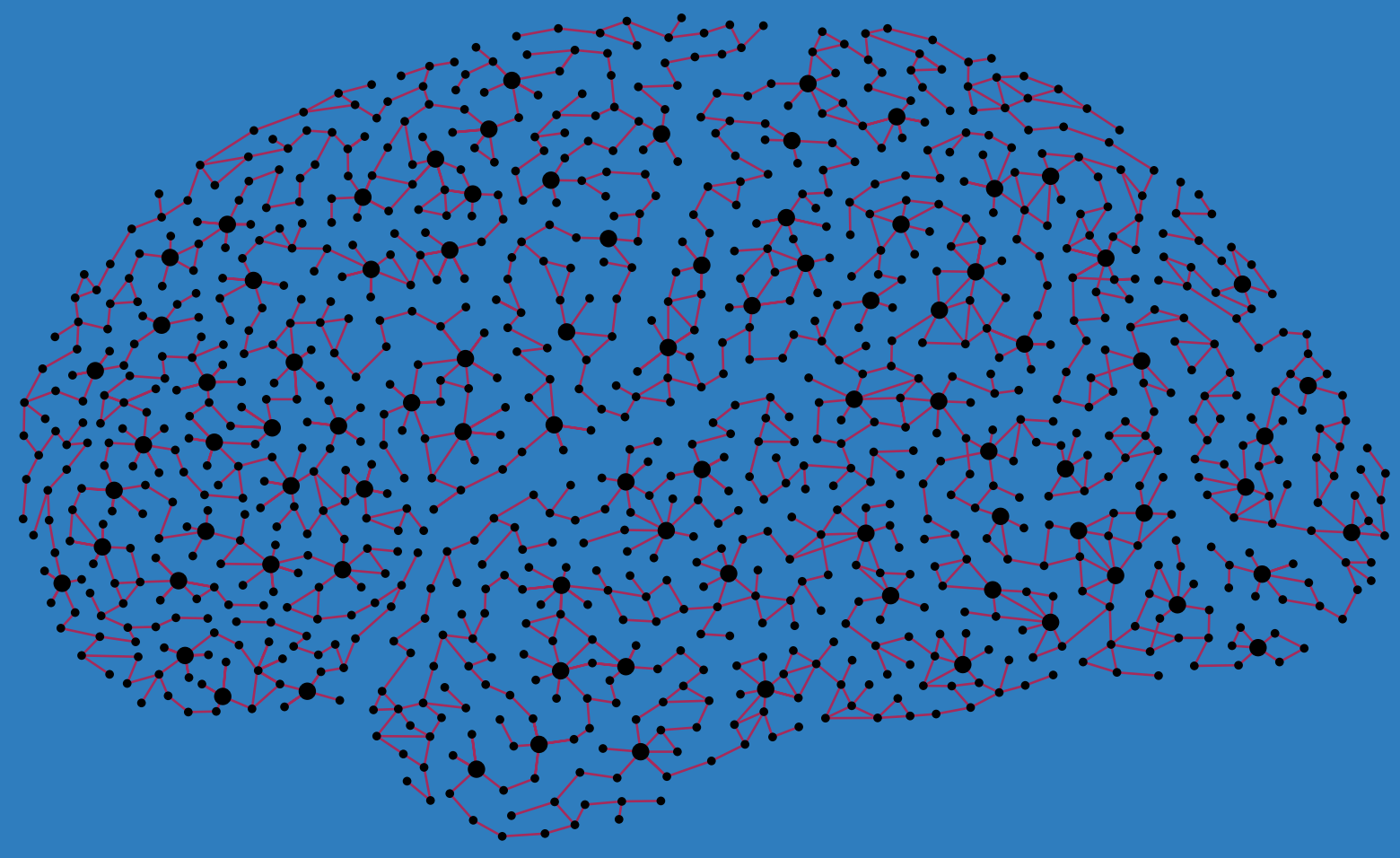

